\title{
TOROIDAL VARIETIES AND THE WEAK FACTORIZATION THEOREM
}

\author{
JAROSŁAW WŁODARCZYK
}

\begin{abstract}
We develop the theory of stratified toroidal varieties, which gives, together with the theory of birational cobordisms 73 , a proof of the weak factorization conjecture for birational maps in characteristic zero: a birational map between complete nonsingular varieties over an algebraically closed field $K$ of characteristic zero is a composite of blow-ups and blow-downs with smooth centers.
\end{abstract}

\section{Contents}

\begin{tabular}{lll}
\hline 0. & Introduction & 1
\end{tabular}

1. Main ideas 2

2. Preliminaries 5

\begin{tabular}{llr}
\hline $3 . \quad$ Stratified toric varieties and semifans & 9
\end{tabular}

\begin{tabular}{lll}
\hline $4 . \quad$ Stratified toroidal varieties & 11
\end{tabular}

\begin{tabular}{lll}
\hline 5. Stable valuations & 29
\end{tabular}

6. Correspondence between toroidal morphisms and canonical subdivisions 35

\begin{tabular}{lll}
\hline 7. & Canonical subdivisions and stable support & 38
\end{tabular}

8. Orientability of stratified toroidal varieties and resolution of singularities 45

\begin{tabular}{lll}
\hline 9. & Orientation of toroidal modifications & 49
\end{tabular}

\begin{tabular}{lll}
\hline 10. & The $\pi$-desingularization lemma of Morelli & 52
\end{tabular}

\begin{tabular}{lll}
\hline $11 . \quad$ Birational cobordisms & 62
\end{tabular}

$\begin{array}{lll}12 . & \text { Weak factorization theorem } & 64\end{array}$

\begin{tabular}{lll}
\hline 13. & Comparison with another proof. Torification and stable support & 66
\end{tabular}

\begin{tabular}{ll} 
References & 68 \\
\hline
\end{tabular}

\section{INTRODUCTION}

The main goal of the present paper is two-fold. First we extend the theory of toroidal embeddings introduced by Kempf, Knudsen, Mumford, and Saint-Donat to the class of toroidal varieties with stratifications. Second we give a proof of the following weak factorization conjecture as an application and illustration of the theory.

\section{Conjecture 0.0.1. The Weak Factorization conjecture}

1. Lef $f: X \rightarrow Y$ be a birational map of smooth complete varieties over an algebraically closed field of characteristic zero, which is an isomorphism over an open set $U$. Then $f$ can be factored as

$$
X=X_{0} \stackrel{f_{0}}{\rightarrow} X_{1} \stackrel{f_{1}}{\rightarrow} \ldots \stackrel{f_{n-1}}{\rightarrow} X_{n}=Y,
$$

where each $X_{i}$ is a smooth complete variety and $f_{i}$ is a blow-up or blow-down at a smooth center which is an isomorphism over $U$.

2. Moreover, if $X \backslash U$ and $Y \backslash U$ are divisors with normal crossings, then each $D_{i}:=X_{i} \backslash U$ is a divisor with normal crossings and $f_{i}$ is a blow-up or blow-down at a smooth center which has normal crossings with components of $D_{i}$.

Date: February 19, 2018.

The author was supported in part by Polish KBN grant 2 P03 A 00516 and NSF grant DMS-0100598. 
JAROSŁAW WŁODARCZYK

This theorem extends a theorem of Zariski, which states that any birational map between two smooth complete surfaces can be factored into a succession of blow-ups at points followed by a succession of blowdowns at points. A stronger version of the above theorem, called the strong factorization conjecture remains open.

Conjecture 0.0.2. Strong factorization conjecture. Any birational map $f: X-\rightarrow Y$ of smooth complete varieties can be factored into a succession of blow-ups at smooth centers followed by a succession of blow-downs at smooth centers.

One can find the statements of both conjectures in many papers. Hironaka [29] formulated the strong factorization conjecture. The weak factorization problem was stated by Miyake and Oda [46]. The toric versions of the strong and weak factorizations were also conjectured by Miyake and Oda 46 and are called the strong and weak Oda conjectures. The 3-dimensional toric version of the weak form was established by Danilov [21] (see also Ewald [24]). The weak toric conjecture in arbitrary dimensions was proved in [72] and later independently by Morelli [49], who proposed a proof of the strong factorization conjecture (see also Morelli 50]). Morelli's proof of the weak Oda conjecture was completed, revised and generalized to the toroidal case by Abramovich, Matsuki and Rashid in [5]. A gap in Morelli's proof of the strong Oda conjecture, which was not noticed in [5], was later found by K. Karu.

The local version of the strong factorization problem was posed by Abhyankar in dimension 2 and by Christensen in general, who has solved it for 3-dimensional toric varieties [13. The local version of the weak factorization problem (in characteristic 0) was solved by Cutkosky in [16], who also showed that Oda's strong conjecture implies the local version of the strong conjecture for proper birational morphisms [17] and proved the local strong factorization conjecture in dimension 3 (《17) via Christensen's theorem.

Shortly after the present proof was found, another proof of the weak factorization conjecture was conceived by Abramovich, Karu, Matsuki and the author [4]. Unlike the proof in [4 the present proof does not refer to the weak factorization theorem for toric varieties.

Another application of the theory of stratified toroidal varieties is given in Section 8.3, where we show the existence of a resolution of singularities of toroidal varieties in arbitrary characteristic by blowing up ideals determined by valuations (see Theorem 8.3.2).

All schemes and varieties in the present paper are considered over an algebraically closed field $K$. The assumption of characteristic 0 is needed for the results in Sections 11.4 and 12.4 only, where we use Hironaka's canonical resolution of singularities and canonical principalization for the second part of the theorem (see Hironaka [29], Villamayor [71] and Bierstone-Milman [8]).

\section{MAIN IDEAS}

1.1. Toroidal embeddings. The theory of toroidal embeddings was introduced and developed by Kempf, Knudsen, Mumford and Saint-Donat in [39]. Although it was originally conceived for the purpose of compactifying symmetric spaces and certain moduli spaces it has been successfully applied to various problems concerning resolution of singularities and factorization of morphisms (see [39], [2], 3]). It is an efficient tool in many situations, turning complicated or even "hopeless" algebro-geometric problems into relatively "easy" combinatorial problems. Its significance lies in a simple combinatorial description of the varieties considered carrying rich and precise information about singularities and stratifications.

By a toroidal embedding (originally a toroidal embedding without self-intersections) we mean a variety $X$ with an open subset $U$ with the following property: for any $x \in X$ there is an open neighborhood $U_{x}$ and an étale morphism $\phi: U_{x} \rightarrow X_{\sigma_{x}}$ into a toric variety $X_{\sigma_{x}}$ containing a torus $T$ such that $U_{x} \cap U=\phi^{-1}(T)$. Such a morphism is called a chart.

Let $D_{i}, i \in I$, be the irreducible components of the complement divisor $D=X \backslash U$. We define a stratification $S$ on $X$ with strata $s \in S$ such that either $\bar{s}$ is an irreducible component of $\bigcap_{i \in J} D_{i}$ for some $J \subset I$ or $\bar{s}=X$. The strata are naturally ordered by the following relation: $s \leq s^{\prime}$ iff the closure $\bar{s}$ contains $s^{\prime}$. In particular $s=\bar{s} \backslash \bigcup_{s^{\prime}>s} \overline{s^{\prime}}$. Any chart $\phi: U_{x} \rightarrow X_{\sigma_{x}}$ preserves strata: as a matter of fact, all strata $s \subset U_{x}$ for $s \in S$ are preimages of $T$-orbits on $X_{\sigma_{s}}$. In fact a toroidal embedding can be defined as a stratified variety such that for any point $x \in X$ there exists a neighborhood $U_{x}$ of $x$ and an étale morphism $\phi: U_{x} \rightarrow X_{\sigma_{x}}$ preserving strata.

The cone $\sigma_{x}$ in the above definition can be described using Cartier divisors. Let $x$ belong to a stratum $s \in S$. We associate with $s \in S$ the following data ([39): 
$\mathbf{M}^{s}$ : the group of Cartier divisors in $\operatorname{Star}(s, S):=\bigcup_{s^{\prime} \leq s} s^{\prime}$, supported in $\operatorname{Star}(s, S) \backslash U$,

$\mathbf{N}_{s}:=\operatorname{Hom}\left(\mathbf{M}_{s}, \mathbb{Z}\right)$,

$\mathbf{M}_{+}^{s} \subset \mathbf{M}^{s}$ : effective Cartier divisors,

$\sigma_{s} \subset \mathbf{N}_{\mathbb{R}}^{s}$ : the dual of $\mathbf{M}_{+}^{s}$.

This gives a correspondence between strata $s \in S$ and cones. If $s^{\prime} \leq s$ then $\sigma_{s^{\prime}}$ is a face of $\sigma_{s}$. By glueing cones $\sigma_{s}$ along their subfaces we construct a conical complex $\Sigma$. In contrast to in the standard theory of complexes two faces might share a few common faces, not just one. For any stratum $s \in S$ the integral vectors in the relative interior of $\sigma_{s}$ define monomial valuations on $X_{\sigma_{s}}$. These valuations induce, via the morphisms $\phi$ monomial valuations centered at $\bar{s}$ (independent of charts).

The natural class of morphisms of toroidal embeddings are called toroidal morphisms and are exactly those which are locally determined by charts $\phi$ and toric morphisms:

A birational morphism of toroidal embeddings $f:(Y, U) \rightarrow(X, U)$ is toroidal if for any $x \in s \subset X$ there exists a chart $x \in U_{x} \rightarrow X_{\Delta^{\sigma}}$ and a subdivision $\Delta^{\sigma}$ of $\sigma_{s}$ and a fiber square of morphisms of stratified varieties

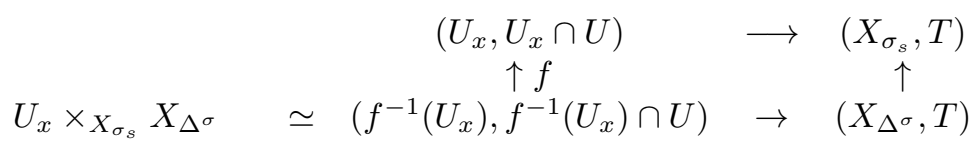

Note that toroidal morphisms are well defined and do not depend upon the choice of the charts. This fact can be described nicely using the following Hironaka condition:

For any points $x, y$ which are in the same stratum every isomorphism $\alpha: \widehat{X}_{x} \rightarrow \widehat{X}_{y}$ preserving stratification can be lifted to an isomorphism $\alpha^{\prime}: Y \times_{X} \widehat{X}_{x} \rightarrow Y \times_{X} \widehat{X}_{y}$ preserving stratification.

Birational toroidal morphisms with fixed target are in bijective correspondence with subdivisions of the associated complex.

1.2. Stratified toroidal varieties. A more general class of objects occurring naturally in applications is the class of stratified toroidal varieties defined as follows.

A stratified toric variety is a toric variety with an invariant equisingular stratification. The important difference between toric varieties and stratified toric varieties is that the latter come with a stratification which may be coarser than the one given by orbits. As a consequence, the combinatorial object associated to a stratified toric variety, called a semifan, consists of those faces of the fan of the toric variety corresponding to strata. The faces which do not correspond to strata are ignored (see Definition 3.1.5).

By a stratified toroidal variety we mean a stratified variety $(X, S)$ such that for any $x \in s, s \in S$ there is an étale map called a chart $\phi_{x}: U_{x} \rightarrow X_{\sigma}$ from an open neighborhood to a stratified toric variety such that all strata in $U_{x}$ are preimages of strata in $X_{\sigma}$ (see Definition 4.1.5). A toroidal embedding is a particular example of a stratified toroidal variety if we consider the stratification described in section 1.1. (see also Definition 4.1.8).

Each chart associates with a stratum $s$ the semicone $\sigma$ i.e the semifan consisting of faces of $\sigma$ corresponding to strata in $\operatorname{Star}(\mathrm{s}, \mathrm{S})$ for the corresponding stratum $s$.

If one chart associates to a stratum a semicone $\sigma_{1}$, and another associates $\sigma_{2}$ then Demushkin's theorem says that $\sigma_{1} \simeq \sigma_{2}$.

This gives us the correspondence between strata and cones with semifans. Consequently, if $s \leq s^{\prime}$, then $\sigma$ is a face of $\sigma^{\prime}$ and the semicone $\sigma$ is a subset of the semicone $\sigma^{\prime}$. By glueing the semicones $\sigma$ and $\sigma^{\prime}$ along the common faces $\sigma^{\prime \prime}$ we obtain the so called semicomplex $\Sigma$ associated to the stratified toroidal variety. The semicomplex is determined uniquely up to isomorphism. Its faces of the semicomplex are indexed by the stratification. If a stratified toroidal variety is a toroidal embedding then the associated semicomplex is the usual conical complex.

Note that in contrast in the case of toroidal embeddings only some integral vectors $v$ in faces of this semicomplex determine unique valuations on $X$ which do not depend upon a chart. These vectors are called stable and the valuations they induce are also called stable (see Definitions 5.3.1, 5.3.2). The stable vectors form a subset in the support of the semicomplex, which we call the stable support (see Definition 6.1.1). 
In particular, let $X$ be a smooth surface and $S$ be its stratification consisting of a point $p \in X$ and its complement. The associated semicomplex consists of the regular cone $\mathbf{Q}_{\geq 0} \cdot e_{1}+\mathbf{Q}_{\geq 0} \cdot e_{2}$ corresponding to the point $p$ and of the vertex corresponding to a big stratum. The only stable valuation is the valuation of the point $p$ which corresponds to the unique (up to proportionality) stable vector $e_{1}+e_{2}$.

The notion of stable support plays a key role in the theory of stratified toroidal varieties. The stable support has many nice properties. It intersects the relative interiors of all faces of the semicomplex and this intersection is polyhedral. The relative interiors of faces which are not in the semicomplex do not intersect the stable support. Consequently, all stable valuations are centered at the closures of strata. The part of the stable support which is contained in a face of the semicomplex is convex and is a union of relative interiors of a finite number of polyhedral cones. Also some "small" vectors like those which are in the parallelogram determined by ray generators belong to the stable support. If we consider the canonical resolution of toric singularities then all divisors occurring in this resolution determine stable valuations. In section 13, where we discuss another approach to the weak factorization conjecture in [4, we also show a method of computing the stable support which is based on the idea of torification of Abramovich and de Jong [2].

The stability condition for vectors in a face $\sigma$ of the semicomplex $\Sigma$ can be expressed in terms of invariance under the action of the group $G^{\sigma}$ of automorphisms of a scheme $\widehat{X}_{\sigma}$ associated to this face (see Definition 5.3.1) (see section 4.13). The vector $v$ is stable if the valuation determined by this vector on $\widetilde{X}_{\sigma}$ is $G^{\sigma}$ invariant. (Definition 5.3.2).

Birational toroidal mophisms or simply toroidal morphisms between stratified toroidal varieties are those induced locally by toric morphisms via toric charts and satisfying the Hironaka condition (see Definition 4.12.2). The Hironaka condition implies that toroidal morphisms, which are a priori induced by toric charts, in fact do not depend upon the charts and depend eventually only on the subdivisions of the semicomplex associated to the original stratified toroidal variety.

This leads to a 1-1 correspondence between toroidal morphisms and certain subdivisions, called canonical, of the semicomplex associated to the original variety (see Theorems 4.14.1 and 6.6.1).

The canonical subdivisions are those satisfying the following condition: all "new" rays of the subdivision are in the stable support (see Proposition 7.6.1). It is not difficult to see the necessity of the condition since the "new" rays correspond to invariant divisors which determine invariant valuations. It is more complicated to show the sufficiency of the condition.

Let $(Y, R) \rightarrow(X, S)$ be a toroidal morphism of stratified toroidal varieties associated to the canonical subdivision $\Delta$ of the semicomplex $\Sigma$ associated to $(X, S)$. Then the semicomplex $\Sigma_{R}$ associated to $(Y, R)$ consists of all faces in $\Delta$ whose relative interiors intersect the stable support of $\Sigma$ (see Theorem 6.6.1).

Toroidal morphisms between stratified toroidal varieties generalize those defined for toroidal embeddings. In that case, all subdivisions are canonical and we get the correspondence between toroidal embeddings and subdivisions of associated semicomplexes mentioned in Section 1.1 above.

In our considerations we require some condition, called orientability, of compatibility of charts on stratified toroidal varieties. This condition is analogous to the orientability of charts on an oriented differentiable manifold. It says that two étale charts $\phi: U \rightarrow X_{\sigma_{s}}$ and $\phi^{\prime}: U \rightarrow X_{\sigma_{s}}$ define an automorphism $\widehat{\phi}^{\prime} \circ \widehat{\phi}^{-1}$ in the identity component of the group of automorphisms of $\operatorname{Spec}\left(\widehat{\mathcal{O}}_{X_{\sigma}}\right)$ (see Definition 4.9.1). In the differential setting, the group of local automorphisms consists of two components. In the algebraic situation the number of components depends upon the singularities. It is finite for toric singularities. Smooth toroidal varieties are oriented, since the group of automorphisms of the completion of a regular local ring is connected.

Let $X$ be a toroidal variety with isolated singularity $x_{1} \cdot x_{2}=x_{3} \cdot x_{4}$ or equivalently after some change of coordinates $y_{1}^{2}+y_{1}^{2}+y_{3}^{2}+y_{4}^{2}=0$. In this case the group of automorphisms of the formal completion of $X$ at the singular point consists of two components. Consequently, there are two orientations on $X$ (for more details see Example 4.10.7).

Toroidal embeddings are oriented stratified toroidal varieties (see Example 4.10.6). Stratified toroidal varieties are orientable (see Section 8.2).

1.3. Toroidal varieties with torus action. Let $\Gamma$ denote any algebraic subgroup of an algebraic torus $T:=K^{*} \times \ldots \times K^{*}$. If $\Gamma$ acts on a stratified toric variety via a group homomorphism $\Gamma \rightarrow T$, then we require additionally, that the stabilizers at closed points in a fixed stratum $s$ are all the same and equal to a group $\Gamma_{s}$ associated to $s$, and there is a $\Gamma_{s}$-equivariant isomorphism of the local rings at these points. 
A $\Gamma$-stratified toroidal variety is a stratified toroidal variety with a $\Gamma$-action which is locally $\Gamma$-equivariantly étale isomorphic to a $\Gamma$-stratified toric variety. We associate with it a $\Gamma$-semicomplex which is the semicomplex $\Sigma$ with associated groups $\Gamma_{\sigma} \subset \Gamma$ acting on $X_{\sigma}$.

$\Gamma$-semicomplexes determine local projections $\pi: \sigma \rightarrow \sigma^{\Gamma}$ defined by good quotients $X_{\sigma_{s}} \rightarrow X_{\sigma_{s}^{\Gamma}}:=X_{\sigma} / \Gamma_{\sigma}$. These local projections are coherent in the sense that they commute with face restrictions and subdivisions.

All the definitions in this category are adapted from the theory of stratified toroidal varieties. We additionally require that charts, morphisms and automorphisms occurring in the definitions are $\Gamma_{\sigma}$ or $\Gamma$-equivariant.

1.4. Birational cobordisms, Morse theory and polyhedral cobordisms of Morelli. The theory of birational cobordisms, which was developed in [73], was inspired by Morelli's proof of the weak factorization theorem for toric varieties, where the notion of combinatorial cobordism was introduced (see [49]).

By a birational cobordism between birational varieties $X$ and $X^{\prime}$ we understand a variety $B$ with the action of the multiplicative group $K^{*}$ such that the "lower boundary" $B_{-}$of $B$ (resp. the "upper boundary" $\left.B_{+}\right)$is an open subset which consists of orbits with no limit at 0 (resp. $\infty$ ). The cobordant varieties $X$ and $X^{\prime}$ are isomorphic to the spaces of orbits (geometric quotients) $B_{-} / K^{*}$ and $B_{+} / K^{*}$ respectively. In differential cobordism the action of the 1-parameter group of diffeomorphisms $G \simeq(\mathbf{R},+) \simeq\left(\mathbf{R}^{*}, \cdot\right)$, defined by the gradient field of a Morse function gives us an analogous interpretation of Morse theory. The bottom and top boundaries determined by a Morse function are isomorphic to the spaces of all orbits with no limit at $-\infty$ and $+\infty$ respectively (or 0 and $+\infty$ in multiplicative notation). The critical points of the Morse function are the fixed points of the action. "Passing through" these points induces simple birational transformations analogous to spherical transformations in differential geometry. If $B$ is a smooth cobordism, then these birational transformations locally replace one weighted projective space with a complementary weighted projective space. These birational transformations can be nicely described using the language of toric varieties and associated fans. The semiinvariant parameters in the neighbouhood of a fixed point provide a chart which is a locally analytic isomorphism of the tangent spaces with the induced linear action. Hence locally, the cobordism is isomorphic to an affine space with a linear hyperbolic action. Locally we can identify $B$ with an affine space $\mathbf{A}^{n}$, which is a toric variety corresponding to a regular cone $\sigma$ in the vector space $N^{\mathbf{Q}}$. The action of $K^{*}$ determines a 1-parameter subgroup, hence an integral vector $v \in N^{\mathbf{Q}}$. The sets $B_{-}$and $B_{+}$correspond to the sets $\sigma_{+}$and $\sigma_{-}$of all faces of $\sigma$ visible from above or below (with respect to the direction of $v$ ), hence to the "upper" and "lower" boundaries of $\sigma$. The vector $v$ defines a projection $\pi: N^{\mathbf{Q}} \rightarrow N^{\mathbf{Q}} / \mathbf{Q} v$. The quotient spaces $B_{-} / K^{*}, B_{+} / K^{*}$ correspond to two "cobordant" (in the sense of Morelli) subdivisions $\pi\left(\sigma_{+}\right)$and $\pi\left(\sigma_{-}\right)$of the cone $\pi(\sigma)$. The problem lies in the fact that the fans $\pi\left(\sigma_{+}\right)$, $\pi\left(\sigma_{-}\right)$are singular (which means that they are not spanned by a part of an integral basis). Consequently, the corresponding birational transformation is a composition of weighted blow-ups and blow-downs between singular varieties. The process of resolution of singularities of the quotient spaces is called $\pi$-desingularization and can be achieved locally by a combinatorial algorithm. The difficulty is now in patching these local $\pi$ desingularizations. This problem can be solved immediately once the theory of stratified toroidal varieties (with $K^{*}$-action) is established.

1.5. Sketch of proof. Let $B=B\left(X, X^{\prime}\right)$ be a smooth cobordism between smooth varieties $X$ and $X^{\prime}$. Let $S$ be the stratification determined by the isotropy groups. Then $(B, S)$ is a stratified toroidal variety with $K^{*}$-action. We can associate with it a $K^{*}$-semicomplex $\Sigma$. The $K^{*}$-semicomplex $\Sigma$ determines for any face $\sigma_{s} \in \Sigma$ a projection $\pi_{\sigma}: \sigma \rightarrow \sigma^{\Gamma}$ determined by the good quotients $X_{\sigma} \rightarrow X_{\sigma} / \Gamma_{\sigma}=X_{\sigma}^{\Gamma}$. We apply a combinatorial algorithm - the so called $\pi$-desingularization Lemma of Morelli - to the semicomplex associated to the cobordism. The algorithm consists of starf subdivisions at stable vectors. Such subdivisions are canonical. As a result we obtain a semicomplex with the property that the projections of all simplices are either regular (nonsingular) cones or cones of smaller dimension (than the dimension of the projected cones) (see Lemma 10.4.4 for details). The semicomplex corresponds to a $\pi$-regular toroidal cobordism, all of whose open affine fixed point free subsets have smooth geometric quotients. The existence of such a cobordism easily implies the weak factorization theorem. (The blow-ups, blow-downs and flips induced by elementary cobordisms are regular (smooth)). Since each flip is a composition of a blow-up and a blow-down at a smooth center we come to a factorization of the map $X-\rightarrow X^{\prime}$ into blow-ups and blow-downs at smooth centers (see Proposition 12.2.1).

\section{Preliminaries}


2.1. Basic notation and terminology. Let $N \simeq \mathbf{Z}^{k}$ be a lattice contained in the vector space $N^{\mathbf{Q}}:=$ $N \otimes \mathbf{Q} \supset N$. By a cone in this paper we mean a convex set $\sigma=\mathbf{Q}_{\geq 0} \cdot v_{1}+\ldots+\mathbf{Q}_{\geq 0} \cdot v_{k} \subset N^{\mathbf{Q}}$. By abuse of language we shall speak of a cone $\sigma$ in a lattice $N$. To avoid confusion we shall sometimes write $(\sigma, N)$ for the cone $\sigma$ in $N$. For a cone $\sigma$ in $N$ denote by $N_{\sigma}:=N \cap \operatorname{lin}(\sigma)$ the sublattice generated by $\sigma$. Then by

$$
\underline{\sigma}:=\left(\sigma, N_{\sigma}\right)
$$

we denote the corresponding cone in $N_{\sigma}$. (Sometimes the cones $\sigma$ and $\underline{\sigma}$ will be identified).

We call a vector $v \in N$ primitive if it generates sublattice $\mathbf{Q}_{\geq 0} v_{i} \cap N$. Each ray $\rho \in N^{\mathbf{Q}}$ contains a unique primitive ray $\operatorname{prim}(\rho)$. If $v_{1}, \ldots, v_{k}$ form a minimal set of primitive vectors generating $\sigma \subset N^{\mathbf{Q}}$ in the above sense, then we write

$$
\sigma=\left\langle v_{1}, \ldots, v_{k}\right\rangle .
$$

If $\sigma$ contains no line we call it strictly convex. All cones considered in this paper are strictly convex. For any $\sigma$ denote by $\operatorname{lin}(\sigma)$ the linear span of $\sigma$. For any cones $\sigma_{1}$ and $\sigma_{2}$ in $N$ we write

$$
\sigma=\sigma_{1}+\sigma_{2}
$$

if $\sigma=\left\{v_{1}+v_{2} \mid v_{1} \in \sigma_{1}, v_{2} \in \sigma_{2}\right\}$, and

$$
\sigma=\sigma_{1} \oplus \sigma_{2}
$$

if $\operatorname{lin}\left(\sigma_{1}\right) \cap \operatorname{lin}\left(\sigma_{2}\right)=\{0\}$ and for any $v \in \sigma \cap N$ there exist $v_{1} \in \sigma_{1} \cap N$ and $v_{2} \in \sigma_{2} \cap N$ such that $v=v_{1}+v_{2}$. For any cones $\sigma_{1}$ in $N_{1}$ and $\sigma_{2}$ in $N_{2}$ we define the cone $\sigma_{1} \times \sigma_{2}$ in $N_{1} \times N_{2}$ to be

$$
\sigma_{1} \times \sigma_{2}:=\left\{\left(v_{1}, v_{2}\right) \mid v_{i} \in \sigma_{i} \quad \text { for } \quad i=1,2\right\} .
$$

We say that a cone $\sigma$ in $N$ is regular if there exist vectors $e_{1}, . ., e_{k} \in N$ such that

$$
\sigma=\left\langle e_{1}\right\rangle \oplus \ldots \oplus\left\langle e_{k}\right\rangle .
$$

A cone $\sigma$ is simplicial if $\sigma=\left\langle v_{1}, \ldots, v_{k}\right\rangle$ is generated by linearly independent vectors. We call $\sigma$ indecomposable if it cannot be represented as $\sigma=\sigma^{\prime} \oplus\langle e\rangle$ for some nonzero vector $e \in N$.

Lemma 2.1.1. Any cone $\sigma$ in $N$ is uniquely represented as

$$
\sigma=\operatorname{sing}(\sigma) \oplus\left\langle e_{1}, \ldots, e_{k}\right\rangle,
$$

where $\left\langle e_{1}, \ldots, e_{k}\right\rangle$ is a regular cone and $\operatorname{sing}(\sigma)$ is the maximal indecomposable face of $\sigma$.

By int $(\sigma)$ we denote the relative interior of $\sigma$.

For any simplicial cone $\sigma=\left\langle v_{1}, \ldots, v_{k}\right\rangle$ in $N$ set

$$
\begin{aligned}
& \operatorname{par}(\sigma):=\left\{v \in \sigma \cap N_{\sigma} \mid v=\alpha_{1} v_{1}+\ldots+\alpha_{k} v_{k}, \text { where } 0 \leq \alpha_{i}<1\right\}, \\
& \overline{\operatorname{par}(\sigma)}:=\left\{v \in \sigma \cap N_{\sigma} \mid v=\alpha_{1} v_{1}+\ldots+\alpha_{k} v_{k}, \text { where } 0 \leq \alpha_{i} \leq 1\right\} .
\end{aligned}
$$

For any simplicial cone $\sigma=\left\langle v_{1}, \ldots, v_{k}\right\rangle$ in $N$, by $\operatorname{det}(\sigma)$ we mean $\operatorname{det}\left(v_{1}, \ldots, v_{k}\right)$, where all vectors are considered in some basis of $N \cap \operatorname{lin}(\sigma)$ (a change of basis can only change the sign of the determinant).

Definition 2.1.2. A minimal generator of a cone $\sigma$ is a vector not contained in a one dimensional face of $\sigma$ and which cannot be represented as the sum of two nonzero integral vectors in $\sigma$. A minimal internal vector of a cone $\sigma$ is a vector in $\operatorname{int}(\sigma)$ which cannot be represented as the sum of two nonzero integral vectors in $\sigma$ such that at least one of them belongs to $\operatorname{int}(\sigma)$.

Immediately from the definition we get

Lemma 2.1.3. Any minimal generator $v$ of $\sigma$ is a minimal internal vector of the face $\sigma_{v}$ of $\sigma$, containing $v$ in its relative interior.

Lemma 2.1.4. For any simplicial $\sigma$ each vector from $\operatorname{par}(\sigma)$ can be represented as a nonnegative integral combination of minimal generators. 


\subsection{Toric varieties.}

Definition 2.2.1. (see [20], [56]). By a $\operatorname{fan} \Sigma$ in $N$ we mean a finite collection of finitely generated strictly convex cones $\sigma$ in $N$ such that

- any face of a cone in $\Sigma$ belongs to $\Sigma$,

- any two cones of $\Sigma$ intersect in a common face.

By the support of the fan we mean the union of all its faces, $|\Sigma|=\bigcup_{\sigma \in \Sigma} \sigma$.

If $\sigma$ is a face of $\sigma^{\prime}$ we shall write $\sigma \preceq \sigma^{\prime}$.

If $\sigma \preceq \sigma^{\prime}$ but $\sigma \neq \sigma^{\prime}$ we shall write $\sigma \prec \sigma^{\prime}$.

For any set of cones $\Sigma$ in $N$ by $\bar{\Sigma}$ we denote the set $\left\{\tau \mid \tau \prec \tau^{\prime}\right.$ for some $\left.\tau^{\prime} \in \Sigma\right\}$

Definition 2.2.2. Let $\Sigma$ be a fan and $\tau \in \Sigma$. The star of the cone $\tau$ and the closed star of $\Sigma$ are defined as follows:

$$
\begin{gathered}
\operatorname{Star}(\tau, \Sigma):=\{\sigma \in \Sigma \mid \tau \preceq \sigma\}, \\
\overline{\operatorname{Star}}(\tau, \Sigma):=\left\{\sigma \in \Sigma \mid \sigma^{\prime} \preceq \sigma \text { for some } \sigma^{\prime} \in \operatorname{Star}(\tau, \Sigma)\right\},
\end{gathered}
$$

Definition 2.2.3. Let $\Sigma_{i}$ be a fan in $N_{i}$ for $i=1,2$. Then the product of $\Sigma_{1}$ and $\Sigma_{2}$ is a fan $\Sigma_{1} \times \Sigma_{2}$ in $N_{1}^{\mathbf{Q}} \times N_{2}^{\mathbf{Q}}$ defined as follows:

$$
\Sigma_{1} \times \Sigma_{2}:=\left\{\sigma_{1} \times \sigma_{2} \mid \sigma_{1} \in \Sigma_{1}, \sigma_{2} \in \Sigma_{2}\right\}
$$

To a fan $\Sigma$ there is associated a toric variety $X_{\Sigma} \supset T$, i.e. a normal variety on which a torus $T$ acts effectively with an open dense orbit (see [39], 21], [56], 25]). To each cone $\sigma \in \Sigma$ corresponds an open affine invariant subset $X_{\sigma}$ and its unique closed orbit $O_{\sigma}$. The orbits in the closure of the orbit $O_{\sigma}$ correspond to the cones of $\operatorname{Star}(\sigma, \Sigma)$.

Denote by

$$
M:=\operatorname{Hom}_{\text {alg.gr. }}\left(T, K^{*}\right)
$$

the lattice of group homomorphisms to $K^{*}$, i.e. characters of $T$. Then the dual lattice $N=\operatorname{Hom}_{\text {alg.gr. }}\left(K^{*}, T\right)$ can be identified with the lattice of 1-parameter subgroups of $T$. Then vector space $M^{\mathbf{Q}}:=M \otimes \mathbf{Q}$ is dual to $N^{\mathbf{Q}}:=N \otimes \mathbf{Q}$. Let $(v, w)$ denote the relevant pairing for $v \in N, w \in M$

For any $\sigma \subset N^{\mathbf{Q}}$ we denote by

$$
\sigma^{\vee}:=\{m \in M \mid(v, m) \geq 0 \text { for any } m \in \sigma\}
$$

the set of integral vectors of the dual cone to $\sigma$. Then the ring of regular functions $K\left[X_{\sigma}\right]$ is $K\left[\sigma^{\vee}\right]$.

Each vector $v \in N$ defines a linear function on $M$ which determines a valuation $\operatorname{val}(v)$ on $X_{\Sigma}$.

For any regular function $f=\sum_{w \in M} a_{w} x^{w} \in K[T]$ set

$$
\operatorname{val}(v)(f):=\min \left\{(v, w) \mid a_{w} \neq 0\right\} .
$$

Thus $N$ can be perceived as the lattice of all $T$-invariant integral valuations of the function field of $X_{\Sigma}$.

For any $\sigma \subset N^{\mathbf{Q}}$ set

$$
\sigma^{\perp}:=\{m \in M \mid(v, m)=0 \text { for any } m \in \sigma\} .
$$

The latter set represents all invertible characters on $X_{\sigma}$. All noninvertible characters are 0 on $O_{\sigma}$. The ring of regular functions on $O_{\sigma} \subset X_{\sigma}$ can be written as $K\left[O_{\sigma}\right]=K\left[\sigma^{\perp}\right] \subset K\left[\sigma^{\vee}\right]=K\left[\sigma^{\perp}\right] \otimes K[\underline{\sigma}]$. Thus $K\left[X_{\sigma}\right]=K\left[O_{\sigma}\right]\left[\underline{\sigma}^{\vee}\right]$

Let $T_{\sigma} \subset T$ be the subtorus corresponding the sublattice $N_{\sigma}:=\operatorname{lin}(\sigma) \cap N$ of $N$. Then by definition, $T_{\sigma}$ acts trivially on $K\left[O_{\sigma}\right]=K\left[\sigma^{\perp}\right]=K\left[X_{\sigma}\right]^{T_{\sigma}}$. Thus $T_{\sigma}=\left\{t \in T \mid t x=x, x \in O_{\sigma}\right\}$.

This leads us to the lemma

Lemma 2.2.4. Any toric variety $X_{\sigma}$ is isomorphic to $X_{\underline{\sigma}} \times O_{\sigma}$, where $O_{\sigma} \simeq T / T_{\sigma}$.

For any $\sigma \in \Sigma$ the closure $\bar{O}_{\sigma}$ of the orbit $O_{\sigma} \subset X_{\Sigma}$ is a toric variety with the big torus $T / T_{\sigma}$. Let $\pi: N \rightarrow N / N_{\sigma}$ be the natural projection. Then $\bar{O}_{\sigma}$ corresponds to the fan $\Sigma^{\prime}:=\left\{\frac{\tau+\operatorname{lin}(\sigma)}{\operatorname{lin}(\sigma)} \mid \tau \in \operatorname{Star}(\tau, \Sigma)\right\}$ in $N / N_{\sigma}$. 


\subsection{Morphisms of toric varieties.}

Definition 2.3.1. (see 39], [56], 21], 25). A birational toric morphism or simply a toric morphism of toric varieties $X_{\Sigma} \rightarrow X_{\Sigma^{\prime}}$ is a morphism identical on $T \subset X_{\Sigma}, X_{\Sigma^{\prime}}$

Definition 2.3.2. (see [39], [56], [21], [25]). A subdivision of a fan $\Sigma$ is a fan $\Delta$ such that $|\Delta|=|\Sigma|$ and any cone $\sigma \in \Sigma$ is a union of cones $\delta \in \Delta$.

Definition 2.3.3. Let $\Sigma$ be a fan and $\varrho$ be a ray passing in the relative interior of $\tau \in \Sigma$. Then the star subdivision $\varrho \cdot \Sigma$ of $\Sigma$ with respect to $\varrho$ is defined to be

$$
\varrho \cdot \Sigma=(\Sigma \backslash \operatorname{Star}(\tau, \Sigma)) \cup\{\varrho+\sigma \mid \sigma \in \overline{\operatorname{Star}}(\tau, \Sigma) \backslash \operatorname{Star}(\tau, \Sigma)\} .
$$

If $\Sigma$ is regular, i.e. all its cones are regular, $\tau=\left\langle v_{1}, \ldots, v_{l}\right\rangle$ and $\varrho=\left\langle v_{1}+\ldots+v_{l}\right\rangle$ then we call the star subdivision $\varrho \cdot \Sigma$ regular.

Proposition 2.3.4. (see [39], 21], [56], [25]). Let $X_{\Sigma}$ be a toric variety. There is a 1-1 correspondence between subdivisions of the fan $\Sigma$ and proper toric morphisms $X_{\Sigma^{\prime}} \rightarrow X_{\Sigma}$.

Remark. Regular star subdivisions from 2.3.3 correspond to blow-ups of smooth varieties at closures of orbits ([56], [25]). Arbitrary star subdivisions correspond to blow-ups of some ideals associated to valuations (see Lemma 5.2.8).

2.4. Toric varieties with $\Gamma$-action. In further considerations let $\Gamma$ denote any algebraic subgroup of an algebraic torus $T=K^{*} \times \ldots \times K^{*}$. By $X / \Gamma$ and $X / / \Gamma$ we denote respectively geometric and good quotients. If $\Gamma$ acts on an algebraic variety $X$ and $x \in X$ is a closed point, then $\Gamma_{x}$ denotes the isotropy group of $x$.

Definition 2.4.1. Let $\Gamma$ act on $X_{\sigma} \supset T$ via a group homomomorphism $\Gamma \rightarrow T$. Set

$$
\Gamma_{\sigma}:=\left\{g \in \Gamma \mid g(x)=x \text { for any } x \in O_{\sigma}\right\} .
$$

We shall write

$$
\sigma=\sigma^{\prime} \oplus^{\Gamma}\left\langle e_{1}, \ldots, e_{k}\right\rangle
$$

if $\sigma=\sigma^{\prime} \oplus\left\langle e_{1}, \ldots, e_{k}\right\rangle,\left\langle e_{1}, \ldots, e_{k}\right\rangle$ is regular and $\Gamma_{\sigma}=\Gamma_{\sigma^{\prime}}$. We say that $\sigma$ is $\Gamma$-indecomposable if it cannot be represented as $\sigma=\sigma^{\prime} \oplus^{\Gamma}\left\langle e_{1}, \ldots, e_{k}\right\rangle$ (or equivalently $X_{\sigma}$ is not of the form $X_{\sigma^{\prime}} \times \mathbf{A}^{k}$ with $\Gamma_{\sigma}$ acting trivially on $\mathbf{A}^{k}$ ). By

$$
\operatorname{sing}^{\Gamma}(\sigma)
$$

we mean the maximal $\Gamma$-indecomposable face of $\sigma$.

If $\Gamma$ acts on a toric vatiety via a group homomomorphism $\Gamma \rightarrow T$ we shall speak of a toric action of $\Gamma$.

2.5. Demushkin's Theorem. For any algebraic variety $X$ and its (in general nonclosed) point $x \in X$ we denote by $\widehat{\mathcal{O}}_{X, x}$ the completion of the local ring $\mathcal{O}_{X, x}$ at the maximal ideal of $x$. We also set

$$
\widehat{X}_{x}:=\operatorname{Spec}\left(\widehat{\mathcal{O}}_{X, x}\right) \text {. }
$$

For the affine toric variety $X_{\sigma}$ define

$$
\widehat{X}_{\sigma}:=\operatorname{Spec}\left(\widehat{\mathcal{O}}_{X_{\sigma}, O_{\sigma}}\right) \text {. }
$$

We shall use the following Theorem of Demushkin (22]):

Theorem 2.5.1. Let $\sigma$ and $\tau$ be two cones of maximal dimension in isomorphic lattices $N_{\sigma} \simeq N_{\tau}$.

Then the following conditions are equivalent:

1. $\sigma \simeq \tau$

2. $\widehat{X}_{\sigma} \simeq \widehat{X}_{\tau}$.

Proof. For the proof see 22 or the proof of 4.6.1.

The above theorem can be formulated for affine toric varieties with $\Gamma$-action.

Theorem 2.5.2. Let $\sigma$ and $\tau$ be two cones of maximal dimension in isomorphic lattices $N_{\sigma} \simeq N_{\tau}$. Let $\Gamma$ act on $X_{\sigma} \supset T_{\sigma}$ and on $X_{\tau} \supset T_{\tau}$ via group homomorpisms $\Gamma \rightarrow T_{\sigma}$ and $\Gamma \rightarrow T_{\tau}$.

Then the following conditions are equivalent: 
1. There exists an isomorphism of cones $\sigma \simeq \tau$ inducing a $\Gamma$-equivariant isomorphism of toric varieties.

2. There exists a $\Gamma$-equivariant isomorphism $\widehat{X}_{\sigma} \simeq \widehat{X}_{\tau}$.

Proof. For the proof see the proof of 4.6.1 or [22].

Definition 2.5.3. Let $\Gamma$ act on $X_{\sigma} \supset T_{\sigma}$ and on $X_{\tau} \supset T_{\tau}$ via group homomorpisms $\Gamma \rightarrow T_{\sigma}$ and $\Gamma \rightarrow T_{\tau}$. We say that cones $\sigma$ and $\tau$ are $\Gamma$-isomorphic if there exists an isomorphism of cones $\sigma \simeq \tau$ inducing a $\Gamma$-equivariant isomorphism of toric varieties.

The above theorem can be formulated as follows:

Theorem 2.5.4. Let $\sigma$ and $\tau$ be two cones in isomorphic lattices $N_{\sigma} \simeq N_{\tau}$. Let $\Gamma$ act on $X_{\sigma} \supset T_{\sigma}$ and on $X_{\tau} \supset T_{\tau}$ via group homomorpisms $\Gamma \rightarrow T_{\sigma}$ and $\Gamma \rightarrow T_{\tau}$. Assume that $\Gamma_{\sigma}=\Gamma_{\tau}$.

Then the following conditions are equivalent:

1. $\operatorname{sing}^{\Gamma}(\sigma)$ and $\operatorname{sing}^{\Gamma}(\tau)$ are $\Gamma_{\sigma}$-isomorphic.

2. For any closed points $x_{\sigma} \in O_{\sigma}$ and $x_{\tau} \in O_{\tau}$, there exists a $\Gamma_{\sigma}$-equivariant isomorphism of the local rings $\mathcal{O}_{X_{\sigma}, x_{\sigma}}$ and $\mathcal{O}_{X_{\tau}, x_{\tau}}$.

3. For any closed points $x_{\sigma} \in O_{\sigma}$ and $x_{\tau} \in O_{\tau}$, there exists a $\Gamma_{\sigma}$-equivariant isomorphism of the completions of the local rings $\widehat{\mathcal{O}}_{X_{\sigma}, x_{\sigma}}$ and $\widehat{\mathcal{O}}_{X_{\tau}, x_{\tau}}$.

Proof. $(1) \Rightarrow(2)$ Write $\sigma=\operatorname{sing}^{\Gamma}(\sigma) \oplus^{\Gamma} \mathrm{r}(\sigma)$ and $\tau=\operatorname{sing}^{\Gamma}(\tau) \oplus^{\Gamma} \mathrm{r}(\tau)$, where $\mathrm{r}(\sigma)$, $\mathrm{r}(\tau)$ denote regular cones. By Lemma 2.2.4, $X_{\sigma}=X_{\underline{\sigma}} \times O_{\sigma}=X_{\underline{\operatorname{sing} \Gamma(\sigma)}} \times X_{\underline{\mathrm{r}(\sigma)}} \times O_{\sigma}$, where $\Gamma_{\sigma}$ acts trivially on $X_{\mathrm{r}(\sigma)} \times O_{\sigma}$. Thus there exists a $\Gamma_{\sigma^{-}}$-equivariant isomorphism $\mathcal{O}_{X_{\sigma}, x_{\sigma}} \simeq \overline{\mathcal{O}}_{X_{\underline{\operatorname{sing}} \Gamma(\sigma)}} \times X_{\mathrm{r}^{\prime}(\sigma)}$, where $\mathrm{r}^{\prime}(\sigma)$ is a regular cone of $\operatorname{dimension} \operatorname{dim}\left(N_{\sigma}\right)-\operatorname{dim}\left(\operatorname{sing}^{\Gamma}(\sigma)\right)$ and $\Gamma_{\sigma}$ acts trivially on $X_{\mathrm{r}^{\prime}(\sigma)}$. Analogously $\mathcal{O}_{X_{\tau}, x_{\tau}} \simeq \mathcal{O}_{X_{\underline{s i n g}} \Gamma(\tau)} \times X_{\mathrm{r}^{\prime}(\tau)} \simeq$

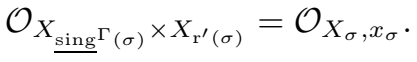

The implication $(2) \Rightarrow(3)$ is trivial. For the proof of $(3) \Rightarrow(1)$ we find a regular $\operatorname{cone} \operatorname{re}(\sigma)$ in $N_{\sigma}$ such that $\sigma^{\prime}:=\sigma \oplus \operatorname{re}(\sigma)$ is of maximal dimension in $N_{\sigma}$. Since $\Gamma_{\sigma}$ acts trivially on $O_{\sigma}$ and on $O_{\tau}$ we have $\sigma^{\prime}:=\sigma \oplus^{\Gamma_{\sigma}} \operatorname{re}(\sigma)$. Analogously $\tau^{\prime}:=\tau \oplus^{\Gamma_{\tau}} \operatorname{re}(\tau)$. By Theorem 2.5.2, $\sigma^{\prime}$ and $\tau^{\prime}$ are $\Gamma_{\sigma}$-isomorphic. Consequently, $\operatorname{sing}^{\Gamma}(\sigma)=\operatorname{sing}^{\Gamma_{\sigma}}\left(\sigma^{\prime}\right) \simeq \operatorname{sing}^{\Gamma_{\tau}}\left(\tau^{\prime}\right)=\operatorname{sing}^{\Gamma}(\tau)$.

Theorem 2.5.4 allows us to assign a singularity type to any closed point $x$ of a toric variety $X$ with a toric action of the group $\Gamma$ :

Definition 2.5.5. By the singularity type of a point $x$ of a toric variety $X$ we mean the function

$$
\operatorname{sing}(x):=\underline{\operatorname{sing}\left(\sigma_{x}\right)}
$$

where $\sigma_{x}$ is a cone of maximal dimension such that $\widehat{X}_{x} \simeq \widehat{X}_{\sigma_{x}}$.

By the singularity type of a point $x$ of a toric variety $X$ with a toric action of group $\Gamma$ we mean

$$
\operatorname{sing}^{\Gamma}(x):=\left(\Gamma_{x}, \underline{\operatorname{sing}^{\Gamma_{x}}\left(\sigma_{x}\right)}\right),
$$

where $\sigma_{x}$ is a cone of maximal dimension with toric action of $\Gamma_{x}$ on $\widehat{X}_{\sigma_{x}}$ and such that there exists a $\Gamma_{x}$-equivariant isomorphism $\widehat{X}_{x} \simeq \widehat{X}_{\sigma_{x}}$.

\section{Stratified TORIC VARIETIES AND SEMIFANS}

3.1. Definition of a stratified toric variety. In this section, we give a combinatorial description of stratified toric varieties in terms of so called (embedded) semifans.

Definition 3.1.1. Let $X$ be a noetherian scheme $X$ over $\operatorname{Spec}(K)$. A stratification of $X$ is a decomposition of $X$ into a finite collection $S$ of pairwise disjoint locally closed irreducible smooth subschemes $s \subset X$, called strata, with the following property: For every $s \in S$, the closure $\bar{s} \subset X$ is a union of strata.

Definition 3.1.2. Let $S$ and $S^{\prime}$ be two stratifications of $X$. We say that $S$ is finer than $S^{\prime}$ if any stratum in $S^{\prime}$ is a union of strata in $S$. In this case we shall also call $S^{\prime}$ coarser than $S$.

Definition 3.1.3. Let $X$ be a toric variety with big torus $T \subset X$. A toric stratification of $X$ is a stratification $S$ of $X$ consisting of $T$-invariant strata $s$ such that for any two closed points $x, x^{\prime} \in s$ their local rings $\mathcal{O}_{X, x}$ and $\mathcal{O}_{X, x^{\prime}}$ are isomorphic. 
Definition 3.1.4. Let $X$ be a toric variety with a toric action of $\Gamma$. We say that a toric stratification $S$ of $X$ is compatible with the action of $\Gamma$ if

1. All points in the same stratum $s$ have the same isotropy group $\Gamma_{s}$.

2. For any two closed points $x, y$ from one stratum $s$ there exists a $\Gamma_{s}$-equivariant isomorphism $\alpha: \widehat{X}_{x} \rightarrow$ $\widehat{X}_{y}$, preserving all strata.

If $S$ is a toric stratification of $X$, then we shall also speak of a stratified toric variety $(X, S)$. If $X$ is a toric variety with a toric stratification, compatible with $\Gamma$, then we shall also speak of a $\Gamma$-stratified toric variety $(X, S)$.

The combinatorial objects we shall use in this context are the following:

Definition 3.1.5. An embedded semifan is a subset $\Omega \subset \Sigma$ of a fan $\Sigma$ in a lattice $N$ such that for every $\sigma \in \Sigma$ there is an $\omega(\sigma) \in \Omega$ satisfying

1. $\omega(\sigma) \preceq \sigma$ and any other $\omega \in \Omega$ with $\omega \preceq \sigma$ is a face of $\omega(\sigma)$,

2. $\sigma=\omega(\sigma) \oplus \mathrm{r}(\sigma)$ for some regular cone $\mathrm{r}(\sigma) \in \Sigma$.

A semifan in a lattice $N$ is a set $\Omega$ of cones in $N$ such that the set $\Sigma$ of all faces of the cones of $\Omega$ is a fan in $N$ and $\Omega \subset \Sigma$ is an embedded semifan.

Definition 3.1.6. An embedded semifan $\Omega \subset \Sigma$ with a toric action of $\Gamma$ on $X_{\Sigma}$ will be called an embedded $\Gamma$-semifan if for every $\sigma \in \Sigma, \sigma=\omega(\sigma) \oplus^{\Gamma} \mathrm{r}(\sigma)$.

Remark. A semifan or an embedded semifan can be viewed as a $\Gamma$-semifan or an embedded $\Gamma$-semifan with trivial group $\Gamma$.

Some examples are discussed at the end of this section. The main statement of this section says that stratified toric varieties are described by embedded semifans:

Proposition 3.1.7. Let $\Sigma$ be a fan in a lattice $N$, and let $X$ denote the associated toric variety with a toric action of $\Gamma$. There is a canonical 1-1 correspondence between the toric stratifications of $X$ compatible withn the action of $\Gamma$ and the embedded $\Gamma$-semifans $\Omega \subset \Sigma$ :

1. If $S$ is a toric stratification of $X$, compatible with the action of $\Gamma$, then the corresponding embedded $\Gamma$-semifan $\Omega \subset \Sigma$ consists of all those cones $\omega \in \Sigma$ that describe the big orbit of some stratum $s \in S$.

2. If $\Omega \subset \Sigma$ is an embedded $\Gamma$-semifan, then the strata of the associated toric stratification $S$ of $X$ arise from the cones of $\Omega$ via

$$
\omega \mapsto \operatorname{strat}(\omega):=\bigcup_{\omega(\sigma)=\omega} O_{\sigma} .
$$

Proof. (1) $\Rightarrow(2)$ Since strata of $S$ are $T$-invariant and disjoint, each orbit $O_{\tau}$ belongs to a unique stratum $s$. Let $\omega \in \Omega$ describe the big open orbit of $s$. Then $O_{\tau}$ is contained in the closure of $O_{\omega}$. Hence $\omega$ is a face of $\tau$. By Definition de: embedded, $\Gamma_{\tau}=\Gamma_{\omega}$ and there exists a $\Gamma_{\tau}$-equivariant isomorphism of the local rings $\mathcal{O}_{X_{\tau}, x}$ and $\mathcal{O}_{X_{\omega}, y}$ of two points $x \in O_{\tau}$ and $y \in O_{\omega}$. By Theorem 2.5.4 and since $\operatorname{sing}^{\Gamma}(\tau) \supset \operatorname{sing}^{\Gamma}(\omega)$, we infer that $\operatorname{sing}^{\Gamma}(\tau)=\operatorname{sing}^{\Gamma}(\omega)$. Hence $\omega=\operatorname{sing}^{\Gamma}(\omega) \oplus^{\Gamma} \mathrm{r}(\omega)$ and $\tau=\operatorname{sing}^{\Gamma}(\tau) \oplus^{\Gamma} \mathrm{r}(\tau)=\omega \oplus^{\Gamma} \mathrm{r}^{\prime}(\tau)$, where $\mathrm{r}(\tau)=\mathrm{r}(\omega) \oplus \mathrm{r}^{\prime}(\tau)$.

$(2) \Rightarrow(1)$. By definition $\{\sigma \in \Sigma \mid \omega(\sigma)=\omega)\}=\operatorname{Star}(\omega, \Sigma) \backslash \bigcup_{\omega \prec \omega^{\prime} \in \Omega} \operatorname{Star}\left(\omega^{\prime}, \Sigma\right)$. Hence all the defined subsets strat $(\omega)$ are locally closed. The closure of each subset $\operatorname{strat}(\omega)$ corresponds to $\operatorname{Star}(\omega, \Sigma)$ and hence it is a union of the sets $\operatorname{strat}\left(\omega^{\prime}\right)$, where $\omega \prec \omega^{\prime}$. Since $\tau=\omega \oplus^{\Gamma} \mathrm{r}(\tau)$ we have $\Gamma_{\tau}=\Gamma_{\omega}$. Each subset $\operatorname{strat}(\omega)$ is a toric variety with a fan $\Sigma^{\prime}:=\left\{\frac{\tau+\operatorname{lin}(\omega)}{\operatorname{lin}(\omega)} \mid \omega(\tau)=\omega\right\}$ in $\left(N^{Q}\right)^{\prime}:=N^{Q} / \operatorname{lin}(\omega)$. Since $\tau=\omega \oplus^{\Gamma} \mathrm{r}(\tau)$ the cone $\frac{\tau+\operatorname{lin}(\omega)}{\operatorname{lin}(\omega)}$ is isomorphic to the regular cone $\mathrm{r}(\tau)$ in $\left(N^{Q}\right)^{\prime}$. Thus the strata strat $(\omega)$ is smooth. The local rings of closed points of the the strata strat $(\omega)$ have the same isotropy group $\Gamma_{\omega}$. Moreover if $O_{\tau} \subset \operatorname{strat}(\omega)$ then $\operatorname{sing}^{\Gamma}(\tau)=\operatorname{sing}^{\Gamma}(\omega)$. By Theorem 2.5.4, we conclude that there exists a $\Gamma$-equivariant isomorphism of the local rings of any two points $x \in O_{\tau}$ and $y \in O_{\omega}$.

As a corollary from the above we obtain the following lemmas:

Lemma 3.1.8. $\operatorname{strat}(\omega)=\overline{\operatorname{strat}(\omega)} \backslash \bigcup_{\omega^{\prime} \prec \omega} \overline{\operatorname{strat}\left(\omega^{\prime}\right)}$. 
Lemma 3.1.9. Let $S_{1}$ and $S_{2}$ be two toric stratifications on a toric variety $X$ corresponding to two embedded semifans $\Omega_{1}, \Omega_{2} \subset \Sigma$. Then $S_{1}$ is coarser than $S_{2}$ iff $\Omega_{1} \subset \Omega_{2}$.

Proof $(\Rightarrow)$ Assume that $S_{1}$ is coarser than $S_{2}$. Let $\omega \in \Omega_{1}$ be the cone corresponding to a stratum $s_{1} \in S_{1}$. The closure $\overline{s_{1}}$ is a union of strata in $S_{2}$. There is a generic stratum $s_{2} \in S_{2}$ contained in $\overline{s_{1}}$. Then $\overline{s_{1}}=\overline{s_{2}}$ and $\omega \in \Omega_{2}$ corresponds to the stratum $s_{2}$.

$(\Leftarrow)$ Now assume that $\Omega_{1} \subset \Omega_{2}$. Then the closure of any stratum $s_{1}$ equals $\overline{s_{1}}=\overline{\omega_{1}}$, where $\omega_{1} \in \Omega_{1}$ is the cone corresponding to $s_{1}$. But then $\overline{\omega_{1}}=\overline{s_{2}}$, where $s_{2} \in S_{2}$ is the stratum corresponding to $\omega_{1} \in \Omega_{2}$. Thus $\overline{s_{1}}=\overline{s_{2}}$ and it suffices to apply Lemma 3.1.8.

Remark. Let $(X, S)$ be the stratified toric variety arising from a semifan $\Omega \subset \Sigma$. The above correspondence between the cones of $\Omega$ and the strata of $S$ is order reversing in the sense that $\omega \preceq \omega^{\prime}$ iff $\operatorname{strat}\left(\omega^{\prime}\right)$ is contained in the closure of $\operatorname{strat}(\omega)$.

Lemma 3.1.10. Let $\Omega \subset \Sigma$ be an embedded $\Gamma$-semifan. Then all $\Gamma$-indecomposable faces of $\Sigma$ are in $\Omega$.

Proof. For a $\Gamma$-indecomposable face $\sigma$ write $\sigma=\omega(\sigma) \oplus^{\Gamma} \mathrm{r}(\sigma)$. This implies $\sigma=\omega(\sigma) \in \Omega$.

We conclude the section with some examples. The first two examples show that for any fan there are a maximal and a minimal embedded semifan.

Example 3.1.11. If $\Sigma$ is a fan in a lattice $N$, then $\Sigma \subset \Sigma$ is an embedded semifan. The corresponding stratification of the toric variety $X$ associated to $\Sigma$ is the decomposition of $X$ into the orbits of the big torus $T \subset X$. The orbit stratification is the finest among all toric stratifications of $X$.

Example 3.1.12. For a fan $\Sigma$ in a lattice $N$ and a toric action of $\Gamma$ on $X_{\Sigma}$ let $\operatorname{Sing}^{\Gamma}(\Sigma)$ denote the set of all maximal indecomposable parts $\operatorname{sing}^{\Gamma}(\sigma)$, where $\sigma \in \Sigma$. Then $\operatorname{Sing}^{\Gamma}(\Sigma) \subset \Sigma$ is an embedded $\Gamma$-semifan, and for any other embedded $\Gamma$-semifan $\Omega \subset \Sigma$ one has $\operatorname{Sing}^{\Gamma}(\Sigma) \subset \Omega$. The toric stratification corresponding to $\operatorname{Sing}^{\Gamma}(\Sigma) \subset \Sigma$ is the stratification by singularity type on the toric variety $X_{\Sigma}$ with the toric action of $\Gamma$. The toric stratification corresponding to $\operatorname{Sing}(\Sigma) \subset \Sigma$ is the coarsest among all toric stratifications of $X$. The toric stratification corresponding to $\operatorname{Sing}^{\Gamma}(\Sigma) \subset \Sigma$ is the coarsest among all toric stratifications of $X$ which are compatible with the action of $\Gamma$.

Example 3.1.13. Let $\mathbf{A}^{2} \supset K^{*} \times K^{*}$ be a toric variety with the strata $s_{0}:=\{(0,0)\}, s_{1}:=K^{*} \times\{0\}$, and $s_{2}:=\mathbf{A}^{2} \backslash\left(s_{0} \cup s_{1}\right)$. Then $\mathbf{A}^{2}$ with the above stratification is a stratified toric variety.

We have $\sigma_{0}=\langle(1,0),(0,1)\rangle, \sigma_{1}=\langle(0,1)\rangle, \sigma_{2}=\{(0,0)\}$,

$\left.\overline{\sigma_{0}}=\{\langle(1,0),(0,1)\rangle\}, \overline{\sigma_{1}}=\{\langle(0,1)\rangle\}, \overline{\sigma_{2}}=\{(0,0)\},\langle(1,0)\rangle\right\}$.

\section{Stratified toroidal VARIETIES}

4.1. Definition of a stratified toroidal variety.

Definition 4.1.1. (see also [20]). Let $X$ be a variety or a noetherian scheme over $K . X$ is called toroidal if for any $x \in X$ there exists an open affine neighborhood $U_{x}$ and an étale morphism $\phi_{x}: U_{x} \rightarrow X_{\sigma_{x}}$ into some affine toric variety $X_{\sigma_{x}}$.

Definition 4.1.2. Let $\Gamma$ act on noetherian schemes $X$ and $Y$. We say that a $\Gamma$-equivariant morphism $f: Y \rightarrow X$ is $\Gamma$-smooth (resp. $\Gamma$-étale) if there is a smooth (resp. étale) morphism $f^{\prime}: Y^{\prime} \rightarrow X^{\prime}$ of varieties with trivial action of $\Gamma$ and $\Gamma$-equivariant fiber square

$$
\begin{array}{rllll}
X \times X_{X^{\prime}} Y^{\prime} & \simeq & Y & \stackrel{f}{\rightarrow} & X \\
& \downarrow & & \downarrow \\
& Y^{\prime} & \stackrel{f^{\prime}}{\rightarrow} & X^{\prime}
\end{array}
$$

Lemma 4.1.3. Let $Y \rightarrow X$ be a $\Gamma$-smooth morphism. If the good quotient $X / / \Gamma$ exists then $Y / / \Gamma$ exists and $Y=Y / / \Gamma \times_{X / / \Gamma} X$. 
Proof. Let $Y_{0}:=Y^{\prime} \times_{X} X / / \Gamma$. Then $Y=Y_{0} \times_{X / / \Gamma} X$ and $Y / / \Gamma=Y_{0}$.

Definition 4.1.4. Let $X$ be a toroidal variety or a toroidal scheme. We say that an action of a group $\Gamma$ on $X$ is toroidal if for any $x \in X$ there exists an open $\Gamma$-invariant neighborhood $U$ and a $\Gamma$-equivariant, $\Gamma_{x}$-smooth, morphism $U \rightarrow X_{\sigma}$, into a toric variety $X_{\sigma}$ with a toric action of $\Gamma \supset \Gamma_{x}$.

Definition 4.1.5. Let $X$ be a toroidal variety or a toroidal scheme. We say that a stratification $S$ of $X$ is toroidal if any point $x$ in a stratum $s \in S$ admits an open neighborhood $U_{x}$ and an étale morphism $\phi_{x}: U_{x} \rightarrow X_{\sigma}$ into a stratified toric variety such that $s \cap U_{x}$ equals $\phi_{x}^{-1}\left(O_{\sigma}\right)$ and the intersections $s^{\prime} \cap U$, $s^{\prime} \in S$, are precisely the inverse images of strata in $X_{\sigma}$.

Definition 4.1.6. Let $X$ be toroidal variety or a toroidal scheme with a toroidal action of $\Gamma$. A toroidal stratification $S$ of $X$ is said to be compatible with the action of $\Gamma$ if

1. Strata of $S$ are invariant with respect to the action of $\Gamma$.

2. All points in one stratum $s$ have the same isotropy group $\Gamma_{s}$.

3. For any point $x \in s$ there exists a $\Gamma$-invariant neighborhood $U$ and a $\Gamma$-equivariant, $\Gamma_{s}$-smooth morphism $U \rightarrow X_{\sigma}$ into a $\Gamma$-stratified toric variety, such that $s \cap U_{x}$ equals $\phi_{x}^{-1}\left(O_{\sigma}\right)$ and the intersections $s^{\prime} \cap U, s^{\prime} \in S$, are precisely the inverse images of strata in $X_{\sigma}$.

If $X$ is a toroidal variety or a toroidal scheme with a toroidal stratification $S$ then we shall speak of a stratified toroidal variety (resp. a stratified toroidal scheme). If $X$ is a toroidal variety or a toroidal scheme with a toroidal action of $\Gamma$ and a toroidal stratification $S$ compatible with the action of $\Gamma$ then we shall speak of a $\Gamma$-stratified toroidal variety (resp. $\Gamma$-stratified toroidal scheme).

If $(X, S)$ is a $\Gamma$-stratified toroidal variety then for any stratum $s \in S$ set $\Gamma_{s}:=\Gamma_{x}$, where $x \in s$. Note that if $s \leq s^{\prime}$ then $\Gamma_{s} \subseteq \Gamma_{s^{\prime}}$.

Remark. A stratified toroidal variety can be considered as a $\Gamma$-stratified toroidal variety with trivial action of the trivial group $\Gamma=\{e\}$.

A simple generalization of Example 3.1 .12 is the following lemma:

Lemma 4.1.7. Let $X$ be a toroidal variety with a toroidal action of $\Gamma$. Let $\operatorname{Sing}^{\Gamma}(X)$ be the stratification determined by the singularity type $\operatorname{sing}^{\Gamma}(x)$ (see Definition 2.5.5). Then $\operatorname{Sing}^{\Gamma}(X)$ is a toroidal stratification compatible with the action of $\Gamma$.

Definition 4.1.8. (see also [39]). A toroidal embedding is a stratified toroidal variety such that any point $p$ in a stratum $s \in S$ admits an open neighborhood $U_{p}$ and an étale morphism of stratified varieties $\phi_{p}: U_{p} \rightarrow X_{\sigma}$ into a toric variety with orbit stratification (see Example 3.1.11).

A toroidal embedding with $\Gamma$-action is a $\Gamma$-stratified toroidal variety which is a toroidal embedding.

Remark. The definition of a toroidal embedding differs from the original definition in 39]. It is equivalent to the definition of a toroidal embedding without self-intersections (see Section 1.1).

\subsection{Existence of invariant stratifications on smooth varieties with $\Gamma$-action.}

Lemma 4.2.1. $\quad 1$. Let $X$ be a smooth variety with $\Gamma$-action. Then there exists a toroidal stratification $\operatorname{Sing}^{\Gamma}(X)$ of $X$ which is compatible with the action of $\Gamma$.

2. Let $X$ be a smooth variety with $\Gamma$-action and $D$ be a $\Gamma$-invariant divisor with simple normal crossings. Let $S_{D}$ be the stratification determined by the components of the divisor D. Set

$$
\operatorname{sing}^{\Gamma, D}(x):=\left(\operatorname{sing}^{\Gamma}(x), D(x)\right),
$$

where $D(x)$ is the set of components of $D$ passing through $x \in X$

Then $\operatorname{sing}^{\Gamma, D}$ determines a toroidal stratification $\operatorname{Sing}^{\Gamma}(X, D)$ compatible with the action of $\Gamma$. In particular all the closures of strata from $\operatorname{Sing}^{\Gamma}(X, D)$ have normal crossings with components of $D$.

Proof. For any $x$ we can find $\Gamma$-semiinvariant parameters $u_{1}, \ldots, u_{k}$ describing the orbit $\Gamma \cdot x$ in some $\Gamma$-invarinat neighborhood $U_{x}$ of $x$.

Additionally in (2) we require that each component of $D$ through $x \in X$ is described by one of the parameters in $U_{x}$. We define a smooth morphism $\phi_{x}: U_{x} \rightarrow \mathbf{A}^{k}$ by $\phi_{x}(y)=\left(u_{1}(y), \ldots, u_{k}(y)\right)$. The action of $\Gamma$ on $X$ defines the action of $\Gamma$ on $\mathbf{A}^{k}$ with standard coordinates be $\Gamma$-semiinvariant with corresponding 
weights. Then $\operatorname{Sing}^{\Gamma}\left(\mathbf{A}^{k}, D_{A}\right)$, where $D_{A}=\overline{\phi(D)}$, defines a $\Gamma$-invariant stratification on $\mathbf{A}^{k}$, whose strata are determined by standard coordinates. Therefore $\operatorname{Sing}^{\Gamma}(X, D)$ is also a stratification on $X$ which is locally a pull-back of $\operatorname{Sing}^{\Gamma}\left(\mathbf{A}^{k}, D_{A}\right)$.

The morphism $\phi_{x}$ is $\Gamma$-equivariant. Denote by $u_{1}, \ldots, u_{k}, \ldots, u_{n}$ all local $\Gamma_{x}$-semiinvariant parameters at $x$. Let $\widetilde{\phi}_{x}: U_{x} \rightarrow \mathbf{A}^{n}$ be the étale $\Gamma_{x}$-equivariant morphism defined by $\widetilde{\phi}_{x}(y):=\left(u_{1}(y), \ldots, u_{n}(y)\right)$. By Luna's fundamental lemma (see Luna [42]) we can find a $\Gamma_{x}$-invariant neighborhood $U_{x}^{\prime} \subset U_{x}$ for which the induced quotient morphism $\widetilde{\phi}_{x} / \Gamma_{x}: U_{x}^{\prime} / / \Gamma_{x} \rightarrow \mathbf{A}^{n} / / \Gamma_{x}$ is étale and $U_{x}^{\prime} \simeq U_{x}^{\prime} / / \Gamma_{x} \times_{\mathbf{A}^{n} / / \Gamma_{x}} \mathbf{A}^{n}$. By Sumihiro [67] we can find a $\Gamma$-invariant open affine $V \subset \Gamma \cdot U^{\prime}$ such that $V \simeq V / / \Gamma_{x} \times_{V / / \Gamma_{x}} \mathbf{A}^{n}$. Let $p: \mathbf{A}^{n} \rightarrow \mathbf{A}^{k}$ denote the standard projection on the first coordinates. Then $p \widetilde{\phi}_{x}: V \rightarrow \mathbf{A}^{k}$ is a $\Gamma$-equivariant $\Gamma_{x}$-smooth morphism. By definition $\left(X, \operatorname{Sing}^{\Gamma}(X, D)\right)$ is a $\Gamma$-stratified toroidal variety.

4.3. Conical semicomplexes. Here we generalize the notion of a semifan. For this we first have to figure out those semifans that describe affine stratified toric varieties. In analogy to usual cones and fans we shall denote them by small Greek letters $\sigma, \tau$, etc.:

Definition. Let $N$ be a lattice. A semicone in $N$ is a semifan $\sigma$ in $N$ such that the support $|\sigma|$ of $\sigma$ occurs as an element of $\sigma$.

The dimension of a semicone is the dimension of its support. Moreover, for an injection $\imath: N \rightarrow N^{\prime}$ of lattices, the image $\imath(\sigma)$ of a semicone $\sigma$ in $N$ is the semicone consisting of the images of all the elements of $\sigma$.

Note that every cone becomes a semicone by replacing it with the set of all its faces. Moreover, every semifan is a union of maximal semicones. Generalizing this observation, we build up semicomplexes from semicones:

Definition 4.3.1. Let $\Sigma$ be a finite collection of semicones $\sigma$ in lattices $N_{\sigma}$ with $\operatorname{dim}(\sigma)=\operatorname{dim}\left(N_{\sigma}\right)$. Moreover, suppose that there is a partial ordering " $\leq$ " on $\Sigma$.

We call $\Sigma$ a semicomplex if for any pair $\tau \leq \sigma$ in $\Sigma$ there is an associated linear injection $\imath_{\tau}^{\sigma}: N_{\tau} \rightarrow N_{\sigma}$ such that $\imath_{\tau}^{\sigma}\left(N_{\tau}\right) \subset N_{\sigma}$ is a saturated sublattice and

1. $\imath_{\tau}^{\sigma} \circ \imath_{\varrho}^{\tau}(\varrho)=\imath_{\varrho}^{\sigma}(\varrho)$,

2. $\imath_{\varrho}^{\sigma}(|\varrho|)=\imath_{\tau}^{\sigma}(|\tau|)$ implies $\varrho=\tau$,

3. $\sigma=\bigcup_{\tau \leq \sigma} \imath_{\tau}^{\sigma}(|\tau|)=\bigcup_{\tau \leq \sigma} \imath_{\tau}^{\sigma}(\tau)$.

As a special case of the above notion, we recover back the notion of a (conical) complex introduced by Kempf, Knudsen, Mumford and Saint-Donat:

Definition 4.3.2. A complex is a semicomplex $\Sigma$ such that every $\sigma \in \Sigma$ is a fan.

Definition 4.3.3. A semicomplex $\Sigma$ is called a $\Gamma$-semicomplex if there is a collection of algebraic groups $\Gamma_{\sigma} \subset T_{\sigma}$, where $\sigma \in \Sigma$, such that

1. Each $\sigma \in \Sigma$ is a $\Gamma_{\sigma}$-semicone.

2. For any $\tau \leq \sigma, \Gamma_{\tau} \subset \Gamma_{\sigma}$ and the morphism $\imath_{\tau}^{\sigma}$ induces a $\Gamma_{\tau}$-equivariant morphism $X_{\tau} \rightarrow X_{\sigma}$. Moreover $\Gamma_{\tau}=\left(\Gamma_{\sigma}\right)_{\tau}=\left\{g \in \Gamma_{\sigma} \mid g x=x, x \in O_{\tau}\right\}$.

Remarks. 1. Each semicomplex can be considered as a $\Gamma$-semicomplex with trivial groups $\Gamma_{\sigma}$

2. From now on we shall often identify vectors of $\sigma$ with their images under $\imath_{\tau}^{\sigma}$ if $\tau \leq \sigma$, and simplify the notation replacing $\imath_{\tau}^{\sigma}$ with set-theoretic inclusions.

3. In what follows we shall write $\sigma \preceq \sigma^{\prime}$ means that $\sigma$ is a face of the cone $\sigma^{\prime}$, while $\sigma \leq \sigma^{\prime}$ to mean that $\sigma$ is a face of the semicone $\sigma^{\prime}$.

4. In the case when $\Sigma$ is a conical complex the relations $\leq$ and $\preceq$ coincide.

5. Note that cones in a semicomplex intersect along a union of common faces.

Denote by $\operatorname{Aut}(\sigma)$ the group of automorphisms of the $\Gamma_{\sigma}$-semicone $\sigma$.

Lemma 4.3.4. For any $\Gamma$-semicomplex $\Sigma$ and any $\varrho \leq \tau \leq \sigma$ in $\Sigma$, there is an automorphism $\alpha_{\varrho} \in \operatorname{Aut}(\varrho)$ such that $\imath_{\tau}^{\sigma} \circ \imath_{\varrho}^{\tau}=\imath_{\varrho}^{\sigma} \alpha_{\varrho}$. 
Proof. By definition, the maps $\imath_{\tau}^{\sigma} \circ \imath_{\varrho}^{\tau}$ and $\imath_{\varrho}^{\sigma}$ are both linear isomorphisms of $\varrho$ onto the image $\imath_{\tau}^{\sigma} \circ \imath_{\varrho}^{\tau}(\varrho)=$ $\imath_{\varrho}^{\sigma}(\varrho)$. Thus $\alpha_{\varrho}:=\left(\imath_{\varrho}^{\sigma}\right)^{-1} \imath_{\tau}^{\sigma} \circ \imath_{\varrho}^{\tau}$.

Remarks. 1. It follows from Lemma 4.3 .4 that vectors in $\sigma$ are in general defined up to automorphisms from $\operatorname{Aut}(\sigma)$. Hence the notion of support of a semicomplex as a topological space which is the totality of such vectors is not well defined. However if we consider, for instance, vectors invariant under all such automorphisms then the relevant topological space can be constructed (see notion of stable support 6.1.1) and plays the key role for semicomplexes and their subdivisions.

2. In further considerations we shall introduce the notion of oriented semicomplex (see Definition 4.11.1) which allows a smaller group of automorphisms $\operatorname{Aut}(\sigma)^{0} \subset \operatorname{Aut}(\sigma)$. Consequently, the corresponding stable support of an oriented semicomplex is essentially larger. This allows one to perform more subdivisions and carry out certain important birational transformations.

Lemma 4.3.5. A $\Gamma$-semifan $\Sigma$ in $N$ determines a $\Gamma$-semicomplex $\Sigma^{\text {semic }}$ where each cone $\sigma \in \Sigma$ determines the semicone consisting of all faces $\tau \leq \sigma$ and $\Gamma_{\tau}=\left\{g \in \Gamma \mid g x=x, x \in O_{\tau}\right\}$.

Definition 4.3.6. By an isomorphism of two $\Gamma$-semicomplexes $\Sigma \rightarrow \Sigma^{\prime}$ we mean a face bijection $\Sigma \ni \sigma \mapsto$ $\sigma^{\prime} \in \Sigma^{\prime}$ such that $\Gamma_{\sigma}=\Gamma_{\sigma^{\prime}}$, along with a collection of face $\Gamma_{\sigma^{-}}$-isomorphisms $j_{\sigma}: \sigma \rightarrow \sigma^{\prime}$, such that for any $\tau \leq \sigma$, there is a $\Gamma_{\tau}$-automorphism $\beta_{\tau}$ of $\tau$ such that $j_{\sigma} \imath_{\tau}^{\sigma}=\imath_{\tau^{\prime}}^{\sigma^{\prime}} j_{\tau} \beta_{\tau}$.

\subsection{Inverse systems of affine algebraic groups.}

Definition 4.4.1. By an affine proalgebraic group we mean an affine group scheme that is the limit of an inverse system $\left(G_{i}\right)_{i \in \mathbf{N}}$ of affine algebraic groups and algebraic group homomorphisms $\phi_{i j}: G_{i} \rightarrow G_{j}$, for $j \geq i$.

Lemma 4.4.2. Consider the natural morphism $\phi_{i}: G \rightarrow G_{i}$. Then $H_{i}:=\phi_{i}(G)$ is an algebraic subgroup of $G_{i}$, all induced morphisms $H_{j} \rightarrow H_{i}$ for $i \leq j$ are epimorphisms and $G=\lim _{\leftarrow} G_{i}=\lim _{\leftarrow} H_{i}$. In particular $K\left[H_{i}\right] \subset K\left[H_{i+1}\right]$ and $K[G]=\bigcup K\left[H_{i}\right]$.

Proof. The set $H_{i}^{\prime}:=\bigcap_{j>i} \phi_{j i}\left(G_{j}\right)$ is an intersection of algebraic subgroups of $G_{i}$. Hence it is an algebraic subgroup of $G_{i}$. Note that the induced homomorphisms $\phi_{i j}^{H}: H_{j}^{\prime} \rightarrow H_{i}^{\prime}$ are epimorphisms and that $G=$ $\lim _{\leftarrow} G_{i}=\lim _{\leftarrow} H_{i}^{\prime}$. Consequently, $\phi_{i}(G)=H_{i}=H_{i}^{\prime}$.

Lemma 4.4.3. The set $G^{K}$ of $K$-rational points of $G$ is an abstract group which is the inverse limit $G^{K}=$ $\lim _{\leftarrow} G_{i}^{K}$ in the category of abstract groups.

Proof. By the previous lemma we can assume all morphisms $G_{i} \rightarrow G_{j}$ to be epimorphisms. Any $K$ rational point $x$ in $G$ is mapped to $K$-rational points $x_{i}$ in $G_{i}$. This gives an abstract group homomorphism $\phi: G^{K} \rightarrow \lim _{\leftarrow} G_{i}^{K}$. We also have $K[G]=\bigcup K\left[G_{i}\right]$. We have to show that any point $x$ of $\lim _{\leftarrow} G_{i}^{K}$ determines a unique point $y$ in $G^{K}$. The point $x$ determines a sequence of maximal ideals $m_{i} \in K\left[G_{i}\right]$ such that $m_{i} \subset m_{i+1}$. Let $m:=\bigcup m_{i}$. Then $m \cap K\left[G_{i}\right]=m_{i}$. Let $f \in K[G]$. Then $f \in K\left[G_{i}\right]$ for some $i$ and $f-k \in m_{i}$ for some $k \in K$. Hence $f \in k+m$, which means that $K[G] / m=K$. The ideal $m$ defines a $K$-rational point $y$ on $G^{K}$ which is mapped to $x$. Note that the point $y$ is unique since there is a unique ideal $m$ for which $m \cap K\left[G_{i}\right]=m_{i}$.

By abuse of notation we shall identify $G$ with $G^{K}$.

4.5. Group of automophisms of the completion of a local ring. Let $X$ be a stratified noetherian scheme over $\operatorname{Spec}(K)$. Let $S$ denote the stratification and $x \in X$ be a closed $K$-rational point. Let $\widehat{\mathcal{O}}_{X, x}$ denote the completion of the local ring of $x$ on the scheme $X$. Let $u_{1}, \ldots u_{k} \in \widehat{\mathcal{O}}_{X, x}$ generate the maximal ideal $m_{x, X} \subset \widehat{\mathcal{O}}_{X, x}$. Then $\widehat{\mathcal{O}}_{X, x}=K\left[\left[u_{1}, \ldots, u_{k}\right]\right] / I$, where $I$ is the defining ideal. Set $\widehat{X}_{x}:=\operatorname{Spec}\left(\widehat{\mathcal{O}}_{X, x}\right)$ and $X_{x}^{(n)}:=\operatorname{Spec}\left(\mathcal{O}_{X, x} / m_{x, X}^{n+1}\right)$ for $n \in \mathbf{N}$.

Let $\operatorname{Aut}\left(\widehat{X}_{x}, S\right)$ (respectively $\operatorname{Aut}\left(X_{x}^{(n)}, S\right)$ ) denote the automorphism group of $\widehat{X}_{x}$ (resp. $\left.X_{x}^{(n)}\right)$ preserving all closures $\bar{s} \ni x$ of strata $s \in S$. (resp. preserving all subschemes

$\operatorname{Spec}\left(\mathcal{O}_{X, x} /\left(I_{\bar{s}}+m_{x, X}^{n+1}\right)\right)$, for all $\left.s \in S\right)$ 
If $\Gamma$ acts on $X$ denote by $\operatorname{Aut}^{\Gamma}\left(\widehat{X}_{x}, S\right)$ (respectively $\operatorname{Aut}^{\Gamma}\left(X_{x}^{(n)}, S\right)$ ) the group of all $\Gamma_{x}$-equivariant atomorphisms of $\left(\widehat{X}_{x}, S\right)$ (resp. $\left.\left(X_{x}^{(n)}, S\right)\right)$.

In further considerations set $G:=\operatorname{Aut}\left(\widehat{X}_{x}, S\right)\left(\right.$ resp. $\left.G:=\operatorname{Aut}^{\Gamma}\left(\widehat{X}_{x}, S\right)\right)$ in the case of the action of $\Gamma$, and $G_{n}:=\operatorname{Aut}\left(X_{x}^{(n)}, S\right)\left(\right.$ resp. $\left.G_{n}:=\operatorname{Aut}^{\Gamma}\left(X_{x}^{(n)}, S\right)\right)$.

Lemma 4.5.1. $\quad 1 . G_{n}$ is an algebraic group acting on $X_{x}^{(n)}$. That is there exists a co-action

$$
\Phi_{n}^{*}: \mathcal{O}_{X, x} / m_{x, X}^{n+1} \rightarrow K\left[G_{n}\right] \otimes \mathcal{O}_{X, x} / m_{x, X}^{n+1}
$$

defining the action morphism $\Phi_{n}: G_{n} \times X^{(n)} \rightarrow X^{(n)}$

and an action automorphism $\Psi_{n}:=\pi_{n} \times \Phi_{n}: G_{n} \times X^{(n)} \rightarrow G_{n} \times X^{(n)}$

where $\pi_{n}$ is the projection on $G_{n}$.

2. The $G_{n}$ form an inverse system of algebraic groups and $G=\lim _{\leftarrow} G_{n}$ is a proalgebraic group.

3. The co-actions in (1) define a ring homomorphism $\Phi^{*}: \widehat{\mathcal{O}}_{X, x} \rightarrow K[G]\left[\left[\widehat{\mathcal{O}}_{x, X}\right]\right]$

and a formal action morphism $\Phi: G \widehat{\times} \widehat{X}_{x}:=\operatorname{Spec} K[G]\left[\left[\widehat{\mathcal{O}}_{X, x}\right]\right] \rightarrow \widehat{X}_{x}$

such that for any $g \in G^{K}$ the restriction $\Phi_{\mid\{g\}} \times \widehat{X}_{x}:\{g\} \widehat{\times} \widehat{X}_{x} \rightarrow \widehat{X}_{x}$ is given by the action of $g$.

4. The automorphisms $\Psi_{n}$ of $X^{(n)}$ define a formal action automorphism $\Psi$ of

such that for any $g \in G^{K}$ the restriction $\Psi_{\mid\{g\} \widehat{\times} \widehat{X}_{x}}:\{g\} \widehat{\times} \widehat{X}_{x} \rightarrow\{g\} \widehat{\times} \widehat{X}_{x}$ is the automorphism given by the action of $g$.

5. Let $u_{1}, \ldots, u_{k}$ denote the local parameters on $\widehat{X}_{x}$. There exist regular functions $g_{\alpha \beta}$ generating $K[G]$ such that the action is given by $u^{\alpha} \mapsto \sum g_{\alpha \beta} u^{\beta}$, where $\alpha, \beta \in \mathbb{Z}_{\geq 0}^{n}$ are multiindices.

Proof. (1) Write $K\left[X_{x}^{(n)}\right]=K\left[u_{1}, \ldots, u_{k}\right] /\left(I+m_{x}^{n+1}\right)$. Set $W^{n}:=K\left[u_{1}, \ldots, u_{k}\right] /\left(m_{x}^{n+1}\right)$, $I^{n}:=\left(I+m_{x}^{n+1}\right) / m_{x}^{n+1}, I_{s}^{n}:=\left(I_{s}+m_{x}^{n+1}\right) / m_{x}^{n+1}$.

Define the product $W^{n} \times W^{n} \rightarrow W^{n}$ to be the bilinear map for which $u^{\alpha_{1}} \cdot u^{\alpha_{2}}=u^{\alpha_{1}+\alpha_{2}}$. Set

$$
H_{n}:=\left\{g \in \mathrm{Gl}\left(W^{n}\right) \mid g\left(u^{\alpha_{1}} \cdot u^{\alpha_{2}}\right)=g\left(u^{\alpha_{1}}\right) \cdot g\left(u^{\alpha_{2}}\right)\right\} .
$$

$H_{n}$ is an algebraic subgroup of $\mathrm{Gl}\left(W^{n}\right)$. Then

$$
G_{n}=\left\{g \in H^{n} \mid g\left(I^{n}\right)=I^{n}, g\left(I_{s}^{n}\right)=I_{s}^{n}, \quad \text { for all } \quad s \in S, g a=a g \quad \text { for all } \quad a \in \Gamma_{x}\right\}
$$

is algebraic. The embedding $G_{n} \subset \operatorname{Gl}\left(W^{n}\right)$ determines the natural co-action $K\left[W^{n}\right] \rightarrow K\left[G_{n}\right] \otimes K\left[W_{n}\right]$,

$$
u^{\alpha} \mapsto \sum_{\beta \in \mathbf{Z}_{\geq 0}^{n}} g_{\alpha \beta} u^{\beta},
$$

which factors to $K\left[X_{x}^{(n)}\right] \rightarrow K\left[G_{n}\right] \otimes K\left[X_{x}^{(n)}\right]$.

(2) If the morphism $X_{x}^{(n)} \hookrightarrow X_{x}^{(n+1)}$ commutes with automorphisms, i.e. if $\Phi$ is an automorphism of $X_{x}^{(n+1)}$, then $\Phi_{\mid X^{(n)}}: X^{(n)} \rightarrow X^{(n)} \subset X_{x}^{(n+1)}$ is an automorphism of $X^{(n)}$. This defines the morphisms $\operatorname{Aut}\left(X_{x}^{n+1}, S\right) \rightarrow \operatorname{Aut}\left(X_{x}^{(n)}, S\right)$.

The $K$-rational points of the proalgebraic group $G=\lim _{\leftarrow} G_{n}$ can be identified, by Lemma 4.4.3, with ( $\Gamma_{x}$-equivariant) automorphisms of $\widehat{X}_{x}$ preserving strata.

(3) The morphisms $\widehat{\mathcal{O}}_{X, x} \rightarrow K\left[G_{n}\right]\left[\left[\mathcal{O}_{x, X}\right]\right] / m_{x, X}^{n} \rightarrow K[G]\left[\left[\widehat{\mathcal{O}}_{X, x}\right]\right] / m_{x, X}^{n}$ determine

$$
\widehat{\mathcal{O}}_{X, x} \rightarrow \lim _{\leftarrow} K[G]\left[\left[\widehat{\mathcal{O}}_{X, x}\right]\right] / m_{x, X}^{n}
$$

(4) and (5) follow from (1)

Lemma 4.5.2. Let $G=\lim _{\leftarrow} G_{i}$ be a connected proalgebraic group acting on $\widehat{X}_{x}$. Let $\Phi: G \widehat{\times} \widehat{X}_{x} \rightarrow \widehat{X}_{x}$ be the action morphism and $\pi: G \widehat{\times} \widehat{X}_{x} \rightarrow \widehat{X}_{x}$ be the standard projection. Then for any ideal I on $\widehat{X}_{x}$ the following conditions are equivalent:

- I is invariant with respect to the action of the abstract group $G^{K}$

- $\Phi^{*}(I)=\pi^{*}(I)$

Proof. It suffices to prove the equivalence of the conditions for any scheme $X_{x}^{(n)}$ and ideal $I_{n}=I \cdot K\left[X_{x}^{(n)}\right]$. In this situation the assertion reduces to the well known case of an algebraic action of $G_{n}$ on $X_{x}^{(n)}$. If 
$\Phi^{*}\left(I_{n}\right)=\pi^{*}(I)$ then for any $g \in G_{n}^{K}$ and $f \in I_{n}$, we have $g \cdot f=\Phi_{g}^{*}(f) \in \pi_{g}^{*}\left(I_{n}\right)=I_{n}$. Thus $I_{n}$ is $G_{n}^{K}$-invariant.

Now suppose $I_{n}$ is $G_{n}^{K}$-invariant. Let $\Phi^{*}(f)=\sum f_{i} g_{i}$ for $f \in I_{n}$. We can assume that $g_{i} \in K\left[G_{n}\right]$ are linearly independent. Hence we can find elements $g^{i} \in G_{n}^{K}$ such that $v_{i}:=\left(g_{i}\left(g^{1}\right), \ldots, g_{i}\left(g^{l}\right)\right)$ are linearly independent vectors for $i=1, \ldots, l$. Then we find coefficients $\alpha_{i j}$ such that $\sum_{j} \alpha_{i j} v_{j}=\left(0, \ldots, 1_{j}, \ldots, 0\right)$. Then $f_{i}=\sum_{j} \alpha_{i j} f_{i} g_{i}\left(g^{j}\right)=\sum_{j} \alpha_{i j}\left(g^{j} \cdot f_{i}\right) \in I_{n}$. Thus for any $f \in I_{n}, \Phi^{*}(f) \in \pi^{*}\left(I_{n}\right)$, which gives $\Phi^{*}(I) \subset \pi^{*}(I)$.

Denote by inv the automorphism of $G \widehat{\times} \widehat{X}_{x}$ induced by taking the inverse $g \mapsto g^{-1}$. Then $\pi^{*}(I)=$ $\Psi^{-1 *} \Phi^{*}(I) \subset \Psi^{-1 *} \pi^{*}(I)=\Psi^{*} \operatorname{inv}^{*} \pi^{*}(I)=\Psi^{*} \pi^{*}(I)=\Phi^{*}(I)$.

Let $L$ denote an algebraically closed field containing $K$. Set

$$
\begin{gathered}
X_{x}^{(n) L}:=X_{x}^{(n)} \times_{\operatorname{Spec} K} \operatorname{Spec} L, \\
\widehat{X}_{x}^{L}:=\lim _{\leftarrow} X_{x}^{(n) L}=\operatorname{Spec} L\left[\left[\mathcal{O}_{X, x}\right]\right]
\end{gathered}
$$

Let $\operatorname{Aut}_{L}^{\Gamma}\left(X_{x}^{(n) L}, S\right)$ denote the group of $\Gamma_{x}$-equivariant $L$-automorphisms of $X_{x}^{(n) L}$ preserving the relevant ideals of strata and $G:=\operatorname{Aut}_{L}\left(\widehat{X}_{x}^{L}, S\right)$ denote the group of $L$-automorphisms of $\widehat{X}_{x}^{L}$.

Lemma 4.5.3. 1. $\left.\operatorname{Aut}_{L}^{\Gamma}\left(X_{x}^{(n) L}, S\right)=\operatorname{Aut}_{K}^{\Gamma}\left(X_{x}^{(n)}, S\right)\right) \times$ Spec $K \operatorname{Spec} L$.

2. $\operatorname{Aut}_{L}^{\Gamma}\left(\widehat{X}_{x}^{L}, S\right)=\operatorname{Aut}_{K}^{\Gamma}\left(\widehat{X}_{x}, S\right) \times_{\text {Spec } K} \operatorname{Spec} L$.

3. Let $G \subset \operatorname{Aut}_{K}\left(\widehat{X}_{x}, S\right)$ be a proalgebraic subgroup. An ideal $I \subset \widehat{\mathcal{O}}_{X, x}$ is $G$-invariant iff $I \cdot L\left[\left[\mathcal{O}_{X, x}\right]\right]$ is $G \times \times_{\operatorname{Spec} K} \operatorname{Spec} L$-invariant.

Proof. (1) and (2) follow from the construction of $\operatorname{Aut}_{K}^{\Gamma}\left(\widehat{X}_{x}, S\right)$.

$(3)(\Rightarrow)$ It follows from Lemma 4.5 .2 that $I \subset \widehat{\mathcal{O}}_{X, x}$ is $G$-invariant iff $\Phi^{*}(I)=\pi^{*}(I)$ or $\Psi^{*}\left(\frac{I+m_{x}^{n}}{m_{x}^{n}}\right)=$ $\pi^{*}\left(\frac{I+m_{x}^{n}}{m_{x}^{n}}\right)$. Let $\Psi^{L}$ and $\pi$ be pull-backs of the morphisms $\Psi$ and $\pi^{L}$ under $\operatorname{Spec} L \rightarrow \operatorname{Spec} K$. Then $\Psi^{L *}\left(\frac{I+m_{x}^{n}}{m_{x}^{n}} \cdot L\right)=\pi^{L *}\left(\frac{I+m_{x}^{n}}{m_{x}^{n}} \cdot L\right) \subset L \otimes_{K} \widehat{\mathcal{O}}_{X, x} / m_{x}^{n}$, which shows, by Lemma 4.5.2, that $I \cdot L\left[\left[\mathcal{O}_{X, x}\right]\right]$ is $G \times \operatorname{Spec} K \operatorname{Spec} L$-invariant.

$(\Leftarrow)$. Assume the latter holds then $\Psi^{L *}\left(\frac{I+m_{x}^{n}}{m_{x}^{n}} \cdot L\right)=\pi^{L *}\left(\frac{I+m_{x}^{n}}{m_{x}^{n}} \cdot L\right) \subset L \otimes_{K} \widehat{\mathcal{O}}_{X, x} / m_{x}^{n}$. Let $\operatorname{Gal}(L / K)$ be the Galois group of the extension $K \subset L$. Both homomorphisms $\pi^{L *}$ and $\Psi^{L *}$ are $\operatorname{Gal}(L / K)$-equivariant. Considering $\operatorname{Gal}(L / K)$-invariant elements gives $\Psi^{*}\left(\frac{I+m_{x}^{n}}{m_{x}^{n}}\right)=\pi^{*}\left(\frac{I+m_{x}^{n}}{m_{x}^{n}}\right)$.

Example 4.5.4. Let $\phi_{n}: \operatorname{Aut}\left(\widehat{X}_{x}, S\right) \rightarrow \operatorname{Aut}\left(X_{x}^{(n)}, S\right)$ denote the natural morphisms. For $n=1$ we get the differential mapping:

$$
\left.d=\phi_{1}: \operatorname{Aut}\left(\widehat{X}_{x}, S\right) \longrightarrow \operatorname{Aut}\left(X_{x}^{(1)}, S\right) \subset \operatorname{Gl}\left(\operatorname{Tan}_{X, x}\right)\right) .
$$

4.6. Proof of Demushkin's Theorem. In what follows we shall use the following generalization of Theorem 2.5.1.

Lemma 4.6.1. Let $\sigma$ and $\tau$ be two $\Gamma$-semicones in isomorphic lattices $N_{\sigma} \simeq N_{\tau}$.

Then there exists a $\Gamma$-equivariant isomorphism $\widehat{X}_{\sigma} \simeq \widehat{X}_{\tau}$ preserving strata iff there exists an isomorphism of $\Gamma$-semicones $\sigma \simeq \tau$.

For a $\Gamma$-semicone $\sigma$ in a lattice $N_{\sigma}$ denote by $\operatorname{Aut}\left(\widehat{X}_{\sigma}\right)$ the group of all $\Gamma$-equivariant automorphisms of $\widehat{X}_{\sigma}$ which preserve strata defined by faces of the $\Gamma$-semicone $\sigma$.

Lemma 4.6.2. (Demushkin [22], also Gubeladze [27]) Let $\sigma$ and $\tau$ be two $\Gamma$-semicones in isomorphic lattices $N_{\sigma} \simeq N_{\tau}$.

1. The torus $T_{\sigma}$ associated to $\widehat{X}_{\sigma}$ determines a maximal torus in the proalgebraic group $G:=\operatorname{Aut}\left(\widehat{X}_{\sigma}\right)$.

2. Let $d: G \rightarrow \mathrm{Gl}\left(\operatorname{Tan}_{X_{\sigma}, O_{\sigma}}\right)$ be the differential morphism as in Example 4.5.4. Then $d\left(T_{\sigma}\right)$ is a maximal torus in $d(G)$.

3. Any $\Gamma$-equivariant isomorphism $\widehat{\phi}: \widehat{X}_{\sigma} \simeq \widehat{X}_{\tau}$, preserving strata determines an action of the torus $T_{\tau}$ on $\widehat{X}_{\sigma}$ and a group embedding $T_{\tau} \hookrightarrow G$. If $d\left(T_{\sigma}\right)=d\left(T_{\tau}\right)$ then $T_{\sigma}$ and $T_{\tau}$ are conjugate in $G$.

4. Any two tori $T_{\sigma}, T_{\tau} \subset G$ which are determined by $\widehat{\phi}$, are conjugate in $G$. 
Proof. (1) The torus $T_{\sigma}$ is maximal in $G$ since its centralizer consists of $T_{\sigma}$-equivariant automorphisms of $\widehat{X}_{\sigma}$, preserving strata and therefore it coincides with $T_{\sigma}\left(T_{\sigma}\right.$-equivariant automorphisms multiply characters by constants and are defined by elements of $T_{\sigma}$ ); (see also Step 4 in 22]).

(2) By Lemma 4.4 .2 we can write $G=\lim _{\leftarrow} G_{i}$, where all homomorphisms $G_{i} \rightarrow G_{j}$ are epimorphisms. We show that the relevant images of $T_{\sigma}$ are maximal tori in all groups $G_{i}$. Otherwise we find maximal tori $T_{i} \subset G_{i}$ containing the images of $T_{\sigma}$, such that $\lim _{\leftarrow} T_{i}$ is a maximal torus in $G$ containing $T_{\sigma}$ as a proper subgroup (see [10] Proposition 11.14(1)); (see also step 9 in [22]).

(3) Let $T_{\sigma} \subset G$ be the maximal torus associated to $\widehat{X}_{\sigma}$. Let $u_{1}, \ldots, u_{k}$ be characters of $T_{\sigma}$ generating the maximal ideal of $O_{\sigma}$ and $x_{1}, \ldots, x_{k}$ be semiinvariant functions determined by the action of $T_{\sigma}$, induced on the tangent space $\operatorname{Tan}_{O_{\sigma}, X_{\sigma}}$. Characters of $T_{\sigma}$ define the natural $T_{\sigma}$-equivariant embedding $\phi_{\sigma}: \widehat{X}_{\sigma} \rightarrow \operatorname{Tan}_{O_{\sigma}, X_{\sigma}}$ into the tangent space induced by the ring epimorphism $\phi_{\sigma}^{*}: K\left[\left[x_{1}, \ldots, x_{k}\right]\right] \rightarrow K\left[\left[u_{1}, \ldots, u_{k}\right]\right]$ sending $x_{i}$ to $u_{i}$. The morphism $\phi_{\sigma}$ determines the isomorphism $\widehat{\phi}_{\sigma}: \widehat{X}_{\sigma} \rightarrow \widehat{\phi}_{\sigma}\left(\widehat{X}_{\sigma}\right)$ preserving strata corresponding to the isomorphism $\widehat{\phi}_{\sigma}^{*}: K\left[\left[x_{1}, \ldots, x_{k}\right]\right] / I \rightarrow K\left[\left[u_{1}, \ldots, u_{k}\right]\right]$ where the ideal $I$ is generated by all elements $x^{\alpha}-x^{\beta}$ where $x^{\alpha}, x^{\beta}$ are monomials on which $T_{\sigma}$ acts with the same weights.

If $d\left(T_{\sigma}\right)=d\left(T_{\tau}\right)$ then $\phi_{\sigma}\left(\widehat{X}_{\sigma}\right)=\phi_{\tau}\left(\widehat{X}_{\tau}\right)=\operatorname{Spec} K\left[\left[x_{1}, \ldots, x_{k}\right]\right] / I$. Consequently, $\beta:=\phi_{T_{\tau}}^{-1} \phi_{T_{\sigma}}$ is a $T_{\sigma}=d\left(T_{\sigma}\right)$-equivariant isomorphism $\widehat{X}_{\sigma} \rightarrow \widehat{X}_{\tau}$ preserving strata. It follows that $\beta$ determines a conjugating automorphism with $T_{\sigma}=\beta T_{\tau} \beta^{-1}$.

(4) By Borel's theorem $(10])$ and (2), the tori $d\left(T_{\sigma}\right)$ and $d\left(T_{\tau}\right)$ are conjugate in $d(G)$. Consequently, we can find a torus $T$ conjugate to $T_{\sigma}$ and such that $d\left(T_{\tau}\right)=d(T)$. By (3) $T_{\tau}$ and $T$ are conjugate.

Proof of Lemma 4.6.1. Let $\widehat{\phi}: \widehat{X}_{\sigma} \rightarrow \widehat{X}_{\tau}$ be a $\Gamma$-equivariant isomorphism preserving strata. By Lemma 4.6 .2 there is a $\Gamma$-equivariant automorphism $\alpha$ of $\widehat{X}_{\sigma}$ for which $T_{\sigma}=\alpha T_{\tau} \alpha^{-1}$. Then $T_{\sigma}$ acts on $\widehat{X}_{\tau}$ as a big torus and the $\Gamma$-equivariant isomorphism $\hat{\phi} \circ \alpha^{-1}: \widehat{X}_{\sigma} \rightarrow \widehat{X}_{\tau}$ is $T_{\sigma}$-equivariant and preserves strata. Such an isomorphism yields an isomorphism of the associated $\Gamma$-semicones.

4.7. Singularity type of nonclosed points. For a ring $L$ and a cone $\sigma$ in $N_{\sigma}$ denote by $L\left[\left[\sigma^{\vee}\right]\right]$ the completion of the ring $L\left[\sigma^{\vee}\right]$ at the ideal $m_{\sigma}$ of $O_{\sigma}$.

Lemma 4.7.1. Let $L$ be a ring containing $K$, and $\sigma$ and $\tau$ be $\Gamma$-semicones in isomorphic lattices $N_{\sigma} \simeq N_{\tau}$. Let $m_{\sigma}, m_{\tau}$ denote the ideals of $O_{\sigma}$ and $O_{\tau}$ respectively. The action of $\Gamma$ on $K\left[\left[\sigma^{\vee}\right]\right]$ and $K\left[\left[\tau^{\vee}\right]\right]$ induces an action on $L\left[\left[\sigma^{\vee}\right]\right]$ and $L\left[\left[\tau^{\vee}\right]\right]$ which is trivial on $L$.

Any $\Gamma$-equivariant isomorphism $\phi: L\left[\left[\sigma^{\vee}\right]\right] \simeq L\left[\left[\tau^{\vee}\right]\right]$ for which $\phi\left(m_{\sigma}\right)=m_{\tau}$ can be decomposed as $\phi=\phi_{0} \phi_{1}$, where $\phi_{0}$ is a $\Gamma$-equivariant automorphism of $L\left[\left[\tau^{\vee}\right]\right]$ identical on monomials and $\phi_{1}$ is a $\Gamma$ equivariant L-isomorphism.

Proof. (1) The restriction of $\phi$ to $L$ is a ring monomorphism $\phi_{\mid L}: L \rightarrow L\left[\left[\tau^{\vee}\right]\right]$. This ring homomorphism can be extended to a ring homomorphism $\phi_{0}: L\left[\left[\tau^{\vee}\right]\right] \rightarrow L\left[\left[\tau^{\vee}\right]\right]$ by sending monomials identically to the same monomials, i.e. $\phi_{0}\left(\sum_{\alpha \in \tau^{\vee}} a_{\alpha} x^{\alpha}\right)=\sum_{\alpha \in \tau^{\vee}} \phi_{0}\left(a_{\alpha}\right) x^{\alpha}$, where $\phi_{0}\left(a_{\alpha}\right)=\sum_{\beta \in \tau^{\vee}} a_{\alpha \beta} x^{\beta}$. Note that in the expression $\sum_{\alpha \in \tau^{\vee}} \phi_{0}\left(a_{\alpha}\right) x^{\alpha}$, the coefficient of $x^{\alpha}$ is a finite sum since there are finitely many possibilities to express $\alpha$ as $\alpha^{\prime}+\beta$ where $\alpha^{\prime}, \beta \in \tau^{\vee}$. Thus the above expression defines a formal power series and consequently $\phi_{0}$ is a ring homomorphism. Then $\phi_{0 \mid L}$ determines an automorphism $\phi_{1}: L \rightarrow L\left[\left[\tau^{\vee}\right]\right] / m_{\tau}=L$. Let $\phi_{2}$ denote the automomorphism of $L\left[\left[\tau^{\vee}\right]\right]$ induced by the $\phi_{1}$ and identical on monomials. The composition $\psi=\phi_{2}^{-1} \phi_{0}$ is a ring endomorphism which as a $K$-linear transformation can be written in the form $\psi=i d+p$, where $p\left(m^{i}\right) \subset m^{i+1}$. Such a linear transformation is invertible (with inverse $i d-p+p^{2}-\ldots$ ), hence $\psi$ is a linear isomorphism and ring isomorphism. This implies that $\phi_{0}$ is an automorphism and $\phi_{1}:=\phi_{0}^{-1} \phi$ is an $L$-isomorphism.

Note that the above morphisms are $\Gamma$-equivariant.

The following is a generalization of the Demushkin Lemma.

Lemma 4.7.2. Let $\sigma$ and $\tau$ be $\Gamma$-semicones in isomorphic lattices $N_{\sigma} \simeq N_{\tau}$, and $L$ be a field containing $K$. Consider the induced action of $\Gamma$ on $L\left[\left[\sigma^{\vee}\right]\right]$ and $L\left[\left[\tau^{\vee}\right]\right]$ which is trivial on L. There exists a $\Gamma$-equivariant isomorphism $L\left[\left[\sigma^{\vee}\right]\right] \simeq L\left[\left[\tau^{\vee}\right]\right]$ over $K$, preserving strata iff $\sigma$ and $\tau$ are $\Gamma$-isomorphic. 
Proof. Denote by $\phi$ an isomorphism $L\left[\left[\sigma^{\vee}\right]\right] \stackrel{\phi}{\simeq} L\left[\left[\tau^{\vee}\right]\right]$. By Lemma 4.7.1, $\phi=\phi_{0} \phi_{1}$, where $\phi_{1}$ is an $L$-isomorphism. Tensoring with the algebraic closure $\bar{L}$ of $L$ and taking completion determines an $\bar{L}$ isomorphism $\bar{\phi}_{1}: \bar{L}\left[\left[\sigma^{\vee}\right]\right] \simeq \bar{L}\left[\left[\tau^{\vee}\right]\right]$. It suffices to apply Theorem 2.5.4 to the above $\bar{L}$-isomorphism

Note that the above isomorphisms are $\Gamma$-equivariant.

This lemma allows us to extend the notion of singularity type to any nonclosed points on toric varieties $X_{\Delta}$ and even on some other toroidal schemes.

Lemma 4.7.3. Let $\Delta$ be a subdivision of $\sigma$ and $\widehat{X}_{\Delta}:=X_{\Delta} \times_{X_{\sigma}} \widehat{X}_{\sigma}$ be a toroidal scheme with an action of a group $\Gamma \subset T_{\sigma}$. Let $p \in Y=\widehat{X}_{\Delta}$ be a point which need not be closed. Set $\Gamma_{p}:=\left\{g \in \Gamma \mid g(p)=p, g_{\mid K_{p}}=\right.$ $\left.\operatorname{id}_{\mid K_{p}}\right\}$.

1. Let $O_{\tau, Y} \in Y$ denote the locally closed subscheme defined by a toric orbit $O_{\tau} \subset X_{\Delta}$, where $\tau \in \Delta$. Then $\widehat{\mathcal{O}}_{O_{\tau}, Y} \simeq K_{O_{\tau}}\left[\left[\tau^{\vee}\right]\right]$, where $K_{O_{\tau}}$ is the residue field of $O_{\tau}$.

2. There is a $\Gamma_{p}$-equivariant isomorphism $\widehat{\mathcal{O}}_{p, Y} \simeq K_{p}\left[\left[\sigma_{p}^{\vee}\right]\right]$, where $K_{p}$ is the residue field of $p$ and $\sigma_{p}$ is a uniquely determined cone. Moreover $\operatorname{sing}^{\Gamma}(p):=\left(\Gamma_{p}, \operatorname{sing}^{\Gamma_{p}}\left(\sigma_{p}\right)\right)=\operatorname{sing}^{\Gamma}(\tau):=\left(\Gamma_{\tau}, \operatorname{sing}^{\Gamma_{\tau}}(\tau)\right)$, where $O_{\tau, Y} \in Y$ is the minimal orbit scheme containing $p$.

Proof. (1) Let $X_{\tau, Y}:=X_{\tau} \times_{X_{\sigma}} \widehat{X_{\sigma}} \subset Y$. Then $K\left[X_{\tau, Y}\right]=K\left[\tau^{\vee}\right] \otimes_{K\left[\sigma^{\vee}\right]} K\left[\left[\sigma^{\vee}\right]\right]$. Let $T_{\tau} \subset T$ be the torus corresponding to the sublattice $N_{\tau}:=N \cap \operatorname{lin}(\sigma)$. Then $T_{\tau}$ acts on $K\left[X_{\tau, Y}\right]$ with nonnegative weights. Therefore the subring of $T_{\tau^{-}}$-invariant functions $K\left[X_{\tau, Y}\right]^{T_{\tau}}$ in $K\left[X_{\tau, Y}\right]$ equals $K\left[\tau^{\perp}\right] \otimes_{K\left[\sigma^{\vee} \cap \tau^{\perp}\right]} K\left[\left[\sigma^{\vee} \cap \tau^{\perp}\right]\right]$. The ideal $I=I_{O_{\tau, Y}} \subset K\left[X_{\tau, Y}\right]$ of the orbit $O_{\tau, Y}$ is generated by all monomials with positive weights in the set $\sigma^{\vee}+\tau^{\vee} \subset \tau^{\vee}=\tau^{\vee} \oplus \tau^{\perp}$ and consequently it is generated by $\underline{\tau}^{\vee} \backslash\{0\}$. Thus $K\left[X_{\tau, Y}\right]^{T_{\tau}} \simeq K\left[O_{\tau, Y}\right]$ and $\lim _{\leftarrow} K\left[X_{\tau, Y}\right] / I^{k} \simeq K\left[O_{\tau, Y}\right]\left[\left[\underline{\tau}^{\vee}\right]\right]$. This gives $K\left[\widehat{Y}_{O_{\tau, Y}}\right] \simeq K\left(O_{\tau, Y}\right)\left[\left[\underline{\tau}^{\vee}\right]\right]$.

(2) Let $O_{\tau, Y} \in Y$ be the minimal orbit scheme containing $p$. First we prove that $\Gamma_{O_{\tau}}=\Gamma_{p}$. We have the obvious inclusion $\Gamma_{O_{\tau}} \subset \Gamma_{p}$. Now if $g \in \Gamma_{p}$ then for any $f \in K\left[O_{\tau}\right],(g(f)-f) \in I_{p}$, where $I_{p} \subset K\left[O_{\tau}\right]$ describes $p$. Since $\Gamma_{p}=\Gamma_{p^{\prime}}$ for any $p^{\prime} \in \Gamma \cdot p$, it follows that $(g(f)-f) \in \bigcap_{h \in T} h \cdot I_{p}=\{0\}$.

The ideal $I_{p} \subset \mathcal{O}_{p, O_{\tau}}$ of $p$ is generated by local parameters $u_{1}, \ldots, u_{l}$. Then there are $\Gamma_{p}$-equivariant isomorphisms $\widehat{\mathcal{O}}_{p, O_{\tau}} \simeq K_{p}\left[\left[u_{1}, \ldots, u_{l}\right]\right]$ and $\widehat{\mathcal{O}}_{p, Y} \simeq K_{p}\left[\left[u_{1}, \ldots, u_{l}\right]\right]\left[\left[\tau^{\vee}\right]\right]=K_{p}\left[\left[\left(\tau \oplus^{\Gamma_{p}}\left\langle e_{1}, \ldots, e_{l}\right\rangle\right)^{\vee}\right]\right]$ where $\widehat{\mathcal{O}}_{p, O_{\tau}}=\widehat{\mathcal{O}}_{p, Y}^{T_{\tau}}$. Therefore $\sigma_{p}=\tau \oplus^{\Gamma_{p}}\left\langle e_{1}, \ldots, e_{l}\right\rangle$ and $\underline{\operatorname{sing}^{\Gamma_{p}}}\left(\sigma_{p}\right)=\underline{\operatorname{sing}^{\Gamma_{\tau}}(\tau)}$.

By Lemmas 4.7 .2 and by 4.7 .3 the singularity type of a nonclosed point $p, \operatorname{sing}(p):=\operatorname{sing}\left(\sigma_{p}\right)$ (resp. $\operatorname{sing}^{\Gamma_{p}}(p):=\left(\Gamma_{p}, \operatorname{sing}^{\Gamma_{p}}\left(\sigma_{p}\right)\right)$, is uniquely determined. Moreover singularity type determines a stratification $\operatorname{Sing}(Y)$ (resp. $\operatorname{Sing}^{\Gamma}(Y)$ ) on $Y$ such that all points in the same stratum have the same singularity type. This yields

Lemma 4.7.4. Let $\Gamma$ act on $\widehat{X}_{\Delta}:=X_{\Delta} \times_{X_{\sigma}} \widehat{X}_{\sigma}$ There is a stratification $\operatorname{Sing}^{\Gamma}\left(\widehat{X}_{\Delta}\right)$ of $\widehat{X}_{\Delta}$ which is determined by singularity type and therefore preserved by any $\Gamma$-equivariant automorphism of $\widehat{X}_{\Delta}$.

4.8. Semicomplex associated to a stratified toroidal variety. For a $\Gamma$-semicone $\sigma$ we denote by $X_{\sigma}$ the associated stratified toric variety.

Definition 4.8.1. Let $(X, S)$ be a $\Gamma$-stratified toroidal variety. We say that a semicomplex $\Sigma$ is associated to $(X, S)$ if there is a bijection $\Sigma \rightarrow S$ with the following properties: Let $\sigma \in \Sigma$ map to $s=\operatorname{strat}_{X}(\sigma) \in S$. Then any $x \in s$ admits an open $\Gamma$-invariant neighborhood $U_{\sigma} \subset X$ and a $\Gamma$-equivariant $\Gamma_{s}$-smooth morphism $\phi_{\sigma}: U_{\sigma} \rightarrow X_{\sigma}$ of stratified varieties such that $s \cap U$ equals $\phi_{\sigma}^{-1}\left(O_{\sigma}\right)$ and the intersections $s^{\prime} \cap U, s^{\prime} \in S$, are precisely the inverse images of the strata of $X_{\sigma}$ and the action of $\Gamma$ on $X_{\sigma}$ extends the action of $\Gamma_{s}$.

We call the smooth morphisms $U_{\sigma} \rightarrow X_{\sigma}$ from the above definition charts. A collection of charts satisfying the conditions from the above definition is called an atlas.

Remarks. 1. Different $(X, S)$ may have the same associated semicomplex $\Sigma$. Smooth varieties endowed with the trivial stratification have the associated semicomplex consisting of one zero cone.

2. The action of $\Gamma_{s}=\Gamma_{\sigma}$ is fixed for $\sigma$ while the action of $\Gamma$ on $X_{\sigma}$ depends upon charts.

Lemma 4.8.2. For any $\Gamma$-stratified toroidal variety $(X, S)$ there exists a unique (up to isomorphism) associated $\Gamma$-semicomplex $\Sigma$. Moreover $\tau \leq \sigma$ iff $\overline{\operatorname{strat}_{X}(\tau)} \supset \operatorname{strat}_{X}(\sigma) .(X, S)$ is a toroidal embedding iff $\Sigma$ is a complex. 
Proof First we assign to any stratum $s$ a semicone $\sigma$.

By Definition 4.1.5 there is a $\Gamma_{s}$-smooth morphism $\phi_{\sigma}: U_{\sigma} \rightarrow X_{\sigma}$ into a stratifed toric variety $\left(X_{\sigma}, S_{\sigma}\right)$ preserving strata. Note that $\Gamma_{s}$ acts trivially on $O_{\sigma}$. So we can assume that $\sigma=\underline{\sigma}$ is of maximal dimension in $N_{\sigma}$ by composing $\phi$, if necessary, with a suitable projection.

We define the $\Gamma_{s}$-semicone $\sigma$ to be the $\Gamma_{s}$-semifan associated to the $\Gamma_{s}$-stratified toric variety $\left(X_{\sigma}, S_{\sigma}\right)$.

Then there is an open subset $U$ of $U_{\sigma}$ intersecting $s$ and a $\Gamma_{s}$-étale morphism $\phi:=\phi_{\sigma} \times \psi: U \rightarrow$ $X_{\sigma} \times \mathbf{A}^{k}=X_{\tau}$ preserving strata, where $\tau=\sigma \times\left\langle e_{1}, \ldots, e_{\operatorname{dim}(s)}\right\rangle$, and $\psi: U \rightarrow \mathbf{A}^{k}$ is a morphism defined by local parameters $u_{1}, \ldots, u_{k}$ on $s$. The stratification of $X_{\tau}$ is defined by the embedded semifan $\sigma \subset \bar{\tau}$, consisting of the faces of the semicone $\sigma$ in the lattice $N_{\tau}$. Then $\widehat{\phi}: \widehat{X}_{x} \rightarrow \widehat{X}_{\tau}$ is a $\Gamma_{s}$-equivariant isomorphism preserving strata. By Lemma 4.6.1 the embedded $\Gamma_{s}$-semifan $\sigma \subset \tau$ and the $\Gamma_{s}$-semicone $\sigma$ are determined uniquely up to isomorphism. We write $s=\operatorname{strat}_{X}(\sigma)$ and define $\Gamma_{\sigma}:=\Gamma_{s}$.

Assume that the closure of a stratum $s=\operatorname{strat}_{X}(\sigma)$ contains a stratum $t=\operatorname{strat}_{X}(\tau)$. Let $\phi_{\tau}: U_{\tau} \rightarrow X_{\tau}$ denote a chart associated with $\tau$. Then the stratum $s \cap U_{\tau}$ and the strata of $U_{\tau}$ having $s \cap U_{\tau}$ in their closure determine a semicone $\sigma_{s} \subset \bar{\tau}$. It follows from the uniqueness of $\sigma$ that there is a saturated embedding $i_{\sigma}^{\tau}$ of the semicone $\sigma$ into the semicone $\tau$ with the image $i_{\sigma}^{\tau}(\sigma)=\sigma_{s}$. Then we shall write $\sigma \leq \tau$.

The $\Gamma$-semicomplex $\Sigma$ is defined as the collection of the $\Gamma_{\sigma}$-semicones $\sigma$, and the saturated face inclusions $i_{\sigma}^{\tau}$ for $\sigma \leq \tau$.

Now let $\Sigma$ and $\Sigma^{\prime}$ be two semicomplexes associated to $(X, S)$. By uniqueness there are isomorphisms of semicones $j_{\sigma}: \sigma \rightarrow \sigma^{\prime}$ (see Definition 4.3.6). These isomorphisms induce an isomorphism of semicomplexes.

The second part follows from the fact that locally toroidal embeddings correspond to fans consisting of all faces of some cone.

Lemma 4.8.3. Let $\left(X_{\Sigma}, S\right)$ be a $\Gamma$-stratified toric variety corresponding to an embedded $\Gamma$-semifan $\Omega \subset \Sigma$. Then $\left(X_{\Sigma}, S\right)$ is a $\Gamma$-stratified toroidal variety with the associated $\Gamma$-semicomplex $\Omega^{\text {semic }}$.

There is an atlas

$$
\mathcal{U}^{\text {can }}\left(X_{\Sigma}, S\right)=\mathcal{U}(\Sigma, \Omega)
$$

on $\left(X_{\Sigma}, S\right)$ defined as follows: For any $\sigma$ in $N$ such that $\omega(\sigma)=\omega$ in $N_{\omega}$ there is a chart $\phi_{\sigma}: X_{\sigma} \rightarrow X_{\omega}$ given by any projection $\pi_{\sigma}^{\omega}: \sigma \rightarrow \omega$ such that $\pi_{\sigma \mid \omega}^{\omega}=\operatorname{id}_{\mid \omega}$.

Proof. Follows from Proposition 3.1.7 and from the definition of the associated semicomplex.

\subsection{Local properties of orientation.}

Definition 4.9.1. We shall call a proalgebraic group $G$ connected if it is a connected affine scheme. For any proalgebraic group $G=\lim _{\leftarrow} G_{i}$, denote by $G^{0}$ its maximal connected proalgebraic subgroup $G^{0}=\lim _{\leftarrow} G_{i}^{0}$.

Lemma 4.9.2. $G=\lim _{\leftarrow} G_{i}$ is connected if each $G_{i}$ is irreducible.

Proof. By Lemma 4.4 .2 we can assume all morphisms $G_{i} \rightarrow G_{j}$ to be epimorphisms and $K[G]=\bigcup K\left[G_{i}\right]$. If $g_{1}, g_{2} \in K[G]$ and $g_{1} \cdot g_{2}=0$ then $g_{1}, g_{2} \in K\left[G_{i}\right]$ for some $i$ and $g_{1}$ or $g_{2}$ equals zero.

Lemma 4.9.3. Let $G=\lim _{\leftarrow} G_{i}$ be a connected proalgebraic group. Then the image $\phi_{i}(G) \subset G_{i}$ of the natural homomorphism $\phi_{i}: G \rightarrow G_{i}$ is connected.

Definition 4.9.4. Let $X$ be a stratified (resp. $\Gamma$-stratified) toroidal scheme over $K$. We say that an automorphism (resp. $\Gamma_{x}$-equivariant automorphism) $\phi$ of $X$ preserves orientation at a $K$-rational point $x=\phi(x)$ if it induces an automorphism $\widehat{\phi} \in \operatorname{Aut}\left(\widehat{X}_{x}, S\right)^{0}\left(\operatorname{resp} . \widehat{\phi} \in \operatorname{Aut}^{\Gamma}\left(\widehat{X}_{x}, S\right)^{0}\right)$.

Definition 4.9.5. We say that two étale morphisms (resp. $\Gamma$-étale morphisms) of stratified toroidal schemes over $K, f_{1}, f_{2}:(X, S) \rightarrow(Y, T)$, determine the same orientation at a closed $K$-rational point $x \in X$ if $f_{1}(x)=f_{2}(x)$ and $\left(\widehat{f}_{2}\right)^{-1} \circ \widehat{f}_{1} \in \operatorname{Aut}\left(\widehat{X}_{x}, S\right)^{0}\left(\operatorname{resp} .\left(\widehat{f}_{2}\right)^{-1} \circ \widehat{f}_{1} \in \operatorname{Aut}^{\Gamma}\left(\widehat{X}_{x}, S\right)^{0}\right)$.

In further considerations we shall consider the case of $\Gamma$-stratified toroidal schemes. The case of stratified toroidal schemes corresponds to the situation when $\Gamma$ is a trivial group. 
Definition 4.9.6. Let $(X, S)$ is a $\Gamma$-stratified toroidal scheme and $x \in X$ be a $K$-rational point. We call functions $y_{1}, \ldots, y_{k}$ locally toric parameters if $y_{1}=\phi_{x}^{*}\left(u_{1}\right), \ldots, y_{k}=\phi_{x}^{*}\left(u_{k}\right)$, where $u_{1}, \ldots, u_{k}$ are semiinnvariant generators at the orbit point $O_{\sigma}$ on a $\Gamma$-stratified toric variety $X_{\sigma}$, and $\psi_{x}: U_{x} \rightarrow X_{\sigma}$ is a $\Gamma_{x}$-étale morphism from an open $\Gamma_{x}$-invariant neighborhood $U_{x}$ of $x$ such that $\psi_{x}(x)=O_{\sigma}$ and the intersections $s^{\prime} \cap U, s^{\prime} \in S$, are precisely the inverse images of strata of $X_{\sigma}$.

Lemma 4.9.7. Locally toric parameters exist for any $K$-rational point of a $\Gamma$-stratified toroidal scheme $X$.

Proof Let $s$ be the stratum through $x$. By Definition 4.1.6 there is a $\Gamma_{x}$-śmooth morphism from an open neighborhood $U_{x}$ of $x$ such that $s=\phi_{x}^{-1}\left(O_{\sigma}\right)$ and the intersections $s^{\prime} \cap U, s^{\prime} \in S$, are precisely the inverse images of strata of $X_{\sigma}$. Let $x_{1}, \ldots, x_{k}$ be local paramters of $s$ at $x$. Set $g: U \rightarrow \mathbf{A}^{1}$, where $g(x)=\left(x_{1}, \ldots, x_{k}\right)$. Then the morphism $\psi_{x}:=\phi_{x} \times g$ is $\Gamma_{x}$-étale and defines locally toric parameters at $x$.

Lemma 4.9.8. Let $(X, S)$ be a $\Gamma$-stratified toroidal scheme and $x \in X$ be a $K$-rational point. Let $y_{1}, \ldots, y_{n}$ be locally toric paramters at $x \in X$. The ideals of closures of the strata $I_{s} \subset \mathcal{O}_{x, X}$ are generated by subsets of $\left\{u_{1}, \ldots, u_{k}\right\}$.

Proof. It suffices to show the lemma for characters generating $\sigma^{\vee}$ on a $\Gamma$-stratified toric variety $X_{\sigma}$. Let $T$ be the big torus on $X_{\sigma}$. Each stratum $s$ is $T$-invariant irreducible hence contains a $\mathbf{T}$ - orbit $O_{\tau}$, where $\tau \preceq \sigma$. We conclude that $\bar{s}=\overline{O_{\tau}}$. But then $I_{\overline{O_{\tau}}} \subset K\left[X_{\sigma}\right]$ is generated by functions corresponding to those generating functionals of $\sigma^{\vee}$ which are not zero on $\tau$.

Lemma 4.9.9. 1. The group $\operatorname{Aut}^{\Gamma}\left(\widehat{X}_{x}, S\right)$ is connected for any smooth $\Gamma$-stratified toroidal scheme $(X, S)$ over $\operatorname{Spec}(K)$ and any $K$-rational point $x \in X^{\Gamma}$.

2. Any two $\Gamma$-étale morphisms $g_{1}:(X, S) \rightarrow(Y, R), g_{2}:(X, S) \rightarrow(Y, R)$ between smooth $\Gamma$-stratified toroidal schemes such that $g_{1}(x)=g_{2}(x)$ determine the same orientation at $x$.

Proof. (1) We can replace $X$ by $\mathbf{A}^{k}$ since $\left(\widehat{X}_{x}, S\right) \simeq\left(\widehat{\mathbf{A}^{k}}{ }_{0}, S_{A}\right)$ for a toric stratification $S_{A}$ on $\mathbf{A}^{k}$. Let $m$ be the maximal ideal of $0 \in \mathbf{A}^{k}$. The automorphism $g \in G:=\operatorname{Aut}^{\Gamma}\left(\widehat{\mathbf{A}}_{0}{ }_{0}, S_{A}\right)$ is defined by $\Gamma$-semiinvariant functions $g^{*}\left(x_{1}\right), \ldots, g^{*}\left(x_{k}\right)$, where $x_{1}, \ldots, x_{k}$ are the standard coordinates on $\mathbf{A}^{k}$ such that the $\Gamma$-weights of $g^{*}\left(x_{i}\right)$ and $x_{i}$, where $i=1, \ldots, k$, are equal. There is a birational map $\alpha: \mathbf{A}^{1} \rightarrow G$ defined by

$$
\alpha(z):=\left(x_{1}, \ldots, x_{k}\right) \mapsto\left((1-z) g^{*}\left(x_{1}\right)+z x_{1}, \ldots,(1-z) g^{*}\left(x_{k}\right)+z x_{k}\right) .
$$

Note that $\alpha(z)$ defines automorphisms for the open subset $U$ of $\mathbf{A}^{1}$, where the linear parts of coordinates of $\alpha(z)$ are linearly independent. By Lemma 4.9.8 the closure of each stratum $s$ is described by a subset of $\left\{x_{1}, \ldots, x_{k}\right\}$. Since $g$ preserves strata, $\bar{s}$ is described by the corresponding subset of $\left\{g^{*}\left(x_{1}\right), \ldots, g^{*}\left(x_{k}\right)\right\}$. Thus the corresponding coordinates of $\alpha(z), z \in U$, belong to the ideal $I_{\bar{s}}$. Since they are linearly independent of order 1 they generate the ideal $\frac{I_{\bar{s}}}{I_{\bar{s}} \cdot m}$ and by the Lemma of Nakayama they generate the ideal $I_{\bar{s}}$. Therefore the automorphisms $\alpha(z), z \in U$, preserve strata. The morphism $\alpha$ "connects" the identity $\alpha(1)=$ id to an arbitrary element $\alpha(0)=g \in \operatorname{Aut}^{\Gamma}\left(\widehat{X}_{x}, S\right)^{0}$.

(2) Follows from (1).

Lemma 4.9.10. Let $f:(X, S) \rightarrow(Y, R)$ be a $\Gamma$-smooth morphism of relative dimension $k$ between $\Gamma$ stratified toroidal schemes. Let $x \in X^{\Gamma}$ be a closed $K$-rational point. Let $\Gamma$ act trivially on $\mathbf{A}^{k}$ and $g_{1}, g_{2}$ : $X \rightarrow \mathbf{A}^{k}$ be any two $\Gamma$-equivariant morphisms such that $g_{1}(x)=g_{2}(x)=0$ and $f \times g_{i}:(X, S) \rightarrow(Y, R) \times \mathbf{A}^{k}$ are $\Gamma$-étale for $i=1,2$. Then $f \times g_{1}$ and $f \times g_{2}$ determine the same orientation at $x$.

Definition 4.9.11. We shall call such a morphism $f \times g$ an étale extension of $f$ and denote it by $\tilde{f}$.

Proof of 4.9.10. Let $y=f(x)$ and $y_{1}, \ldots, y_{l}$ be locally toric parameters at $y \in Y$. Let $x_{1}, \ldots, x_{k}$ be standard coordinates at $0 \in \mathbf{A}^{k}$. Set $v_{i}=f^{*}\left(y_{i}\right)$ for $i=1, \ldots, l$ and $w_{j}^{1}=g_{1}^{*}\left(x_{j}\right), w_{j}^{2}=g_{2}^{*}\left(x_{j}\right)$ for $j=1, \ldots, k$. Then $v_{1}, \ldots, v_{l}, w_{1}^{1}, \ldots, w_{k}^{1}$ and $v_{1}, \ldots, v_{l}, w_{1}^{2}, \ldots, w_{k}^{2}$ are locally toric parameters at $x$. The automorphism $\left(\widehat{f \times g_{1}}\right)_{x} \circ\left(\widehat{f \times g_{2}}\right)_{x}^{-1}: \widehat{X}_{x} \rightarrow \widehat{X}_{x}$ maps $w_{i}^{1}$ to $w_{i}^{2}$. We can find a linear automorphism $\alpha_{1} \in\left\{\operatorname{id}_{\mathrm{v}}\right\} \times \mathrm{Gl}(k)$, which preserves $v$-coordinates and acts nontrivially on $w_{i}^{2}$-coordinates so that $\alpha_{1}^{*}\left(v_{i}\right)=v_{i}$ and $\alpha_{1}^{*}\left(w_{i}^{2}\right)=w_{i}^{1}+z_{i}$, where $z_{i}$ are some functions from the ideal $\left(w_{1}^{2}, \ldots, w_{k}^{2}\right)^{2}+\left(v_{1}, \ldots, v_{l}\right)$. Clearly $\alpha_{1} \in \operatorname{Aut}^{\Gamma}\left(\widehat{X}_{x}, S\right)^{0}$. Now consider the morphism $\mathbf{A}^{1} \rightarrow \operatorname{Aut}^{\Gamma}\left(\widehat{X}_{x}, S\right)^{0}$ such that $\mathbf{A}^{1} \ni t \mapsto \phi_{t}$, where 
$\phi_{t}^{*}\left(v_{i}\right)=v_{i}$ and $\phi_{t}^{*}\left(w_{j}^{1}\right)=w_{j}^{1}+t \cdot z_{i}$. This shows that $\phi_{1} \in \operatorname{Aut}^{\Gamma}\left(\widehat{X}_{x}, S\right)^{0}$. Finally $\left(\widehat{f \times g}_{1_{x}}\right) \circ\left(\widehat{f \times g}_{2_{x}}\right)^{-1}=$ $\phi_{1}^{-1} \circ \alpha_{1} \in \operatorname{Aut}^{\Gamma}\left(\widehat{X}_{x}, S\right)^{0}$.

Definition 4.9.12. We say that two $\Gamma$-smooth morphisms $f_{1}, f_{2}:(X, S) \rightarrow(Y, R)$ of dimension $k$ determine the same orientation at a closed $K$-rational point $x \in X^{\Gamma}$ if $f_{1}(x)=f_{2}(x)$ and there exist étale extensions $e f_{1}, \tilde{f}_{2}:(X, S) \rightarrow(Y, R) \times \mathbf{A}^{k}$ which determine the same orientation at $x$.

Lemma 4.9.13. Let $f:(X, S) \rightarrow(Y, R)$ be a $\Gamma$-smooth morphism of $\Gamma$-stratified toroidal schemes over $K$. Let $x \in X^{\Gamma}$ and $y=f(x) \in Y^{\Gamma}$ be $K$-rational points and $s=f^{-1}(y) \in S$ be the stratum through $x$. Let $y_{1}, \ldots, y_{l}$ be locally toric $\Gamma_{x}$-semiinvariant parameters at $y$ and $v_{1}=f^{*}\left(y_{1}\right), \ldots, v_{l}=f^{*}\left(y_{l}\right), w_{1}, \ldots, w_{k}$ be locally toric $\Gamma_{x}$-semiinvariant parameters at $x$. Set $R:=K\left[\left[w_{1}, \ldots, w_{k}\right]\right]$. Then $\widehat{\mathcal{O}}_{X, x}=R\left[\left[v_{1}, \ldots, v_{l}\right]\right] / I$, where $I$ is the ideal span by binomial relations in $v_{i}$. $\operatorname{Aut}_{R} \Gamma_{(}\left(\widehat{X}_{x}, S\right)$ is a proalgebraic group of $\Gamma_{x}$-equivariant automorphisms preserving strata and the subring $R$, and the monomorphism $\beta: \operatorname{Aut}_{R}^{\Gamma}\left(\widehat{X}_{x}, S\right) \rightarrow \operatorname{Aut}^{\Gamma}\left(\widehat{X}_{x}, S\right)$ induces an isomorphism

$$
\bar{\beta}: \operatorname{Aut}_{R}^{\Gamma}\left(\widehat{X}_{x}, S\right) / \operatorname{Aut}_{R}^{\Gamma}\left(\widehat{X}_{x}, S\right)^{0} \rightarrow \operatorname{Aut}^{\Gamma}\left(\widehat{X}_{x}, S\right) / \operatorname{Aut}^{\Gamma}\left(\widehat{X}_{x}, S\right)^{0} .
$$

Proof. It suffices to construct a surjective morphism $\alpha: \operatorname{Aut}^{\Gamma}\left(\widehat{X}_{x}, S\right) \rightarrow \operatorname{Aut}_{R}^{\Gamma}\left(\widehat{X}_{x}, S\right)$ with connected fibers such that $\alpha \circ \beta=\operatorname{id}_{\operatorname{Aut}_{R}^{\Gamma}\left(\widehat{X}_{x}, S\right)}$. Any automorphism $\phi$ from $\operatorname{Aut}^{\Gamma}\left(\widehat{X}_{x}, S\right)$ maps $w_{1}, \ldots, w_{k}, v_{1}, \ldots, v_{l}$ to $w_{1}^{\prime}, \ldots, w_{k}^{\prime}, v_{1}^{\prime}, \ldots, v_{l}^{\prime}$. We put $\alpha(\phi)^{*}\left(w_{i}\right)=w_{i}, \alpha(\phi)^{*}\left(v_{j}\right)=v_{j}^{\prime}$. Then $\alpha(\phi)$ is an endomorphism since $w_{i}$ are algebraically independent of $v_{j}$. It is an automorphism since we can easily define an inverse homomorphism. The fiber $\alpha^{-1}(\alpha(\phi))$ consists of all elements of the type $\phi=\alpha(\phi) \circ \phi_{1}$, where $\phi_{1}^{*}\left(w_{i}\right)=w_{i}^{\prime}$ and $\phi_{1}^{*}\left(v_{j}^{\prime}\right)=v_{j}^{\prime}$. It is connected: for any two elements $\phi=\alpha(\phi) \circ \phi_{1}$ and $\phi^{\prime}=\alpha(\phi) \circ \phi_{1}^{\prime}$ we can define a rational map $\Phi: \mathbf{A}^{1} \rightarrow \operatorname{Aut}^{\Gamma}\left(\widehat{X}_{x}, S\right)$ by $\Phi(t)^{*}\left(v_{j}^{\prime}\right)=v_{j}^{\prime}, \Phi(t)^{*}\left(w_{i}\right)=t w_{i}+(1-t) w_{i}^{\prime}$. The latter is defined on an open subset $U \subset \mathbf{A}^{1}$ containing 0 and 1 .

Lemma 4.9.14. Let $\phi_{i}:(X, S) \rightarrow(Y, R)$ for $i=1,2$ be two $\Gamma$-smooth morphisms of $\Gamma$-stratified toroidal schemes such that $\phi_{1}(x)=\phi_{2}(x)=y \in Y$ for a $K$-rational point $x \in X^{\Gamma}$ and strata in $S$ are preimages of strata in $T$ and $\phi_{1}^{-1}(y)=\phi_{2}^{-1}(y)$. Assume there exists a smooth scheme $V$ with a trival action of $\Gamma$ and a $\Gamma$-equivariant morphism $g: X \rightarrow V$ such that $\phi_{i} \times g:(X, S) \rightarrow(Y, R) \times V$ are $\Gamma$-smooth. Define $X^{\prime}:=g^{-1}(g(x)), S^{\prime}:=\left\{s \cap X^{\prime} \mid s \in S\right\}$.

Then $\phi_{1}$ and $\phi_{2}$ determine the same orientation iff their restrictions $\phi_{i}^{\prime}:\left(X^{\prime}, S^{\prime}\right) \rightarrow(Y, R) d o$.

Proof. We can assume that $g$ is $\Gamma$-étale by replacing, if necessary, $(Y, R)$ with $(Y, R) \times \mathbf{A}^{m}$, where $m=$ $\operatorname{dim}(X)-\operatorname{dim}(Y)-\operatorname{dim}(V)$, and $\phi_{i}:(X, S) \rightarrow(Y, R)$ and $\phi_{i} \times g:(X, S) \rightarrow(Y, R) \times V$ with $\Gamma$-smooth morphisms $\widetilde{\phi}_{i}:(X, S) \rightarrow(Y, R) \times \mathbf{A}^{m}$ and $\Gamma$-étale morphisms $\widetilde{\phi}_{i} \times g:(X, S) \rightarrow(Y, R) \times \mathbf{A}^{m} \times V$.

Let $y=f(x)$ and $y_{1}, \ldots, y_{l}$ be locally toric $\Gamma$-semiinvariant parameters at $y \in Y$. Let $x_{1}, \ldots, x_{k}$ be locally toric parameters at $p:=g(x) \in V$. Set $v_{i}^{1}=\phi_{1}^{*}\left(y_{i}\right), v_{i}^{2}=\phi_{2}^{*}\left(y_{i}\right)$ for $i=1, \ldots, l$ and $w_{j}=g^{*}\left(x_{j}\right)$ for $j=1, \ldots, k$. Then $v_{1}^{1}, \ldots, v_{l}^{1}, w_{1}, \ldots, w_{k}$ and $v_{1}^{2}, \ldots, v_{l}^{2}, w_{1}, \ldots, w_{k}$ are locally toric parameters at $x$. The automorphism $\alpha:=\left(\widehat{\phi_{1} \times g}\right)_{x}^{-1} \circ\left(\widehat{\phi_{2} \times g}\right)_{x}: \widehat{X}_{x} \rightarrow \widehat{X}_{x}$ maps the first set of parameters onto the second one. Thus $\alpha$ belongs to the group of automorphisms $\operatorname{Aut}_{R}^{\Gamma}\left(\widehat{X}_{x}, S\right)$ preserving $R=K\left[\left[w_{1}, \ldots, w_{k}\right]\right]$. The restriction of each automorphism from $\operatorname{Aut}_{R}^{\Gamma}\left(\widehat{X}_{x}, S\right)$ to $X^{\prime}=\operatorname{Spec}\left(\widehat{\mathcal{O}}_{X, x} /\left(w_{1}, \ldots, w_{k}\right)\right)$ is an automorphism. On the other hand we can write $\widehat{\mathcal{O}}_{X, x}=\widehat{\mathcal{O}}_{X^{\prime}, x}\left[\left[w_{1}, \ldots, w_{k}\right]\right]$. Hence each automorphism in $\operatorname{Aut}_{K}^{\Gamma}\left(\widehat{X}_{x}^{\prime}, S^{\prime}\right)$ determines an automorphism in $\operatorname{Aut}_{R}^{\Gamma}\left(\widehat{X}_{x}, S\right)$. We come to a natural epimorphism of proalgebraic groups:

$$
\text { res : } \operatorname{Aut}_{R}^{\Gamma}\left(\widehat{X}_{x}, S\right) \rightarrow \operatorname{Aut}_{K}^{\Gamma}\left(\widehat{X}_{x}^{\prime}, S^{\prime}\right) \text {. }
$$

The kernel of res is a proalgebraic group $H$ consisting of all automorphisms $\beta \in \operatorname{Aut}_{R}^{\Gamma}\left(\widehat{X}_{x}, S\right)$ which can be written in the form $\beta\left(v_{i}^{1}\right)=v_{i}^{1}+r_{i}, \beta\left(w_{j}\right)=w_{j}$, where $r_{i} \in\left(w_{1}, \ldots, w_{k}\right) \cdot \widehat{\mathcal{O}}_{X, x}$. For any fixed $\beta$ and $t \in \mathbf{A}^{1}$ yield $r_{i}^{t}:=r_{i}\left(v_{1}^{1}, \ldots, v_{l}^{1}, t \cdot w_{1}, \ldots, t \cdot w_{k}\right)$ and $\beta^{t}\left(v_{i}^{1}\right)=v_{i}^{1}+r_{i}^{t}, \beta^{t}\left(w_{j}\right)=w_{j}$. This gives a morphism $t: \mathbf{A}^{1} \rightarrow H$ such that

$t(1)=\beta^{1}=\beta$ and $t(0)=\mathrm{id}$. Consequently, $H$ is connected and

$$
\operatorname{res}^{-1}\left(\operatorname{Aut}_{K}^{\Gamma}\left(\widehat{X}_{x}^{\prime}, S^{\prime}\right)^{0}=H \cdot\left(\operatorname{Aut}_{K}^{\Gamma}\left(\widehat{X}_{x}^{\prime}, S^{\prime}\right)^{0}=\operatorname{Aut}_{R}^{\Gamma}\left(\widehat{X}_{x}, S\right)^{0} .\right.\right.
$$


By the above and Lemma 4.9.13 the homomorphisms

$$
\operatorname{Aut}_{K}^{\Gamma}\left(\widehat{X}_{x}, S\right) \stackrel{\beta}{\longleftarrow} \operatorname{Aut}_{R}^{\Gamma}\left(\widehat{X}_{x}, S\right) \stackrel{\text { res }}{\longrightarrow} \operatorname{Aut}_{K}^{\Gamma}\left(\widehat{X}_{x}^{\prime}, S^{\prime}\right)
$$

define isomorphisms

$$
\operatorname{Aut}_{K}^{\Gamma}\left(\widehat{X}_{x}, S\right) / \operatorname{Aut}_{K}^{\Gamma}\left(\widehat{X}_{x}, S\right)^{0} \stackrel{\bar{\beta}^{-1}}{\longrightarrow} \operatorname{Aut}_{R}^{\Gamma}\left(\widehat{X}_{x}, S\right) / \operatorname{Aut}_{R}^{\Gamma}\left(\widehat{X}_{x}, S\right)^{0} \stackrel{\overline{\operatorname{res}}_{1}}{\longrightarrow} \operatorname{Aut}_{K}^{\Gamma}\left(\widehat{X}_{x}^{\prime}, S^{\prime}\right) / \operatorname{Aut}_{K}^{\Gamma}\left(\widehat{X}_{x}^{\prime}, S^{\prime}\right)^{0}
$$

Finally we see that $\left(\widehat{\phi_{1} \times g}\right)_{x} \circ\left(\widehat{\phi_{2} \times g}\right)_{x}^{-1} \in \operatorname{Aut}_{K}^{\Gamma}\left(\widehat{X}_{x}, S\right)^{0}$ iff ${\widehat{\phi_{1}^{\prime}}}_{x} \circ{\widehat{\phi_{2 x}^{\prime}}}^{-1} \in \operatorname{Aut}_{K}^{\Gamma}\left(\widehat{X}_{x}^{\prime}, S^{\prime}\right)^{0}$

\subsection{Orientation on stratified toroidal varieties.}

Definition 4.10.1. Let $(X, S)$ be a $\Gamma$-stratified toroidal variety (or $\Gamma$-stratified toroidal scheme) with an associated $\Gamma$-semicomplex $\Sigma$. Let $\tau \leq \sigma$ and $x \in s$. Let $\imath_{\tau}^{\sigma}: \tau \rightarrow \sigma$ denote the standard embedding and $\phi_{\sigma}: U_{\sigma} \rightarrow X_{\sigma}$ be a chart. For any $\sigma^{\prime} \preceq \sigma$ in $N_{\sigma}$ such that $\omega\left(\sigma^{\prime}\right)=\tau$ in $N_{\tau}$ we denote by $\phi_{\sigma}^{\sigma^{\prime}}: U_{\sigma}^{\sigma^{\prime}}:=$ $\phi_{\sigma}^{-1}\left(X_{\sigma^{\prime}}\right) \rightarrow X_{\sigma^{\prime}}$ the restriction of $\phi_{\sigma}$ to $U_{\sigma}^{\sigma^{\prime}}$.

Let $\pi_{\sigma^{\prime}}^{\tau}: X_{\sigma^{\prime}} \rightarrow X_{\tau}$ denote the toric morphism induced by any projection $\bar{\pi}_{\sigma^{\prime}}^{\tau}: \sigma^{\prime} \rightarrow \tau$ such that $\bar{\pi}_{\sigma^{\prime}}^{\tau} \circ \imath_{\tau}^{\sigma}=\mathrm{id}_{\mid \tau}$.

1. We say that the $\Gamma$-stratified toroidal variety $(X, S)$ with atlas $\mathcal{U}$ is oriented if for any two charts $\phi_{i}: U_{i} \rightarrow X_{\sigma_{i}}$, where $i=1,2$, and any $\sigma_{i}^{\prime} \preceq \sigma_{i}$ such that $\omega\left(\sigma_{i}^{\prime}\right)=\sigma \leq \sigma_{i}$ the $\Gamma_{\sigma^{-}}$smooth morphisms $\pi_{\sigma_{i}^{\prime}}^{\sigma} \phi_{\sigma_{i}}^{\sigma_{i}^{\prime}}: U_{\sigma_{i}}^{\sigma_{i}^{\prime}} \rightarrow X_{\sigma}$ determine the same orientation at any $x \in U_{\sigma_{1}}^{\sigma_{1}^{\prime}} \cap U_{\sigma_{2}}^{\sigma_{2}^{\prime}} \cap \operatorname{strat}_{X}(\sigma)$.

2. Let $(X, S)$ be a $\Gamma$-stratified toroidal scheme with atlas $\mathcal{U}$ which contains a reduced subscheme $W$ of finite type over $K$. We say that $(X, S)$ is oriented along $W$ if for any two charts $\phi_{i}: U_{i} \rightarrow X_{\sigma_{i}}$, where $i=1,2$, and any $\sigma_{i}^{\prime} \preceq \sigma_{i}$ such that $\omega\left(\sigma_{i}^{\prime}\right)=\sigma \leq \sigma_{i}$ the $\Gamma_{\sigma^{-}}$smooth morphisms $\pi_{\sigma_{i}^{\prime}} \phi_{\sigma_{i}}^{\sigma_{i}^{\prime}}: U_{\sigma_{i}}^{\sigma_{i}^{\prime}} \rightarrow X_{\sigma}$ determine the same orientation at any $x \in U_{\sigma_{1}}^{\sigma_{1}^{\prime}} \cap U_{\sigma_{2}}^{\sigma_{2}^{\prime}} \cap \operatorname{strat}_{X}(\sigma) \cap W$.

3. We shall call such $W$ a $K$-subscheme.

The following lemma is a generalization of Lemma 4.8.3

Lemma 4.10.2. Let $\left(X_{\Sigma}, S\right)$ be a $\Gamma$-stratified toric variety corresponding to an embedded semifan $\Omega \subset \Sigma$. Then $\left(X_{\Sigma}, S\right)$ is an oriented $\Gamma$-stratified toroidal variety with the associated oriented $\Gamma$-semicomplex $\Omega^{\text {semic }}$ and atlas $\mathcal{U}^{\text {can }}(\Omega, \Sigma)$.

Remark. By Lemma 4.9.10 the above definition does not depend upon the choice of the projection $\pi_{\sigma}$.

Definition 4.10.3. Let $(X, S)$ be a $\Gamma$-stratified toroidal variety (respectively a $\Gamma$-stratified toroidal scheme with a $K$-subscheme $W$ ) with two atlases $\mathcal{U}_{1}$ and $\mathcal{U}_{2}$ such that $\left(X, S, \mathcal{U}_{1}\right)$ and $\left(X, S, \mathcal{U}_{2}\right)$ are oriented (resp. oriented along $\mathrm{W})$. Then $\mathcal{U}_{1}$ and $\mathcal{U}_{2}$ on $(X, S)$ are compatible (resp. compatible along $\left.W\right)$ if $(X, S, \mathcal{U})$, where $\mathcal{U}:=\mathcal{U}_{1} \cup \mathcal{U}_{2}$, is oriented (resp. oriented along $W$ ).

Lemma 4.10.4. Let $f:(X, S) \rightarrow(Y, R)$ be a $\Gamma$-smooth morphism of $\Gamma$-stratified toroidal schemes such that the strata in $S$ are preimages of strata in $R$ and all strata in $R$ are dominated by strata in $S$. Assume that $(Y, R, \mathcal{U})$ with associated $\Gamma$-semicomplex $\Sigma$ is oriented along a $K$-subscheme $W$. Define $f^{*}(\mathcal{U}):=$ $\{\phi f \mid \phi \in \mathcal{U}\}$. Then $\left(X, S, f^{*}(\mathcal{U})\right)$ with the associated $\Gamma$-semicomplex $\Sigma$ is oriented along any $K$-subscheme $W^{\prime} \subset f^{-1}(W)$.

Proof. Let $\phi: U \rightarrow X_{\sigma}$ be a chart on $Y$. Then $\phi$ is $\Gamma$-equivariant, $\Gamma_{\sigma}$-smooth morphism such that the intersections $r \cap U$ are inverse images of strata of $X_{\sigma}$. Then $\phi f: \phi^{-1}(U) \rightarrow X_{\sigma}$ has exactly the same properties. Since $X_{\sigma} / / \Gamma_{\sigma}$ exists it follows from Lemma 4.1.3 that $\phi f$ is $\Gamma_{\sigma}$-smooth.

Lemma 4.10.5. Let $\phi_{i}:(X, S) \rightarrow(Y, R)$ for $i=1,2$ be two $\Gamma$-smooth morphisms of $\Gamma$-stratified toroidal schemes such that the strata in $S$ are preimages of strata in $R$. Assume $(Y, R, \mathcal{U})$ is oriented and there exists a $\Gamma$-equivariant morphism $g: X \rightarrow V$ into a smooth scheme with trivial action of $\Gamma$, such that $\phi_{i} \times g:(X, S) \rightarrow(Y, R) \times V$ are $\Gamma$-smooth. Set $X^{\prime}:=g^{-1}(g(x)), S^{\prime}:=S \cap X^{\prime}$. Let $W \subset X^{\prime}$ be a $K$-subscheme. Let $\psi_{i}:\left(X^{\prime}, S^{\prime}\right) \rightarrow(Y, R)$ denote the restrictions of $\psi_{i}$.

Then $\phi_{1}^{*}(\mathcal{U})$ and $\phi_{2}^{*}(\mathcal{U})$ are compatible on $(X, S)$ along $W$ iff $\psi_{1}^{*}(\mathcal{U})$ and $\psi_{2}^{*}(\mathcal{U})$ are compatible on $\left(X^{\prime}, S^{\prime}\right)$ along $W$.

Proof. Follows from Lemma 4.9.14. 
Example 4.10.6. The group of automorphisms of the formal completion of a toroidal embedding at a closed point is connected.

The formal completion of a toroidal embedding at a closed point is isomorphic to $\widehat{X}_{\sigma \times \operatorname{reg}(\sigma)}$, where $\operatorname{reg}(\sigma)$ is a regular cone and strata are defined by all faces of $\sigma$. Let $x_{1}, \ldots, x_{k}$ be semiinvariant coordinates on $\widehat{X}_{\sigma}$ corresponding to characters $m_{1}, \ldots, m_{k}$. Denote by $x_{k+1}, \ldots, z_{k+l}$ the standard coordinates on $X_{\operatorname{reg}(\sigma)}$ corresponfing to characters $m_{k+1}, \ldots, m_{k+l}$. Let $\phi$ be an automorphism of $\widehat{X}_{\sigma \times \operatorname{reg}(\sigma)}$ which preserves the torus orbit stratification. Since all the divisors in the orbit stratification are preserved, the divisors of $x_{i}$, where $i=1, \ldots, k$, are also preserved, so we have $\phi^{*}\left(x_{i}\right)=x_{i} s_{i}$, where $s_{i}=a_{i 0}+a_{i \alpha} x^{\alpha}+\ldots$ is invertible and $\alpha=\left(\alpha_{1}, \ldots, \alpha_{k+l}\right)$ denote multiindices. By Lemma 4.9.10, we may assume that $\phi^{*}\left(x_{i}\right)=x_{i}$ for $i=k+1, \ldots, l$. An integral vector $v:=\left(v_{1}, \ldots, v_{l+k}\right) \in \operatorname{int}(\sigma \times \operatorname{reg}(\sigma))$ defines the 1-parameter subgroup $t \mapsto t^{v}$ of the "big" torus $T$. For any $t \in T$ let $t^{v}$ denote the automorphism defined as

$$
x_{i} \mapsto x_{i} \cdot t^{\left\langle v, m_{i}\right\rangle} .
$$

consider the morphism $\phi_{t}:=t^{-v} \phi t^{v}$. In particular $\phi_{1}=\phi$ and

$$
x_{i} \circ \phi_{t}=x_{i}\left(a_{i 0}+t^{\alpha_{1} m_{1} v_{1}+\ldots+\alpha_{l+k} m_{l+k} v_{l+k}} a_{i \alpha} x^{\alpha}+\ldots\right) \text {, }
$$

where $\alpha_{1} m_{1} v_{1}+\ldots+\alpha_{l+k} m_{l+k} v_{l+k}=\left\langle v, \alpha_{1} m_{1}+\ldots+\alpha_{l+k} m_{l+k}\right\rangle \geq 0$. Then $\phi_{0}$ is the well defined automorphism $x_{i} \mapsto a_{i 0} x_{i}$. The latter automorphism belongs to the torus $T$. Since we can "connect" any automorphism $\phi$ with an element of the torus the connectedness of the group of automorphisms follows.

Any toroidal embedding is an oriented stratified variety.

By Lemma 4.9.14 and by the connectedness of the automorphism group any two charts determine the same orientation.

Example 4.10.7. Let $X:=\left\{x \in \mathbf{A}^{4} \mid x_{1} x_{2}=x_{3} x_{4}\right\}$ be a toric variety with isolated singularity. Then $X$ is a stratified toroidal variety with the stratification consisting of the singular point $p$ and of its complement. The blow-up of the ideal of the point is a resolution of singularities with the exceptional divisor determining the valuation. Consequently the valuation determined by the point is preserved by all automorphisms in $\operatorname{Aut}\left(\widehat{X}_{x}, S\right)$. We have the natural homomorphism $d: \operatorname{Aut}\left(\widehat{X}_{x}, S\right) \rightarrow \operatorname{Gl}\left(\operatorname{Tan}_{X, x}\right)$. The kernel of $d$ is a connected proalgebraic group (see Lemma 7.3.1(3)). The image $d\left(\operatorname{Aut}\left(\widehat{X}_{x}, S\right)\right)$ consists of linear automorphisms preserving the ideal of $x_{1} x_{2}-x_{3} x_{4}$, that is, multiplying the polynomial by a nonzero constant. Hence $d\left(\operatorname{Aut}\left(\widehat{X}_{x}, S\right)\right)=K^{*} \cdot O$, where $K^{*}$ acts by multiplying the coordinates by $t \in K^{*}$ and $O$ is the group of linear automorphisms preserving $x_{1} x_{2}-x_{3} x_{4}$. By the linear change of coordinates $x_{1}=y_{1}-i y_{2}, x_{2}=y_{1}+i y_{2}$, $x_{3}=y_{3}-i y_{4}, x_{4}=y_{3}+i y_{4}$ we transform the polynomial into $y_{1}^{2}+y_{2}^{2}+y_{3}^{2}+y_{4}^{2}(\operatorname{char}(K) \neq 2)$. This shows that $O$ is conjugate to the group of orthogonal matrices with $K$-rational coefficients $O(4, K) . O(4, K)$ consists of two components with $O(4, K)^{0}=S O(4, K)$. Therefore Aut $\left(\widehat{X}_{x}, S\right)=k e r(d) \cdot K^{*} \cdot O$ consists of two components. Fix any isomorphism $\phi: X \rightarrow X_{\sigma}$, which can be considered as a chart. An oriented atlas consists of charts compatible with $\phi$. Consequently, there are two orientations corresponding to any two incompatible isomorphisms $\phi: X \rightarrow X_{\sigma}$.

\subsection{Subdivisions of oriented semicomplexes.}

Definition 4.11.1. For any $\Gamma$-semicone $\sigma$ denote by $\operatorname{Aut}(\sigma)^{0}$ the group of all its automorphisms which define the $\Gamma$-equivariant automorphisms of $X_{\sigma}$ preserving orientation.

By an oriented semicomplex (resp. oriented $\Gamma$-semicomplex) we mean a semicomplex (resp. $\Gamma$-semicomplex) $\Sigma$ for which for any $\sigma \leq \tau \leq \gamma$ there is $\alpha_{\sigma} \in \operatorname{Aut}(\sigma)^{0}$ for which $\imath_{\tau}^{\gamma} \imath_{\sigma}^{\tau}=i_{\sigma}^{\gamma} \alpha_{\sigma}$.

Definition 4.11.2. By an isomorphism of two oriented $\Gamma$-semicomplexes $\Sigma \rightarrow \Sigma^{\prime}$ we mean a bijection of faces $\Sigma \ni \sigma \mapsto \sigma^{\prime} \in \Sigma^{\prime}$ along with a collection of face isomorphisms $j_{\sigma}: \sigma \rightarrow \sigma^{\prime}$ such that for any $\tau \leq \sigma$, there is an automorphism $\beta_{\tau} \in \operatorname{Aut}(\tau)^{0}$ such that $j_{\sigma} \imath_{\tau}^{\sigma}=\imath_{\tau^{\prime}}^{\sigma^{\prime}} j_{\tau} \beta_{\tau}$.

Remarks. 1. An oriented semicomplex can be viewed as an oriented $\Gamma$-semicomplex with trivial groups $\Gamma_{\sigma}$.

2. We will show that the $\Gamma$-semicomplex associated to an oriented $\Gamma$-stratified toroidal variety is oriented (Lemma 8.2.5).

3. The description of the group Aut $(\sigma)^{0}$ is given in Lemma 8.1.2.

Lemma 4.11.3. Let $\Delta$ be a subdivision of a fan $\Sigma$ and $\sigma \in \Sigma$. Then $\Delta \mid \sigma:=\{\delta \in \Delta \mid \delta \subset \sigma\}$ is a subdivision of $\sigma$. 
Definition 4.11.4. A subdivision of an oriented $\Gamma$-semicomplex $\Sigma$ is a collection $\Delta$ of fans $\Delta^{\sigma}$ in $N_{\sigma}$ where $\sigma \in \Sigma$ such that

1. For any $\sigma \in \Sigma, \Delta^{\sigma}$ is a subdivision of $\bar{\sigma}$ which is $\operatorname{Aut}(\sigma)^{0}$-invariant.

2. For any $\tau \leq \sigma, \Delta^{\sigma} \mid \bar{\tau}=\imath_{\tau}^{\sigma}\left(\Delta^{\tau}\right)$.

Remarks. 1. By abuse of terminology we shall understand by a subdivision of a semicone $\sigma$ a subdivision of the fan $\bar{\sigma}$.

2. A subdivision of an oriented semicomplex can be viewed as a subdivision of an oriented $\Gamma$-semicomplex with trivial groups $\Gamma_{\sigma}$.

3. By definition vectors in faces $\sigma$ of an oriented $\Gamma$-semicomplex $\Sigma$ are defined up to automorphisms from $\operatorname{Aut}(\sigma)^{0}$. Consequently, the faces of subdivisions $\Delta^{\sigma}$ are defined up to automorphisms from $\operatorname{Aut}(\sigma)^{0}$ and in general don't give a structure of semicomplex. Only invariant faces of $\Delta^{\sigma}$ may define a semicomplex.

4. The condition on $\Delta^{\sigma}$ to be $\operatorname{Aut}(\sigma)^{0}$-invariant is for canonical subdivisions replaced with a somewhat stronger condition of similar nature which says that the induced morphism $\widehat{X}_{\Delta^{\sigma}}:=X_{\Delta^{\sigma}} \times_{X_{\sigma}} \widehat{X}_{\sigma} \rightarrow \widehat{X}_{\sigma}$ is $\operatorname{Aut}^{\Gamma}\left(\widehat{X}_{\sigma}\right)^{0}$-equivariant. The latter condition can be translated into the condition that all "new" rays of the subdivision are in the stable support of $\Sigma$.

Definition 4.11.5. Let $\Delta$ be a subdivision of an oriented $\Gamma$-semicomplex $\Sigma$. Let $\varrho_{\sigma}$ be an ray passing through the interior of the cone $\sigma \in \Sigma$ such that defining a collection of rays $\varrho:=\left\{\varrho_{\tau} \in \tau \mid \tau \geq \sigma\right\}$ such that for any $\tau \geq \sigma$, the ray $\varrho_{\tau}=\imath_{\sigma}^{\tau}\left(\varrho_{\sigma}\right)$ is Aut $(\tau)^{0}$-invariant . By the star subdivision of $\Delta$ at $\rho$ we mean the subdivision

$$
\varrho \cdot \Delta:=\left\{\varrho_{\tau} \cdot \Delta^{\tau} \mid \tau \geq \sigma\right\} \cup\left\{\Delta^{\tau} \mid \tau \nsupseteq \sigma\right\} .
$$

\subsection{Toroidal morphisms of stratified toroidal varieties.}

Definition 4.12.1. Let $(X, S)$ be a a stratified toroidal variety (resp. a $\Gamma$-stratified toroidal variety) with an atlas $\mathcal{U}$. We say that $Y$ is a toroidal modification of $(X, S)$ if

1. There is given a proper morphism $f: Y \rightarrow X$ (resp. proper $\Gamma$-equivariant morphism) such that for any $x \in s=\operatorname{strat}_{X}(\sigma)$ there exists a chart $x \in U_{\sigma} \rightarrow X_{\sigma}$, a subdivision $\Delta^{\sigma}$ of $\sigma$, and a fiber square

$$
\begin{array}{cccc}
U_{\sigma} & \stackrel{\phi_{\sigma}}{\longrightarrow} & X_{\sigma} \\
\uparrow f & & \uparrow \\
U_{\sigma} \times_{X_{\sigma}} X_{\Delta^{\sigma}} \simeq f^{-1}\left(U_{\sigma}\right) \stackrel{\phi_{\sigma}^{f}}{\longrightarrow} & X_{\Delta^{\sigma}}
\end{array}
$$

2. For any point $x$ in a stratum $s$ every automorphism (resp. $\Gamma_{s}$-equivariant automorphism) $\alpha$ of $\widehat{X}_{x}$ preserving strata and orientation can be lifted to an automorphism (resp. $\Gamma_{s}$-equivaiant automorphism) $\alpha^{\prime}$ of $Y \times_{X} \widehat{X}_{x}$.

Definition 4.12.2. Let $Y$ be a toroidal modification of a stratified toroidal variety $(X, S)$. By a canonical stratification $R$ on $Y$ we mean the finest stratification on $Y$ satisfying the conditions:

1. Strata of $R$ are mapped onto strata of $S$.

2. For any chart $\phi_{\sigma}: U_{\sigma} \rightarrow X_{\sigma}$ on $(X, S)$ there is an embedded subdivision $\Omega^{\sigma} \subset \Delta^{\sigma}$ such that the strata of $R \cap f^{-1}\left(U_{\sigma}\right)$ are defined as inverse images $\left(\phi_{\sigma}^{f}\right)^{-1}(\operatorname{strat}(\omega))$ of strata on $\left(X_{\Delta^{\sigma}}, S_{\Omega^{\sigma}}\right)$.

3. For any point $x$ in a stratum $s$ every $\Gamma_{s}$-equivariant automorphism $\alpha$ of $\widehat{X}_{x}$ preserving strata and orientation can be lifted to an $\Gamma_{s}$-equivariant automorphism $\alpha^{\prime}$ of $Y \times_{X} \widehat{X}_{x}$ preserving strata.

If $Y$ is a toroidal modification of $(X, S)$ and $R$ is a canonical stratification on $Y$ then we shall also speak of a toroidal morphism $(Y, R) \rightarrow(X, S)$ of stratified toroidal varieties.

Remarks. 1. The definition of a toroidal morphism of stratified toroidal varieties is a generalization of the definition of a toroidal morphism of toroidal embeddings. 
2. The condition (2) of Definition 4.12.1 is similar to Hironaka's "allowability" condition, used by Mumford in the theory of toroidal embeddings (see 39 and section 1.1). It means that a toroidal morphism, which by condition (1) is induced locally by charts, in fact does not depend on the charts i.e., if a morhism which satisfies the Hironaka condition is induced locally by the diagram (1) for some chart, then it is also induced locally by any other chart and the given subdivision (see Lemma 4.12.11).

3. In the above definition $\Omega^{\sigma}$ consists of those faces of $\Delta^{\sigma}$ whose relative interior intersect the stable support (see Lemma 6.5.1).

4. Only some special subdivisions $\Delta^{\sigma}$ of $\sigma$ define (locally) toroidal modifications (see example 4.12.3). These subdivisions, called canonical, induce modifications which are independent of the charts. They are characterized by the property that "new" rays of the subdivision are contained in the stable support (Proposition 7.6.1).

Example 4.12.3. Let $X=\mathbf{A}^{2}$ and $S$ be the stratification on $X$ that consists of the point $s_{0}=(0,0)$ and its complement $s_{1}=\mathbf{A}^{2} \backslash\{(0,0)\}$. Then $(X, S)$ is an oriented stratified toroidal variety corresponding to the semicomplex $\Sigma=\left\{\sigma_{0}, \sigma_{1}\right\}$ where $\sigma_{0}=\langle(1,0),(0,1)\rangle, \sigma_{1}=\{(0,0)\}$.

- Let $Y \rightarrow X$ be a toric morphism corresponding to the normalized blow-up of $I=\left(x^{2}, y\right)$. This morphism of toric varieties corresponds to the subdivision $\Delta:=\langle(1,2)\rangle \cdot \Sigma$ of $\Sigma$. Therefore condition (1) of Definition 4.12.1 is satisfied for the identity chart.

The condition (2) of the definition is not satisfied. The automorphism $\alpha$ defined by $\alpha(x)=y$, $\alpha(y)=x$ doesn't lift.

- Let $Y \rightarrow X$ be a blow-up of $I=(x, y)$. Then $Y$ is a toric variety and let $S_{Y}$ be the stratification consisting of $s_{1}$ and the toric orbits in the exceptional curve $\mathbf{P}^{1}: s_{2}=\{0\}, s_{3}=\{\infty\}$ and $s_{4}=$ $\mathbf{P}^{1} \backslash\{0\} \backslash\{\infty\}$. Then $Y$ is a toroidal modification of $(X, S)$. The automorphism $\alpha$ defined by $\alpha(x)=y$, $\alpha(y)=x$ lifts to the automorphism permuting $s_{2}$ and $s_{3}$. Therefore $\left(Y, S_{Y}\right) \rightarrow(X, S)$ is NOT a toroidal morphism.

- Let $Y \rightarrow X$ be a blow-up of $I=(x, y)$ and $S_{Y}$ be the stratification consisting of the exceptional curve $s_{2}=D$ and its complement $s_{1}$. Then $\left(Y, S_{Y}\right) \rightarrow(X, S)$ is a toroidal morphism corresponding to the subdivision $\Delta$ of $\Sigma$, where $\Delta=\left\{\langle(1,0),(1,1)\rangle,\langle(1,1)\rangle,\langle(1,1),(0,1)\rangle,\{(0,0)\}\right.$. The stratification $S_{Y}$ is described by the semicomplex $\Omega=\{\langle(1,1)\rangle,\{(0,0)\}\} \subset \Delta$. After blow-up some faces "disappear" from $\Omega \neq \Delta$. The remaining faces of $\Omega$ are $\operatorname{Aut}(\sigma)^{0}$-invariant.

Lemma 4.12.4. Let $Y \rightarrow X$ be a toroidal modification of an oriented $\Gamma$-stratified toroidal variety $(X, S)$. Then all fibers $f^{-1}(x)$, where $x \in s$, are isomorphic.

Proof. It follows from condition (1) of Definition 4.12.1 that all fibers $f^{-1}(x)$, where $x \in s=\operatorname{strat}_{X}(\sigma) \cap U_{\sigma}$, are isomorphic for any chart $U_{\sigma} \rightarrow X_{\sigma}$.

Lemma 4.12.5. Let $Y \rightarrow X$ be a toroidal modification of an oriented $\Gamma$-stratified toroidal variety $(X, S)$. Then the exceptional divisors of the morphism $f: Y \rightarrow X$ dominate strata.

Proof. Suppose an exceptional divisor $D$ does not dominate a stratum. The generic point of $f(D)$ is in a stratum $s$ and $\operatorname{dim}(f(D))<\operatorname{dim}(s)$. By Lemma 4.12.4, $s$ is not a generic open stratum in $X$. The dimension of a generic fiber $f^{-1}(x)$, where $x \in f(D)$, is greater than or equal to $\operatorname{dim}(D)-\operatorname{dim}(f(D))=n-1-\operatorname{dim}(f(D)$. The dimension of a generic fiber $f^{-1}(x)$, where $x \in s$, is at most $\operatorname{dim}\left(f^{-1}(s)\right)-\operatorname{dim}(s)<n-1-\operatorname{dim}(f(D)$. This contradicts Lemma 4.12.4.

Definition 4.12.6. If $\Sigma$ is a fan then we denote by $\operatorname{Vert}(\Sigma)$ the set of all 1-dimensional faces (rays) in $\Sigma$.

Lemma 4.12.7. Let $\sigma=\tau_{1} \oplus \tau_{2}$ and $\Delta$ be a subdivision of $\tau_{1}$. Then

$\Delta \oplus \tau_{2}:=\left\{\delta \oplus \sigma \mid \delta \in \Delta, \sigma \preceq \tau_{2}\right\}$ is a subdivision of $\tau$ called the joint of $\Delta$ and $\tau_{2}$.

Lemma 4.12.8. Let $\Omega \subset \Sigma$ be an embedded $\Gamma$-semifan. Let $\Delta$ be a subdivision of $\Sigma$ such that any ray $\varrho \in \operatorname{Vert}(\Delta \backslash \Sigma)$ passes through the interior of $\omega \in \Omega$. Then for any cone $\sigma=\omega(\sigma) \oplus \operatorname{r}(\sigma)$ in $\Sigma, \Delta \mid \sigma=$ $(\Delta \mid \omega(\sigma)) \oplus \mathrm{r}(\sigma)$.

Proof. Since $\omega:=\omega(\sigma)$ is a maximal face of $\sigma$ which belongs to $\Omega$, all "new" rays which are contained in $\sigma$ are in $\omega$ and therefore the maximal cones in $\Delta \mid \sigma$ are of the form $\tau=\omega \oplus \mathrm{r}(\sigma)$ and $\tau \subset \omega$. This shows that $\Delta_{1}:=\Delta \mid \sigma$ is a subfan of $\Delta_{2}:=(\Delta \mid \omega) \oplus \mathrm{r}(\sigma)$. By properness we get $\left|\Delta_{1}\right|=\left|\Delta_{2}\right|$ and hence $\Delta_{1}=\Delta_{2}$. 
Lemma 4.12.9. . Let $\psi_{1}: Y_{1} \rightarrow X$ and $\psi_{2}: Y_{2} \rightarrow X$ be proper birational morphisms of normal noetherian schemes.

1. Suppose $\widehat{X}_{x} \times_{X} Y_{1}$ and $\widehat{X}_{x} \times_{X} Y_{2}$ are isomorphic over $\widehat{X}_{x}$ for a $K$-rational point $x \in X$. Then there exists an open neighborhood $U$ of $x$ such that the proper birational map $\psi_{1}^{-1}(U) \rightarrow \psi_{2}^{-1}(U)$ is an isomorphism.

2. Suppose $\widehat{X}_{x} \times_{X} Y_{1} \rightarrow \widehat{X}_{x} \times_{X} Y_{2}$ is a proper morphism over $\widehat{X}_{x}$ for a $K$-rational point $x \in X$. Then there exists an open neighborhood $U$ of $x$ such that the proper birational map $\psi_{1}^{-1}(U) \rightarrow \psi_{2}^{-1}(U)$ is a morphism.

Proof (1) Suppose that the sets $\psi_{1}^{-1}(U)$ and $\psi_{2}^{-1}(U)$ are not isomorphic for any $U$. Then the closed subset of $Y_{2}$ where $\psi_{1} \psi_{2}^{-1}$ is not an isomorphism interesect the fiber $\psi_{2}^{-1}(x)$.

Let $Y_{3}$ be a component in $Y_{1} \times_{X} Y_{2}$ which dominates $X$. Since all varieties are normal, at least one of the proper morphisms $Y_{3} \rightarrow Y_{i}$ is not an isomorphism for $i=1,2$ over $\psi_{i}^{-1}(U)$ for any $U \ni x$. Then it collapses a curve to a point over $x$.

Let $\widehat{X}_{3}$ be a component in $\widehat{X}_{1} \times_{\widehat{X}_{x}} \widehat{X}_{2}$ which dominates $\widehat{X}_{x}$. The morphisms $\widehat{X}_{3} \rightarrow \widehat{X}_{i}$ for $i=1,2$ are pull-backs of the morphisms $Y_{3} \rightarrow Y_{i}$ via étale morphisms. This shows that they are not both isomorhisms, and neither is the induced birational map $\widehat{X}_{1} \rightarrow \widehat{X}_{2}$, which contradicts the assumption.

(2) The same reasoning.

Lemma 4.12.10. Let $\phi_{\sigma}: U_{\sigma} \rightarrow X_{\sigma}$ denote a chart on an oriented $\Gamma$-stratified toroidal variety $(X, S)$. Let $\sigma^{\prime} \preceq \sigma$ denote a face for which $\omega\left(\sigma^{\prime}\right)=\varrho \leq \sigma$.

Consider the smooth morphism $\psi_{\sigma}:=\pi_{\sigma^{\prime}}^{\varrho} \phi_{\sigma}^{\sigma^{\prime}}: U_{\sigma^{\prime}} \rightarrow X_{\varrho}$ induced by the chart $\phi_{\sigma}$.

Let $\Delta^{\sigma}$ be a subdivision of $\sigma$ and $\Delta^{\varrho}:=\Delta^{\sigma} \mid \varrho$ be a subdivision of $\varrho$.

Suppose $f_{\sigma}: \widetilde{U}_{\sigma}:=U_{\sigma} \times_{X_{\sigma}} X_{\Delta^{\sigma}} \rightarrow U_{\sigma}$ satisfies Condition (2) of Definition 4.12.1. Then the following diagram consists of two fiber squares of smooth morphisms:

$$
\begin{aligned}
& U_{\sigma}^{\sigma^{\prime}} \quad \longrightarrow \quad X_{\sigma^{\prime}}=X_{\varrho \oplus \mathrm{r}\left(\sigma^{\prime}\right)} \quad \longrightarrow \quad X_{\varrho} \\
& \uparrow f \quad \uparrow \quad \uparrow \\
& U_{\sigma}:=U \times_{X_{\sigma^{\prime}}} X_{\Delta^{\sigma}} \quad \simeq f^{-1}\left(U_{\sigma}^{\sigma^{\prime}}\right) \quad \rightarrow \quad X_{\Delta^{\sigma} \mid \sigma^{\prime}}=X_{\imath_{\varrho}^{\sigma}(\Delta \varrho) \oplus \mathrm{r}\left(\sigma^{\prime}\right)} \quad \rightarrow \quad X_{\Delta^{\varrho}}
\end{aligned}
$$

Proof It follows from Lemma 4.12.5 that the assumption of Lemma 4.12 .8 is satisfied for $\Delta^{\sigma}$. By Lemma 4.12.8, $\Delta^{\sigma}\left|\sigma^{\prime}=\Delta^{\sigma}\right| \varrho \oplus \mathrm{r}\left(\sigma^{\prime}\right)=\imath_{\varrho}^{\sigma}\left(\Delta^{\varrho}\right) \oplus \mathrm{r}\left(\sigma^{\prime}\right)$.

Lemma 4.12.11. Let $\phi_{\sigma}: U_{\sigma} \rightarrow X_{\sigma}$ and $\phi_{\tau}: U_{\tau} \rightarrow X_{\tau}$ denote two charts on an oriented $\Gamma$-stratified toroidal variety $(X, S)$. Let $\Delta^{\sigma}$ be a subdivision of $\sigma$ and $\Delta^{\tau}$ denote a subdivision of $\tau$. Assume that for any $\varrho \leq \sigma, \tau, \Delta^{\sigma}\left|\varrho=\Delta^{\tau}\right| \varrho$. Suppose $f_{\sigma}: \widetilde{U}_{\sigma}:=U_{\sigma} \times_{X_{\sigma}} X_{\Delta^{\sigma}} \rightarrow U_{\sigma}$ and $f_{\tau}: \widetilde{U}_{\tau}:=U_{\tau} \times_{X_{\tau}} X_{\Delta^{\tau}} \rightarrow U_{\tau}$ satisfy Condition (2) of Definition 4.12.1.

Then $\phi_{\sigma}^{-1}(U)$ and $\phi_{\tau}^{-1}(U)$ are isomorphic over $U:=U_{\sigma} \cap U_{\tau}$.

Proof. Suppose that the relevant sets are not isomorphic over $\operatorname{Spec}\left(\mathcal{O}_{x}\right)$ for $x \in \operatorname{strat}_{X}(\varrho) \cap U$. Then there are faces $\sigma^{\prime} \preceq \sigma$ and $\tau^{\prime} \preceq \tau$ such that $\omega\left(\sigma^{\prime}\right)=\omega\left(\tau^{\prime}\right)=\varrho$.

Set by $\operatorname{reg}(\varrho)=\left\langle e_{1}, \ldots, e_{\operatorname{dim}\left(\operatorname{strat}_{X}(\varrho)\right)}\right\rangle$. The diagram from Lemma 4.12 .10 gives the following fiber square diagram of étale extensions:

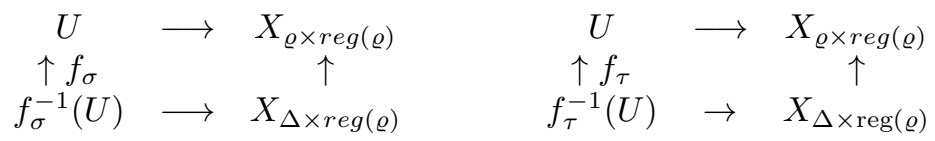

where the horizontal morphisms are étale.

Consider the following fiber square diagram:

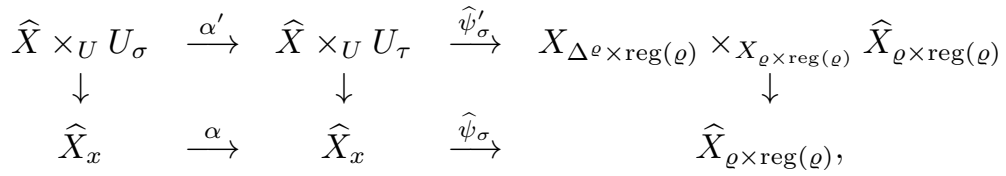


where $\widehat{\psi}_{\sigma}$ and $\widehat{\psi}_{\tau}$ are isomorphisms of the completions of the local schemes induced by étale extensions of the smooth morphisms $\psi_{\sigma}$ and $\psi_{\tau}, \alpha$ is an isomorphism such that $\widehat{\psi}_{\tau}:=\widehat{\psi}_{\sigma} \circ \alpha$, and $\widehat{\psi}_{\sigma}^{\prime}, \widehat{\psi}_{\tau}^{\prime}$ and $\alpha^{\prime}$ are liftings of $\widehat{\psi}_{\sigma}, \widehat{\psi}_{\tau}$ and $\alpha$.

It follows from the diagram that $\alpha$ is an automorphism of $\widehat{X}_{x}$ which can be lifted to an automorphism $\alpha^{\prime}$ of $\widehat{X}_{1}$. Note that $\alpha$ preserves strata and orientation. It follows that $\widehat{X} \times_{U} U_{\sigma}$ and $\widehat{X} \times_{U} U_{\tau}$. are isomorphic. By Lemma 4.12.9, $\widetilde{U}_{\sigma}$ and $\widetilde{U}_{\tau}$ are isomorphic over a neighborhoood of $x \in X$, which contradicts the choice of $x \in X$.

4.13. Canonical subdivisions of oriented semicomplexes. As a corollary from the proof of Lemma 4.12.11 we obtain the following lemma.

Lemma 4.13.1. Let $(X, S)$ be an oriented $\Gamma$-stratified toroidal variety of dimension $n$ with associated oriented $\Gamma$-semicomplex $\Sigma$ and let $f: Y \rightarrow(X, S)$ be a toroidal modification. Let $x$ be a closed point in stratum $\operatorname{strat}_{X}(\sigma) \in S, \phi_{\sigma}: U \rightarrow X_{\sigma}$ be a chart of a neighborhood $U$ of $x$ and $\Delta^{\sigma}$ be a subdivision of $\sigma$ for which there is a fiber square

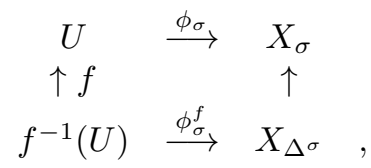

where the horizontal morphisms are $\Gamma_{\sigma}$-smooth. Set

$$
\operatorname{reg}(\sigma):=\left\langle e_{1}, \ldots, e_{n-\operatorname{dim}\left(N_{\sigma}\right)}\right\rangle=\left\langle e_{1}, \ldots, e_{\operatorname{dim}\left(\operatorname{strat}_{X}(\sigma)\right)}\right\rangle .
$$

Then

1. there is a fiber square of étale extensions

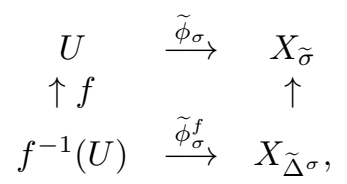

where the horizontal morphisms are $\Gamma_{\sigma}$-étale and where

$$
\tilde{\sigma}:=\bar{\sigma} \times \operatorname{reg}(\sigma) \text {, and } \widetilde{\Delta}^{\sigma}:=\Delta^{\sigma} \times \operatorname{reg}(\sigma) .
$$

2. $X_{\widetilde{\sigma}}$ is a $\Gamma_{\sigma}$-stratified toric variety with the strata described by embedded $\Gamma_{\sigma}$-semifan $\sigma \subset \widetilde{\sigma}$. Moreover the strata on $U$ are exactly inverse images of strata of $X_{\widetilde{\sigma}}$.

3. There is a fiber square of isomorphisms

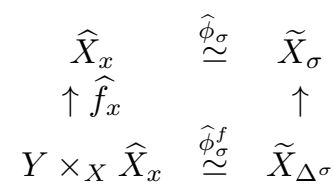

where

$$
\widetilde{X}_{\sigma}:=\widehat{X}_{\widetilde{\sigma}}, \quad \text { and } \quad \widetilde{X}_{\Delta^{\sigma}}:=X_{\widetilde{\Delta}^{\sigma}} \times_{X_{\tilde{\sigma}}} \widetilde{X}_{\sigma} .
$$

4. $\tilde{X}_{\sigma}$ is a $\Gamma_{\sigma}$-stratified toroidal scheme with the strata described by embedded $\Gamma_{\sigma}$-semifan $\sigma \subset \widetilde{\sigma}$. The isomorphism $\widehat{\phi}_{\sigma}$ preserves strata.

5. The morphism $\widehat{f}_{x}: Y \times_{X} \widehat{X}_{x} \rightarrow \widehat{X}_{x}$ is $\operatorname{Aut}^{\Gamma}\left(\widehat{X}_{x}, S\right)^{0}$-equivariant .

6. The morphism $\widetilde{X}_{\Delta^{\sigma}} \rightarrow \widetilde{X}_{\sigma}$ is $\operatorname{Aut}\left(\widetilde{X}_{\sigma}\right)^{0}$-equivariant.

Remark. In what follows we assume that $n$ in the definition of $\widetilde{\sigma}, \widetilde{\Delta}, \widetilde{X}_{\sigma}$, and $\widetilde{X}_{\Delta}$ denotes the dimension of relevant stratified toroidal varieties. If the semicomplexes are not associated to stratified toroidal varieties then $n$ will denote some, a priori given, natural number, satisfying the condition that all simplices and semicomplexes have dimension $\leq n$. Thus the definition of $\widetilde{\sigma}, \widetilde{\Delta}, \widetilde{X}_{\sigma}$, and $\widetilde{X}_{\Delta}$ will make sense for any cones $\sigma$ and any fans $\Sigma$ considered. 
Lemma 4.13.2. Let $\Sigma$ be an oriented $\Gamma$-semicomplex and $\sigma \in \Sigma$. Let $\phi_{1}, \phi_{2}:(U, S) \rightarrow\left(X_{\sigma}, S_{\sigma}\right)$ be smooth morphisms of $\Gamma$-stratified toroidal schemes which determine the same orientation at a closed point $x \in$ $\operatorname{strat}_{X}(\sigma)$. Let $\Delta^{\sigma}$ be a subdivision of $\sigma$ such that $\widetilde{X}_{\Delta^{\sigma}} \rightarrow \widetilde{X}_{\sigma}$ is $\operatorname{Aut}\left(\widetilde{X}_{\sigma}, S_{\sigma}\right)^{0}$-equivariant. Let $\widetilde{U}_{i}=$ $U \times_{X_{\sigma}} X_{\Delta^{\sigma}}$ denote the fiber products for $\phi_{i}$, where $i=1,2$. Then $\widetilde{U}_{i}$ are isomorphic over some neighborhood $V \subset U$ of $x \in X$.

Proof. Identical with the proof of Lemma 4.12.11.

We shall assign to the faces of an oriented $\Gamma$-semicomplex $\Sigma$ the collection of connected proalgebraic groups

$$
G^{\sigma}=\operatorname{Aut}\left(\widetilde{X}_{\sigma}\right)^{0}
$$

Definition 4.13.3. A proper subdivision $\Delta=\left\{\Delta^{\sigma} \mid \sigma \in \Sigma\right\}$ of an oriented $\Gamma$-semicomplex $\Sigma$ is called canonical if for any $\sigma \in \Sigma, G^{\sigma}$ acts on $\tilde{X}_{\Delta^{\sigma}}$ (as an abstract group) and the morphism $\tilde{X}_{\Delta^{\sigma}} \rightarrow \tilde{X}_{\sigma}$ is $G^{\sigma}$-equivariant.

Remark. This technical definition is replaced by a simple combinatorial definition by using the notion of stable support (see section 7.6). Also $\widetilde{X}_{\sigma}$ and $\widetilde{X}_{\Delta^{\sigma}}$ are replaced with $\widehat{X}_{\sigma}$ and $\widehat{X}_{\Delta^{\sigma}}$ (Proposition 7.6.2).

\subsection{Correspondence between canonical subdivisions of semicomplexes and toroidal modifica-} tions.

Theorem 4.14.1. Let $(X, S)$ be an oriented $\Gamma$-stratified toroidal variety with the associated oriented $\Gamma$ semicomplex $\Sigma$. There exists a 1-1 correspondence between the toroidal modifications $Y$ of $(X, S)$ and the canonical subdivisions $\Delta$ of $\Sigma$.

1. If $\Delta$ is a canonical subdivision of $\Sigma$ then the toroidal modification associated to it is defined locally by

$$
\begin{array}{cccc}
U_{\sigma} & \longrightarrow & X_{\sigma} \\
\uparrow f & & \uparrow \\
U_{\sigma} \times_{X_{\sigma}} X_{\Delta^{\sigma}} \simeq f^{-1}\left(U_{\sigma}\right) & \rightarrow & X_{\Delta^{\sigma}}
\end{array}
$$

2. If $Y^{1} \rightarrow X, Y^{2} \rightarrow X$ are toroidal modifications associated to canonical subdivisions $\Delta_{1}$ and $\Delta_{2}$ of $\Sigma$ then the natural birational map $Y^{1} \rightarrow Y^{2}$ is a morphism iff $\Delta_{1}$ is a subdivision of $\Delta_{2}$.

Proof. We shall construct the canonical subdivision $\Delta$ of $\Sigma$ associated to a given toroidal modification $Y$.

By Definition 4.12.1 for any point $x$ there is a chart and a subdivision $\Delta^{\sigma}$ of $\sigma$ and the fiber square stated in property (1). For two charts $\phi_{\sigma, 1}: U_{\sigma, 1} \rightarrow X_{\sigma}$ and $\phi_{\sigma, 2}: U_{\sigma, 2} \rightarrow X_{\sigma}$ we may get two subdivisions $\Delta_{1}^{\sigma}$ and $\Delta_{2}^{\sigma}$ of $\sigma$. These two charts induce by Lemma 4.13.1 isomorphisms $\widetilde{\phi}_{\sigma, i}: \widehat{X}_{x} \rightarrow \widetilde{X}_{\sigma}$ and their liftings $\widetilde{\phi}_{i}^{f}: Y \times_{X} \widehat{X}_{x} \simeq \widetilde{X}_{\Delta_{1}^{\sigma}} \simeq \widetilde{X}_{\Delta_{2}^{\sigma}}$. The isomorphisms $\widetilde{\phi}_{\sigma, i}$ define an automorphism $\alpha:=\widetilde{\phi}_{\sigma, 2} \widetilde{\phi}_{\sigma, 1}^{-1}$ of $\widetilde{X}_{\sigma}$ preserving orientation and strata, and its lifting isomorphism $\alpha_{Y}:=\widetilde{\phi}_{\sigma, 2}^{f}\left(\widetilde{\phi}_{\sigma, 1}^{f}\right)^{-1}: \widetilde{X}_{\Delta_{1}^{\sigma}} \rightarrow \widetilde{X}_{\Delta_{2}^{\sigma}}$. On the other hand $\alpha$ lifts to an automorphism $\alpha^{f}$ of $\tilde{X}_{\Delta_{1}^{\sigma}} \simeq Y \times_{X} \widehat{X}_{x}$. Finally the isomorphism $\alpha_{Y}^{\prime} \alpha_{Y}\left(\alpha^{f}\right)^{-1}: \widetilde{X}_{\Delta_{1}^{\sigma}} \rightarrow \tilde{X}_{\Delta_{2}^{\sigma}}$ is a lifting of the identity morphism on $\widetilde{X}_{\sigma}$. Thus it is an identity morphism on $\tilde{X}_{\Delta_{1}^{\sigma}}=\widetilde{X}_{\Delta_{2}^{\sigma}}$ and $\Delta_{1}^{\sigma}=\Delta_{2}^{\sigma}$. This implies the uniqueness of $\Delta^{\sigma}$. By Lemma 4.13.1 $(6), i_{x}: \widetilde{X}_{\Delta^{\sigma}} \rightarrow \widetilde{X}_{\sigma}$ is $\operatorname{Aut}\left(\widetilde{X}_{\sigma}\right)^{0}$-equivariant. Thus the fan $\Delta^{\sigma}$ is $\operatorname{Aut}(\sigma)^{0}$-invariant. It follows from the uniqueness that if $\tau \leq \sigma$ then $\Delta^{\sigma} \mid \tau=\imath_{\tau}^{\sigma}\left(\Delta^{\tau}\right) \simeq \Delta^{\tau}$. Therefore the subdivisions $\Delta^{\sigma}$ define the canonical subdivision $\Delta$ of $\Sigma$.

Now let $\Delta$ be a canonical subdivision of the oriented $\Gamma$-semicomplex $\Sigma$. We shall construct the toroidal modification of $(X, S)$ associated to $\Delta$. For any chart $U_{\sigma, a} \rightarrow X_{\sigma}$ set

$$
\widetilde{U}_{\sigma, a}:=U_{\sigma, a} \times_{X_{\sigma}} X_{\Delta \sigma}
$$

For any two charts $\phi_{\sigma, a}: U_{\sigma, a} \rightarrow X_{\sigma}$ and $\phi_{\tau, b}: U_{\tau, b} \rightarrow X_{\tau}$ set

$$
\widetilde{U}_{\sigma, \tau, a, b}:=\left(U_{\sigma, a} \cap U_{\tau, b}\right) \times_{X_{\sigma}} X_{\Delta^{\sigma}},
$$

where the fiber product is defined for the restriction of $\phi_{\sigma, a}: U_{s, a} \rightarrow X_{\sigma}$ to $U_{\sigma, a} \cap U_{\tau, b}$.

It follows from Lemma 4.12.11 that $\widetilde{U}_{\sigma, \tau, a, b}$ is isomorphic over $U_{\sigma, a} \cap U_{\tau, b}$ to $\widetilde{U}_{\tau, \sigma, b, a}$. Thus we can glue all the sets $\widetilde{U}_{\sigma, a}$ along $\widetilde{U}_{\sigma, \tau, a, b}$ and get a separated variety $Y$. Note that the action of $\Gamma$ lifts to any subset $\widetilde{U}_{\sigma, a}$ and hence to an action on the whole $Y$. 
A chart $\phi_{\sigma}: U_{\sigma} \rightarrow X_{\sigma}$ defines a $\Gamma_{\sigma}$-equivariant isomorphism $\widehat{X}_{x} \stackrel{\alpha_{7}}{\rightarrow} \widetilde{X}_{\sigma}$. For any $x \in \operatorname{strat}_{X}(\sigma)$ any automorphism $\alpha$ of $\widehat{X}_{x} \simeq \widetilde{X}_{\sigma}$ preserving strata and orientation can be lifted to a $\Gamma_{\sigma}$-equivariant automorphism $\alpha^{\prime}$ of $\widehat{X}_{y} \times_{X} Y \simeq \widetilde{X}_{\Delta^{\sigma}}$.

(1) Follows from the construction of a toroidal modification from a canonical subdivision.

(2) Let $Y^{1} \rightarrow X, Y^{2} \rightarrow X$ be toroidal modifications associated to canonical subdivisions $\Delta_{1}$ and $\Delta_{2}$. If $\Delta_{1}$ is a subdivision of $\Delta_{2}$ then the natural birational map $Y^{1} \rightarrow Y^{2}$ is a morphism since it is a morphism for each chart. On the other hand if $Y^{1} \rightarrow Y^{2}$ is a morphism then $Y^{1} \times_{X} \widehat{X}_{x} \simeq \widetilde{X}_{\Delta_{1}^{\sigma}} \rightarrow Y^{2} \times_{X} \widehat{X}_{x} \simeq \widetilde{X}_{\Delta_{2}^{\sigma}}$ is a toric morphism. By Lemma 1.12.9 the latter morphism is induced by a toric morphism $X_{\Delta_{1}^{\sigma}} \rightarrow X_{\Delta_{2}^{\sigma}}$. Hence $\Delta_{1}^{\sigma}$ is a subdivision of $\Delta_{2}^{\sigma}$ for each $\sigma \in \Sigma$ and consequently $\Delta_{1}$ is a subdivision of $\Delta_{2}$. This completes the proof of Theorem 4.14.1.

\section{Stable valuations}

For simplicity we consider only valuations with integral values.

\subsection{Monomial valuations.}

Definition 5.1.1. Let $R$ be a local noetherian ring, $u_{1}, \ldots, u_{k}$ be generators of its maximal ideal, and let $a_{1}, \ldots, a_{k}$ be nonnegative integers. For any $a \in \mathbf{Z}$ set

$$
J_{a}:=\left(u^{i_{1}, \ldots, i_{k}} \mid i_{1} a_{1}+\ldots+i_{k} a_{k} \geq a\right) \subset R,
$$

where $i_{1}, \ldots, i_{k}$, is a multiindex in $\mathbf{Z}_{\geq 0}^{k}$. We call a valuation $\nu$ monomial with basis $u_{1}, \ldots, u_{k}$ and weights $a_{1}, \ldots, a_{k}$ if for any $f \in R \backslash\{0\}$,

$$
\nu(f)=\max \left\{a \in \mathbf{Z} \mid f \in J_{a}\right\} .
$$

Lemma 5.1.2. Let $\nu$ be a nonnegative monomial valuation of a local noetherian ring $R$. Then for any $a \in \mathbf{Z}, I_{\nu, a}=J_{a}$.

Lemma 5.1.3. Let $\nu$ be a nonnegative valuation of a local noetherian ring $R$, and $u_{1}, \ldots, u_{k}$ be generators of the maximal ideal of $R$. Let $\nu_{0}$ be a monomial valuation with basis $u_{1}, \ldots, u_{k}$ such that $\nu_{0}\left(u_{i}\right)=\nu\left(u_{i}\right) \geq 0$. Then $\nu_{0}(f) \leq \nu(f)$ for any $f \in R$.

Proof. $I_{\nu_{0}, a}:=\left\{f \in R \mid \nu_{0}(f) \geq a\right\}=J_{a} \subset I_{\nu, a}$.

Lemma 5.1.4. Let $\nu$ be a nonnegative monomial valuation of a local noetherian ring $R$ with basis $u_{1}, \ldots, u_{k}$. Let $g$ be an automorphism of $R$ such that $\nu\left(g\left(u_{i}\right)\right) \geq \nu\left(u_{i}\right)$ for any $i=1, \ldots, k$. Then $\nu(g(f))=\nu\left(u_{i}\right)$ for any $f \in R$.

Proof. Note that $\nu\left(g\left(u^{i_{1}, \ldots, i_{k}}\right)\right) \geq \nu\left(g\left(u^{i_{1}, \ldots, i_{k}}\right)\right)$. By Lemma 5.1.2, $g\left(I_{\nu, a}\right)=g\left(J_{a}\right) \subset I_{\nu, a}$. If $g\left(I_{\nu, a}\right) \subset \neq I_{\nu, a}$ then we obtain an infinite ascending chain of ideals $I_{\nu, a} \subset g^{-1}\left(I_{\nu, a}\right) \subset g^{-2}\left(I_{\nu, a}\right) \subset \ldots$

5.2. Toric and locally toric valuations. Let $X$ be an algebraic variety and $\nu$ be a valuation of the field $K(X)$ of rational functions. By the valuative criterion of separatedness and properness the valuation ring of $\nu$ dominates the local ring of a uniquely determined point (in general nonclosed) $c_{\nu}$ on a complete variety $X$. (If $X$ is not complete such a point may not exist). We call the closure of $c_{\nu}$ the center of the valuation $\nu$ and denote it by $\mathrm{Z}(\nu)$ or $\mathrm{Z}(\nu, X)$. For any $x \in \mathrm{Z}(\nu)$ and $a \in \mathbf{Z}_{\geq 0}$,

$$
I_{\nu, a, x}:=\left\{f \in \mathcal{O}_{X, x} \mid \nu(f) \geq a\right\}
$$

is an ideal in $\mathcal{O}_{X, x}$. For fixed $a$ these ideals define a coherent sheaf of ideals $\mathcal{I}_{\nu, a}$ supported at $\mathrm{Z}(\nu)$.

Lemma 5.2.1. Let $v$ be an integral vector in the support of the fan $\Sigma$. Then the toric valuation $\operatorname{val}(v)$ is centered on $\bar{O}_{\sigma}$, where $\sigma$ is the cone whose relative interior contains $v$.

Proof. Let $I_{O_{\sigma}} \subset K\left[X_{\sigma}\right]$ be the ideal of the orbit $O_{\sigma}$. Let $f=\sum a_{i} x^{m_{i}}, m_{i} \in \sigma^{\vee}$, be a regular function on $X_{\sigma}$. Then $\operatorname{val}(v)(f)>0$ iff $\left(v, m_{i}\right)>0$ for all $a_{i} \neq 0$. But $\left(v, m_{i}\right) \geq 0$ by definition and $\left(v, m_{i}\right)=0$ iff $m_{i} \in \sigma^{\perp}$. Thus we have $\left(v, m_{i}\right)>0$ iff $m_{i} \in \sigma^{\vee} \backslash \sigma^{\perp}$. Finally $I_{O_{\sigma}}=\left\{f \in K\left[X_{\sigma}\right] \mid \operatorname{val}(v)(f)>0\right\}$ and the valuation ring dominates the local ring of $O_{\sigma}$. 
Let $L$ be a ring containing $K, \sigma$ be a cone of the maximal dimension in $N_{\sigma}$ and $v$ be an integral vector of $\sigma$. Toric valuation $\operatorname{val}(v)$ can be defined on any $\operatorname{ring} L\left[\left[\sigma^{\vee}\right]\right]$ and its arbitrary subring $R$ containing $L\left[\sigma^{\vee}\right]$. For any $f=\sum a_{i} x^{m_{i}}$, set

$$
\operatorname{val}(v)(f)=\min \left\{\left(m_{i}, v\right) \mid a_{i} \neq 0\right\} .
$$

The valuation $\operatorname{val}(v)$ of $R$ will be sometimes denoted by $\operatorname{val}(v, R)$ or $\operatorname{val}(v, \operatorname{Spec}(R))$.

Lemma 5.2.2. Let $\sigma$ be a cone of a maximal dimension and $T_{\sigma}$ denote the "big torus" acting on $\widehat{X}_{\sigma}$. Let $\mu$ be a $T_{\sigma}$-invariant valuation on $\widehat{X}_{\sigma}$. Then there exists an integral vector $v \in \sigma$ such that $\operatorname{val}(v)=\mu$.

Proof. The valuation $\mu$ defines a linear function on $\sigma^{\vee}$ corresponding to an integral vector $v \in \sigma$. Since $\mu$ is $T_{\sigma}$ invariant so are the ideals $I_{\mu, a}$. Therefore the ideals $I_{\mu, a}$ are generated by characters $x^{m}$, where $(m, v) \geq a$. Consequently, $I_{\mu, a}=I_{\operatorname{val}(v), a}$ and $\mu=\operatorname{val}(v)$.

Lemma 5.2.3. Let $\sigma$ be a cone of the maximal dimension in a lattice $N_{\sigma}$ and $v \in \sigma$ be its integral vector. Let $R$ be a subring of $K\left[\widehat{X}_{\sigma}\right]$ that contains $K\left[X_{\sigma}\right]$. Then for any $a \in \mathbf{Z}$ we have $I_{\operatorname{val}\left(v, X_{\sigma}\right), a} \cdot R=I_{\operatorname{val}(v, R), a}$

Proof. Denote by $R^{\prime}$ the localization of $R$ at the maximal ideal of $O_{\sigma}$. Then $I_{\operatorname{val}\left(v, R^{\prime}\right), a}=I_{\mathrm{val}\left(v, \widehat{X}_{\sigma}\right), a} \cap R^{\prime}=$ $I_{\mathrm{val}\left(v, X_{\sigma}\right), a} \cdot K\left[\widehat{X}_{\sigma}\right] \cap R^{\prime}$. Since $K\left[\widehat{X}_{\sigma}\right]$ is faithfully flat over $R^{\prime}$ we obtain $I_{\operatorname{val}\left(v, X_{\sigma}\right), a} \cdot K\left[\widehat{X}_{\sigma}\right] \cap R^{\prime}=I_{\mathrm{val}\left(v, X_{\sigma}\right), a}$. $R^{\prime}$. Thus we have $I_{\operatorname{val}\left(v, R^{\prime}\right), a}=I_{\operatorname{val}\left(v, X_{\sigma}\right), a} \cdot R^{\prime}$. Then $I_{\operatorname{val}(v, R), a}=I_{\operatorname{val}\left(v, R^{\prime}\right), a} \cap R=I_{\operatorname{val}\left(v, X_{\sigma}\right), a} \cdot R^{\prime} \cap R=$ $I_{\mathrm{val}\left(v, X_{\sigma}\right), a} \cdot R$.

Lemma 5.2.4. Let $X_{\Sigma}$ be a toric variety with a toric action of $\Gamma$. Let $f: U \rightarrow X_{\Sigma}$ be a smooth $\Gamma$ equivariant morphism. Let $v \in \operatorname{int}(\sigma)$, where $\sigma \in \Sigma$, be an integral vector. Assume that the inverse image of $\bar{O}_{\sigma}$ is irreducible. Then there exists a $\Gamma$-invariant valuation $\mu$ on $U$, such that $\mathcal{I}_{\mu, a}=f^{-1}\left(\mathcal{I}_{\operatorname{val}(v), a}\right) \cdot \mathcal{O}_{U}$.

Proof. Let $x$ belong to the $\operatorname{support} \operatorname{supp}\left(f^{-1}\left(\mathcal{I}_{\mathrm{val}(v), a}\right) \cdot \mathcal{O}_{U}\right)=f^{-1}\left(\bar{O}_{\sigma}\right)$. Then $f(x) \in O_{\tau}$, where $\tau \geq \sigma$. Let $\pi: X_{\tau} \rightarrow X_{\underline{\tau}}$ denote the natural projection. Then $\mathcal{I}_{\operatorname{val}\left(v, X_{\tau}\right), a}=\pi^{-1}\left(\mathcal{I}_{\operatorname{val}\left(v, X_{\underline{\tau}}, a\right.}\right) \cdot \mathcal{O}_{X_{\tau}}$. Thus it suffices to prove the lemma replacing $X_{\Sigma}$ with $X_{\underline{\tau}}$ and $U$ with the inverse image $U^{\prime}$ of $X_{\tau}^{-}$. Let $U_{x} \subset U^{\prime}$ be an open neighborhood of $x$ for which there exists an étale extension $\tilde{f}: U_{x} \rightarrow X_{\underline{\tau} \times \operatorname{reg}(\underline{\tau})}$. It defines an isomorphism $\widehat{f}: \widehat{X}_{x} \simeq \widehat{X}_{\underline{\tau} \times \operatorname{reg}(\underline{\tau})}$. The toric valuation $\operatorname{val}\left(v, X_{\underline{\tau}}\right)$ defines the toric valuation $\operatorname{val}\left(v, X_{\underline{\tau} \times \operatorname{reg}(\underline{\tau})}\right)$ and the corresponding valuation $\bar{\mu}_{x}$ on $\widehat{X}_{x}$. By Lemma 5.2.3, $\bar{\mu}_{x}$ determines the valuation $\mu_{x}$ of $\mathcal{O}_{X, x}$ such that $\left(\mathcal{I}_{\mu_{x}, a}\right)_{x}=f^{-1}\left(\mathcal{I}_{\operatorname{val}(v), a}\right) \cdot \mathcal{O}_{X, x}$. Thus $\mu_{x}$ is a valuation on $U_{x}$ supported on the irreducible set $\overline{f^{-1}\left(O_{\sigma}\right)}$. Note that both sheaves of ideals $\mathcal{I}_{\mu_{x}, a}$ and $f^{-1}\left(\mathcal{I}_{\mathrm{val}(v), a}\right) \cdot \mathcal{O}_{X}$ are equal in some open neighborhood $V_{x}$ of $x$. Therefore the valuation $\mu_{y}$ is the same for all closed point $y \in \overline{f^{-1}\left(O_{\sigma}\right)} \cap V_{x}$. Since $\overline{f^{-1}\left(O_{\sigma}\right)}$ is irredducibe the valuations $\mu_{x}$ are the same for all $x \in \overline{f^{-1}\left(O_{\sigma}\right)}$ and define a unique valuation $\mu$. Since the sheaves of ideals $f^{-1}\left(\mathcal{I}_{\operatorname{val}(v), a}\right) \cdot \mathcal{O}_{X}=\mathcal{I}_{\mu, a}$ are $\Gamma$-invariant $\mu$ is also $\Gamma$-invariant.

Let $f: X \rightarrow Y$ be a dominant morphism and $\nu$ be a valuation of $K(X)$. Then $f_{*}(\nu)$ denotes the valuation which is the restriction of $\nu$ to $K(Y) \simeq f^{*}(K(Y)) \subset K(X)$.

Let $\nu$ be a valuation on $X$ and $\mathcal{I}_{\nu, a}$ be the associated sheaves of ideals. Let $f: Y \rightarrow X$ be a morphism for which $f^{-1}\left(\mathcal{I}_{\nu, a}\right) \cdot \mathcal{O}_{Y}$ determine a unique valuation $\mu$ on $Y$ such that for sufficiently divisible integer $a$, $\mathcal{I}_{\mu, a}=f^{-1}\left(\mathcal{I}_{\nu, a}\right) \cdot \mathcal{O}_{Y}$. We denote this valuation by $\mu=f^{*}(\nu)$.

Definition 5.2.5. By a locally toric valuation on an $\Gamma$-toroidal variety $X$ we mean a valuation $\nu$ of $K(X)$ such that for any point $x \in \mathrm{Z}(\nu, X)$ there exists a $\Gamma$-equivarinat $\Gamma_{x}$-smooth morphism $\phi: U \rightarrow X_{\sigma}$ from an open $\Gamma$-invariant neighborhood $U$ of $x$ to a toric variety $X_{\sigma}$ with a toric action of $\Gamma \operatorname{such}$ that $\mu=\phi^{*}(\operatorname{val}(v))$, where $v$ is an integral vector of $\sigma$.

If $\mu$ is a locally toric valuation on $X$ then by $\mu_{\mid \widehat{X}_{x}}$ we denote the induced valuation on $\widehat{X}_{x} \simeq \widehat{X}_{\sigma}$.

Definition 5.2.6. By the blow-up $\mathrm{bl}_{\nu}(X)$ of $X$ at a locally toric valuation $\nu$ we mean the normalization of

$$
\operatorname{Proj}\left(\mathcal{O} \oplus \mathcal{I}_{\nu, 1} \oplus \mathcal{I}_{\nu, 2} \oplus \ldots\right),
$$

Lemma 5.2.7. For any natural $l, \operatorname{Proj}\left(\mathcal{O} \oplus \mathcal{I}_{\nu, 1} \oplus \mathcal{I}_{\nu, 2} \oplus \ldots\right)=\operatorname{Proj}\left(\mathcal{O} \oplus \mathcal{I}_{\nu, l} \oplus \mathcal{I}_{\nu, 2 l} \oplus \ldots\right)$.

Denote by $\mathrm{bl}_{\mathcal{J}}(X) \rightarrow X$ the blow-up of any coherent sheaf of ideals $\mathcal{J}$. 
Lemma 5.2.8. Let $X_{\Sigma}$ be a toric variety associated to a fan $\Sigma$ in $N$ and $v \in|\Sigma| \cap N$. Then $\operatorname{bl}_{\operatorname{val}(v)}\left(X_{\Sigma}\right)$ is the toric variety associated to the subdivision $\langle v\rangle \cdot \Sigma$. Moreover for any sufficiently divisible integer $d$, $\mathrm{bl}_{\mathrm{val}(v)}\left(X_{\Sigma}\right)$ is the normalization of the blow-up of $\mathcal{I}_{\nu, d}$.

Proof. It follows from Definition 5.2.6 that $\operatorname{bl}_{\mathrm{val}(v)}\left(X_{\Sigma}\right)$ is a normal toric variety. By Lemma 5.2.1, the sheaf of ideals $\mathcal{I}_{\mathrm{val}(v), d}$ is supported on $\bar{O}_{\sigma}$, where $v \in \operatorname{int}(\sigma), \sigma \in \Sigma$. By [39], Ch.I, Th. 9, it determines a convex piecewise linear function $f:=\operatorname{ord}_{\mathcal{I}_{\mathrm{val}(v), d}}:|\Sigma| \rightarrow \mathbf{R}$ such that that $f=0$ on any $\tau \in \Sigma$ that does not contain $v$, and $f(u)=\min \left\{(m, u) \mid m \in \tau^{\vee} \cap M,(m, v) \geq d\right\}$ for any $\tau$ containing $v$. Assume that $\tau$ contains $v$ and let $\tau^{\prime}$ be a face of $\tau$ that does not contain $v$. Let $m_{\tau^{\prime}, \tau} \in \tau^{\vee}$ be a nonnegative integral functional on $\tau$ which equals 0 exactly on $\tau^{\prime}$. Assume that $d$ divides all $\left(m_{\tau^{\prime}, \tau}, v\right)$. Then $f_{\mid \tau^{\prime}+\langle v\rangle}=\frac{d}{\left(m_{\tau^{\prime}, \tau}, v\right)} \cdot m_{\tau^{\prime}, \tau}$. Consequently, $\mathcal{I}_{\operatorname{val}(v), d}^{k}=\mathcal{I}_{\mathrm{val}(v), k d}$ and $\mathrm{bl}_{\mathrm{val}(v)}\left(X_{\Sigma}\right)=\mathrm{bl}_{\mathcal{I}_{\mathrm{val}(v), d}}\left(X_{\Sigma}\right)$. By 39] Ch II Th. 10, the blow-up of $\mathcal{I}_{\mathrm{val}(v), d}$ corresponds to the minimal subdivision $\Sigma^{\prime}$ of $\Sigma$ such that $f$ is linear on each cone in $\Sigma^{\prime}$. By the above,

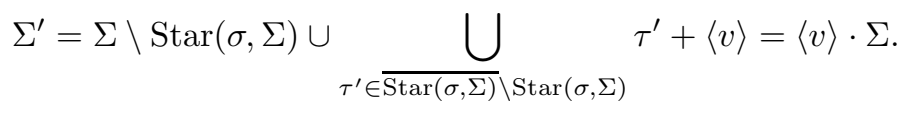

Proposition 5.2.9. For any locally toric valuation $\nu$ on $X$ there exists an integer $d$ such that

- $\mathrm{bl}_{\nu}(X)=\mathrm{bl}_{\mathcal{I}_{\nu, d}}(X)$.

- The valuation $\nu$ is induced by an irreducible $Q$-Cartier divisor on $\mathrm{bl}_{\nu}(X)$.

Proof. By quasicompactness of $X$ one can find a finite open affine covering $U_{i}$ of $X$ of charts $\phi_{i}: U_{i} \rightarrow X_{\sigma_{i}}$ such that the valuation $\nu$ on each $U_{i} \subset X$ is equal to $\phi_{i}^{*}\left(\operatorname{val}\left(v_{i}\right)\right.$. It follows from Lemma 5.2.8 that for any $i$ there exists $d_{i}$ that $\mathrm{bl}_{\nu}\left(U_{i}\right)=\mathrm{bl}_{\mathcal{I}_{\nu, d_{i}}}\left(U_{i}\right)$. It suffices to take $d$ equal the product of all $d_{i}$.

\subsection{Stable vectors and stable valuations.}

Definition 5.3.1. Let $(X, S)$ be an oriented $\Gamma$-stratified toroidal variety. A locally toric $\Gamma$-invariant valuation $\nu$ is stable on $X$ if

1. $\mathrm{Z}(\nu, X)=\bar{s}$ for some stratum $s \in S$.

2. For any $x \in \bar{s}, \nu_{\mid \widehat{X}_{x}}$ is invariant with respect to any $\Gamma_{s}$-equivariant automorphism of $\left(\widehat{X}_{x}, S\right)$, preserving orientation.

Let $f: Y \rightarrow(X, S)$ be a toroidal modification of an oriented $\Gamma$-stratified toroidal variety. Then a valuation $\nu$ on $Y$ is called $X$-stable if $f_{*}(\nu)$ is stable on $(X, S)$.

Definition 5.3.2. Let $\Sigma$ be an oriented $\Gamma$-semicomplex. A vector $v_{0} \in \operatorname{int}\left(\sigma_{0}\right)$, where $\sigma_{0} \in \Sigma$, is $\Sigma$-stable on $\Sigma$ if for any $\tau \geq \sigma_{0}$ the corresponding valuation $\operatorname{val}\left(\imath_{\sigma_{0}}^{\tau}(v)\right)$ on $\widetilde{X}_{\tau}$ is $G^{\tau}$-invariant. A vector $v \in \sigma$, where $\sigma \in \Sigma$ is $\Sigma$-stable if there is a stable vector $v_{0} \in \operatorname{int}\left(\sigma_{0}\right)$, where $\sigma_{0} \leq \sigma$, for which $v=\imath_{\sigma_{0}}^{\sigma}\left(v_{0}\right)$.

Let $\Sigma$ be an oriented $\Gamma$-semicomplex. For any $\sigma \in \Sigma$ set

$$
\operatorname{stab}(\sigma):=\left\{a \cdot v \mid v \in \sigma, \quad v \text { is a stable vector }, a \in \mathbf{Q}_{\geq 0}\right\} .
$$

Lemma 5.3.3. 1. Stable vectors $v \in \sigma$ are Aut $(\sigma)^{0}$-invariant.

2. For any $\varrho \geq \tau \geq \sigma$ and any vector $v \in \operatorname{stab}(\sigma), \imath_{\tau}^{\varrho} \tau_{\sigma}^{\tau}(v)=\imath_{\sigma}^{\varrho}(v)$.

3. $\imath_{\sigma}^{\tau}(\operatorname{stab}(\sigma))=\imath_{\sigma}^{\tau}(\sigma) \cap \operatorname{stab}(\tau)$ for any $\tau \geq \sigma$.

Proof.(1) Follows from definition.

(2) By definition there is $v_{0} \in \operatorname{int}\left(\sigma_{0}\right)$ for which $v=\imath_{\sigma_{0}}^{\sigma}\left(v_{0}\right)$. First we prove that for any $\tau \geq \sigma$, we have $\imath_{\sigma}^{\tau} \imath_{\sigma_{0}}^{\sigma}\left(v_{0}\right)=\imath_{\sigma_{0}}^{\tau}\left(v_{0}\right)$.

By Definition 4.11 .1 there is an automorphism $\alpha_{0} \in \operatorname{Aut}\left(\sigma_{0}\right)^{0}$ which preserves the orientation such that $\imath_{\sigma}^{\tau} \imath_{\sigma_{0}}^{\sigma}=\imath_{\sigma_{0}}^{\tau} \alpha_{0}$. By Definition 5.3 .2 the valuation $\operatorname{val}\left(v_{0}\right)$ is $G^{\sigma_{0}}$-invariant on $\widetilde{X}_{\sigma_{0}}$ and hence $v_{0}$ is preserved by $\alpha_{0}$. This gives $\imath_{\sigma}^{\tau} l_{\sigma_{0}}^{\sigma}\left(v_{0}\right)=\imath_{\sigma_{0}}^{\tau} \alpha_{0}\left(v_{0}\right)=\imath_{\sigma_{0}}^{\tau}\left(v_{0}\right)$. Now let $\varrho \geq \tau \geq \sigma$. By the above we have $\imath_{\sigma}^{\varrho} \imath_{\sigma_{0}}^{\sigma}\left(v_{0}\right)=\imath_{\sigma_{0}}\left(v_{0}\right)$ Then $\imath_{\tau}^{\varrho} \tau_{\sigma}^{\tau}(v)=\imath_{\tau}^{\varrho} \imath_{\sigma_{0}}^{\tau}\left(v_{0}\right)=\imath_{\sigma_{0}}^{\varrho}\left(v_{0}\right)$.

Finally $\imath_{\sigma_{0}}^{\varrho}\left(v_{0}\right)=\imath_{\sigma}^{\varrho} \sigma_{\sigma_{0}}^{\sigma}\left(v_{0}\right)=\imath_{\sigma}^{\varrho}(v)$. 
(3) If $v$ is a stable vector on $\sigma$ then by Definition 5.3.2 there is $\sigma_{0} \leq \sigma$ and a stable vector $v_{0} \in \operatorname{int}\left(\sigma_{0}\right)$ such that $v=\imath_{\sigma_{0}}^{\sigma}\left(v_{0}\right)$. Then for $\tau \geq \sigma, \imath_{\sigma_{0}}^{\tau}\left(v_{0}\right)=\imath_{\sigma}^{\tau} \imath_{\sigma_{0}}^{\sigma}\left(v_{0}\right)=\imath_{\sigma}^{\tau}(v)$ is stable on $\tau$. If $w \in \imath_{\sigma}^{\tau}(\sigma) \cap \operatorname{stab}(\tau)$ then there is $\sigma_{0} \leq \sigma$ and a stable vector $v_{0} \in \operatorname{int}\left(\sigma_{0}\right)$ such that $w=\imath_{\sigma_{0}}^{\tau}\left(v_{0}\right)=\imath_{\sigma}^{\tau} \imath_{\sigma_{0}}^{\sigma}\left(v_{0}\right)=\imath_{\sigma}^{\tau}(v)$, where $v=\imath_{\sigma_{0}}^{\sigma}\left(v_{0}\right)$.

Example 5.3.4. Let $\Sigma$ be a regular semicomplex and $\sigma=\left\langle e_{1}, \ldots, e_{k}\right\rangle \in \Sigma$. Then $e:=e_{1}+\ldots+e_{k}$ is stable since it corresponds to the valuation of the stratum $\operatorname{strat}_{X}(\sigma)$ on $\widetilde{X}_{\sigma}$. The subset $s$ defines a smooth subvariety in a smooth neighborhood and therefore it determines a valuation. If we blow-up the subvariety $\operatorname{strat}_{X}(\sigma)$ then this valuation coincides with the one defined by the exceptional divisor.

Example 5.3.5. Consider the stratified toroidal variety $\mathbf{A}^{2}$ from Example 3.1.13. The valuation of the point $(0,0)$ corresponds to the vector $(1,1) \in N$, the valuation of $\bar{s}=\mathbf{A}^{1} \times\{0\}$ corresponds to the vector $(0,1) \in N$. Then $|\operatorname{Stab}(\Sigma)|=\langle(1,1),(0,1)\rangle$. The valuations from $\langle(1,0),(0,1)\rangle \backslash\langle(1,1),(0,1)\rangle$ are not stable: Let $\widetilde{\mathbf{A}^{2}} \rightarrow \mathbf{A}^{2}$ be the blow-up of the point $(0,0) \in \mathbf{A}^{2}$. The valuations from $\langle(1,0),(0,1)\rangle\langle(1,1),(0,1)\rangle$ are centered on $\widetilde{\mathbf{A}^{2}}$ at $l_{2} \cap D$ or $l_{2}$ (using notation from Example 3.1.13) and the automorphism $\phi: \mathbf{A}^{2} \rightarrow \mathbf{A}^{2}$, $\phi\left(x_{1}, x_{2}\right)=\left(x_{1}+x_{2}, x_{2}\right)$, preserves strata and moves the subvarieties $l_{2}$ and $l_{2} \cap D$. In particular it changes the relevant valuations.

Lemma 5.3.6. Let $(X, S)$ be a $\Gamma$-stratified toroidal variety with the associated oriented $\Gamma$-semicomplex $\Sigma$. The following conditions are equivalent:

1. $\nu$ is stable on $X$.

2. There exists a stable vector $v \in \operatorname{int}(\sigma)$, where $\sigma \in \Sigma$, such that for any $\tau \geq \sigma$ and any chart $\phi: U \rightarrow X_{\tau}$ we have $\nu=\phi^{*}\left(\operatorname{val}\left(i_{\sigma}^{\tau}(v)\right)\right)$.

Proof. Let $x$ denote a closed point of $\overline{\operatorname{strat}_{X}(\sigma)}$. Then $x \in \operatorname{strat}_{X}(\tau)$, where $\tau \geq \sigma$. The valuation $\nu$ on $X$ determines a $G^{\tau}$-invariant valuation on $\widehat{X}_{x} \simeq \widetilde{X}_{\tau}$ which is in particular a toric valuation so, by Lemmas 5.2 .2 and 5.2.1 it corresponds to a unique $v \in \operatorname{int}(\sigma)$. Thus $v$ is stable and ,by Lemma 5.2.4, $\nu=\phi^{*}(\operatorname{val}(v))$.

Let $v$ be a stable vector. The valuation $\operatorname{val}(v)$ determines a monomial valuation $\nu:=\widehat{\phi}^{*}(\operatorname{val}(v))$ on $\widehat{X}_{x}$, where $x \in \overline{\operatorname{strat}_{X}(\sigma)} \cap U$. Since $\operatorname{val}(v)$ is $G^{\tau}$-invariant on $\tilde{X}_{\tau}$ for any $\tau \geq \sigma$ we see that $\nu_{\mid \widehat{X}_{x}}$ is invariant with respect to any $\Gamma_{x}$-equivariant automorphism preserving strata and orientation. Moreover $\nu_{\mid \widehat{X}_{x}}$ and $\nu$ do not depend on $\phi$.

Lemma 5.3.7. Let $f: Y \rightarrow X$ be a toroidal modification of a $\Gamma$-stratified toroidal variety $(X, S)$ associated to a canonical subdivision $\Delta$ of $\Sigma$. The following conditions are equivalent:

1. $\nu$ is $X$-stable on $Y$.

2. There exists a $\Sigma$-stable vector $v \in \operatorname{int}(\sigma)$, where $\sigma \in \Sigma$, such that for any $\tau \geq \sigma$ and morphism $\phi_{\tau}^{f}: f^{-1}\left(U_{\tau}\right) \rightarrow X_{\Delta^{\tau}}$, induced by a chart $\phi_{\tau}: U_{\tau} \rightarrow X_{\tau}$ we have $\nu=\phi_{\tau}^{f *}(\operatorname{val}(v))$.

Proof. Let $y \in \mathrm{Z}(\nu, Y), x=f(y) \in X$ and $\phi: U \rightarrow X_{\sigma}$ be a chart at $x$. We can extend this $\Gamma^{\sigma}$-smooth morphism to an étale morphism $\widetilde{\phi}: U \rightarrow X_{\widetilde{\sigma}}$. By Lemma 5.3.6 we have $\nu=\widetilde{\phi}^{*}(\operatorname{val}(v))$ for some stable vector $v \in \sigma$.

Consider the fiber squares

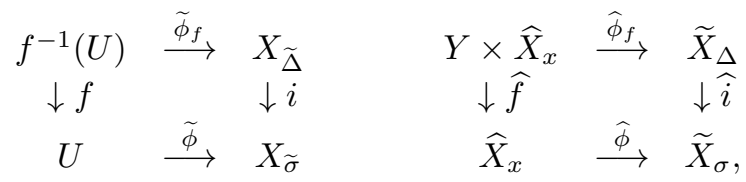

where $\widetilde{\phi}_{\sigma}^{f}$ is the induced étale morphism and $i$ is a toric morphism. Then $\widehat{\phi}$ as well as $\widehat{\phi_{\sigma}^{f}}$ are isomorphisms and we have $\nu=\widehat{f}_{*}^{-1} \widehat{\phi}^{*} i_{*}(\operatorname{val}(v))=\widehat{\phi}_{f}^{*}(\operatorname{val}(v))$ on $Y \times_{X} \widehat{X}_{x}$. Consequently $\nu=f_{*}^{-1} \widetilde{\phi}^{*} i_{*}(\operatorname{val}(v))=\widetilde{\phi}_{f}^{*}(\operatorname{val}(v))$ since the above valuations coincide on $f^{-1}(U)$.

Lemma 5.3.8. Let $\sigma$ and $\tau$ be $\Gamma$-semicones in isomorphic lattices $N_{\sigma} \simeq N_{\tau}$, and $L$ be a field containing $K$. Consider the induced action of $\Gamma$ on $L\left[\left[\sigma^{\vee}\right]\right]$ and $L\left[\left[\tau^{\vee}\right]\right]$ which is trivial on L. Let $\psi: L\left[\left[\sigma^{\vee}\right]\right] \simeq L\left[\left[\tau^{\vee}\right]\right]$ be a $\Gamma$-equivariant isomorphism over $K$. Let $v_{\sigma} \in \operatorname{int}(\sigma)$ be a minimal internal vector of $\sigma$. Then there exists a minimal internal vector $v_{\tau} \in \operatorname{int}(\tau)$ such that $\psi_{*}\left(\operatorname{val}\left(v_{\sigma}\right)\right)=\operatorname{val}\left(v_{\tau}\right)$. 
Proof. Note that $\operatorname{val}\left(v_{\sigma}\right)\left(f_{\sigma}\right)>0$ and $\psi_{*}\left(\operatorname{val}\left(v_{\sigma}\right)\right)\left(f_{\tau}\right)>0$, for any functions $f_{\sigma} \in m_{\sigma}, f_{\tau} \in m_{\tau}$ from the maximal ideals $m_{\sigma} \subset L\left[\left[\sigma^{\vee}\right]\right]$ and $m_{\tau} \subset L\left[\left[\tau^{\vee}\right]\right]$ respectively. The dual cone $\tau^{\vee}$ of regular characters defines a subgroup in the multiplicative group of rational functions. The valuation $\psi_{*}\left(\operatorname{val}\left(v_{\sigma}\right)\right)$ determines a group homomorphism of that subgroup into $\mathbf{Z}$ and hence defines a linear function on $\tau^{\vee}$ corresponding to a vector $v_{\tau} \in \operatorname{int}(\tau)$. We have to show that $\operatorname{val}\left(v_{\tau}\right)=\psi_{*}\left(\operatorname{val}\left(v_{\sigma}\right)\right)$ (it is not clear whether $\psi_{*}\left(\operatorname{val}\left(v_{\sigma}\right)\right)$ is monomial with respect to characters). By Lemma 5.1.3, $\psi_{*}\left(\operatorname{val}\left(v_{\sigma}\right)\right) \geq \operatorname{val}\left(v_{\tau}\right)$. Let $v_{\sigma}^{\prime} \in \operatorname{int}(\sigma)$ correspond to the linear function determined by $\psi^{-1 *}\left(\operatorname{val}\left(v_{\tau}\right)\right)$ on the cone $\sigma^{\vee}$. Then $\operatorname{val}\left(v_{\sigma}\right)=\psi^{-1 *} \psi_{*}\left(\operatorname{val}\left(v_{\sigma}\right)\right) \geq$ $\psi^{-1 *}\left(\operatorname{val}\left(v_{\tau}\right)\right) \geq \operatorname{val}\left(v_{\sigma}^{\prime}\right)$. Finally, $\operatorname{val}\left(v_{\sigma}\right) \geq \operatorname{val}\left(v_{\sigma}^{\prime}\right)$. By the minimality of $v_{\sigma}$ it follows that $v_{\sigma}=v_{\sigma}^{\prime}$ and $\operatorname{val}\left(v_{\sigma}\right)=\psi^{-1 *}\left(\operatorname{val}\left(v_{\tau}\right)\right)=\operatorname{val}\left(v_{\sigma}^{\prime}\right)$, or equivalently $\psi^{*}\left(\operatorname{val}\left(v_{\sigma}\right)\right)=\operatorname{val}\left(v_{\tau}\right)$.

We need to show that $v_{\tau}$ is a minimal internal vector. Suppose it isn't. Write $v_{\tau}=v_{\tau 0}+v_{\tau 1}$, where $v_{\tau 0} \in \operatorname{int}(\tau)$ and $v_{\tau 1} \in \tau$. By the above find $v_{\sigma 0} \in \operatorname{int}(\sigma)$ such that $\operatorname{val}\left(v_{\sigma 0}\right)=\psi^{-1 *}\left(\operatorname{val}\left(v_{\tau 0}\right)\right)$. Then $\operatorname{val}\left(v_{\sigma}\right)=\psi^{-1 *}\left(\operatorname{val}\left(v_{\tau}\right)\right) \geq \psi^{-1 *}\left(\operatorname{val}\left(v_{\tau 0}\right)\right)=\operatorname{val}\left(v_{\sigma 0}\right)$. By the minimality of $v_{\sigma}$, we get $v_{\sigma}=v_{\sigma 0}$ and $v_{\tau}=v_{\tau 0}$.

Lemma 5.3.9. Let $\sigma$ be a $\Gamma$-semicone and $v \in \sigma$ be a vector such that for any $\phi \in G^{\sigma}$ there exists $v_{\phi} \in \sigma$ such that $\phi^{*}(\operatorname{val}(v))=\operatorname{val}\left(v_{\phi}\right)$. Then $\operatorname{val}(v)$ is $G^{\sigma}$-invariant.

Proof. Set $X:=\widetilde{X}_{\sigma}, S:=S_{\widetilde{\sigma}}, x:=O_{\tilde{\sigma}}$. Let $W$ denote the set of all vectors $v_{\phi} \in \sigma$ for all $\phi \in \operatorname{Aut}(X, S)$. For any natural $a$, the ideals $I_{\mathrm{val}\left(v_{\phi}\right), a}:=\left\{f \in \widehat{\mathcal{O}}_{X, x} \mid \operatorname{val}\left(v_{\phi}\right)(f) \geq a\right\}$ are generated by monomials. They define the same Hilbert-Samuel function $k \mapsto \operatorname{dim}_{K}\left(K[X] /\left(I+m^{k}\right)\right)$, where $m$ denotes the maximal ideal. It follows that the set $W$ is finite.

On the other hand since the ideals $I_{\mathrm{val}\left(v_{\phi}\right), a}$ are generated by monomials they can be distinguished by the ideals $\operatorname{gr}\left(I_{\mathrm{val}\left(v_{\phi}\right), a}\right)$ in the graded ring

$$
\operatorname{gr}\left(\widehat{\mathcal{O}}_{X, x_{\sigma}}\right)=\widehat{\mathcal{O}}_{X, x} / m_{x, X} \oplus m_{x, X_{\sigma}} / m_{x, X_{\sigma}}^{2} \oplus \ldots,
$$

where $m_{x, X} \subset \widehat{\mathcal{O}}_{X, x}$ is the maximal ideal of the point $x$.

Let $d: \operatorname{Aut}\left(\widetilde{X}_{\sigma}, S_{\sigma}\right)^{0} \rightarrow \operatorname{Gl}\left(\operatorname{Tan}_{X, x}\right)$ be the differential morphism (see Example 4.5.4). Then the connected algebraic group $d\left(\operatorname{Aut}\left(\widetilde{X}_{\sigma}, S_{\sigma}\right)^{0}\right)$ acts algebraically on the connected component of the Hilbert scheme of graded ideals with fixed Hilbert polynomial (see Example 4.5.4) . In particular it acts trivially on the finite subset $\operatorname{gr}\left(I_{\mathrm{val}\left(v_{\phi}\right), a}\right)$, and consequently $\operatorname{Aut}\left(\widetilde{X}_{\sigma}, S_{\sigma}\right)^{0}$ preserves all $I_{\mathrm{val}\left(v_{\phi}\right), a}$.

Lemma 5.3.10. Let $\sigma$ be a $\Gamma$-semicone and $\widetilde{X}_{\Delta^{\sigma}} \rightarrow \widetilde{X}_{\sigma}$ be a $G^{\sigma}$-equivariant morphism. Then all its exceptional divisors are $G^{\sigma}$-invariant.

Proof. Any automorphism $g \in G^{\sigma}$ maps an exceptional divisor $D$ to another exceptional divisor $D^{\prime}$. Hence the valuation $\mathrm{val}_{D}$ defined by $D$ satisfies the condition of the previous lemma, and therefore it is $G^{\sigma}$-invariant.

Definition 5.3.11. Let $\sigma$ be a $\Gamma$-semicone. A valuation $\operatorname{val}(v)$, where $v \in \sigma$, is $G^{\sigma}$-semiinvariant if for any $\phi \in G^{\sigma}$ there exist $v^{\prime} \in \sigma$ such that $\phi^{*}(\operatorname{val}(v))=\operatorname{val}\left(v^{\prime}\right)$.

By abuse of terminology a vector $v \in \sigma$ will be called $G^{\sigma}$-semiinvariant (resp. $G^{\sigma}$-invariant) if the corresponding valuation $\operatorname{val}(v)$ is $G^{\sigma}$-semiinvariant (resp. $G^{\sigma}$-invariant).

Definition 5.3.12. Let $\Sigma$ be an oriented $\Gamma$-semicomplex. $v \in \operatorname{int}(\sigma)$ is semistable if for any $\tau \geq \sigma, \operatorname{val}(v)$ is $G^{\tau}$-semiinvariant on $\widetilde{X}_{\tau}$.

Let $\operatorname{Vert}(\Sigma)$ denote the set of all 1-dimensional rays in the fan $\Sigma$.

Definition 5.3.13. Let $\sigma$ be a $\Gamma$-semicone and $\widetilde{X}_{\Delta^{\sigma}} \rightarrow \widetilde{X}_{\sigma}$ be a $G^{\sigma}$-equivariant morphism. A cone $\delta \in \Delta^{\sigma}$ is $G^{\sigma}$-invariant if

1. there is $\tau \leq \sigma$ such that $\operatorname{int}(\delta) \subset \operatorname{int}(\tau)$,

2. $\bar{O}_{\delta} \subset \widetilde{X}_{\Delta^{\sigma}}$ is $G^{\sigma}$-invariant.

Definition 5.3.14. Let $\Delta$ be a canonical subdivision of an oriented $\Gamma$-semicomplex $\Sigma$. A cone $\delta \in \Delta^{\sigma}$, $\sigma \in \Sigma$ is called a $\Sigma$-stable face of $\Delta$ if

1. $\operatorname{int}(\delta) \subset \operatorname{int}(\sigma)$,

2. $\imath_{\sigma}^{\tau}(\delta) \in \Delta^{\tau}$ is $G^{\tau}$-invariant for any $\tau \geq \sigma$. 
Lemma 5.3.15. Let Let $\sigma$ be a $\Gamma$-semicone and $\widetilde{X}_{\Delta^{\sigma}} \rightarrow \widetilde{X}_{\sigma}$ be a $G^{\sigma}$-equivariant morphism.

1. All $G^{\sigma}$-semiinvariant vectors $v \in \sigma$ are $G^{\sigma}$-invariant.

2. $\operatorname{Vert}\left(\Delta^{\sigma}\right) \backslash \operatorname{Vert}(\bar{\sigma})$ are $G^{\sigma}$-invariant.

3. Let $\delta$ be a $G^{\sigma}$-invariant face of $\Delta^{\sigma}$. Then any minimal internal vector $v \in \operatorname{int}(\delta)$ are $G^{\sigma}$-invariant.

4. Let $\delta$ be a $\Gamma$-indecomposable face of $\Delta^{\sigma}$. Then $\delta$ is $G^{\sigma}$-invariant and all its minimal internal vectors $v$ are $G^{\sigma}$-invariant. In particular all minimal generators of any face $\delta \in \Delta^{\sigma}$ are $G^{\sigma}$-invariant.

5. Let $\delta \in \Delta^{\sigma}$ be a minimal face such that $\operatorname{int}(\delta) \subset \operatorname{int}(\tau)$, where $\tau \neq\{0\}$. Then $\delta$ is $G^{\sigma}$-invariant and $\operatorname{int}(\delta)$ contains a $G^{\sigma}$-invariant vector.

Proof. (1) Follows from Lemma 5.3.9. (2) Follows from Lemma 5.3.10. (3) By Lemma 5.3.8, $v$ is $G^{\sigma}-$ semiinvariant. By $(1), v$ is $G^{\sigma}$-invariant.

(4) Let $\tau \preceq \sigma$ be a face of $\sigma$ such that $\operatorname{int}(\delta) \subset \operatorname{int}(\tau)$. By Lemmas 4.12.8 and 4.12.5, $\Delta^{\sigma}\left|\tau=\Delta^{\sigma}\right| \omega(\tau) \oplus r(\tau)$ for some regular cone $r(\tau)$. Since $\delta$ is $\Gamma$-indecomposable we conclude that $\delta \in \Delta^{\sigma} \mid \omega(\tau)$ and $\tau=\omega(\tau) \leq \sigma$. By definition $G^{\sigma}$ acts on $\widetilde{X}_{\Delta^{\sigma}}$ and consequently on strata from the stratification $\operatorname{Sing}^{\Gamma}\left(\widetilde{X}_{\Delta^{\sigma}}\right)$ (see Lemma 4.7.4.

Let $\operatorname{sing}_{0} \in \operatorname{Sing}\left(\widetilde{X}_{\Delta^{\sigma}}\right)$ denote the stratum corresponding to indecomposable face $\delta_{0} \in \Delta^{\sigma}$.

The images of the $G^{\sigma}$ action on $\operatorname{sing}_{0}$ form a finite invariant subset of strata $\operatorname{Sing}_{0} \subset \operatorname{Sing}\left(\widetilde{X}_{\Delta^{\sigma}}\right)$. All strata from $\operatorname{Sing}_{0}$ correspond to some isomorphic cones $\delta_{i} \in \Delta^{\sigma}$ for $i=0, \ldots, l$. Let $v \in \operatorname{int}(\delta)$ be a minimal internal vector. Then by Lemma 5.3 .8 for any $\widetilde{\phi} \in G^{\sigma}$ the image $\widetilde{\phi}_{*}(\operatorname{val}(v))$ is a valuation on $\widetilde{X}_{\Delta^{\tau}}$ corresponding to a minimal internal vector of $\delta_{i}$. Hence $v$ is $G^{\sigma}$-semiinvariant and by (1) it is $G^{\sigma}$-invariant. By Lemma 2.1.3 each minimal generator is a minimal internal vector of some indecomposable cone $\delta$, hence by the above it is $G^{\sigma}$-invariant.

(5) For any $\tau \geq \sigma$ the cone $\delta$ corresponds to the maximal component $O_{\delta}$ in $\tilde{X}_{\Delta^{\sigma}}$ which dominates $O_{\tau} \subset \tilde{X}_{\sigma}$. Then $G^{\sigma}$ permutes the set of maximal components of the inverse image of $O_{\tau}$ dominating $O_{\tau}$ and we repeat the reasoning from (4).

Remark. The above lemma holds if we replace $G^{\sigma}$ with its connected proalgebraic subgroup.

As a direct corollary of Lemma 5.3.15 is the following

Lemma 5.3.16. Let $\Delta$ be a canonical subdivision of an oriented $\Gamma$-semicomplex $\Sigma$.

1. All the semistable vectors in $\Sigma$ are stable.

2. For any $\sigma \in \Sigma$ all elements of $\operatorname{Vert}\left(\Delta^{\sigma}\right) \backslash \operatorname{Vert}(\bar{\sigma})$ are $\Sigma$-stable.

3. Let $\delta$ be a $\Sigma$-stable face of $\Delta$. Then any minimal internal vector $v \in \operatorname{int}(\delta)$ is $\Sigma$-stable.

4. Let $\delta$ be a $\Gamma$-indecomposable face of $\Delta^{\sigma}$. Then $\delta$ is $\Sigma$-stable and all its minimal internal vectors are stable. In particular all minimal generators of any face $\delta \in \Delta^{\sigma}$ are $\Sigma$-stable.

5. Let $\delta \in \Delta^{\sigma}$ be a minimal face such that $\operatorname{int}(\delta) \subset \operatorname{int}(\sigma)$, where $\sigma \neq\{0\}$. Then $\delta$ is $\Sigma$-stable and int $(\delta)$ contains a $\Sigma$-stable vector.

\subsection{Star subdivisions and blow-ups of stable valuations.}

Proposition 5.4.1. $\quad$ 1. Let $\Sigma$ be an oriented $\Gamma$-semicomplex and $v_{1}, \ldots, v_{k}$ be $\Sigma$-stable vectors. Then $\Delta:=\left\langle v_{k}\right\rangle \cdot \ldots \cdot\left\langle v_{1}\right\rangle \cdot \Sigma$ is a canonical subdivision of $\Sigma$.

2. Let $(X, S)$ be a $\Gamma$-stratified toroidal variety with an associated oriented $\Gamma$-semicomplex $\Sigma$. Let $v_{1}, \ldots, v_{k}$ be stable vectors and $\nu_{1}, \ldots, \nu_{k}$ be the associated stable valuations. Then the composite of blow-ups $\mathrm{bl}_{\nu_{k}} \circ \ldots \circ \mathrm{bl}_{\nu_{1}}: X_{k} \rightarrow X$ is the toroidal modification of $X$ associated to the canonical subdivision $\Delta:=\left\langle v_{k}\right\rangle \cdot \ldots \cdot\left\langle v_{1}\right\rangle \cdot \Sigma$ of $\Sigma$.

Proof. Induction on $k$. Let $\Delta_{k}:=\left\langle v_{k}\right\rangle \cdot \ldots \cdot\left\langle v_{1}\right\rangle \cdot \Sigma$ be a canonical subdivision corresponding to $f:=$ $\mathrm{bl}_{\nu_{k}} \circ \ldots \circ \mathrm{bl}_{\nu_{1}}: X_{k} \rightarrow X$. Then $v_{k+1} \in \operatorname{int}(\sigma)$ defines an $X$-stable valuation $\nu_{k+1}$ on $X_{k}$. It follows from 5.3 .7 that for any chart $\phi_{\sigma}: U_{\sigma} \rightarrow X_{\sigma}$ the blow-up of $f^{-1}\left(U_{\sigma}\right) \subset X_{j}$ at $\nu_{k+1}=\phi^{f *}\left(\operatorname{val}\left(v_{k+1}\right)\right)$ corresponds to the star subdivision of $\Delta_{k}^{\sigma}$ at $v_{k+1}$. 


\section{CORRESPONDENCE BETWEEN TOROIDAL MORPHISMS AND CANONICAL SUBDIVISIONS}

6.1. Definition of stable support. By Lemma 5.3.3 we are in a position to glue pieces stab $(\sigma)$ into one topological space.

Definition 6.1.1. The stable support of a $\Gamma$-semicomplex $\Sigma$ is the topological space $\operatorname{Stab}(\Sigma):=\bigcup_{\sigma \in \Sigma} \operatorname{stab}(\sigma)$.

Let $G \subset \operatorname{Aut}\left(\widetilde{X}_{\sigma}\right)$ be any abstract algebraic group. Let $I_{G}$ denote the set of $G$-invariant toric valuations on $\widetilde{X}_{\sigma}$. Set

$$
\operatorname{Inv}(G, \sigma):=\left\{a \cdot v \mid \operatorname{val}(v) \in I_{G}, a \in \mathbf{Q}_{\geq 0}\right\}, \quad \operatorname{Inv}(\sigma):=\operatorname{Inv}\left(G^{\sigma}, \sigma\right) .
$$

Lemma 6.1.2. Let $\Sigma$ be a $\Gamma$-semicomplex.

1. $\operatorname{stab}(\sigma) \cap \operatorname{int}(\sigma)=\bigcap_{\tau \geq \sigma} \operatorname{Inv}(\tau) \cap \operatorname{int}(\sigma)$.

2. $\operatorname{stab}(\sigma)=\bigcup_{\tau \leq \sigma}(\operatorname{stab}(\tau) \cap \operatorname{int}(\tau))$.

Proof. Follows from the definitions of stable valuation and stable support.

\subsection{Convexity of the stable support.}

Lemma 6.2.1. 1. For any abstract subgroup $G \subset \operatorname{Aut}\left(\widehat{X}_{\sigma}\right)$ the cone $\operatorname{Inv}(G, \sigma)$ is convex.

2. Let $\Sigma$ be a $\Gamma$-semicomplex. Then for any $\sigma \in \Sigma$, $\operatorname{stab}(\sigma)$ is convex.

Proof. (1). Let $v_{1}, v_{2} \in I_{G}$ and $\mathrm{bl}_{\mathrm{val}\left(v_{1}\right)} \circ \mathrm{bl}_{\mathrm{val}\left(v_{2}\right)}: X_{\left\langle v_{1}\right\rangle \cdot\left\langle v_{2}\right\rangle \cdot \sigma} \rightarrow X_{\sigma}$ be the toric morphism. The induced morphism $\left(\mathrm{bl}_{\mathrm{val}\left(v_{1}\right)} \circ \mathrm{bl}_{\mathrm{val}\left(v_{2}\right)}\right)^{\top}: X_{\left\langle v_{1}\right\rangle \cdot\left\langle v_{2}\right\rangle \cdot \sigma} \times_{X_{\sigma}} \widehat{X}_{\sigma} \rightarrow \widehat{X}_{\sigma}$ is $G$-equivariant. The exceptional divisors $D_{1}, D_{2}$ of $\mathrm{bl}_{\mathrm{val}\left(v_{1}\right)} \circ \mathrm{bl}_{\mathrm{val}\left(v_{2}\right)}$ correspond to $v_{1}, v_{2} \in \sigma$. The $D_{1}$ and $D_{2}$ intersect along a stratum $O_{\tau}$ corresponding to the cone $\tau:=\left\langle v_{1}, v_{2}\right\rangle$. Then $\widehat{X}_{\tau}$ is a $G$-invariant local scheme of toric variety at the generic point of the orbit $O_{\tau}$ and $\left\{D_{1}, D_{2}, O_{\tau}\right\}$ is the orbit stratification on $\left(\widehat{X}_{\tau}\right)$ which is also $G$-invariant.

Let $u_{1}, \ldots, u_{k}$ denote semiinvariant generators of $\widehat{\mathcal{O}}_{X_{\tau}, O_{\tau}} \simeq K\left(O_{\tau}\right)\left[\left[u_{1}, \ldots, u_{k}\right]\right]$ Each automorphism from $G$ preserves the orbit stratification and multiplies the generating monomials $u_{i}$ by invertible functions. Therefore it does not change the valuations $\operatorname{val}(v)$, where $v \in \tau$, on $\widehat{\mathcal{O}}_{X_{\tau}, O_{\tau}} \simeq K\left(O_{\tau}\right)\left[\left[u_{1}, \ldots, u_{k}\right]\right]$.

(2) Follows from (1) and from Lemma 6.1.2

\subsection{Toroidal embeddings and stable support.}

Lemma 6.3.1. Let $(X, S)$ be a toroidal embedding. Then

1. All integral vectors in the faces $\sigma$ of $\Sigma$ are stable.

2. All subdivisions of $\Sigma$ are canonical.

Proof. (1) Each automorphism $g$ from $G^{\sigma}$ preserves divisors, hence multiplies the generating monomials by invertible functions. Consequently, it does not change the valuations val $(v)$, where $v \in \sigma$, on $\widetilde{X}_{\sigma}$.

(2) Let $\Delta^{\sigma}$ be a subdivision of $\sigma$. For any $\delta \in \Delta^{\sigma}$ each automorphism $g$ of $\tilde{X}_{\sigma}$ lifts to an automorphism $g^{\prime}$ of $\widetilde{X}_{\delta}=\widetilde{X}_{\sigma} \times_{X_{\tilde{\sigma}}} X_{\widetilde{\delta}}$ which also multiplies monomials by suitable invertible functions. Therefore $g$ lifts to the scheme $\tilde{X}_{\Delta^{\sigma}}$.

\subsection{Minimal vectors and stable support.}

Lemma 6.4.1. Let $\sigma$ be a $\Gamma$-semicone and $\tilde{X}_{\Delta^{\sigma}} \rightarrow \tilde{X}_{\sigma}$ be a $G^{\sigma}$-equivariant morphism.

1. Let $\delta \in \Delta^{\sigma}$ be a simplicial cone where $\sigma \in \Sigma$. Then all vectors in $\operatorname{par}(\delta)$ are $\Sigma$-stable.

2. Let $\delta \in \Delta^{\sigma}$ be a $G^{\sigma}$-invariant simplicial face. Then all vectors from $\overline{\operatorname{par}(\delta)} \cap \operatorname{int}(\delta)$ are $G^{\sigma}$-invariant.

Proof. (1) Each $v \in \operatorname{par}(\delta)$ is a nonnegative integral combination of minimal generators of $\delta$. Minimal generators of $\delta$ are $G^{\sigma}$-invariant by Lemma 5.3.15(4). Their linear combination is $G^{\sigma}$-invariant by Lemma 6.2 .1 .

(2) Let $v \in \overline{\operatorname{par}(\delta)} \cap \operatorname{int}(\delta)$. Write $\delta=\left\langle w_{1}, \ldots, w_{k}\right\rangle$ and $v=\sum \alpha_{i} w_{i}$, where $0 \leq \alpha_{i} \leq 1$. If $v$ is a minimal internal vector then it is $G^{\sigma}$-invariant by Lemma 5.3.15(3). If not then $v=v^{\prime}+v^{\prime \prime}$, where $v^{\prime}=\sum \beta_{i} w_{i}$, 
$0<\beta_{i} \leq \alpha_{i} \leq 1$, is a minimal internal vector and $v^{\prime \prime}=\sum\left(\alpha_{i}-\beta_{i}\right) w_{i} \in \operatorname{par}(\delta)$. By (1) and Lemma 6.2.1, $v^{\prime}$ is $G^{\sigma}$-invariant.

A direct corollary from the abo Lemma is the following Lemma.

Lemma 6.4.2. Let $\Delta$ be a canonical subdivision of an oriented $\Gamma$-semicomplex $\Sigma$.

1. Let $\delta \in \Delta^{\sigma}$ be a simplicial cone where $\sigma \in \Sigma$. Then all vectors in $\operatorname{par}(\delta)$ are $\Sigma$-stable.

2. Let $\delta \in \Delta^{\sigma}$ be a $\Sigma$-stable simplicial cone. Then all vectors from $\overline{\operatorname{par}(\delta)} \cap \operatorname{int}(\delta)$ are $\Sigma$-stable.

\subsection{Canonical stratification on a toroidal modification.}

Lemma 6.5.1. Let $\Delta$ be a canonical subdivision of an oriented $\Gamma$-semicomplex $\Sigma$. For any $\sigma \in \Sigma$ set

$$
\Delta_{\text {stab }}^{\sigma}:=\left\{\delta \in \Delta^{\sigma} \mid \operatorname{int}(\delta) \cap \operatorname{stab}(\sigma) \neq \emptyset\right\} .
$$

Then

1. $\Delta_{\text {stab }}^{\sigma} \subset \Delta^{\sigma}$ is an embedded $\Gamma_{\sigma}$-semifan in $N_{\sigma}$.

2. The subset $\Delta_{\text {stab }}^{\sigma} \subset \Delta^{\sigma}$ consists of all $\Sigma$-stable faces in $\Delta^{\sigma}$.

3. For any $\tau \leq \sigma, \Delta_{\text {stab }}^{\sigma} \mid \imath_{\tau}^{\sigma}(\tau)=\imath_{\tau}^{\sigma}\left(\Delta_{\text {stab }}^{\tau}\right)$.

4. For any $\sigma \in \Sigma$, the stratification $S_{\sigma}^{\text {stab }}$ on $\widetilde{X}_{\Delta^{\sigma}}$ determined by the embedded $\Gamma$-semifan $\Delta_{\text {stab }}^{\sigma} \subset \Delta^{\sigma}$ is $G^{\sigma}$-invariant.

Proof. (1) By Lemma 6.2.1, stab $\sigma)$ is convex and there is a unique maximal face $\omega \preceq \delta$ whose relative interior $\operatorname{int}(\omega)$ intersects the stable support. Thus $\omega \in \Delta_{\text {stab }}^{\sigma}$. By Lemma 5.3.16 $(4)$, either $\operatorname{sing}^{\Gamma}(\delta)=\{0\}$ or $\operatorname{int}\left(\operatorname{sing}^{\Gamma}(\delta)\right) \cap \operatorname{stab}(\sigma) \neq \emptyset$. In both cases $\operatorname{sing}^{\Gamma}(\delta) \leq \omega$ and consequently $\delta=\omega \oplus^{\Gamma} r(\delta)$, for some regular cone $r(\delta)$.

(2) If $\delta \in \Delta^{\sigma}$ is $\Sigma$-stable then by Lemma 5.3.16(3) its minimal internal vector is $\Sigma$-stable. Therefore $\delta \in \Delta_{\text {stab }}^{\sigma}$. If the relative interior of $\delta \in \Delta_{\text {stab }}^{\sigma}$ contais a stable vector $v \in \operatorname{int}(\tau)$, where $\tau \leq \sigma$, then $\operatorname{int}(\delta) \subset \operatorname{int}(\tau), \delta \in \Delta_{\text {stab }}^{\tau}$, and for any $\varrho \geq \tau$, the closure of $O_{\imath} \varrho(\delta) \subset \widetilde{X}_{\Delta} \varrho$ is exactly the center of val $(v)$, and therefore is $G^{\varrho}$-invariant.

(3) (4) follow from (2).

As a simple corollary of the above we get

Lemma 6.5.2. There is a $\Gamma$-semicomplex $\Delta_{\text {stab }}$ obtained by glueing the semicomplexes $\Delta_{\text {stab }}^{\sigma \text { semic }}$ along $\Delta_{\text {stab }}^{\tau \text { semic }}$, where $\tau \leq \sigma$.

Proof For any $\omega \in \Delta_{\text {stab }}^{\sigma \text { semic }}$ denote by $\sigma(\omega \leq \sigma$ the semicone in $\Sigma$ for which $\operatorname{int}(\omega) \subset \operatorname{int}(\sigma(\omega))$. Then

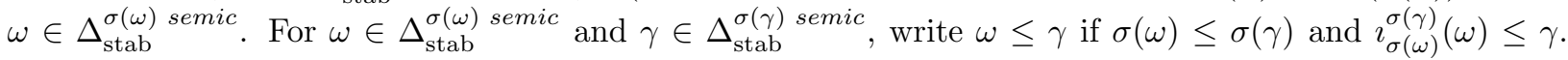
Then for $\omega \leq \gamma$ we set $\imath_{\omega}^{\gamma}:=\imath_{\sigma(\omega)}^{\sigma(\gamma)}$ and $\Gamma_{\omega}:=\left(\Gamma_{\sigma(\omega)}\right)_{\omega}$.

Proposition 6.5.3. Let $(X, S)$ be an oriented $\Gamma$-stratified toroidal variety with an associated oriented $\Gamma$ semicomplex $\Sigma$. Let $Y$ be a toroidal modification of $(X, S)$ corresponding to a canonical subdivision $\Delta$ of $\Sigma$. Then there is a canonical stratification $R$ on $Y$ with the following properties:

1. Let $\phi_{\sigma}^{f}: f^{-1}\left(U_{\sigma}\right) \rightarrow X_{\Delta^{\sigma}}$ denote the morphism induced by a chart $\phi_{\sigma}: U_{\sigma} \rightarrow X_{\sigma}$. The intersections $r \cap f^{-1}\left(U_{\sigma}\right), r \in R$ are precisely the inverse images of the strata associated to the embedded $\Gamma$-semifan $\Delta_{\text {stab }}^{\sigma} \subset \Delta^{\sigma}$.

2. The closures $\bar{r}$ of strata $r \in R$ are centers of $X$-stable valuations on $Y$.

3. The $\Gamma$-semicomplex associated to $(Y, R)$ is equal to $\Sigma_{R}=\Delta_{\text {stab }}$ and the atlas is given by $\bigcup_{\sigma \in \Sigma} \mathcal{U}\left(\Delta^{\sigma}, \Delta_{\text {stab }}^{\sigma}\right)$.

4. The stratification $R$ is the finest stratification on $Y$ satisfying the conditions:

(a) The morphism $f$ maps strata in $R$ onto strata in $S$.

(b) There is an embedded semifan $\Omega^{\sigma} \subset \Delta^{\sigma}$ such that the intersections $r \cap f^{-1}\left(U_{\sigma}\right), r \in R$, are inverse images of strata of $\left.S_{\Omega^{\sigma}}\right)$ on $\left(X_{\Delta^{\sigma}}\right.$.

(c) For any point $x$ every $\Gamma_{x}$-equivariant automorphism $\alpha$ of $\widehat{X}_{x}$ preserving strata and orientation can be lifted to an automorphism $\alpha^{\prime}$ of $Y \times_{X} \widehat{X}_{x} \rightarrow Y$ preserving strata.

Proof. For any face $\omega \in \Delta_{\text {stab }}^{\sigma}$ we find a $\Sigma$-stable vector $v_{\omega}$ in its relative interior. By Lemma 5.3 .6 the vector $v_{\omega}$ corresponds to an $X$-stable valuation $\nu_{\omega}$ on $Y$. We define the closure of a stratum $r \in R$ asociated 
to $\omega$ to be

$$
\bar{r}:=\overline{\operatorname{strat}_{Y}(\omega)}:=\mathrm{Z}\left(\nu_{\omega}\right)
$$

Then define the stratum $r$ as

$$
r:=\operatorname{strat}_{Y}(\omega):=\overline{\operatorname{strat}_{Y}(\omega)} \backslash \bigcup_{\omega^{\prime}<\omega} \overline{\operatorname{strat}_{Y}\left(\omega^{\prime}\right)}
$$

By Lemma 3.1 .8 the strata $\operatorname{strat}(\omega)$ of the stratification associated to the embedded semifan $\Delta_{\text {stab }}^{\sigma} \subset \Delta^{\sigma}$ satisfy the condition $\overline{\operatorname{strat}(\omega)}=\mathrm{Z}\left(\operatorname{val}\left(v_{\omega}\right), X_{\Delta^{\sigma}}\right), \operatorname{strat}(\omega):=\overline{\operatorname{strat}(\omega)} \backslash \bigcup_{\omega^{\prime}<\omega} \overline{\operatorname{strat}\left(\omega^{\prime}\right)}$. It follows by the above that the sets $r$ define stratification $R$ satisfying conditions (1), (2) and (3).

(4) Since the strata of $R$ on $Y$ are defined by centers of $X$-stable valuations, conditions (a), (b) and (c) are satisfied.

Let $Q$ be a stratification on $Y$ satisfying conditions (4a), (4b) and (4c).

For the morphism $\phi_{\sigma}^{f}$ induced by a chart $\phi_{\sigma}$ and for any $x \in U_{\sigma}$, let $\widetilde{\phi}_{\sigma}^{f}: Y \times_{X} \widehat{X}_{x} \rightarrow \widetilde{X}_{\Delta^{\sigma}}$ be a $G^{\sigma} \simeq \operatorname{Aut}\left(\widehat{X}_{x}, S\right)^{0}$-equivariant isomorphism of formal completions mapping the strata of $Q$ isomorphically onto the strata of $\widetilde{S}_{\Omega^{\sigma}}$ on $\widetilde{X}_{\Delta^{\sigma}}$ defined by the embedded semifan $\Omega^{\sigma} \subset \widetilde{\Delta}^{\sigma}$

By (4a) the strata of $\widetilde{S}_{\Omega^{\sigma}}$ on $\widetilde{X}_{\Delta^{\sigma}}$ are $G^{\sigma}$-invariant. Hence for any two isomorphisms $\widetilde{\phi}_{\sigma, i}^{f}: Y \times_{X} \widehat{X}_{x} \rightarrow$ $\widetilde{X}_{\Delta^{\sigma}}$, where $i=1,2$, induced by charts $\phi_{\sigma, i}$ the induced automorphism $\widetilde{\phi}_{\sigma, 1}^{f}\left(\widetilde{\phi}_{\sigma, 2}^{f}\right)^{-1} \in G^{\sigma}$ of $\widetilde{X}_{\Delta^{\sigma}}$ maps $\widetilde{S}_{\Omega_{1}^{\sigma}}$ to $\widetilde{S}_{\Omega_{2}^{\sigma}}$. Since both stratifications are $G^{\sigma}$-invariant we get $\widetilde{S}_{\Omega_{1}^{\sigma}}=\widetilde{S}_{\Omega_{2}^{\sigma}}, \Omega_{1}^{\sigma}=\Omega_{2}^{\sigma}$. Hence $\Omega^{\sigma}$ does not depend upon a chart. Consequently, if $\tau \leq \sigma$ then $\Omega^{\sigma} \mid \tau=\imath_{\tau}^{\sigma}\left(\Omega^{\tau}\right)$. By condition (4a), the relative interior of a face $\omega \in \Omega^{\sigma}$ is contained in the interior of a face $\tau \leq \sigma$. Moreover the closure $O_{\imath_{\tau}(\omega)} \subset \tilde{X}_{\Omega \varrho}$ is $G^{\varrho}$-invariant for any $\varrho \geq \tau$. This shows that all faces $\omega \in \Omega^{\sigma}$ are $\Sigma$-stable. Consequently, $\Omega^{\sigma} \subset \Delta_{\text {stab }}^{\sigma}$ and finally by Lemma 3.1.9. the corresponding stratification $S_{\Delta^{\sigma}}$ is finer than $S_{\Omega^{\sigma}}$.

6.6. Correspondence between toroidal morphisms and canonical subdivisions. Proposition 6.5.3 can be rephrased as follows:

Theorem 6.6.1. Let $(X, S)$ be an oriented stratified toroidal variety (resp. $\Gamma$-stratified toroidal variety) with the associated oriented semicomplex (resp. $\Gamma$-semicomplex) $\Sigma$. There is a $1-1$ correspondence between the toroidal morphisms of stratified (resp. $\Gamma$-stratified) toroidal varieties $f:(Y, R) \rightarrow(X, S)$ and canonical subdivisions $\Delta$ of the oriented semicomplex (resp. orineted $\Gamma$-semicomplex) $\Sigma$. Moreover the semicomplex (resp. $\Gamma$-semicomplex) associated to $(Y, R)$ is given by $\Sigma_{R}=\Delta_{\text {stab }}$.

Remark. In particular, if $(X, S)$ is a toroidal embedding (see Lemma 6.3.1) then $\Sigma$ is a complex and all its subdivisions are canonical (Lemma 6.3.1). We get a 1-1-correspondence between the subdivisions of the complex $\Sigma$ and the toroidal morphisms $(Y, R) \rightarrow(X, S)$ (see [39]).

Example 6.6.2. Let $X$ be a variety with isolated singularity of type $x_{1} x_{2}=x_{3} x_{4}$ with the stratification consisting of the singular point and its complement (as in Example 4.10.7). Then the associated semicomplex $\Sigma$ consists of the cone over a square and its vertex. As follows from Example 13.3.1 the stable support consists of the ray over the center of this square. Consequently, by Proposition 7.6.1 there are three canonical nontrivial subdivisions of $\Sigma$. One is the star subdivision at the stable ray corresponding to the blow-up of the point. Then $\Delta_{\text {stab }}$ consists of the ray and its vertex and corresponds to the toroidal embedding defined by the exceptional divisor and its complement. The other two are subdivisions defined by diagonals corresponding to two small resolutions of singularities. $\Delta_{\text {stab }}$ consists of the cone over the diagonal and its vertex and corresponds to the smooth stratified toroidal variety with the stratification defined by the exceptional curve-the preimage of the singular point and its complement.

The language of stratified toroidal varieties allows a combinatorial description of the Hironaka twist.

Example 6.6.3. (29) Let $X=\mathbf{P}^{3}$ be a projective 3 -space containing two curves $l_{1}$ and $l_{2}$ intersecting transversally in two points $p_{1}$ and $p_{2}$. These data define a stratified toroidal variety $(X, S)$. The associated semicomplex $\Sigma$ consists of two 3 -dimensional cones $\sigma_{1}$ and $\sigma_{2}$ corresponding respectively to the points $p_{1}$ and $p_{2}$, and sharing two 2-dimensional faces $\tau_{1}$ and $\tau_{2}$ corresponding to $l_{1}$ and $l_{2}$. The Hironaka twist $Y$ is obtained by glueing the consecutive blow-ups of $X \backslash\left\{p_{i}\right\}$ at $l_{1}$ and $l_{2}$ taken in two different orders, along the isomorphic open subsets over $X \backslash\left\{p_{1}\right\} \backslash\left\{p_{2}\right\}$. Then $Y$ is a stratified toroidal variety. The preimage of $p_{i}$ 
JAROSŁAW WŁODARCZYK

consists of two irreducible curves $l_{i 1}$ and $l_{i 2}$ intersecting at $p_{i 0}$. The stratification $T$ on $Y$ is determined by the above four curves, two points and two exceptional divisors-preimages of curves. Let $v_{i}$ denote the sum of the two generators of $\tau_{i}$. Let $v^{i}$ denote the sum of the generators of $\sigma_{i}$.

$\Delta$ is the subdivision of $\Sigma$ obtained by glueing the consecutive star subdivisions of $\sigma_{i}$ at $\left\langle v_{1}\right\rangle$ and $\left\langle v_{2}\right\rangle$ taken in two different orders, along the star subdivisions of $\tau_{i}$ at $\left\langle v_{i}\right\rangle$. It follows from Example 13.3.2 that $\operatorname{Stab}(\Sigma)$ is the union of the cones $\left\langle v_{1}, v_{2}, v^{1}\right\rangle \subset \sigma_{1}$ and $\left\langle v_{1}, v_{2}, v^{2}\right\rangle \subset \sigma_{2}$. Then $\Delta_{\text {stab }}$ consists of the relevant cones in $\Delta$ whose relative interior intersects $\operatorname{Stab}(\Sigma)$. These cones correspond to the above mentioned strata on $Y$.

\section{Canonical SUbdivisions and Stable SUPPORT}

\subsection{Isomorphisms of local rings and linear transformations of stable supports.}

Lemma 7.1.1. 1. Let $\sigma$ and $\tau$ be two cones of the same dimension. Let $\psi: \widehat{X}_{\sigma} \simeq \widehat{X}_{\tau}$ be an isomorphism preserving the closures of the toric orbits. Then there is a linear isomorphism $L_{\psi}: \sigma \rightarrow \tau$ such that $\psi_{*}(\operatorname{val}(v))=\operatorname{val}\left(L_{\psi}(v)\right)$ for any $v \in \sigma$.

2. Let $\sigma$ and $\tau$ be two $\Gamma$-semicones of the same dimension. Let $\psi: \widetilde{X}_{\sigma} \simeq \widetilde{X}_{\tau}$ be a $\Gamma$-equivariant isomorphism preserving strata. Then there is a linear isomorphism $L_{\psi}: \operatorname{Inv}(\sigma) \rightarrow \operatorname{Inv}\left(\sigma^{\prime}\right)$ such that $\psi_{*}(\operatorname{val}(v))=\operatorname{val}\left(L_{\psi}(v)\right)$ for any $v \in \operatorname{Inv}(\sigma)$.

Proof.

(1) The isomorphism $\psi$ maps the divisors of the orbit stratification on $\tilde{X}_{\sigma}$ to the divisors of the orbit stratification on $\tilde{X}_{\tau}$. Consequently, the local semiinvariant parameters at $O_{\sigma}$ are mapped to local parameters at $O_{\tau}$ which differ from semiinvariant ones by invertible functions. This defines a linear isomorphism of cones $L: \sigma \rightarrow \tau$ mapping faces of $\sigma$ corresponding to strata to suitable faces of $\tau$ Let $\psi_{L}: \widehat{X}_{\sigma} \simeq \widehat{X}_{\tau}$ denote the induced morphism. Then $\phi:=\psi_{L}^{-1} \psi$ is an automorphism of $\widehat{X}_{\sigma}$ preserving strata. By Lemma 6.3.1 the automorphism $\phi$ preserves all valuations, hence $\psi_{*}(\operatorname{val}(v))=\psi_{L *}(\operatorname{val}(v))=\operatorname{val}(L(v))$.

(2) Let $v_{1}, v_{2} \in \operatorname{Inv}(\sigma)$. Then $\psi_{*}\left(\operatorname{val}\left(v_{1}\right)\right)$ and $\psi_{*}\left(\operatorname{val}\left(v_{2}\right)\right)$ are $G^{\tau}$-invariant, and correspond to $v_{1}^{\prime}, v_{2}^{\prime} \in$ $\operatorname{Inv}(\tau)$. Set $\Delta^{\sigma}=\left\langle v_{2}\right\rangle\left\langle v_{1}\right\rangle \cdot \sigma$ and $\Delta^{\tau}=\left\langle v_{2}^{\prime}\right\rangle\left\langle v_{1}^{\prime}\right\rangle \cdot \tau$.

The isomorphism $\psi: \widetilde{X}_{\sigma} \simeq \widetilde{X}_{\tau}$ lifts to an isomorphism $\Psi: \widetilde{X}_{\Delta^{\sigma}} \simeq \widetilde{X}_{\Delta^{\tau}}$. Let $D_{1}$ and $D_{2}$ (resp. $D_{1}^{\prime}$ and $D_{2}^{\prime}$ ) denote the exceptional divisors on $\tilde{X}_{\Delta^{\sigma}}$ (resp. $\widetilde{X}_{\Delta^{\tau}}$ ) corresponding to $v_{1}$ and $v_{2}$ (resp. $v_{1}^{\prime}$ and $v_{2}^{\prime}$ ) The generic point of intersection $p:=D_{1} \cap D_{2}$ is mapped to the point of intersection $p^{\prime}:=D_{1}^{\prime} \cap D_{2}^{\prime}$. Set $\delta_{1}:=\left\langle v_{1}, v_{2}\right\rangle \in \Delta^{\sigma}$ and $\delta_{2}:=\left\langle v_{1}^{\prime}, v_{2}^{\prime}\right\rangle \in \Delta^{\tau}$. Then $\left(\widetilde{X}_{\delta_{1}},\left\{D_{1}, D_{2}, p\right\}\right)$ is a formal completion of a toroidal embedding which is mapped to $\left(\widetilde{X}_{\delta_{2}},\left\{D_{1}^{\prime}, D_{2}^{\prime}, p^{\prime}\right\}\right)$.

By (1) $\Psi_{*}$ determines a linear transformation on $\left\langle v_{1}, v_{2}\right\rangle$ and consequently a linear transformation of $\operatorname{Inv}(\sigma)$ which is a linear isomorphism.

Definition 7.1.2. Let $\sigma$ be a $\Gamma$-semicone. A vector $v_{\sigma} \in \operatorname{int}(\widetilde{\sigma})$ is absolutely invariant if $\operatorname{val}\left(v_{\sigma}\right)$ is invariant with respect to $\operatorname{Aut}\left(\widetilde{X}_{\sigma}\right)$.

Lemma 7.1.3. Let $\sigma$ be a $\Gamma$-semicone. There exists an absolutely invariant vector $v \in \operatorname{int}(\tilde{\sigma})$.

Proof. We can assume that $\sigma=\widetilde{\sigma}$ replacing $\sigma$, if necessary, by the $\Gamma$-semicone $\widetilde{\sigma}:=\sigma \cup|\widetilde{\sigma}|$. Let $v \in \operatorname{int}(\widetilde{\sigma})$ be a minimal internal vector which by Lemma $5.3 .16(3)$ is a stable vector. By Lemma 5.3.8 for any $g \in \operatorname{Aut}\left(\widetilde{X}_{\sigma}\right)$, $g_{*}(\operatorname{val}(v))=\operatorname{val}\left(v^{\prime}\right)$, where $v^{\prime}$, is also minimal. The set $W$ of minimal vectors is contained in $\overline{\operatorname{par}}(\sigma)$ and therefore it is finite. By the above, $\operatorname{Aut}\left(\widetilde{X}_{\sigma}\right)$ acts on $W$. Let $v_{\sigma}$ denote the sum of all the minimal vectors. By Lemma 7.1.1, Aut $\left(\widetilde{X}_{\sigma}\right)$ acts on $\operatorname{Inv}(\sigma) \supset W$ and therefore it acts trivially on $v_{\sigma}$.

\subsection{Initial terms defined by a monomial valuation.}

Lemma 7.2.1. Let $F\left(x_{1}, \ldots, x_{k}\right) \in K\left[x_{1}, \ldots, x_{k}\right]$ be a quasihomogeneous polynomial of weight w $(F)$ with respect to weights $w\left(x_{1}\right), \ldots, w\left(x_{k}\right)$. Let $\nu$ be a valuation of a ring $R$ and $u_{1}, \ldots, u_{k} \in R$ be such that $\nu\left(u_{i}\right) \geq w\left(x_{i}\right)$. Then $\nu\left(F\left(u_{1}, \ldots, u_{k}\right)\right) \geq w(F)$. If $F$ belongs to the ideal $\left(x_{l}, \ldots, x_{k}\right)$ and $\nu\left(u_{i}\right)>w\left(x_{i}\right)$ for $i=l, \ldots, k$ then $\nu\left(F\left(u_{1}, \ldots, u_{k}\right)\right)>w(F)$.

Proof. The proof can be reduced to the situation, in which case $F$ is a monomial when it immediately follows from the definition of valuation. 
Definition 7.2.2. Let $\nu$ be a monomial valuation of $K\left[\left[x_{1}, \ldots, x_{k}\right]\right]$ with a basis $x_{1}, \ldots, x_{k}$ and weights $a_{1}, \ldots, a_{k}>0$. For $f \in K\left[\left[x_{1}, \ldots, x_{k}\right]\right]$ let $f=f_{0}+f_{1}+\ldots$ be a decomposition of $f$ into an infinite sum of quasihomogeneous polynomials such that $\nu\left(f_{0}\right)<\nu\left(f_{1}\right)<\ldots$. Then by the initial term of $f$ we mean $\operatorname{in}_{\nu}(f)=f_{0}$. Let $G=\left(G_{1}, \ldots, G_{k}\right)$ be an endomorphism of $K\left[\left[x_{1}, \ldots, x_{k}\right]\right.$ such that $x_{i} \mapsto G_{i}$. Then

$$
\operatorname{in}_{\nu}(G):=\left(\operatorname{in}_{\nu}\left(G_{1}\right), \ldots, \operatorname{in}_{\nu}\left(G_{k}\right)\right)
$$

is the endomorphism determined by the initial terms of $G_{i}$.

Lemma 7.2.3. Let $G=\left(G_{1}, \ldots, G_{k}\right)$ be an automorphism of $K\left[\left[x_{1}, \ldots, x_{k}\right]\right]$ preserving a monomial valuation $\nu: G_{*}(\nu)=\nu$. Then $\operatorname{in}_{\nu}(G)$ is an automorphism of $K\left[\left[x_{1}, \ldots, x_{k}\right]\right]$ preserving $\nu$. Moreover for any $f \in K\left[\left[x_{1}, \ldots, x_{k}\right]\right]$ we have $\operatorname{in}_{\nu}(G(f))=\operatorname{in}_{\nu}(G)\left(\operatorname{in}_{\nu}(f)\right)$.

Proof. Write $G_{i}=\operatorname{in}_{\nu}\left(G_{i}\right)+G_{i h}$, where $\nu\left(G_{i h}\right)>\nu\left(G_{i}\right)$.

For any $f \in K\left[\left[x_{1}, \ldots, x_{k}\right]\right]$ write $f=\operatorname{in}_{\nu}(f)+f_{h}$, where $\nu\left(f_{h}\right)>\nu(f)$. Then we have $G(f)=$ $f\left(G_{1}, \ldots, G_{n}\right)=\operatorname{in}_{\nu} f\left(G_{1}, \ldots, G_{n}\right)+f_{h}\left(G_{1}, \ldots, G_{n}\right)$, where by Lemma 7.2.1, $\nu\left(f_{h}\left(G_{1}, \ldots, G_{n}\right)\right)>\nu(f)$.

$\operatorname{in}_{\nu}(f)\left(x_{1}+y_{1}, \ldots, x_{k}+y_{k}\right)$ is a quasihomogeneous polynomial in $x_{1}, \ldots, x_{k}, y_{1}, \ldots, y_{k}$ with weights $\nu\left(x_{1}\right)=\nu\left(y_{1}\right), \ldots, \nu\left(x_{k}\right)=\nu\left(y_{k}\right)$. Write $\operatorname{in}_{\nu} f\left(x_{1}+y_{1}, \ldots, x_{k}+y_{k}\right)=\operatorname{in}_{\nu}(f)\left(x_{1}, \ldots, x_{n}\right)+f_{h}\left(x_{1}, y_{1}, \ldots, x_{n}, y_{n}\right)$ where $f_{h}$ is a quasihomogeneous polynomial in the ideal $\left(y_{1}, \ldots, y_{n}\right) \cdot K\left[\left[x_{1}, \ldots, x_{k}, y_{1}, \ldots, y_{k}\right]\right]$. Applying Lemma 7.2.1 to $x_{i}=\operatorname{in}_{\nu}\left(G_{i}\right)$ and,$y_{i}=G_{i h}$ we obtain

$$
\begin{gathered}
\left.\left(\operatorname{in}_{\nu}(f)\left(G_{1}, \ldots, G_{n}\right)\right)=\operatorname{in}_{\nu}(f)\left(\operatorname{in}_{\nu}\left(G_{1}\right)+G_{1 h}, \ldots, \operatorname{in}_{\nu}\left(G_{n}\right)+G_{n h}\right)\right)= \\
\left.\left.\operatorname{in}_{\nu}(f)\left(\operatorname{in}_{\nu}\left(G_{1}\right), \ldots, \operatorname{in}_{\nu}\left(G_{n}\right)\right)\right)+f_{h}\left(\operatorname{in}_{\nu}\left(G_{1}\right), \ldots, \operatorname{in}_{\nu}\left(G_{n}\right), G_{1 h}, \ldots, G_{n h}\right)\right),
\end{gathered}
$$

where by Lemma 7.2.1, $\nu\left(f_{h}\left(\operatorname{in}_{\nu}\left(G_{1}\right), \ldots, \operatorname{in}_{\nu}\left(G_{n}\right), G_{1 h}, \ldots, G_{n h}\right)\right)>\nu(f)$, which gives

$$
\operatorname{in}_{\nu}(G(f))=\operatorname{in}_{\nu}(G)\left(\operatorname{in}_{\nu}(f)\right) .
$$

Now let $G^{-1}=\left(G_{1}^{\prime}, \ldots, G_{n}^{\prime}\right)$. Then by the above,

$$
\begin{gathered}
G\left(x_{i}\right) \circ G^{-1}\left(x_{i}\right)=G_{i}^{\prime}\left(G_{1}, \ldots, G_{n}\right)=x_{i} \text { and } \\
\operatorname{in}_{\nu}\left(G_{i}^{\prime}\right)\left(\operatorname{in}_{\nu}\left(G_{1}\right), \ldots, \operatorname{in}_{\nu}\left(G_{n}\right)\right)=x_{i} .
\end{gathered}
$$

This shows that $\operatorname{in}_{\nu}(G)$ and $\operatorname{in}_{\nu}\left(G^{-1}\right)$ are automorphisms. They are defined by quasihomogeneous polynomials of $\nu$-degrees $\nu\left(x_{1}\right), \ldots, \nu\left(x_{k}\right)$. It follows from Lemma 7.2.1 that $\nu\left(\operatorname{in}_{\nu}(G(f))\right) \geq \nu(f)$ and $\nu\left(\operatorname{in}_{\nu}\left(G^{-1}(f)\right)\right) \geq$ $\nu(f)$ for any $f \in K\left[\left[x_{1}, \ldots, x_{k}\right]\right]$. Consequently, $\nu\left(\operatorname{in}_{\nu}(G(f))\right)=\nu(f)$.

Lemma 7.2.4. Let $u_{1}, \ldots, u_{n}$ be local semiinvariant parameters on $\widetilde{X}_{\sigma}$. Write $K\left[\widetilde{X}_{\sigma}\right]=K\left[\left[x_{1}, \ldots, x_{k}\right]\right] / I$. Let $\nu$ be a toric valuation on $\widetilde{X}_{\sigma}$ invariant with respect to the group of all automorphisms of $\widetilde{X}_{\sigma}$ centered at $O_{\tilde{\sigma}}$. Then $\nu$ defines a monomial valuation on $K\left[\left[x_{1}, \ldots, x_{k}\right]\right]$ with basis $x_{1}, \ldots, x_{n}$ and weights $\nu\left(u_{1}\right), \ldots, \nu\left(u_{n}\right)$. Moreover:

1. Any automorphism $g$ of $K\left[\widetilde{X}_{\sigma}\right]$ extends to an automorphism $G$ of $K\left[\left[x_{1}, \ldots, x_{k}\right]\right]$ preserving $\nu$ and $I$.

2. The automorphism $\operatorname{in}_{\nu}(G)$ of $K\left[\left[x_{1}, \ldots, x_{k}\right]\right]$ defines an automorphism $\operatorname{in}_{\nu}(g)$ of $K\left[\widetilde{X}_{\sigma}\right]$ preserving $\nu$.

Proof. Any automorphism $g$ of $K\left[\widetilde{X}_{\sigma}\right]$ sends parameters $u_{1}, \ldots, u_{n}$ to $g_{1}=g^{*}\left(u_{1}\right), \ldots, g_{n}=g^{*}\left(u_{n}\right)$. Moreover $\nu\left(g_{i}\right)=\nu\left(u_{i}\right)$ so we may assume that $g_{i}=G_{i}\left(u_{1}, \ldots, u_{n}\right)$ are given by some formal power series $G_{i} \in K\left[\left[x_{1}, \ldots, x_{k}\right]\right]$ of $\nu$-weights $\nu\left(u_{i}\right)$. Let $h=g^{-1}$, and let $h_{1}=h^{*}\left(u_{1}\right), \ldots, h_{n}=h^{*}\left(u_{n}\right)$ be given by some formal power series $H_{i} \in K\left[\left[x_{1}, \ldots, x_{k}\right]\right]$ of $\nu$-weights $\nu\left(u_{i}\right)$. Then $\nu\left(H_{i}\left(G_{1}, \ldots, G_{k}\right)\right) \geq \nu\left(u_{i}\right)$. On the other hand $H_{i}\left(G_{1}\left(u_{1}, \ldots, u_{n}\right), \ldots, G_{k}\left(u_{1}, \ldots, u_{n}\right)\right)=u_{i}$. Hence $\left(H_{i}\left(G_{1}, \ldots, G_{k}\right)-x_{i}\right) \in I$. In other words $H_{i}\left(G_{1}, \ldots, G_{k}\right)=x_{i}+F_{i}\left(x_{1}, \ldots, x_{k}\right)$, where $\nu\left(F_{i}\right) \geq \nu\left(u_{i}\right)$ and $F_{i} \in I$. Consequently, for any $F \in K\left[\left[x_{1}, \ldots, x_{k}\right]\right], \nu(F) \geq \nu\left(F\left(G_{1}, \ldots, G_{k}\right)\right)$.

Let $m \subset K\left[\left[x_{1}, \ldots, x_{k}\right]\right]$ be the maximal ideal. By definition $m /\left(m^{2}+I\right)=m / m^{2}$ and thus $I \subset m^{2}$. This gives $F_{i} \in m^{2}$. Then the ring automorphism $\Phi:=H \circ G$ of $K\left[\left[x_{1}, \ldots, x_{k}\right]\right]$ can be written as a $K$-linear transformation $\Phi=\mathrm{id}+F$, where $F\left(K\left[\left[x_{1}, \ldots, x_{k}\right]\right]\right) \subset I$ and $F\left(m^{i}\right) \subset m^{i+1}$. It follows from Lemma 7.2.1 that for any formal power series $x \in K\left[\left[x_{1}, \ldots, x_{k}\right]\right], \nu(\Phi(x)) \geq \nu(x)$ and hence $\nu(F(x)) \geq$ $\min \{\nu(\phi(x)), \nu(x)\}=\nu(x)$. The inverse of $\Phi$ can be written as $\Phi^{-1}=\mathrm{id}+F^{\prime}$, where $F^{\prime}=-F+F^{2}-F^{3}+\ldots$ By the above $F^{\prime}\left(K\left[\left[x_{1}, \ldots, x_{k}\right]\right]\right) \subset I$ and $\nu\left(F^{\prime}(x)\right) \geq \nu(x)$, which shows that $\nu\left(\Phi^{-1}(x)\right) \geq \nu(x)$ and finally $\nu\left(\Phi^{-1}(x)\right)=\nu(x)$. Hence $G$ and $G^{-1}=\Phi^{-1} \circ H$ are automorphisms satisfying $\nu\left(G^{-1}(x) \geq \nu(x)\right.$. By Lemma 7.2 .1 and the above, $\nu(G(x)) \geq \nu(x)$ for any $x \in K\left[\left[x_{1}, \ldots, x_{k}\right]\right]$. Hence $\nu(G(x))=\nu(x)$. We have shown that any automorphism $g$ of $K\left[\widetilde{\widetilde{X}}_{\sigma}\right]$ is defined by an automorphism $G$ of $K\left[\left[x_{1}, \ldots, x_{k}\right]\right]$ preserving the valuation $\nu$ and the ideal $I$. Then by Lemma 7.2.3, $\operatorname{in}_{\nu}(G)$ is an automorphism of $K\left[\left[x_{1}, \ldots, x_{k}\right]\right]$ preserving the valuation $\nu$ and the ideal $\operatorname{in}_{\nu}(I)=I$. Therefore it defines an automorphism $\operatorname{in}_{\nu}(G)$ of $K\left[\tilde{X}_{\sigma}\right]$. 


\subsection{Structure of the group of automorphisms of the completion of a local ring of a toric variety.}

Lemma 7.3.1. Let $\sigma$ be a $\Gamma$-semicone. Let $\nu$ be the valuation on $\widetilde{X}_{\sigma}$ associated to the absolutely invariant vector $v_{\sigma}$. Let $G_{\nu}^{\sigma}:=\operatorname{Aut}_{\nu}\left(\widetilde{X}_{\sigma}\right)$ denote the group of all quasihomogeneous automorphisms with respect to semiinvariant coordinates on $\widetilde{X}_{\sigma}$ with weights detrmined by $\nu$. Then

1. $G_{\nu}^{\sigma}$ is an algebraic group.

2. There is a surjective morphism $\Phi: \operatorname{Aut}\left(\widetilde{X}_{\sigma}\right) \rightarrow \operatorname{Aut}_{\nu}\left(\widetilde{X}_{\sigma}\right)$ defined by taking the lowest degree quasihomogeneous part.

3. The kernel $\operatorname{ker}_{\sigma}$ of $\Phi$ is a connected proalgebraic group.

4. $\operatorname{Aut}\left(\widetilde{X}_{\sigma}\right)=G_{\nu}^{\sigma} \cdot \operatorname{ker}_{\sigma}$.

5. For any $g \in \operatorname{ker}_{\sigma}$ there exists a morphism $i_{g}: \mathbf{A}^{1} \rightarrow \operatorname{ker}_{\sigma}$ such that $g=i_{g}(1)$, id $=i_{g}(0)$.

6. For any $g \in \operatorname{Aut}\left(\widetilde{X}_{\sigma}\right)^{0}$ there exists a morphism $i_{g}: W:=\mathbf{A}^{1} \times\left(G_{\nu}^{\sigma}\right)^{0} \rightarrow \operatorname{Aut}\left(\widetilde{X}_{\sigma}\right)^{0}$ such that $\{g, \mathrm{id}\} \subset$ $i_{g}(W)$.

Proof. Let $u_{1}, \ldots, u_{k}$ be semiinvariant parameters on $\widetilde{X}_{\sigma}$ generating the maximal ideal of $O_{\tilde{\sigma}}$. Denote by $m_{1}, \ldots, m_{k} \in \widetilde{\sigma}$ the corresponding characters.

(1) Let $\operatorname{Aut}_{\nu}\left(\mathbf{A}^{k}\right)$ denote the group of all quasihomogeneous automorphisms of $\mathbf{A}^{k}$. By Lemma 7.2.4, $\operatorname{Aut}_{\nu}\left(\widetilde{X}_{\sigma}\right)$ is isomorphic to the subgroup of $\operatorname{Aut}_{\nu}\left(\mathbf{A}^{k}\right)$ consisting of the automorphisms preserving $I$. The automorphisms of $\operatorname{Aut}_{\nu}\left(\mathbf{A}^{k}\right)$ are described by sets of $k$ quasihomogeneous polynomials with weights $\nu\left(u_{1}\right), \ldots, \nu\left(u_{k}\right)$ and having linearly independent linear terms. Thus $\operatorname{Aut}_{\nu}\left(\mathbf{A}^{k}\right)$ and $\operatorname{Aut}_{\nu}\left(\widetilde{X}_{\sigma}\right)$ are algebraic.

(2) This follows from Lemma 7.2.1 and 7.2.4

(3)(4)(5) The 1-parameter subgroup $t \mapsto t^{\nu_{\sigma}}$ defines the action on $\widetilde{X}_{\sigma}$ such that $\psi_{t}\left(u_{i}\right)=t^{\left(m_{i}, v_{\sigma}\right)} u_{i}=$ $t^{\nu\left(u_{i}\right)} u_{i}=t^{\alpha_{i}}$, where $\alpha_{i}=\nu\left(u_{i}\right)$ is a $\nu$-weight of $u_{i}$. The action defines an embedding $K^{*}<\operatorname{Aut}\left(\widetilde{X}_{\sigma}\right)$.

Write $g \in \operatorname{ker}_{\sigma}$ in terms of coordinate functions, $g=\left(g_{1}, \ldots, g_{k}\right)$, where $g_{i}=g^{*}\left(u_{i}\right)$. Let $\alpha_{i, 0}=\alpha_{i}$ denote the $\nu$-weight of $g_{i}$ and let $g_{i}=u_{i}+g_{\alpha_{i, 1}}+\ldots+g_{\alpha_{i, j}}+\ldots$ be the decomposition according to $\nu$-weights $\alpha_{i, 0}<\alpha_{i, 1}<\ldots<\alpha_{i, j}<\ldots$. Define the morphism $i_{g}: K^{*} \rightarrow \operatorname{ker}_{\sigma}$ by $i_{g}(t):=\psi_{t}^{-1} \circ g \circ \psi_{t}$. Then $\left(i_{g}(t)\right)^{*}\left(u_{i}\right)=\left(\psi_{t}^{-1} \circ g \circ \psi_{t}\right)^{*}\left(u_{i}\right)=\left(g \circ \psi_{t}\right)^{*} t^{-\alpha_{i}}\left(u_{i}\right)=\psi_{t}^{*}\left(t^{-\alpha_{i}} g_{i}\right)=t^{-\alpha_{i}}\left(t^{\alpha_{i, 0}} u_{i}+t^{\alpha_{i, 1}} g_{\alpha_{i, 1}}+\ldots+\right.$ $\left.t^{\alpha_{i, j}} g_{\alpha_{i, j}}+\ldots\right)==u_{i}+t^{\alpha_{i, 1}-\alpha_{i, 0}} g_{\alpha_{i, 1}}+\ldots+t^{\alpha_{i, j}-\alpha_{i, 0}} g_{\alpha_{i, j}}+\ldots$. The above morphism extends to a morphism $i_{g}: \mathbf{A}^{1} \rightarrow \operatorname{ker}_{\sigma}$ "connecting" id to $g$. This shows that $\operatorname{ker}_{\sigma}$ is connected. Note that $\Psi$ maps the connected component $\operatorname{Aut}_{\nu}\left(\widetilde{X}_{\sigma}\right)^{0}$ to the connected component $\operatorname{Aut}\left(\widetilde{X}_{\sigma}\right)^{0}$.

(6) Write $g \in \operatorname{Aut}\left(\tilde{X}_{\sigma}, S\right)^{0}$ as $g=g_{1} g_{2}$, where $g_{1} \in \operatorname{ker}_{\sigma}, g_{2} \in G_{\nu}^{\sigma}$. Then for $t \in \mathbf{A}^{1}$ and $h \in G_{\nu}^{\sigma}$, set $i_{g}(t, h):=i_{g_{1}}(t) \cdot h$.

Lemma 7.3.2. (22])) Let $\sigma$ be a $\Gamma$-semicone. Then $\operatorname{Aut}\left(\widetilde{X}_{\sigma}\right)^{0} \subset \operatorname{Aut}\left(\widetilde{X}_{\sigma}\right)$ is a normal subgroup and there is a natural surjection $\operatorname{Aut}(\sigma) \longrightarrow \operatorname{Aut}\left(\widetilde{X}_{\sigma}\right) / \operatorname{Aut}\left(\widetilde{X}_{\sigma}\right)^{0}$.

Proof. Set $G:=\operatorname{Aut}_{\nu}\left(\widetilde{X}_{\sigma}\right)=\operatorname{Aut}_{\nu}\left(X_{\widetilde{\sigma}}\right)$ (notation of Lemma 7.3.1). It follows from Lemma 7.3.1 that the natural inclusion $G \subset \operatorname{Aut}\left(\widetilde{X}_{\sigma}\right)$ determines a group isomorphism $G / G^{0} \simeq \operatorname{Aut}\left(\widetilde{X}_{\sigma}\right) /\left(\operatorname{Aut}\left(\widetilde{X}_{\sigma}\right)\right)^{0}$. Then we get a surjection $N_{G}(T) / T \rightarrow G / G^{0}$. Let $H:=\operatorname{Aut}_{T}\left(X_{\sigma}\right)$ be the group of the $\Gamma$-equivariant automorphisms of $X_{\sigma}$, preserving the big torus $T$. It suffices to show that $N_{G}(T)=H$ and $H / T \simeq \operatorname{Aut}(\sigma)$. If $g \in N_{G}(T)$ then $g T g^{-1}=T$. Let $x \in T \subset X_{\tilde{\sigma}}$. Then $g T g^{-1} x=T x$ or equivalently $T g^{-1} x=g^{-1} T x$. Since the latter subset is open, $g^{-1} x$ is in the big open orbit $T g^{-1} x=T x$, in other words, $g^{-1} x \in T$ which shows that $g \in H$ and $N_{G}(T) \subset H$. Let $H^{0}$ be the connected component of $H$ containing id. Then $H_{0}$ acts trivially on irreducible components of the complement of $T$, that is, on $T$-invariant Weil and Cartier divisors. Hence it multiplies characters by invertible functions on $X_{\tilde{\sigma}}$. Invertible functions on $T$ are monomials. Invertible monomials on $X_{\widetilde{\sigma}}$ are constants. Thus $H_{0}$ acts on characters multiplying them by nonzero constants. This shows that $H_{0}=T$. Hence for any $g \in H, g T g^{-1}=T$, which shows that $N_{G}(T) \supset H . H / T$ can be identified with the subgroup of $H$ preserving $1 \in T \subset X_{\tilde{\sigma}}$, that is, the automorphisms mapping characters to characters. The latter subgroup is equal to the $\operatorname{group} \operatorname{Aut}(\widetilde{\sigma})$ of automorphisms of embedded semifan $\sigma \subset \widetilde{\sigma}$.

The restriction of an automorphism in $\operatorname{Aut}(\widetilde{\sigma})$ is an automorphism of $\sigma$. Any automorphism of $\sigma$ defines an automorphism of $\widetilde{\sigma}=\sigma \times \operatorname{reg}(\sigma)$. Thus there are natural group homomorphisms $i: \operatorname{Aut}(\sigma) \rightarrow \operatorname{Aut}(\widetilde{\sigma})$ and $p: \operatorname{Aut}(\widetilde{\sigma}) \rightarrow \operatorname{Aut}(\sigma)$ such that $p \circ i=\operatorname{id}_{\sigma}$. Moreover $p$ corresponds to the restriction of automorphisms of $X_{\widetilde{\sigma}}$ to the subvariety $X_{\sigma} \times O_{\operatorname{reg}(\sigma)} \subset X_{\widetilde{\sigma}}$. By Lemma 4.9.14, $p\left(\operatorname{Aut}(\widetilde{\sigma})^{0}=\operatorname{Aut}(\sigma)^{0}\right.$. 


\subsection{Invariant and semiinvariant valuations.}

Lemma 7.4.1. Let $v \in \sigma$ be an integral vector. Then $\operatorname{val}(v)$ is an $\operatorname{Aut}\left(\widetilde{X}_{\sigma}\right)^{0}$-invariant valuation on $\widetilde{X}_{\sigma}$ iff it is $\operatorname{Aut}\left(\widetilde{X}_{\sigma}\right)$-semiinvariant.

Proof. $(\Leftarrow)$. If $\operatorname{val}(v)$ is $\operatorname{Aut}\left(\widetilde{X}_{\sigma}\right)$-semiinvariant then it is $\operatorname{Aut}\left(\widetilde{X}_{\sigma}\right)^{0}$-semiinvariant. Hence by Lemma 5.3.15 $(1)$ it is $\operatorname{Aut}\left(\widetilde{X}_{\sigma}\right)^{0}$-invariant. $(\Rightarrow)$. If $\operatorname{val}(v)$ is $\operatorname{Aut}\left(\widetilde{X}_{\sigma}\right)$-invariant then by Lemma 7.3.2, Aut $\left(\widetilde{X}_{\sigma}\right)$ acts on $\operatorname{val}(v)$ as the finite group $\operatorname{Aut}(\sigma) \longrightarrow \operatorname{Aut}\left(\widetilde{X}_{\sigma}\right) / \operatorname{Aut}\left(\widetilde{X}_{\sigma}\right)^{0}$. Therefore $\operatorname{val}(v)$ is $\operatorname{Aut}\left(\widetilde{X}_{\sigma}\right)$-semiinvariant.

Lemma 7.4.2. Let $L$ be any algebraically closed field containing $K$. Then $\operatorname{val}(v)$ defines $\operatorname{Aut}\left(\widetilde{X}_{\sigma}\right)^{0}$-invariant valuation on $\widetilde{X}_{\sigma}$ iff it defines an $\operatorname{Aut}_{L}\left(\widetilde{X}_{\sigma}\right)^{0}$-invariant valuation on $\widetilde{X}_{\sigma}^{L}$.

Proof. Follows from Lemma $4.5 .3(3)$.

For a $\Gamma$-semicone $\sigma$ denote by $\widehat{\operatorname{Inv}}(\sigma)$ the cone generated by vectors $v \in|\sigma|$, for which valuations $\operatorname{val}\left(v, \widehat{X}_{\sigma}\right)$ are invariant with respect to $\operatorname{Aut}\left(\widehat{X}_{\sigma}\right)^{0}$.

Lemma 7.4.3. For a $\Gamma$-semicone $\sigma$ the two cones are equal $\widehat{\operatorname{Inv}}(\sigma)=\operatorname{Inv}(\sigma)$.

Proof. Note that $K\left[\widetilde{X}_{\sigma}\right]=L\left[\widehat{X}_{\sigma}\right]$ for suitable $L:=K\left[\left[x_{1}, \ldots, x_{k}\right]\right]$. Let $(L)$ denote the algebraic closere of the quotient field of $L$. Each automorphism of $K\left[\left[\sigma^{\vee}\right]\right]$ defines the automorphism of $L\left[\left[\sigma^{\vee}\right]\right]$ which is constant on $L$. Each automorphism in ${ }^{\vee} \operatorname{Aut}\left(\widehat{X}_{\sigma}\right)^{0}$ determines an automorphism in $\operatorname{Aut}\left(\widetilde{X}_{\sigma}\right)^{0}$, so that there is a monomorphism of proalgebraic groups $\imath: \operatorname{Aut}\left(\widehat{X}_{\sigma}\right)^{0} \rightarrow \operatorname{Aut}\left(\widetilde{X}_{\sigma}\right)^{0}$. Therefore if $\operatorname{val}\left(v, \widetilde{X}_{\sigma}\right)$ is invariant with respect to $\operatorname{Aut}\left(\widetilde{X}_{\sigma}\right)^{0}$ then it is $\imath\left(\operatorname{Aut}\left(\widehat{X}_{\sigma}\right)^{0}\right)$-invariant. Since the subring $K\left[\widehat{X}_{\sigma}\right] \subset K\left[\widetilde{X}_{\sigma}\right]$ is $\imath\left(\operatorname{Aut}\left(\widehat{X}_{\sigma}\right)^{0}\right)$ invariant then the restriction $\operatorname{val}\left(v, \widehat{X}_{\sigma}\right)$ of $\operatorname{val}\left(v, \widetilde{X}_{\sigma}\right)$ to $\widehat{X}_{\sigma}$ is $\operatorname{Aut}\left(\widehat{X}_{\sigma}\right)^{0}$-invariant.

Now, if $\operatorname{val}\left(v, \widehat{X}_{\sigma}\right)$ is $\operatorname{Aut}\left(\widehat{X}_{\sigma}\right)^{0}$-invariant then by Lemma 7.4.2, it defines an $\operatorname{Aut}_{(L)}\left(\widehat{X}_{\sigma}^{(L)}\right)^{0}$-invariant valuation. By Lemma 7.4.1, $\operatorname{val}\left(v, \widehat{X}_{\sigma}^{(L)}\right)$ is $\operatorname{Aut}_{(L)}\left(\widehat{X}_{\sigma}^{(L)}\right)$-semiinvariant. The proalgebraic group Aut ${ }_{L}\left(\widetilde{X}_{\sigma}\right)=$ $\operatorname{Aut}_{L}\left(\widehat{X}_{\sigma}^{L}\right)$ is a subgroup (as an abstract group) of $\operatorname{Aut}_{(L)}\left(\widehat{X}_{\sigma}^{(L)}\right)$. Thus the restriction $\operatorname{val}\left(v, \widetilde{X}_{\sigma}\right)$ of $\operatorname{val}\left(v, \widehat{X}_{\sigma}^{(L)}\right)$ is $\operatorname{Aut}_{L}\left(\widetilde{X}_{\sigma}\right)$-semiinvariant. By Lemma 4.7.1 any automorphism $\phi$ in $\operatorname{Aut}\left(\widetilde{X}_{\sigma}\right)$ can be $\operatorname{decomposed}$ as $\phi=\phi_{0} \phi_{1}$, where $\phi_{0}$ preserves monomials and $\phi_{1} \in \operatorname{Aut}_{L}\left(\widetilde{X}_{\sigma}\right)$. Therefore $\operatorname{val}\left(v, \widetilde{X}_{\sigma}\right)$ is $\operatorname{Aut}\left(\widetilde{X}_{\sigma}\right)$-semiinvariant and consequently by Lemma 7.4 .1 it is $\operatorname{Aut}\left(\widetilde{X}_{\sigma}\right)^{0}$-invariant.

Lemma 7.4.4. If $\sigma \leq \tau$ then

1. $\operatorname{Inv}(\sigma) \subset \operatorname{Inv}(\tau)$.

2. $\operatorname{stab}(\sigma) \subset \operatorname{stab}(\tau) \cap \sigma$.

Proof. (1) Let $v \in \operatorname{Inv}(\sigma)$. Any automorphism $\phi \in \operatorname{Aut}\left(\widetilde{X}_{\tau}\right)$ preserves the $\operatorname{stratum} \operatorname{strat}(\sigma)$ and thus defines an automorphism $\hat{\phi}$ of the completion of $Y:=\widetilde{X}_{\tau}$ at $\operatorname{strat}(\sigma)$ which by Lemma 4.7 .3 is isomorphic to the spectrum of the ring $K\left[\widehat{Y}_{\text {strat }(\sigma)}\right]=K_{\sigma}\left[\widehat{X}_{\sigma}\right]$, where $K_{\sigma}$ is the residue field of the generic orbit point $O_{\sigma}$. By Lemma 4.7.1 any automorphism $\widehat{\phi}$ of $K_{\sigma}\left[\widehat{X}_{\sigma}\right]$ can be written as $\widehat{\phi}=\phi_{0} \phi_{1}$, where $\phi_{0}$ preserves monomials and $\phi_{1} \in \operatorname{Aut}_{K_{\sigma}}\left(\widehat{X}_{\sigma}\right)$. By Lemma 7.4.3, $\operatorname{val}\left(v, \widehat{X}_{\sigma}\right)$ is Aut $\left(\widehat{X}_{\sigma}\right)^{0}$-invariant. Let $\overline{K_{\sigma}}$ be the algebraic closure of $K_{\sigma}$. By Lemma 7.4.2, $\operatorname{val}\left(v, \widehat{X}_{\sigma}^{\bar{K}_{\sigma}}\right)$ is $\operatorname{Aut}_{K_{\sigma}}\left(\widehat{X}_{\sigma}^{\bar{K}_{\sigma}}\right)^{0}$-invariant. By Lemma 7.4.1, $\operatorname{val}\left(v, \widehat{X}_{\sigma}^{\overline{K_{\sigma}}}\right)$ is $\operatorname{Aut}_{K_{\sigma}}\left(\widehat{X}_{\sigma}^{K_{\sigma}}\right)$ semiinvariant. Then its restriction $\operatorname{val}\left(v, \widehat{X}_{\sigma}^{K_{\sigma}}\right)$ is $\operatorname{Aut}_{K_{\sigma}}\left(\widehat{X}_{\sigma}^{K_{\sigma}}\right)$-semiinvariant. Thus $\phi_{1 *}\left(\operatorname{val}\left(v, \widehat{X}_{\sigma}^{K_{\sigma}}\right)\right)$ can be one of the finitely many toric valuations on $\widehat{X}_{\sigma}^{K_{\sigma}}$ for any $\phi_{1} \in \operatorname{Aut}_{K_{\sigma}}\left(\widehat{X}_{\sigma}\right)$. But then $\widehat{\phi}_{*}\left(\operatorname{val}\left(v, \widehat{Y}_{\text {strat }(\sigma)}\right)\right)=$ $\phi_{1 *}\left(\operatorname{val}\left(v, \widehat{Y}_{\text {strat }(\sigma)}\right)\right)$ can be one of the finitely many toric valuations on $K\left[\widehat{Y}_{\text {strat }(\sigma)}\right]$ for all $\phi \in \operatorname{Aut}\left(\widetilde{X}_{\tau}\right)$. The restrictions of these valuations to the local ring of $\widetilde{X}_{\tau}$ at strat $(\sigma)$ define finitely many valuations on $\widetilde{X}_{\tau}$. Hence $\operatorname{val}\left(v, \widetilde{X}_{\tau}\right)$ is semiinvariant and consequently invariant on $\widetilde{X}_{\tau}$. (2) Follows from (1) and from Lemma 6.1 .2 .

\subsection{Group of divisor classes of the completion of a local ring of a toric variety.}

Lemma 7.5.1. Let $\sigma$ be a cone in a lattice $N$ and $\Delta$ be a suddivision $\sigma$. Let $\widehat{X}_{\Delta}:=X_{\Delta} \times_{X_{\sigma}} \widehat{X}_{\sigma}$. The following groups of divisor classes are isomorphic. (The isomorphisms are determined by the natural morphisms).

1. $\operatorname{Cl}\left(\widehat{X}_{\sigma}\right) \simeq \operatorname{Cl}\left(X_{\sigma}\right), \operatorname{Pic}\left(\widehat{X}_{\sigma}\right) \simeq \operatorname{Pic}\left(X_{\sigma}\right)$.

2. $\operatorname{Cl}\left(\widehat{X}_{\Delta}\right) \simeq \operatorname{Cl}\left(X_{\Delta}\right), \operatorname{Pic}\left(\widehat{X}_{\Delta}\right) \simeq \operatorname{Pic}\left(X_{\Delta}\right)$. 
3. For the affine toric variety $X_{\sigma}$ set $\mathbf{A}^{1} \widehat{\times} \widehat{X}_{\sigma}:=\lim _{\rightarrow} \mathbf{A}^{1} \times X_{\sigma}^{(n)}=\operatorname{Spec}\left(K[t]\left[\widehat{X}_{\sigma}\right]\right)$. Then $\mathrm{Cl}\left(\mathbf{A}^{1} \widehat{\times} \widehat{X}_{\sigma}\right) \simeq \operatorname{Cl}\left(\mathbf{A}^{1} \times X_{\sigma}\right) \simeq \operatorname{Cl}\left(X_{\sigma}\right), \operatorname{Pic}\left(\mathbf{A}^{1} \widehat{\times} \widehat{X}_{\sigma}\right) \simeq \operatorname{Pic}\left(\mathbf{A}^{1} \times X_{\sigma}\right) \simeq \operatorname{Pic}\left(X_{\sigma}\right)$.

4. For any subdivision $\Delta$ of $\sigma$ set $\mathbf{A}^{1} \widehat{\times} \widehat{X}_{\Delta}:=\left(\mathbf{A}^{1} \widehat{\times} \widehat{X}_{\sigma}\right) \times_{X_{\sigma}} X_{\Delta}$. Then $\mathrm{Cl}\left(\mathbf{A}^{1} \widehat{\times} \widehat{X}_{\Delta}\right) \simeq \operatorname{Cl}\left(\mathbf{A}^{1} \times \widehat{X}_{\Delta}\right) \simeq \operatorname{Cl}\left(X_{\Delta}\right), \operatorname{Pic}\left(\mathbf{A}^{1} \widehat{\times} \widehat{X}_{\Delta}\right) \simeq \operatorname{Pic}\left(\mathbf{A}^{1} \times \widehat{X}_{\Delta}\right) \simeq \operatorname{Pic}\left(X_{\Delta}\right)$.

Proof. Let $v_{1}, \ldots, v_{k}$ denote the generators of $\sigma \cap N_{\sigma}$ and let $\tau=\left\langle e_{1}, \ldots, e_{k}\right\rangle$ denote the regular $k$ dimensional cone. Let $\pi: \tau \rightarrow \sigma$ be the projection defined by $\pi\left(e_{i}\right)=v_{i}$. Then $\pi$ defines a surjective morphism of lattices $\pi: N_{\tau} \rightarrow N_{\sigma}$ whose kernel is a saturated sublattice $N \subset N_{\tau}$. Thus the projection $\pi$ defines the quotient map $X_{\tau} \rightarrow X_{\sigma}=X_{\tau} / / T$ for the subtorus $T \subset T_{\tau}$ corresponding to the sublattice $N \subset N_{\tau}$. This also defines the quotient morphism $\alpha: \widehat{X}_{\tau} \rightarrow \widehat{X}_{\sigma}$, where $K\left[\widehat{X}_{\sigma}\right]=K\left[\widehat{X}_{\tau}\right]^{T}$.

Denote by $T_{\tau} \subset X_{\tau}, T_{\sigma} \subset X_{\sigma}$ the relevant tori and set $\widehat{T}_{\tau}:=\widehat{X}_{\tau} \times_{X_{\tau}} T_{\tau} \subset \widehat{X}_{\tau}, \widehat{T}_{\sigma}:=\widehat{X}_{\sigma} \times_{X_{\sigma}} T_{\sigma} \subset \widehat{X}_{\sigma}$. Then both schemes are nonsingular and let $\alpha^{\prime}: \widehat{T}_{\tau} \rightarrow \widehat{T}_{\sigma}$ be the restriction of $\alpha$. We also have $K\left[\widehat{T}_{\sigma}\right]=$ $K\left[\widehat{T}_{\tau}\right]^{T}$.

The morphism $\alpha$ defines a group homomorphism $\operatorname{Cl}\left(\widehat{T}_{\sigma}\right)=\operatorname{Pic}\left(\widehat{T}_{\sigma}\right) \stackrel{\alpha^{*}}{\longrightarrow} \operatorname{Pic}\left(\widehat{T}_{\tau}\right)=\operatorname{Cl}\left(\widehat{T}_{\tau}\right)=0$. The last group is 0 since the ring $K\left[\widehat{X}_{\tau}\right]$ as well as its localization $K\left[\widehat{T}_{\tau}\right]$ are UFD. Let $D$ be an effective Cartier divisor on $\widehat{T}_{\sigma}$. Then $\alpha^{*}(D)$ is $T$ - invariant and principal on $\widehat{T}_{\tau}$. This means that the ideal $I$ of $\alpha^{*}(D)$ is generated by $f \in K\left[\widehat{T}_{\tau}\right]$. By multiplying by a suitable monomial we can asume that $f \in K\left[\widehat{X}_{\tau}\right]^{T}$. Note that all $t \cdot f, t \in T$ also belong to $I$. Let $M_{T}$ denote the lattice of characters of $T$. Let $f_{\beta}$ denote the component of $f$ with weight $\beta \in M_{T}$. By considering $K\left[\widehat{X}_{\tau}\right] / m^{k}$, where $m \subset K\left[\widehat{X}_{\tau}\right]$ is the maximal ideal, we see that all $f_{\beta}+m^{k} \in I \cdot K\left[\widehat{X}_{\tau}\right] / m^{k}$. Moreover $I \cdot K\left[\widehat{X}_{\tau}\right] / m^{k}$ is generated by all $f_{\beta}+m^{k}$. This yields $I=\left(f_{\beta}\right)_{\beta \in M_{T}}$ or $(1)=\left(f_{\beta} / f\right)$ showing that there is a $\beta_{0}$ such that $f_{\beta_{0}} / f$ is invertible, which means $I=\left(f_{\beta_{0}}\right)$. By multiplying by monomials we can assume that $f_{\beta_{0}}$ is $T$-invariant. Thus $f_{\beta_{0}} \in K\left[\widehat{T}_{\sigma}\right]^{T}=K\left[\widehat{X}_{\tau}\right]$ and generates the ideal of $D$. But then $D$ is principal on $\widehat{X}_{\tau}$ and finally $\mathrm{Cl}\left(\widehat{T}_{\sigma}\right)=0$.

Now any divisor $D$ in $\operatorname{Cl}\left(\widehat{X}_{\sigma}\right)$ (respectively in $\operatorname{Cl}\left(\widehat{X}_{\Delta}\right)$ ) is linearly equivalent to the $T$-invariant one $D^{\prime}:=D-\left(f_{D}\right)$, where $D_{\mid \widehat{T}_{\sigma}}=\left(f_{D}\right)_{\mid \widehat{T}_{\sigma}}$.

(3)(4) We repeat the reasoning from (1) and (2) and use the fact that $K\left[\mathbf{A}^{1} \widehat{\times} \widehat{X}_{\tau}\right]=K[t]\left[\left[x_{1}, \ldots, x_{k}\right]\right]$ is UFD (see Bourbaki[11]).

Lemma 7.5.2. 1. $G^{\sigma}$ acts trivially on $\operatorname{Cl}\left(\widetilde{X}_{\Delta^{\sigma}}\right)$.

2. Let $\Delta^{\sigma}$ be a subdivision of $\sigma$ such that $\widetilde{X}_{\Delta^{\sigma}} \rightarrow \widetilde{X}_{\sigma}$ is $G^{\sigma}$-equivariant. Then $G^{\sigma}$ acts trivially on $\operatorname{Cl}\left(\widetilde{X}_{\Delta^{\sigma}}\right)$.

Proof. We shall use the notation and results from Lemma 7.3.1.

(1) The natural morphism $\widetilde{X}_{\Delta^{\sigma}} \rightarrow X_{\Delta^{\sigma}}$ is $G_{\nu}^{\sigma}$-equivariant. By Lemma 7.5 .1 this morphism induces a $G_{\nu}^{\sigma}$-equivariant isomorphism $\mathrm{Cl}\left(\widetilde{X}_{\sigma}\right) \rightarrow \mathrm{Cl}\left(X_{\sigma}\right)$. By Sumihiro [67 the algebraic linear group acts trivially on $\mathrm{Cl}\left(X_{\sigma}\right)$, which yields a trivial action on $\mathrm{Cl}\left(\widetilde{X}_{\sigma}\right)$. By Lemma 7.3.1 it suffices to show that $\mathrm{ker}_{\sigma}$ acts trivially on $\operatorname{Cl}\left(\widetilde{X}_{\sigma}\right)$.

Let $\widetilde{X}_{\sigma}^{\text {ns }} \subset \widetilde{X}_{\sigma}$ denote the open subset of nonsingular points. Its complement is of codimension 2. We get a $G^{\sigma}$-equivariant isomorphism $\operatorname{Pic}\left(\widetilde{X}_{\sigma}^{\text {ns }}\right)=\operatorname{Cl}\left(\widetilde{X}_{\sigma}^{\text {ns }}\right) \simeq \mathrm{Cl}\left(\widetilde{X}_{\sigma}\right)$. It suffices to prove that $\operatorname{ker}_{\sigma}$ acts trivially on $\operatorname{Pic}\left(\widetilde{X}_{\sigma}^{\text {ns }}\right)$. Fix $g \in G$. Let $i_{g, \mathbf{A}^{1}}: \mathbf{A}^{1} \rightarrow \operatorname{ker}_{\sigma}$ be the morphism from Lemma 7.3.1. Let $\Phi: G^{\sigma} \widehat{\times} \widetilde{X}_{\sigma}^{\text {ns }} \rightarrow \widetilde{X}_{\sigma}^{\text {ns }}$ be the action morphism from Lemma 4.5.1 and set $\Phi_{\mathbf{A}^{1}}:=i_{g, \mathbf{A}^{1}} \circ \Phi: \mathbf{A}^{1} \widehat{\times} \widetilde{X}_{\sigma}^{\text {ns }} \rightarrow \widetilde{X}_{\sigma}^{\text {ns }}$. Denote by $p: \mathbf{A}^{1} \widehat{\times} \widetilde{X}_{\sigma}^{\mathrm{ns}} \rightarrow \widetilde{X}_{\sigma}^{\mathrm{ns}}$ the standard projection. By Lemma 7.5.1 $(4), p^{*}: \operatorname{Pic}\left(\widetilde{X}_{\sigma}^{\mathrm{ns}}\right) \rightarrow \operatorname{Pic}\left(\mathbf{A}^{1} \widehat{\times} \widetilde{X}_{\sigma}^{\mathrm{ns}}\right)$ is an isomorphism . Let $D \in \operatorname{Pic}\left(\widetilde{X}_{\sigma}^{\text {ns }}\right)$. Let $j_{g}: \widetilde{X}_{\sigma}^{\text {ns }} \rightarrow\{g\} \widehat{\times} \widetilde{X}_{\sigma}^{\text {ns }} \subset \mathbf{A}^{1} \widehat{\times} \widetilde{X}_{\sigma}^{\text {ns }}$ denote the standard embedding. Then $p \circ j_{g}=\operatorname{id}_{\mid \widetilde{X}_{\sigma}^{\text {ns }}}$. Since $p^{*}$ is an isomorphism there is $D^{\prime} \in \operatorname{Pic}\left(\widetilde{X}_{\sigma}^{\text {ns }}\right)$ such that $\Phi_{\mathbf{A}^{1}}^{*}(D) \simeq p^{*}\left(D^{\prime}\right)$. Therefore $j_{g}^{*} \Phi_{\mathbf{A}^{1}}^{*}(D)=g \cdot D \simeq j_{g}^{*} p^{*}\left(D^{\prime}\right)=D^{\prime}$. This implies $h \cdot D \simeq D$ for any $h \in G=\operatorname{ker}_{\sigma} \cdot \operatorname{Aut}_{\nu}\left(\widetilde{X}_{\sigma}\right)$

(2) Let $L \subset \widetilde{X}_{\Delta^{\sigma}}$ denote the complement of the set where the birational morphism $\psi: \widetilde{X}_{\Delta^{\sigma}} \rightarrow \widetilde{X}_{\sigma}$ is an isomorphism. It is a $G^{\sigma}$-invariant subset of codimension 2. Therefore we get $G^{\sigma}$-equivariant homomorphisms $\mathrm{Cl}\left(\widetilde{X}_{\sigma}\right) \simeq \mathrm{Cl}\left(\widetilde{X}_{\sigma} \backslash L\right) \hookrightarrow \mathrm{Cl}\left(\widetilde{X}_{\Delta^{\sigma}}\right)$. Note also that $\mathrm{Cl}\left(\widetilde{X}_{\Delta^{\sigma}}\right)$ is generated by $\mathrm{Cl}\left(\widetilde{X}_{\sigma}\right)$ and by the exceptional divisors of $\psi$ which are $G^{\sigma}$-invariant by Lemma 5.3 .10 . Finally, all elements of $\mathrm{Cl}\left(\widetilde{X}_{\Delta^{\sigma}}\right)$ are $G^{\sigma}$-invariant.

7.6. Simple definition of a canonical subdivision of a semicomplex. Recall that for any fan $\Sigma$ we denote by $\operatorname{Vert}(\Sigma)$ the set of all 1-dimensional rays in $\Sigma$. 
Proposition 7.6.1. Let $\Sigma$ be an oriented $\Gamma$-semicomplex. A subdivision $\Delta$ of $\Sigma$ is canonical if for any $\sigma \in \Sigma$

$$
\operatorname{Vert}\left(\Delta^{\sigma}\right) \backslash \operatorname{Vert}(\bar{\sigma}) \subset \operatorname{Stab}(\Sigma) \text {. }
$$

Proof. This is an immediate consequence of Lemma 6.1.2 and the following.

Proposition 7.6.2. Let $\sigma$ be a $\Gamma$-semicone and $\Delta^{\sigma}$ be a subdivision of $\sigma$. Then the following conditions are equivalent:

1. $\widetilde{X}_{\Delta^{\sigma}} \rightarrow \widetilde{X}_{\sigma}$ is $G^{\sigma}$-equivariant (where $\left.G^{\sigma}=\operatorname{Aut}\left(\widetilde{X}_{\sigma}\right)^{0}\right)$.

2. $\operatorname{Vert}\left(\Delta^{\sigma}\right) \subset \operatorname{Vert}(\bar{\sigma}) \cup \operatorname{Inv}(\sigma)$.

3. $\widehat{X}_{\Delta^{\sigma}} \rightarrow \widehat{X}_{\sigma}$ is $\operatorname{Aut}\left(\widehat{X}_{\sigma}\right)^{0}$-equivariant.

Before the proof of Propositions 7.6.2 and 7.6.1 we need to show a few lemmas below.

Lemma 7.6.3. Let $\sigma$ be a $\Gamma$-semicone. If $\Delta_{1}$ and $\Delta_{2}$ are subdivisions of $\sigma$ for which $\widetilde{X}_{\Delta_{i}} \rightarrow \widetilde{X}_{\sigma}$ is $G^{\sigma}$ equivariant then for the subdivision

$$
\Delta_{1} \cdot \Delta_{2}:=\left\{\sigma_{1} \cap \sigma_{2} \mid \sigma_{1} \in \Delta_{1}, \sigma_{2} \in \Delta_{2}\right\}
$$

the morphism $\widetilde{X}_{\Delta_{1} \cdot \Delta_{2}} \rightarrow \widetilde{X}_{\sigma}$ is $G^{\sigma}$-equivariant.

Let $I_{1}$ and $I_{2}$ be $G^{\sigma}$-invariant on $\widetilde{X}_{\sigma}$. Let $\Delta_{1}$ and $\Delta_{2}$ be subdivisions of $\sigma$ corresponding to the normalized blow-ups at $I_{1}$ and $I_{2}$. Then $I_{1} \cdot I_{2}$ corresponds to $\Delta_{1} \cdot \Delta_{2}$.

Proof. It follows from the universal property of the fiber product that $\Delta_{1} \cdot \Delta_{2}$ corresponds to the normalization of an irreducible component in $\widetilde{X}_{\Delta_{1}} \times_{\widetilde{X}_{\sigma}} \widetilde{X}_{\Delta_{2}}$.

The second part of the lemma is an immediate consequence of the relations between ideals and ordfunctions: $\operatorname{ord}\left(I_{1} \cdot I_{2}\right)=\operatorname{ord}\left(I_{1}\right)+\operatorname{ord}\left(I_{2}\right)$ corresponds to $\Delta_{1} \cdot \Delta_{2}([\sqrt{39}])$.

Lemma 7.6.4. Let $\sigma$ be a $\Gamma$-semicone. Let $\tau \subset|\sigma|$ be a cone with all rays $\operatorname{Vert}(\tau)$ in $\operatorname{Vert}(\bar{\sigma}) \cup \operatorname{Inv}(\sigma)$.

1. There exists a fan subdivision $\Delta_{\tau}$ of $\sigma$ which contains $\tau$ as its face and for which $\widetilde{X}_{\Delta^{\tau}} \rightarrow \widetilde{X}_{\tau}$ is $G^{\sigma}$-equivariant.

2. If all rays of $\tau$ which are not in $\operatorname{Inv}(\sigma)$ determine a face $\varrho$ of $|\sigma|$ (and $\tau$ ) then there exists a $G^{\sigma}$-invariant ideal I such that the normalized blow-up of I corresponds to the subdivision $\Delta_{\tau}$ of $\sigma$ containing $\tau$.

Proof. Write $\tau=\left\langle v_{1}, \ldots, v_{l}\right\rangle$, where $v_{1}, \ldots, v_{k} \in \operatorname{Inv}(\sigma)$ and $v_{k+1}, \ldots, v_{l}$ are in $\operatorname{Vert}(\bar{\sigma})$.

(1) Let $\left\{\tau_{i} \mid i \in J_{0}\right\}$ denote the set of all one-codimensional faces of $\tau$. For any one-codimensional face $\tau_{i}$ of $\tau$, where $i \in J_{0}$, find an integral functional $H_{i}$ such that $H_{i \mid \tau_{i}}=0$ and $H_{i \mid \tau \backslash \tau_{i}}>0$. Find functionals $H_{j}$, $j \in J_{1}$ with common zeros exactly on $\operatorname{lin}(\tau)$. Then $\tau=\left\{x \in \sigma \mid H_{i}(x) \geq 0, H_{j}(x)=0, i \in J_{0}, j \in J_{1}\right\}$.

Let $F_{0}$ be an integral functional such that for any $i \in J_{0}$ and $j \in J_{1}, F_{0}+H_{i}$ and $F_{0}-\left|H_{j}\right|$ are strictly greater than zero on $\sigma \backslash\{0\}$. Set $F_{0}\left(v_{1}\right)=n_{1}, \ldots, F_{0}\left(v_{l}\right)=n_{l}$. Let $\nu_{1}, \ldots, \nu_{l}$ be valuations corresponding to $v_{1}, \ldots, v_{l}$. Set

$$
I:=I_{\nu_{1}, n_{1}} \cap \ldots \cap I_{\nu_{l}, n_{l}} .
$$

Let $p: \widetilde{X}_{\Delta_{0}} \rightarrow \widetilde{X}_{\sigma}$ be the composition of the $G^{\sigma}$-equivariant blow-ups bl $\nu_{\nu_{k}} \circ \ldots \circ \mathrm{bl}_{\nu_{1}}$ corresponding to $G^{\sigma}$-invariant valuations $\nu_{1}, \ldots, \nu_{k}$. Then all valuations $\nu_{1}, \ldots, \nu_{l}$ correspond to Weil divisors $D_{1}, \ldots, D_{l}$ on $\widetilde{X}_{\Delta_{0}}$. Set $D=n_{1} D_{1}+\ldots+n_{l} D_{l}$. Then

$$
p_{*}\left(I_{D}\right)=\left\{f \in p_{*}\left(\mathcal{O}_{\tilde{X}_{\Delta_{\tau}}}\right)=\mathcal{O}_{\tilde{X}_{\sigma}} \mid \nu_{i}(f) \geq n_{i}, i=1, \ldots, l\right\}=I .
$$

By Lemma 7.5.2, $G^{\sigma}$ acts trivially on $\mathrm{Cl}\left(\widetilde{X}_{\Delta_{0}}\right)$; then for any $g \in G$ we have $g_{*}(D)=D+\left(f_{g}\right)-\left(h_{g}\right)$, where $f_{g}, h_{g} \in \mathcal{O}_{\tilde{X}_{\sigma}}$. In other words for any $g \in G$,

$$
f_{g} \cdot I_{D}=h_{g} \cdot I_{g_{*}(D)},
$$

which implies

$$
f_{g} \cdot I=h_{g} \cdot g_{*}(I) .
$$

This means that the action of $G^{\sigma}$ lifts to the blow-up of $I$, and to its normalization. The normalized blow-up of $I$ corresponds to a $G^{\sigma}$-equivariant subdivision $\Delta_{\tau}$ of $\sigma$ into maximal cones, where the piecewise linear function $\operatorname{ord}(I)=\min \left\{F \in \sigma^{\vee} \mid F\left(v_{i}\right) \geq n_{i}\right\}$ is linear. We have to show that $\tau \in \Delta_{\tau}$. Note that $\operatorname{ord}(I)$ is linear on $\tau$ since by definition $\operatorname{ord}(I)_{\mid \tau}=F_{0 \mid \tau}$.

Let $x \notin \tau$. Consider first the case $x \in \operatorname{lin}(\tau)$. There exists a one-codimensional face $\tau_{i_{0}}, i_{0} \in J_{0}$, of $\tau$ such that $H_{i_{0}}(x)<0$. Then $F_{1}:=F_{0}+H_{i_{0}} \in \sigma^{\vee}$ and by definition $F_{1} \geq \operatorname{ord}(I)$, since $H_{i_{0}}\left(v_{i}\right) \geq 0$ and 
$F_{1}\left(v_{i}\right)=F_{0}\left(v_{i}\right)+H_{i_{0}}\left(v_{i}\right) \geq n_{i}$. This implies ord $(I)(x) \leq F_{1}(x)<F_{0}(x)$. Now let $x \notin \operatorname{lin}(\tau)$. Then there is $H_{j_{0}}, j_{0} \in J_{1}$ such that $H_{j_{0}}(x) \neq 0$. Set $F_{1}:=F_{0}+H_{j_{0}}$ if $H_{j_{0}}(x)<0$ and $F_{1}:=F_{0}-H_{j_{0}}$ otherwise. The definition of $F_{0}$ shows that $F_{1}$ is strictly greater than zero on $\sigma \backslash\{0\}$. We have $F_{1}\left(v_{i}\right)=F_{0}\left(v_{i}\right)+H_{j_{0}}\left(v_{i}\right)=n_{i}$ and consequently ord $(I) \leq F_{1}$. Again $\operatorname{ord}(I)(x) \leq F_{1}(x)<F_{0}(x)$. This implies $\operatorname{ord}(I)<F_{0}$ off $\operatorname{lin}(\tau)$.

(2) The reasoning is very similar. Let $J_{0}$ denote the set of all one-codimensional faces $\tau_{i}$ of $\tau$ which contain $\varrho$. For any $i \in J_{0}$ find an integral functional $H_{i}$ such that $H_{i \mid \tau_{i}}=0$ and $H_{i \mid \tau \backslash \tau_{i}}>0$. Let $H_{j}, j \in J_{1}$, be functionals with common zeros exactly on $\operatorname{lin}(\tau)$. Then $\tau=\left\{x \in \sigma \mid H_{i}(x) \geq 0, H_{j}(x)=0, i \in J_{0}, j \in J_{1}\right\}$.

Let $F_{0}$ be an integral functional such that $F_{0 \mid \tau}=0$, and for any $i \in I$ and $j \in J, F_{0}+H_{i}, F_{0}-\left|H_{j}\right|$ are strictly greater than zero on $\sigma \backslash \varrho$ for $j \in J_{1}$. Set $F_{0}\left(v_{1}\right)=n_{1}, \ldots, F_{0}\left(v_{k}\right)=n_{k}$. Then $F_{0}\left(v_{k+1}\right)=F_{0}\left(v_{l}\right)=0$. Let $\nu_{1}, \ldots, \nu_{k}$ be valuations corresponding to $v_{1}, \ldots, v_{k}$. Set

$$
I:=I_{\nu_{1}, n_{1}} \cap \ldots \cap I_{\nu_{k}, n_{k}} .
$$

By definition $I$ is $G^{\sigma}$-invariant. The normalization of the blow-up of $I$ corresponds to a $G^{\sigma}$-equivariant subdivision $\Delta_{\tau}$ of $\sigma$ into maximal cones, where the piecewise linear function ord $(I)=\min \left\{F \in \sigma^{\vee} \mid F\left(v_{i}\right) \geq\right.$ $\left.n_{i}\right\}$ is linear. We have to show that $\tau \in \Delta_{\tau}$. Note that $\operatorname{ord}(I)$ is linear on $\tau$ since by definition $\operatorname{ord}(I)_{\mid \tau}=F_{0 \mid \tau}$.

The rest of the proof is the same as in (1).

Lemma 7.6.5. Let $\sigma$ be a $\Gamma$-semicone and $\tau \subset|\sigma|$ be a cone all of whose rays are in $\operatorname{Vert}(\bar{\sigma}) \cup \operatorname{Inv}(\sigma)$. Let $\Delta_{\tau}$ be a subdivision of $\sigma$ containing $\tau$ as its face and for which $\widetilde{X}_{\Delta_{\tau}} \rightarrow \widetilde{X}_{\sigma}$ is $G^{\sigma}$-equivariant. Then

$$
S\left(\tau, \Delta_{\tau}\right):=\left\{\varrho \in \Delta_{\tau} \mid(\varrho \backslash \tau) \cap \operatorname{Inv}(\sigma)=\emptyset\right\}
$$

is a subfan of $\Delta_{\tau}$ for which the open subset $\tilde{X}_{S\left(\tau, \Delta_{\tau}\right)}=X_{S\left(\tau, \Delta_{\tau}\right)} \times_{X_{\sigma}} \tilde{X}_{\sigma}$ of $\tilde{X}_{\Delta_{\tau}}$ is $G^{\sigma}$-invariant.

Proof It suffices to show that each $G^{\sigma}$-orbit intersecting $\widetilde{X}_{S\left(\tau, \Delta_{\tau}\right)}:=X_{S\left(\tau, \Delta_{\tau}\right)} \times_{X_{\sigma}} \widetilde{X}_{\sigma}$ is contained in this subscheme. Since $\widetilde{X}_{S\left(\tau, \Delta_{\tau}\right)}$ is an open subset of $\widetilde{X}_{\Delta_{\tau}}$ any $G^{\sigma}$-orbit contains the generic point of $O_{\varrho}$ for $\varrho \in S\left(\tau, \Delta_{\tau}\right)$. By Lemma 5.3.15 (3), $\operatorname{int}(\varrho)$ intersects $\operatorname{Inv}(\sigma)$. The other toric orbits in the $G^{\sigma}$-orbit correspond to the cones $\varrho^{\prime} \in \operatorname{Star}(\varrho, \Sigma)$ for which $\varrho^{\prime} \backslash \varrho=\emptyset$. Thus $\varrho$ is a face of $\tau$ and all $\varrho^{\prime}$ belong to $S\left(\tau, \Delta_{\tau}\right)$.

Lemma 7.6.6. Let $\sigma$ be a $\Gamma$-semicone. Then $S(\tau):=S(\tau, \Delta)$ does not depend upon a subdivision $\Delta$ of $\sigma$ containing $\tau$ and all of whose rays are in $\operatorname{Vert}(\bar{\sigma}) \cup \operatorname{Inv}(\sigma)$.

Proof. Let $\Delta_{1}$ and $\Delta_{2}$ be two subdivisions containing $\tau$ and for which all rays are $\operatorname{in} \operatorname{Vert}(\bar{\sigma}) \cup \operatorname{Inv}(\sigma)$. By Lemmas 7.6.3 and 7.6.4 we may find subdivisions $\Delta_{\varrho}$, where $\varrho \in \Delta_{1} \cup \Delta_{2}$ and the common subdivision $\Delta:=\prod_{\varrho \in \Delta_{1} \cup \Delta_{2}} \Delta_{\varrho}$ such that $\widetilde{X}_{\Delta} \rightarrow \widetilde{X}_{\sigma}$ is $G^{\sigma}$-equivariant. Then $\operatorname{Vert}\left(\Delta_{1} \cdot \Delta_{2}\right) \subset \operatorname{Vert}(\Delta) \subset \operatorname{Vert}(\sigma) \cup \operatorname{Inv}(\sigma)$. Hence we can assume that $\Delta_{1}$ is a subdivision of $\Delta_{2}$ replacing $\Delta_{1}$ with $\Delta_{1} \cdot \Delta_{2}$ if necessary. Let $\varrho \in S\left(\tau, \Delta_{2}\right)$. By Lemma 6.5.1 (1) applied to $\Delta_{\varrho}$ we can represent $\varrho$ as $\varrho:=\varrho^{\prime} \oplus\left\langle e_{1}, \ldots, e_{k}\right\rangle$, where $\operatorname{int}\left(\varrho^{\prime}\right) \cap \operatorname{Inv}(\sigma) \neq \emptyset$, $\left(\varrho \backslash \varrho^{\prime}\right) \cap \operatorname{Inv}(\sigma)=\emptyset$ and thus $\varrho^{\prime} \preceq \tau$. Since all new rays of $\Delta_{1} \mid \varrho$ are in $\operatorname{Inv}(\sigma)$ we find that $\Delta_{1} \mid \varrho=$ $\Delta_{1} \mid \varrho^{\prime} \oplus\left\langle e_{1}, \ldots, e_{k}\right\rangle$. But $\Delta_{1} \mid \varrho^{\prime}=\varrho^{\prime}$ since $\varrho^{\prime} \preceq \tau$. Therefore $\Delta_{1} \mid \varrho=\varrho$ and $\varrho \in \Delta_{1}$, and consequently $\varrho \in S\left(\tau, \Delta_{1}\right)$.

Lemma 7.6.7. Let $\sigma$ be a $\Gamma$-semicone. Let $\Delta$ be any subdivision of $\sigma$ with all "new " rays belonging to $\operatorname{Inv}(\sigma)$. Then for any $\tau \in \Delta, \widetilde{X}_{S(\tau)}$ is $G^{\sigma}$-invariant.

Proof By Lemma 7.6.4, we find a subidvision $\Delta_{\tau}$ of $\sigma$. By Lemma 7.6.6, $\widetilde{X}_{S(\tau)}=\widetilde{X}_{S\left(\tau, \Delta_{\tau}\right)}$. By Lemma 7.6.5, the latter scheme is $G^{\sigma}$-invariant.

Proof of Proposition 7.6.2. (1) $\Rightarrow(2)$ All exceptional divisors determine $G^{\sigma}$-semiinvariant and therefore $G^{\sigma}$-invariant valuations. The latter correspond to vectors in $\operatorname{Vert}(\Sigma) \subset \operatorname{Vert}(\sigma)$.

$(1) \Leftarrow(2)$ Let $\Delta$ be any subdivision of $\sigma$ containing $\tau$ with all "new " rays belonging to $\operatorname{Inv}(\sigma)$. Then by Lemmas 7.6.7, $\widetilde{X}_{\Delta}$ is the union of open $G^{\sigma}$-invariant neighborhoods $\widetilde{X}_{S(\tau)}$, where $\tau \in \Delta$, and consequently, $\widetilde{X}_{\Delta}$ is $G^{\sigma}$-invariant.

$(2) \equiv(3)$ By Lemma 7.4 .3 vectors defining $G^{\sigma}$-invariant valuations are the same as those defining $\operatorname{Aut}\left(\widehat{X}_{\sigma}\right)^{0}$-invariant valuations. Then the proof of the equivalence is the same as for $(1) \equiv(2)$. We have to replace $\widetilde{X}_{\sigma}$ with $\widehat{X}_{\sigma}$. 
Lemma 7.6.8. Let $\sigma$ be a $\Gamma$-semicone and $G^{\sigma}$ be a connected proalgebraic group acting on $X:=\tilde{X}_{\sigma}$. Let $\Delta^{\sigma}$ be a subdivision of $\sigma$ such that $G^{K}$ acts on $Y:=\widetilde{X}_{\Delta^{\sigma}}$ and the toric morphism $\psi: Y \rightarrow X$ is a proper birational $G^{K}$-equivariant morphism. Then there exists a toric ideal $I$ on $X$ such that the normalization of the blow-up of $I, Z \rightarrow X$, factors as $Z \rightarrow Y \rightarrow X$.

Proof. First subdivide $\Delta^{\sigma}$ so that all its faces satisfy the condition of Lemma 7.6.4 (2). We can make $\Delta^{\sigma}$ simplicial by applying additional star subdivisions at all its 1 -dimensional rays (see 72 ). Let $\tau$ be a face of $\Delta^{\sigma}$ which does not satisfy the condition of Lemma 7.6.4(2). Denote by $\tau^{\prime}$ a minimal face of $\tau$ with rays in $\operatorname{Vert}(\bar{\sigma})$ and which is not a face of the cone $\sigma$. Then there is $\sigma^{\prime}$ which is a face of $\sigma$ and for which $\operatorname{int}\left(\tau^{\prime}\right) \subset \operatorname{int}\left(\sigma^{\prime}\right)$. Write $\sigma^{\prime}=\operatorname{sing}^{\Gamma}\left(\sigma^{\prime}\right) \oplus^{\Gamma}\left\langle e_{1}, \ldots, e_{k}\right\rangle$. Then $\tau^{\prime} \cap \operatorname{sing}^{\Gamma}\left(\sigma^{\prime}\right)$ is a face of $\tau^{\prime}$ but it is not a face of $\sigma$. (Otherwise $\tau^{\prime}$ was a face of $\sigma$.) By minimality $\tau^{\prime}=\tau^{\prime} \cap \operatorname{sing}^{\Gamma}\left(\sigma^{\prime}\right) \subset \operatorname{sing}^{\Gamma}\left(\sigma^{\prime}\right)$ and $\sigma^{\prime}=\operatorname{sing}^{\Gamma}\left(\sigma^{\prime}\right)$ is indecomposable and since $\operatorname{int}\left(\tau^{\prime}\right) \subset \operatorname{int}\left(\sigma^{\prime}\right)$ we can find by Lemma 5.3.15 (5) a vector $v \in \operatorname{int}(\tau) \cap \operatorname{Inv}(\sigma)$. By applying star subdivision at $\langle v\rangle$ to $\Sigma$ we eliminate the cone $\tau$ (and some others cones not satisfying the condition of Lemma 7.6.4(2)). After a finite number of steps we arrive at a subdivision $\Delta^{\sigma}$ with all faces satisfying the condition of Lemma 7.6.4(2).

Then by Lemma 7.6.4 (2), for any $\tau \in \Delta$, we construct the ideal $I_{\tau}$ such that the normalization of the blow-up of $I_{\tau}$ corresponds to the subdivision $\Delta_{\tau}^{\sigma}$ of $\sigma$ containing $\tau$. Then by Lemma 7.6.3 the $G^{\sigma}$-invariant ideal $I:=\prod_{I_{\tau} \in \Sigma} I_{\tau}$ correponds to the subdivision $\Delta_{I}:=\prod_{\tau \in \Sigma} \Delta_{\tau}^{\sigma}$ of $\sigma$.

Lemma 7.6.9. Let $\sigma$ be a face of a fan $\Sigma$. Let $\Delta_{1}$ be a subdivision of $\sigma$ such that $\operatorname{Vert}\left(\Delta_{1}\right)=\operatorname{Vert}(\sigma)$. Then there is a subdiision $\Delta_{2}$ of $\Sigma$ such that $\Delta_{2} \mid \sigma=\Delta_{1}$ and $\operatorname{Vert}\left(\Delta_{2}\right)=\operatorname{Vert}(\Sigma)$.

Proof. Set $\left\{v_{1}, \ldots, v_{k}\right\}=\operatorname{Vert}(\Sigma) \backslash \operatorname{Vert}(\sigma), \Delta_{0}:=\left\langle v_{1}\right\rangle \cdots\left\langle v_{k}\right\rangle \cdot \Sigma$. Then $\sigma \in \Delta_{0}$. We shall construct a subdivision $\Delta_{2}$ for any cone in $\Delta_{0}$. If $\tau \in \Delta_{0}$ does not intersect $\sigma$ then we put $\Delta_{2} \mid \tau=\tau$. If $\tau \in \Delta_{0}$ intersects $\sigma$ along a common face $\sigma_{0}$ then all rays $\operatorname{Vert}\left(\tau \backslash \sigma_{0}\right)$ are centers of star subdivisions and therefore are linearly independent of the other rays of $\sigma$ and in particular of $\operatorname{lin}\left(\sigma_{0}\right)$ (After a star subdivision at $\langle v\rangle, v$ becomes linearly independent of all other rays of cones containing $v$. Hence the subdivision $\Delta_{2} \mid \sigma_{0}$ extends to a unique subdivision of $\tau$ with no new rays, $\Delta_{2} \mid \tau:=\left\{\delta+\sum_{\varrho \in\left(\operatorname{Vert}(\tau) \backslash \operatorname{Vert}\left(\sigma_{0}\right)\right)} \varrho|\delta \in \Delta| \sigma_{0}\right\}$.

Lemma 7.6.10. Let $\tau \subset \sigma$ be a cone such that $\operatorname{Vert}(\tau) \subset \operatorname{Vert}(\sigma)$. Then there is a proper subdivision $\Delta$ of $\sigma$ containing $\tau$ and such that $\operatorname{Vert}(\Delta)=\operatorname{Vert}(\sigma)$.

Proof. By Lemma 7.6.9 it suffices to prove the lemma for the situation when $\operatorname{Vert}(\sigma) \backslash \operatorname{Vert}(\tau)$ consists of one ray. Then for any $\tau$ we find $\sigma^{\prime}$ containing $\tau$ and for which $\operatorname{card}(\operatorname{Vert}(\sigma) \backslash \operatorname{Vert}(\tau))=1$. By induction we can find a subdivision of $\sigma^{\prime}$ and then by Lemma 7.6.9 extend it to a subdivision of $\sigma$.

Let $\tau=\left\langle v_{1}, \ldots, v_{k}\right\rangle$ and $\sigma=\left\langle v_{1}, \ldots, v_{k}, v_{k+1}\right\rangle$. If $\tau$ is simplicial and $\tau$ is not a face of $\sigma$ then there is a unique linear relation between $v_{1}, \ldots, v_{k}, v_{k+1}$. Without loss of generality we can assume that it is $a_{r} v_{r}+$ $a_{r+1} v_{r+1}+\ldots+a_{l} v_{l}=a_{l+1} v_{l+1}+\ldots+a_{k+1} v_{k+1}$, where $r \geq 1$ and all coefficients are positive. Then there are exactly two simplicial subdivisions of $\sigma$ with no "new" rays and with maximal cones $\left\langle v_{1}, \ldots, \check{v}_{i}, \ldots, v_{k}, v_{k+1}\right\rangle$, where $i=r, \ldots l$ and $i=l+1, \ldots, k+1$ (see [72]). The second subdivision contains $\tau$. If $\tau=\left\langle v_{1}, \ldots, v_{k}\right\rangle$ is not simplicial then for the cone $\tau^{\vee}$ we find a simplicial cone $\left(\tau_{0}^{\vee}, M_{\tau_{0}}\right)$ and a linear epimorphism $\phi^{\vee}$ : $\left(\tau_{0}^{\vee}, M_{\tau_{0}} \rightarrow\left(\tau^{\vee}, M_{\tau}\right)\right.$ mapping rays of $\tau_{0}^{\vee}$ to rays of $\tau_{0}^{\vee}$. Moreover let $H^{\vee} \subset M_{\tau_{0}}$ denote the vector subspace which is the kernel of $\phi^{\vee}$. Then $\tau^{\vee} \simeq\left(\tau_{0}^{\vee}+H^{\vee}\right) / H^{\vee}$. The dual morphism $\phi:\left(\tau, N_{\tau}\right) \rightarrow\left(\tau_{0}, N_{\tau_{0}}\right)$ is a monomorphism which maps $\tau$ isomorphically to $\tau_{0} \cap H$, where $H=\left\{v \in N_{\tau_{0}} \mid v_{\mid H^{\vee}}=0\right\}$. Then also $v_{k+1} \in H \backslash \tau_{0}$. Note that $v_{k+1} \notin-\tau_{0}$ since otherwise $v_{k+1} \in\left(-\tau_{0}\right) \cap H=-\tau$. Thus we can apply the previous case to $\sigma_{0}:=\left\langle v_{k+1}\right\rangle+\tau_{0} \supset \tau_{0}$ and obtain a subdivision $\Delta_{0}$ of $\sigma_{0}$ containing $\tau_{0}$ with no "new " rays. Intersecting $\Delta_{0}$ with $H$ defines a subdivision $\Delta$ of $\sigma$ containing $\tau$. All rays in $\Sigma$ except $v_{k+1} \in H$ are obtained by intersecting some faces of $\tau_{0}$ with $H$, hence belong to $\operatorname{Vert}(\tau)$.

\section{ORIENTABILITY OF STRATIFIED TOROIDAL VARIETIES AND RESOLUTION OF SINGULARITIES}

\subsection{Orientation group of an affine toric variety.}

Definition 8.1.1. Let $(X, S)$ be a stratified noetherian scheme over $K$ and $x \in X$ be a $K$-rational point fixed under the $\Gamma$-action. By the orientation group of $(X, S)$ at $x \in X$ we mean $\Theta^{\Gamma}(X, S, x):=\operatorname{Aut}\left(\widehat{X}_{X, x}\right) / \operatorname{Aut}\left(\widehat{X}_{X, x}\right)^{0}$.

Lemma 8.1.2. Let $\sigma$ be a $\Gamma$-semicone. Let $\widehat{\operatorname{Inv}}(\sigma)=\operatorname{Inv}(\sigma)$ be the set of the vectors $v \in \sigma$ corresponding to $\operatorname{Aut}\left(\widehat{X}_{\sigma}\right)^{0}$-invariant valuations $\operatorname{val}(v)$ on $\widehat{X}_{\sigma}$ (see Lemmma 7.4.S). Let $\operatorname{Aut}(\sigma)_{\text {Inv }}$ be the group of all elements $g$ of $\operatorname{Aut}(\sigma)$ satisfying the following conditions: 
1. $g_{\mid \operatorname{Inv}(\sigma)}=\mathrm{id}_{\mid \operatorname{Inv}(\sigma)}$.

2. For any subdivision $\Delta$ of $\sigma$ such that $\operatorname{Vert}(\Delta)=\operatorname{Vert}(\bar{\sigma}), g$ lifts to an automorphism of $\Delta$.

Then $\operatorname{Aut}(\sigma)_{\mathrm{Inv}}=\operatorname{Aut}(\sigma)^{0}$ and $\Theta^{\Gamma}\left(X_{\sigma}, S, O_{\sigma}\right)=\operatorname{Aut}(\sigma) / \operatorname{Aut}(\sigma)_{\mathrm{Inv}}$.

Proof. By Lemma 7.3.2 there is an epimomorphism Aut $(\sigma) \longrightarrow \Theta^{\Gamma}\left(X_{\sigma}, S, O_{\sigma}\right)$ with kernel Aut $(\sigma)^{0}$. By definition all $g \in \operatorname{Aut}(\sigma)^{0}$ preserve $\operatorname{Inv}(\sigma)$. By Proposition 7.6.1 the subdivisions $\Delta$ satisfying condition (2) of the lemma determine Aut $\left(\widehat{X}_{\sigma}\right)^{0}$-equivariant morphisms $\widehat{X}_{\Delta} \rightarrow \widehat{X}_{\sigma}$ which commute with morphisms defined by $g \in \operatorname{Aut}(\sigma)^{0}$. Thus automorphisms $g \in \operatorname{Aut}(\sigma)^{0}$ lift to automorphisms of $\Delta$. Therefore Aut $(\sigma)^{0} \subseteq$

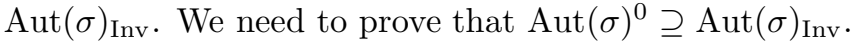

Let $v_{1}, \ldots, v_{k}$ denote the generators of $\sigma$. Let $\sigma_{0}$ denote the regular cone spanned by the standard basis $e_{1}, \ldots, e_{k}$ of some $k$-dimensional lattice. Let $\pi: \sigma_{0} \rightarrow \sigma$ be the projection defined by $\pi\left(e_{i}\right)=$ $v_{i}$. Each automorphism of $\sigma$ lifts to a unique automorphism of $\sigma_{0}$. This defines a group homomorphism $\pi^{*}: \operatorname{Aut}(\sigma)_{\operatorname{Inv}} \rightarrow \operatorname{Aut}\left(\sigma_{0}\right)$. Let $\operatorname{Aut}\left(\sigma_{0}\right)_{\operatorname{Inv}}$ denote the group of automorphisms of the cone $\sigma_{0}$ preserving $\pi^{-1}(\operatorname{Inv}(\sigma))$.

Lemma 8.1.3. $\operatorname{Aut}\left(\sigma_{0}\right)_{\mathrm{Inv}} \simeq \operatorname{Aut}(\sigma)_{\mathrm{Inv}}$.

Proof. Any automorphism $g \in \operatorname{Aut}(\sigma)_{\text {Inv }}$ preserves all vectors from $\operatorname{Inv}(\sigma)$ and consequently it preserves the vectors from $\overline{\operatorname{Inv}(\sigma)}:=\operatorname{lin}(\operatorname{Inv}(\sigma)) \cap \sigma=\operatorname{lin}(\overline{\operatorname{Inv}(\sigma)}) \cap \sigma \cdot \operatorname{Set} \overline{\operatorname{Inv}\left(\sigma_{0}\right)}:=\pi^{-1}(\overline{\operatorname{Inv}(\sigma)})=\left\langle w_{1}, \ldots, w_{m}\right\rangle$. Then, by definition $\overline{\operatorname{Inv}\left(\sigma_{0}\right)}=\operatorname{lin}\left(\overline{\operatorname{Inv}\left(\sigma_{0}\right)}\right) \cap \sigma_{0}$.

Let $g^{\prime}:=\pi^{*}(g)$. We shall show that $g^{\prime}$ preserves $\overline{\operatorname{Inv}\left(\sigma_{0}\right)}$.

Let $w_{i} \in \operatorname{int}\left(\tau_{i}\right)$, where $\tau_{i}=\left\langle e_{i j_{1}}, \ldots, e_{i j_{l}}\right\rangle \preceq \sigma_{0}$. Then , by definition, $w_{i}$ defines a unique ray of the cone $\tau_{i} \cap \overline{\operatorname{Inv}\left(\sigma_{0}\right)}=\tau_{i} \cap \operatorname{lin}\left(\overline{\operatorname{Inv}\left(\sigma_{0}\right)}\right)$. Then $\operatorname{ker}(\pi) \subset \operatorname{lin}\left(\overline{\operatorname{Inv}\left(\sigma_{0}\right)}\right)$ and $\operatorname{ker}(\pi) \cap \operatorname{lin}\left(\tau_{i}\right)=\operatorname{ker}(\pi) \cap \operatorname{lin}\left(\overline{\operatorname{Inv}\left(\sigma_{0}\right)}\right) \cap$ $\operatorname{lin}\left(\tau_{i}\right)=\operatorname{ker}(\pi) \cap \operatorname{lin}\left\{w_{i}\right\}=0$. Consequently $\pi$ maps $\tau_{i}$ isomorphically onto a cone $\pi\left(\tau_{i}\right)=\left\langle v_{i j_{1}}, \ldots, v_{i j_{l}}\right\rangle$ containing a unique ray $\pi\left(w_{i}\right) \in \overline{\operatorname{Inv}(\sigma)}$. By Lemma 7.6.10 such a cone is a face of some subdivision $\Delta$ of $\sigma$ satisfying condition (2) of the lemma. Thus $\pi\left(\tau_{i}\right)$ is a unique face of $\Delta$ containing $\pi\left(w_{i}\right)$ in its interior. Therefore it is preserved by $g$. Consequently, $g^{\prime}$ preserves $\tau_{i}$ and $w_{i}$, and hence all points from $\overline{\operatorname{Inv}\left(\sigma_{0}\right)}$.

Now, let $g^{\prime}$ be an automorphism of $\sigma_{0}$ preserving $\overline{\operatorname{Inv}\left(\sigma_{0}\right)}$. Then $g^{\prime}$ defines a permutation $g$ of $v_{1}, \ldots, v_{k}$.

We will show that such a permutation preserves linear relations between $v_{1}, \ldots, v_{k}$.

Let $p:=a_{i_{1}} v_{i_{1}}+\ldots+a_{i_{r}} v_{i_{r}}=a_{i_{r+1}} v_{i_{r+1}}+\ldots+a_{i s} v_{i_{s}}$ be a minimal linear relation, i.e. $s-1=$ $\operatorname{dim}\left\{v_{i_{1}}, \ldots, v_{i_{s}}\right\}$. Then both cones $\sigma_{1}:=\left\langle v_{i_{1}}, \ldots, v_{i_{r}}\right\rangle$ and $\sigma_{2}:=\left\langle v_{i_{r+1}}, \ldots, v_{i_{\sigma}}\right\rangle$ are simplicial and by Lemma 7.6 .10 they are faces of two subdivisions $\Delta_{1}$ and $\Delta_{2}$ of $\sigma$ satisfying the condition 2 of the lemma. Then $\langle p\rangle \in \operatorname{Vert}\left(\Delta_{1} \cdot \Delta_{2}\right)$ and by Lemma 7.6.3 and Proposition 7.6.2, $p \in \operatorname{Inv}(\sigma)$. Consequently the point $p^{\prime}:=a_{i_{1}} e_{i_{1}}+\ldots+a_{i_{r}} e_{i_{r}} \in \overline{\operatorname{Inv}\left(\sigma_{0}\right)}$. This implies $p^{\prime}=g^{\prime}\left(p^{\prime}\right)=a_{i_{1}} g^{\prime}\left(e_{i_{1}}\right)+\ldots+a_{i_{r}} g^{\prime}\left(e_{i_{r}}\right)$ and $p=a_{i_{1}} v_{i_{1}}+\ldots+a_{i_{r}} v_{i_{r}}=a_{i_{1}} g\left(v_{i_{1}}\right)+\ldots+a_{i_{r}} g\left(v_{i_{r}}\right)$. Analogously $p=a_{i_{r+1}} g\left(v_{i_{r+1}}\right)+\ldots+a_{i s} g\left(v_{i_{s}}\right)$. Finally, $a_{i_{1}} g\left(v_{i_{1}}\right)+\ldots+a_{i_{r}} g\left(v_{i_{r}}\right)=a_{i_{r+1}} g\left(v_{i_{r+1}}\right)+\ldots+a_{i s} g\left(v_{i_{s}}\right)$ and $g$ defines a linear automorphism preserving vectors from $\overline{\operatorname{Inv}(\sigma)}$.

We need to show that $g$ satisfies condition (2). Let $\Delta$ be a subdivision satisfying (2). If $\tau$ is any face of $\Delta$ whose relative interior intersects $\operatorname{Inv}(\sigma) \subset \overline{\operatorname{Inv}(\sigma)}$ then it lifts to a face $\tau^{\prime}$ of $\sigma_{0}$ whose relative interior intersect $\overline{\operatorname{Inv}\left(\sigma_{0}\right)}$. Thus $\sigma^{\prime}$ is preserved by $g^{\prime}$ and $\sigma$ is preserved by $g$. Therefore all faces of $\Delta$ whose relative interior intersect $\operatorname{Inv}(\sigma)$ are preserved. If the relative interior of $\tau \in \Delta$ does not intersect $\operatorname{Inv}(\sigma)$ then $\tau \in S\left(\sigma^{\prime}\right) \subset \Delta$, where $\sigma^{\prime} \in \Delta$, is the maximal face of $\tau$ whose relative interior intersects $\operatorname{Inv}(\sigma)(\operatorname{see}$ Lemma 7.6.5). By the above, $\sigma^{\prime}$ is preserved by $g$. Thus by 7.6.6 $g(\tau) \in S\left(\sigma^{\prime}, g(\Delta)\right)=S\left(\sigma^{\prime}, \Delta\right)=S\left(\sigma^{\prime}\right)$. Consequently, all faces of $\Delta$ are mapped to faces of $\Delta$ and finally $g$ defines an automorphism of $\Delta$. Lemma 8.1 .3 is proven.

Write $X_{\sigma}=X_{\sigma_{0}} / / T_{0}$ for the torus $T_{0}$ corresponding to the kernel of $\pi$. Also $\widehat{X}_{\sigma}=\widehat{X}_{\sigma_{0}} / / T_{0}$ and $K\left[\widehat{X}_{\sigma}\right]=K\left[\widehat{X}_{\sigma_{0}}\right]^{T_{0}}$.

Let $\operatorname{Aut}\left(\widehat{X}_{\sigma_{0}}\right)_{\text {Inv }}$ denote the group of all $T_{0}$-equivariant automorphisms $g$ in $\operatorname{Aut}\left(\widehat{X}_{\sigma_{0}}\right)$ which preserve invariant valuations, i.e $g_{*}(\operatorname{val}(v))=\operatorname{val}(v)$ for any $v \in \overline{\operatorname{Inv}\left(\sigma_{0}\right)}$. By definition each automorphism $g \in$ $\operatorname{Aut}\left(\widehat{X}_{\sigma_{0}}\right)_{\text {Inv }}$ defines an automorphism of $K\left[\widehat{X}_{\sigma_{0}}\right]^{T_{0}}$. This gives the homomorphism $p: \operatorname{Aut}\left(\widehat{X}_{\sigma_{0}}\right)_{\text {Inv }} \longrightarrow$ $\operatorname{Aut}\left(\widehat{X}_{\sigma}\right)$. It now suffices to prove the following Lemma:

Lemma 8.1.4. Aut $\left(\widehat{X}_{\sigma_{0}}\right)_{\text {Inv }}$ is connected.

Consequently, $\operatorname{Aut}(\sigma)_{\operatorname{Inv}} \subset p\left(\operatorname{Aut}\left(\widehat{X}_{\sigma_{0}}\right)_{\text {Inv }}\right) \subset \operatorname{Aut}\left(\widehat{X}_{\sigma}\right)^{0}$ and $\operatorname{Aut}(\sigma)_{\operatorname{Inv}} \subset \operatorname{Aut}(\sigma)^{0}$. 
Proof of Lemma 8.1.4. The same reasoning as in Lemma 4.9.9. Let $x_{1}, \ldots, x_{k}$ be the standard coordinates on $X_{\sigma_{0}}=\mathbf{A}^{k}$. Any automorphism $g \in \operatorname{Aut}\left(\widehat{X}_{\sigma_{0}}\right)_{\text {Inv }}$ is given by $\Gamma$-semiinvariant functions $g^{*}\left(x_{1}\right), \ldots, g^{*}\left(x_{k}\right)$ with the corresponding $\Gamma$-weights and such that $\operatorname{val}(v)\left(g^{*}\left(x_{i}\right)\right)=\operatorname{val}(v)\left(\left(x_{i}\right)\right)$, where $v \in \overline{\operatorname{Inv}}\left(\sigma_{0}\right) \cap N_{\sigma_{0}}$. There is a birational map $\alpha: \mathbf{A}^{1} \rightarrow G$ defined by

$$
\alpha(z):=\left(x_{1}, \ldots, x_{k}\right) \mapsto\left((1-z) g^{*}\left(x_{1}\right)+z x_{1}, \ldots,(1-z) g^{*}\left(x_{k}\right)+z x_{k}\right) .
$$

$\alpha(z)$ defines a $\Gamma$-equivarinat automorphism of $\widehat{X}_{\sigma_{0}}$ for all $z$ in the open subset $U$ of $\mathbf{A}^{1}$, where the linear parts of coordinates of $\alpha(z)$ are linearly independent. Note that for any integral vector $v \in \overline{\operatorname{Inv}}\left(\sigma_{0}\right)$ and $z \in U, \operatorname{val}(v)\left((1-z) g^{*}\left(x_{i}\right)+z x_{i}\right) \geq \operatorname{val}(v)\left(x_{i}\right)$. By Lemma 5.1.4, $\alpha(z)$ preserves val $(v)$.

Example 8.1.5. Let $X_{\sigma} \subset \mathbf{A}^{4}$ be a toric variety described by $x_{1} x_{2}=x_{3} x_{4}$. Then $\sigma=\left\langle v_{1}, v_{2}, v_{3}, v_{4}\right\rangle \subset N_{\sigma}^{\mathbf{Q}}$ is a cone over a square, where $N_{\sigma}^{\mathbf{Q}}:=\left\{\left(x_{1}, x_{2}, x_{3}, x_{4}\right) \mid x_{1}+x_{2}-x_{3}-x_{4}\right\} \subset \mathbf{Q}^{4}, v_{1}=(1,0,0,1), v_{2}=$ $(0,1,1,0), v_{3}=(1,0,1,0) v_{4}=(0,1,0,1)$. Then $\operatorname{Aut}(\sigma)=\mathbf{D}_{8}$ consists of all isometries of $\sigma$. Aut $(\sigma)_{\text {Inv }}$ consists of all isometries preserving diagonals: the reflections with respect to the diagonals and rotation through $\pi$. Consequently

$$
\Theta\left(X_{\sigma}, S, O_{\sigma}\right)=\operatorname{Aut}(\sigma) / \operatorname{Aut}(\sigma)_{\operatorname{Inv}} \simeq \mathbf{Z}_{2} .
$$

\subsection{Stratified toroidal varieties are orientible.}

Lemma 8.2.1. Let $\phi_{i}: X \rightarrow X_{\sigma}$ for $i=1,2$ be $\Gamma$-smooth morphisms of a $\Gamma$-stratified toroidal scheme $(X, S)$ to a $\Gamma$-stratified toric variety $\left(X_{\sigma}, S_{\sigma}\right)$ such that strata of $S$ are precisely the inverse images of strata of $S_{\sigma}$. Denote by $\Delta_{1}, \ldots, \Delta_{l}$ all subdivisions of $\sigma$ for which $\operatorname{Vert}\left(\Delta_{i}\right)=\operatorname{Vert}(\bar{\sigma})$. Let $v_{1}, \ldots, v_{r}$ be stable vectors such that $\operatorname{lin}\left(v_{1}, \ldots, v_{r}\right) \supseteq \operatorname{Inv}(\sigma)$. Denote by $\Delta_{l+1}, \ldots, \Delta_{l+r}$ the star subdivisions of $\sigma$ at $\left\langle v_{1}\right\rangle, \ldots,\left\langle v_{r}\right\rangle$. The following conditions are equivalent:

1. $\phi_{1}$ and $\phi_{2}$ determine the same orientation at a $K$-rational point $x \in s=\phi_{i}^{-1}$ (strat) $\left.\left.\sigma\right)\right)$.

2. There is an open neighborhood $U$ of $x$ such that $U_{j 1}:=U \times_{X_{\sigma}} X_{\Delta_{j}}$, defined via $\phi_{1}$, and $U_{j 2}:=$ $U \times_{X_{\sigma}} X_{\Delta_{j}}$, defined via $\phi_{2}$, are isomorphic over $U$ for any $j=1, \ldots, l+r$.

Proof. $(\Rightarrow)$ Suppose $\phi_{1}$ and $\phi_{2}$ determine the same orientation at $x$. By Proposition 7.6.1 the morphism $\widetilde{X}_{\Delta_{j}} \rightarrow \widetilde{X}_{\sigma}$ is $G^{\sigma}$-equivariant. Therefore by Lemma 4.13 .2 for any subdivision $\Delta_{j}$ of $\sigma$ there is an open neighborhood $U_{j}$ of $x$ such that $U_{j 1}^{\prime}:=U_{j} \times_{X_{\sigma}} X_{\Delta_{j}}$, defined via $\phi_{1}$, and $U_{j 2}^{\prime}:=U_{j} \times_{X_{\sigma}} X_{\Delta_{j}}$, defined via $\phi_{2}$, are isomorphic over $U_{j}$. It suffices to put $U=\bigcap U_{j}$.

$(\Leftarrow)$ Suppose $\phi_{1}$ and $\phi_{2}$ do not determine the same orientation at $x$. Then by Lemma 8.1 .2 we find an automorphism $\phi$ of $\sigma$ such that for the corresponding automorphism $\bar{\phi} \in \operatorname{Aut}\left(X_{\sigma}\right), \phi_{1}$ and $\bar{\phi} \phi_{2}$ determine the same orientation at $x$. By the above there is an open $U \ni x$ for which $U_{j, \phi_{1}}$ and $U_{j, \phi \phi_{2}}$ are isomorphic. But $\bar{\phi}$ does not preserve orientation so by 8.1.2, $\phi$ does not satisfy one of the conditions (1)-(2) of the lemma and does not lift to an automorphism of some $\Delta_{j} \neq \phi\left(\Delta_{j}\right)$. Consequently, $U_{j, \phi \phi_{2}} \simeq U_{j, \phi_{1}} \simeq U \times_{X_{\sigma}} X_{\Delta_{j}}$ is not isomorphic to $U_{j, \phi_{2}} \simeq U \times_{X_{\sigma}} X_{\phi\left(\Delta_{j}\right)}$ for any open neighborhood $U$ of $x$.

Lemma 8.2.2. Let $\left(X_{\Delta}, S_{\Omega}\right)$ be a $\Gamma$-stratified toric variety corresponding to an embedded semifan $\Omega \subset \Delta$. Let $\phi_{i}: X \rightarrow X_{\Delta}$ for $i=1,2$ be $\Gamma$-smooth morphisms of a $\Gamma$-stratified toroidal scheme $(X, S)$ to a $\Gamma$-stratified toric variety $\left(X_{\Delta}, S_{\Omega}\right)$ such that the strata of $S$ are precisely the inverse images of strata of $S_{\Omega}$.

For a cone $\omega \in \Omega$ denote by $\omega(\Delta)$ the subset $\{\tau \in \Delta \mid \omega(\tau)=\omega\}$ of $\Delta$. Let $\Delta_{1}, \ldots, \Delta_{l}$ be all subdivisions of $\omega$ satisfying condition (2) of Lemma 8.1.2. Let $\Delta_{l+1}, \ldots, \Delta_{l+r}$ denote the star subdivisions of $\omega$ at stable vectors $\left\langle v_{1}\right\rangle, \ldots,\left\langle v_{r}\right\rangle$ for which $\operatorname{lin}\left(v_{1}, \ldots, v_{r}\right) \supseteq \operatorname{Inv}(\sigma)$. Denote by $\Delta_{j}(\Delta)$ the subdivisions of $\omega(\Delta)$ induced by $\Delta_{j}$, i.e. for any $\delta=\omega(\delta) \oplus r(\delta) \in \omega(\Delta), \Delta_{j}(\Delta) \mid \delta=\Delta_{j} \oplus r(\delta)$. Let $x \in \phi_{i}^{-1}(\operatorname{strat}(\omega))$ be a K-rational point. The following conditions are equivalent:

1. $\phi_{1}^{*}(\mathcal{U}(\Delta, \Omega))$ and $\phi_{2}^{*}(\mathcal{U}(\Delta, \Omega))$ are compatible at $x$.

2. There is an open neighborhood $U$ of $x$ such that $U_{j 1}:=U \times_{X_{\omega(\Delta)}} X_{\Delta_{j}(\Delta)}$, defined via $\phi_{1}$, and $U_{j 2}$ : $U \times_{X_{\omega}(\Delta)} X_{\Delta_{j}(\Delta)}$, defined via $\phi_{2}$, are isomorphic over $U$ for any $j=1, \ldots, l+r$

Proof. Let $\phi_{i}(x) \in \delta_{i}$, where $\delta_{i} \in \Delta$ and $i=1,2$. Denote by $U_{i}$ the inverse image $\phi_{i}^{-1}\left(X_{\delta_{i}}\right)$. Since $x \in \phi_{i}^{-1}(\operatorname{strat}(\sigma))$, we have $\omega\left(\delta_{i}\right)=\omega$. The atlases $\phi_{i}^{*}\left(\mathcal{U}^{\text {can }}\left(X_{\Delta}, S_{\Delta}\right)\right)$ are compatible at $x$ if the morphisms $\pi_{\sigma}^{\sigma_{i}} \phi_{i}: U_{i} \rightarrow X_{\omega}$ determine the same orientation at $x$. This is equivalent by Lemma 8.2.1 to the fact that $U \times_{X_{\sigma}} X_{\Delta_{j}}$, defined via $\pi_{\sigma_{i}}^{\sigma} \phi_{i}$, are isomorphic for some $U$. The latter schemes are isomorphic to $U \times_{X_{\sigma_{i}}} X_{\Delta_{j}(\Delta) \mid \delta_{i}}=U \times_{X_{\omega(\Delta)}} X_{\Delta_{j}(\Delta)}$. 
JAROSŁAW WŁODARCZYK

Lemma 8.2.3. Let $\phi_{i}:(X, S) \rightarrow\left(X_{\Delta}, S_{\Omega}\right)$ for $i=1,2$ be $\Gamma$-smooth morphisms of a $\Gamma$-stratified toroidal scheme to a $\Gamma$-stratified toric variety $\left(X_{\Delta}, S_{\Omega}\right)$ such that the strata of $S$ are precisely the inverse images of strata of $S_{\Omega}$. Assume that a stratum $s \in S$ is a variety over $K$ and $\phi_{1}^{*}(\mathcal{U}(\Delta, \Omega))$ and $\phi_{2}^{*}(\mathcal{U}(\Delta, \Omega))$ are compatible at some point $x \in s$. Then they are compatible along $s$.

Proof. By Lemma 8.2.2, $\phi_{1}^{*}(\mathcal{U}(\Delta, \Omega))$ and $\phi_{2}^{*}(\mathcal{U}(\Delta, \Omega))$ are compatible at any $x \in s$ iff they are compatible at all points of $s \cap U_{x}$, where $U_{x}$ is an open neighborhood of $x$. Since $s$ is a variety the sets $s \cap U_{x}$ and $s \cap U_{y}$ intersect for any $x, y \in s$.

Lemma 8.2.4. Let $\phi_{1}, \phi_{2}: X \rightarrow X_{\sigma}$ be two $\Gamma$-smooth morphisms of a $\Gamma$-stratified toroidal variety $(X, S)$ into the $\Gamma$-stratified toric variety $X_{\sigma}$, such that the strata of $S$ are precisely the inverse images of strata of $S_{\sigma}$. Assume that $\phi_{1}, \phi_{2}$ determine the same orientation at a point $x \in \phi_{i}^{-1}(\operatorname{strat}(\sigma))$. Then $\phi_{i}^{*}\left(\mathcal{U}^{\text {can }}\left(X_{\sigma}, S_{\sigma}\right)\right)$ and $\phi_{2}^{*}\left(\mathcal{U}^{\text {can }}\left(X_{\sigma}, S_{\sigma}\right)\right)$ are compatible on $X$.

Proof. Let $\Delta_{1}, \ldots, \Delta_{l}, \ldots, \Delta_{l+r}$ be subdivisions of $\tau \leq \sigma$ as in Lemma 8.2.1. By Lemma 7.6.9, the subdivisions $\Delta_{1}, \ldots, \Delta_{l}$ can be extended to subdivisions $\Delta_{1}(\sigma), \ldots, \Delta_{l}(\sigma)$ of $\sigma$ for which $\operatorname{Vert}\left(\Delta_{i}\right)=\operatorname{Vert}(\bar{\sigma})$. By Lemma 7.4.4 $\operatorname{Inv}(\tau) \subset \operatorname{Inv}(\sigma)$ and we can extend the star subdivisions $\Delta_{l+1}, \ldots, \Delta_{l+r}$ to the star subdivisions $\Delta_{l+1}(\sigma), \ldots, \Delta_{l+r}(\sigma)$ of $\sigma$ at $v_{1}, \ldots, v_{r} \in \operatorname{Inv}(\tau) \subset \operatorname{Inv}(\sigma)$. By Lemma 4.13.2 there is an open neighborhood $U$ of $x$ for which $U_{j, \phi_{i}}=U \times_{X_{\sigma}} X_{\Delta_{j}}$ definded for $\phi_{1}$ and $\phi_{2}$ are pairwise isomorphic for any $j$. Let $\tau(\sigma)=\{\varrho \preceq \sigma \mid \omega(\varrho)=\tau\}$. Then there is an open subset $U^{\prime}=\phi_{1}^{-1}\left(X_{\tau(\sigma)}\right) \cap \phi_{2}^{-1}\left(X_{\tau(\sigma)}\right) \cap U$ intersecting $\phi_{i}^{-1}(\operatorname{strat}(\tau))$ such that $U^{\prime} \times_{X_{\sigma}} X_{\Delta_{j}}=U^{\prime} \times_{X_{\tau(\sigma)}} X_{\Delta_{j}(\sigma) \mid \tau(\sigma)}$ defined by the restrictions of $\phi_{i}$ are isomorphic. This implies by Lemma 8.2.2 that $\phi_{1}^{*}\left(\mathcal{U}^{\text {can }}\left(X_{\sigma}, S_{\sigma}\right)\right.$ and $\phi_{2}^{*}\left(\mathcal{U}^{\text {can }}\left(X_{\sigma}, S_{\sigma}\right)\right)$ are compatible at $x \in \operatorname{strat}(\tau) \cap U^{\prime}$ and consequently by Lemma 8.2 .3 they are compatible at all the points of $\phi_{i}^{-1}(\operatorname{strat}(\tau))$.

Lemma 8.2.5. The $\Gamma$-semicomplex $\Sigma$ associated to an oriented $\Gamma$-stratified toroidal variety $(X, S)$ is oriented.

Proof. For any $\sigma \leq \tau$, the inclusion morphism $\imath_{\sigma}^{\tau}: \sigma \rightarrow \tau$ identifies $\sigma$ with a face of $\tau$. Let $\bar{\pi}_{\tau}^{\sigma}: N_{\tau} \rightarrow N_{\sigma}$ denote a projection such that $\bar{\pi}_{\tau}^{\sigma} \circ \imath_{\sigma}^{\tau}=\operatorname{id}_{\mid \sigma}$. Let $\pi_{\tau}^{\sigma}: X_{\sigma} \rightarrow X_{\tau}$ denote the induced toric morphism. Consider faces $\sigma \leq \tau \leq \varrho$ of the semicomplex $\Sigma$. Let $\phi_{\sigma}: U_{\sigma} \rightarrow X_{\sigma}, \phi_{\tau}: U_{\tau} \rightarrow X_{\tau}, \phi_{\varrho}: U_{\varrho} \rightarrow X_{\varrho}$ denote any charts on $(X, S)$ corresponding to the cones $\sigma, \tau$ and $\varrho$. Since $\tau \leq \varrho$, the open subset $U_{\varrho}$ intersects $\tau$. For a generic point $x_{\tau}$ of $\operatorname{strat}(\tau) \cap U_{\varrho}$, the morphism $\phi_{\varrho}$ sends $x_{\tau}$ to the toric orbit $O_{\tau} \subset X_{\varrho}$. Since $(X, S)$ is oriented we find that $\pi_{\varrho}^{\tau} \phi_{\varrho}: U_{\varrho}^{\tau} \rightarrow X_{\tau}$ and $\phi_{\tau}: U_{\tau} \rightarrow X_{\tau}$ determine the same orientation at $x_{\tau}$. Analogously $\pi_{\varrho}^{\sigma} \phi_{\varrho}, \phi_{\sigma}$ and $\pi_{\tau}^{\sigma} \phi_{\tau}$ determine the same orientation for a generic point $x_{\sigma}$ of $\operatorname{strat}(\sigma)$. Applying Lemma 8.2.4 to $\pi_{\varrho}^{\tau} \phi_{\varrho}$ and $\phi_{\tau}$ we see that $\pi_{\tau}^{\sigma} \pi_{\varrho}^{\tau} \phi_{\varrho}$ and $\pi_{\tau}^{\sigma} \phi_{\tau}$ determine the same orientation for a generic point of strat $(\sigma)$. Finally $\pi_{\tau}^{\sigma} \pi_{\varrho}^{\tau} \phi_{\varrho}$ and $\pi_{\varrho}^{\sigma} \phi_{\varrho}$ determine the same orientation and thus so do $\pi_{\tau}^{\sigma} \pi_{\varrho}^{\tau}$ and $\pi_{\varrho}^{\sigma}$. Write $\imath_{\tau}^{\varrho} \imath_{\sigma}^{\tau}=\imath_{\sigma}^{\varrho} \alpha_{\sigma}$, where $\alpha_{\sigma} \in \operatorname{Aut}(\sigma)$. Then $\alpha_{\sigma} \pi_{\tau}^{\sigma} \pi_{\varrho}^{\tau} \imath_{\sigma}^{\varrho}=\alpha_{\sigma} \pi_{\tau}^{\sigma} \pi_{\varrho}^{\tau} \imath_{\tau}^{\varrho} \imath_{\sigma}^{\tau} \alpha_{\sigma}^{-1}=\mathrm{id}_{\mid \sigma}$. By Lemma 4.9.14 the toric morphisms induced by the projections $\alpha_{\sigma} \pi_{\tau}^{\sigma} \pi_{\varrho}^{\tau}$ and $\pi_{\varrho}^{\sigma}$ determine the same orientation. Thus the morphisms induced by $\alpha_{\sigma} \pi_{\tau}^{\sigma} \pi_{\varrho}^{\tau}$ and $\pi_{\tau}^{\sigma} \pi_{\varrho}^{\tau}$ determine the same orientation, which gives $\alpha_{\sigma} \in \operatorname{Aut}(\sigma)^{0}$.

Lemma 8.2.6. On any $\Gamma$-stratified toroidal variety $(X, S)$ there exists an atlas $\mathcal{U}$ such that $(X, S, \mathcal{U})$ is oriented.

Proof. We shall improve the charts from $\mathcal{U}$ and inclusion maps in the associated $\Gamma$-semicomplex $\Sigma$. For any $\sigma \in \Sigma$ fix a chart $\phi_{\sigma, a_{\sigma}}: U_{\sigma, a_{\sigma}} \rightarrow X_{\sigma}$. By Lemma 7.3.2 for any chart $\phi_{\sigma, a}: U_{\sigma, a} \rightarrow X_{\sigma}$ we can find $\psi_{\sigma, a}$ of $X_{\sigma}$ ) induced by an automorphism of $\sigma$ such that $\phi_{\sigma, a_{\sigma}}$ and

$$
\bar{\phi}_{\sigma, a}:=\psi_{\sigma, a} \circ \phi_{\sigma, a}
$$

determine the same orientation for some points of $\operatorname{strat}(\sigma) \cap U_{\sigma, a_{\sigma}} \cap U_{\sigma, a}$. By Lemma 8.2 .3 the above morphisms determine the same orientation for all points from $\operatorname{strat}(\sigma) \cap U_{\sigma, a_{\sigma}} \cap U_{\sigma, a}$.

Let $\sigma \leq \tau$. Denote by $\sigma^{\prime} \preceq \tau$ the face cone in $N_{\tau}$ such that $\underline{\sigma^{\prime}}=\sigma$. By Lemma 7.3 .2 find $\alpha_{\sigma} \in \operatorname{Aut}(\sigma)$ such that for the inclusion map $\overline{\imath_{\sigma^{\prime}}^{\tau}}:=\imath_{\sigma}^{\tau} \circ \alpha_{\sigma}$ any two charts $\phi_{\sigma, a_{\sigma}}: U_{\sigma, a_{\sigma}} \rightarrow X_{\sigma}, \pi_{\sigma^{\prime}}^{\sigma} \phi_{\tau, a_{\tau}}^{\sigma^{\prime}}: U_{\tau, a_{\tau}}^{\sigma^{\prime}} \rightarrow X_{\sigma}$ define the same orientation at some point of $x \in \operatorname{strat}(\sigma) \cap U_{\sigma, a_{\sigma}} \cap U_{\tau, a_{\tau}}^{\sigma^{\prime}}$. By Lemma 8.2.3 they determine the same orientation at all points of $\operatorname{strat}(\sigma) \cap U_{\sigma, a_{\sigma}} \cap U_{\tau, a_{\tau}}^{\sigma^{\prime}}$. We need to verify that for any two charts $\phi_{\sigma, a}$ and $\phi_{\tau, b}$, where $\sigma \leq \tau$, and any $\tau^{\prime} \preceq \tau$ such that $\omega\left(\tau^{\prime}\right)=\sigma$ the morphisms $\phi_{\sigma, a}$ and $\phi_{\tau, b}^{\tau^{\prime}}$ determine the same orientation for all $x \in \operatorname{strat}(\sigma) \cap U_{\sigma, a} \cap U_{\tau, b}^{\tau^{\prime}}$. By Lemma 8.2.3 it suffices to show that the above morphisms determine the same orientation for some $x \in \operatorname{strat}(\sigma) \cap U_{\sigma, a} \cap U_{\sigma, a_{\sigma}} \cap U_{\tau, b}^{\tau^{\prime}} \cap U_{\tau, a_{\tau}}^{\sigma}$. By the above $\phi_{\sigma, a}$ and $\phi_{\sigma, a_{\sigma}}$ determine 
the same orientation at all $x \in \operatorname{strat}(\sigma) \cap U_{\sigma, a} \cap U_{\sigma, a_{\sigma}}$. Also $\phi_{\tau, b}$ and $\phi_{\tau, a_{\tau}}$ determine the same orientation at all $x \in \operatorname{strat}(\tau) \cap U_{\tau, b} \cap U_{\tau, a_{\tau}}$. By Lemma 8.2.4, $\phi_{\tau, b}^{*}\left(\mathcal{U}^{\text {can }}\left(X_{\tau}, S_{\tau}\right)\right)$ and $\phi_{\tau, a_{\tau}}^{*}\left(\mathcal{U}^{\text {can }}\left(X_{\tau}, S_{\tau}\right)\right)$ are compatible on $U_{\tau, b} \cap U_{\tau, a_{\tau}}$. Hence $\phi_{\tau, b}^{\tau^{\prime}}$ and $\phi_{\tau, a_{\sigma}}^{\sigma^{\prime}}$ determine the same orientation at points $x \in \operatorname{strat}(\sigma) \cap U_{\tau, b}^{\tau^{\prime}} \cap U_{\tau, a_{\tau}}^{\sigma^{\prime}}$. Finally $\phi_{\sigma, a}$ and $\phi_{\tau, b}^{\tau^{\prime}}$ determine the same orientation for all $x \in \operatorname{strat}(\sigma) \cap U_{\sigma, a} \cap U_{\tau, b}^{\tau^{\prime}}$.

8.3. Resolution of singularities of toroidal varieties in arbitrary characteristic. We call a $\Gamma$ semicomplex $\Sigma$ regular if all cones $|\sigma|$, where $\sigma \in \Sigma$ are regular.

Proposition 8.3.1. For any oriented $\Gamma$-semicomplex $\Sigma$ there exists a sequence of stable vectors $v_{1}, \ldots, v_{m} \in$ $\operatorname{Stab}(\Sigma)$ such that the subdivision $\Delta:=\left\langle v_{k}\right\rangle \cdot \ldots \cdot\left\langle v_{1}\right\rangle \cdot \Sigma$ of $\Sigma$ is a regular $\Gamma$-semicomplex.

Proof. Let $\sigma_{1}, \ldots, \sigma_{k}$ denote all the faces of $\Sigma$. We shall construct by induction on $i$ the canonical subdivision $\Delta_{i}$ of $\Sigma$ such that the fan $\Delta_{i}^{\sigma_{j}}$ is regular for all $j \leq i$. Suppose $\Delta_{i}$ is already constructed. By [39] or 20] there exist centers $v_{1}^{i}, \ldots, v_{k_{i}}^{i}$ for the fan $\Delta_{i}^{\sigma_{i+1}}$ in $N_{\sigma_{i+1}}$ such that $\left\langle v_{k_{i}}^{i}\right\rangle \ldots \cdot\left\langle v_{1}^{i}\right\rangle \cdot \Delta_{i}^{\sigma_{i+1}}$ is regular. The subsequent centers $v_{j}^{i}$ of star subdivisions coul be chosen to be minimal internal vectors in indecomposable faces of $\left\langle v_{j-1}^{i}\right\rangle \cdot \ldots \cdot\left\langle v_{1}^{i}\right\rangle \cdot \Delta_{i}^{\sigma_{i+1}}$. Therefore by Lemma 5.3.16 all centers in the desingularization process are $\Sigma$-stable. Hence by Proposition 5.4.1, $\Delta_{i+1}:=\left\langle v_{k}^{i}\right\rangle \cdot \ldots \cdot\left\langle v_{1}^{i}\right\rangle \cdot \Delta_{i}$ is canonical. By construction $\Delta_{i+1}^{\sigma_{i+1}}$ is regular. Note that $\Delta_{i+1}^{\sigma_{j}}=\Delta_{i}^{\sigma_{j}}$ for $j<i+1$ since during the desingularization regular cones remain unaffected.

Theorem 8.3.2. For any $\Gamma$-stratified toroidal variety $(X, S)$ (respectively a $\Gamma$-toroidal variety $X)$ there exists a sequence of $\Gamma$-equivariant blow-ups at locally monomial valuations $\mathrm{bl}_{\nu_{k}} \circ \ldots \circ \mathrm{bl}_{\nu_{1}}(X)$ which is a resolution of singularities.

Proof. In the case of a nonstratified toroidal variety consider $X$ with stratification $\operatorname{Sing}^{\Gamma}(X)$. By Lemma 8.2.6 we can assume that the $\Gamma$-stratified toroidal variety is oriented. By Lemma 8.2.3 the associated semicomplex is oriented. By Proposition 8.3.1 and Proposition 5.4.1, $\mathrm{bl}_{\nu_{k}} \circ \ldots \circ \mathrm{bl}_{\nu_{1}}(X)$ is smooth.

\section{ORIENTATION OF TOROIDAL MODIFICATIONS}

9.1. Lifting group actions. Let $U$ be an affine variety. Let $\widehat{X}_{x}$ be a completion of a variety $X$ at its closed point $x$. Let $\Theta: Y \rightarrow X$ be a proper morphism and $\widehat{Y}_{x}:=\widehat{X}_{x} \times_{X} Y$.

Set

$$
\begin{gathered}
U \widehat{\times} \widehat{X}_{x}:=\operatorname{Spec}\left(\lim _{\leftarrow} K[U] \otimes \mathcal{O}_{x}^{n} / m_{X, x}\right), \\
U \widehat{\times} \widehat{Y}_{x}:=\left(U \widehat{\times} \widehat{X}_{x}\right) \times_{X} Y .
\end{gathered}
$$

Lemma 9.1.1. If $U, X$ are normal varieties then the scheme $U \widehat{X} \widehat{X}_{x}$ is normal. If $U, Y$ are normal varieties then the scheme $U \widehat{\times} \widehat{Y}_{x}$ is normal.

Proof. (1) $\pi_{X}: U \widehat{\times} \widehat{X}_{x} \rightarrow U \times \widehat{X}_{x}$ is étale at $(u, x)$ for $u \in U$ since it determines an isomorphism of the completions of the local rings at $(u, x) . U \times \widehat{X}_{x}$ is normal therefore $U \widehat{\times} \widehat{X}_{x}$ is normal at $(u, x) \in U \times\{x\}$. But all closed points of $U \widehat{\times} \widehat{X}_{x}$ are in $U \times\{x\} \subset U \widehat{\times} \widehat{X}_{x}$. The morphism $\pi$ is étale in a neighborhood of $U \widehat{\times} \widehat{X}_{x}$. Such a neighborhood is equal to $U \widehat{\times} \widehat{X}_{x}$ since its complement if nonempty would contain some closed points.

(2) $\pi_{Y}: U \widehat{\times} \widehat{Y}_{x} \rightarrow U \times \widehat{Y}_{x}$ is étale at $(u, y)$ for $u \in U$ and $y \in \theta^{-1}(x)$ since $\pi_{Y}$ is a pull-back of $\pi_{X}$. The rest of the reasoning is the same.

Definition 9.1.2. Let a proalgebraic group $G$ act on $\widehat{X}_{x}$. Let $\Phi: G \widehat{\times} \widehat{X}_{x} \rightarrow \widehat{X}_{x}$ be the action morphism and $\Psi: G \widehat{\times} \widehat{X}_{x} \rightarrow G \widehat{\times} \widehat{X}_{x}$ be the action automorphism (see Lemma 4.5.1). Let $\theta: U \rightarrow G$ be a morphism from a normal algebraic variety $U$ to $G$.

Then by the action morphism with respect to $U$ or simply action morphism we mean the induced morphism $\Phi_{U, \widehat{X}_{x}}: U \widehat{\times} \widehat{X}_{x} \rightarrow \widehat{X}_{x}$.

By the action automorphism with respect to $U$ or simply action automorphism we mean the induced $\operatorname{morphism} \Psi_{U, \widehat{X}_{x}}: U \widehat{\times} \widehat{X}_{x} \rightarrow U \widehat{\times} \widehat{X}_{x}$.

Let $\alpha: Y \rightarrow \widehat{X}_{x}$ be a $G^{K}$-equivariant birational morphism. By the lifting of the action morphism (resp. automorphism) we mean the morphism $\Phi_{U, Y}: U \widehat{\times} Y \rightarrow Y$ (resp. the automomophism $\left.\Psi_{U, Y}: U \widehat{\times} Y \rightarrow U \widehat{\times} Y\right)$ commuting with the induced morphism id $\widehat{\times} \alpha: U \widehat{\times} Y \rightarrow U \widehat{\times} \widehat{X}_{x}$ and with $\alpha$. 
Lemma 9.1.3. Let $G$ be a proalgebraic group acting on $\widehat{X}_{x}$. Let $U \rightarrow G$ be any morphism from a normal algebraic variety $U$. Let I be a G-invariant ideal on $\widehat{X}_{x}$. Let $\phi: Y \rightarrow \widehat{X}_{x}$ be the normalization of the blow-up at $I$. Then there is a lifting of the action automorphism (and action morphism) $\Psi_{U, \widehat{X}_{x}}: U \widehat{\times} \widehat{X}_{x} \rightarrow U \widehat{x}_{X}$ to $\Psi_{U, Y}: U \widehat{\times} Y \rightarrow U \widehat{\times} Y$.

Proof. Let $\Phi:=\Phi_{U, \widehat{X}_{x}}: U \widehat{\times} \widehat{X}_{x} \rightarrow \widehat{X}_{x}$ denote the action morphism. Set $\Psi:=\Psi_{U, \widehat{X}_{x}}$. By Lemma 4.5 .2 we know that $J=: p^{*}(I)=\Phi^{*}(I)=\Psi^{*} p^{*}(I)=\Psi^{*}(J)$ is preserved by the action automorphism. Therefore $J$ lifts to the blow-up of $U \widehat{\times} \widehat{X}_{x}$ at $J$ and to its normalization. Since $U \widehat{\times} \widehat{X}_{x} \rightarrow \widehat{X}_{x}$ is étale, the normalization of the blow-up of $U \widehat{\times} \widehat{X}_{x}$ at $J$ is isomorphic to $U \widehat{\times} Y$.

Proposition 9.1.4. Let $\sigma$ be a cone of maximal dimension in $N_{\sigma}$. Let $G$ be a connected proalgebraic group acting on $X:=\widehat{X}_{\sigma}$. Let $g: U \rightarrow G$ be a morphism from a normal algebraic variety $U$. Let $G^{K}$ act on $Y:=\widehat{X}_{\Sigma}$ such that $\psi: Y \rightarrow X$ is a $G^{K}$-equivariant proper birational morphism. Then there is a lifting of the action automorphism $\Psi_{U, X}: U \widehat{\times} X \rightarrow U \widehat{\times} X$ to $\Psi_{U, Y}: U \widehat{\times} Y \rightarrow U \widehat{\times} Y$.

Proof. Let $\Psi_{U, Y}: U \times Y-\rightarrow U \times Y$ be a birational map which is a lifting of $\Phi_{U, Y}$. By Lemmas 7.6 .8 and 9.1.3 we can find a factorization $Z \rightarrow Y \rightarrow X$ giving the diagram of proper morphisms

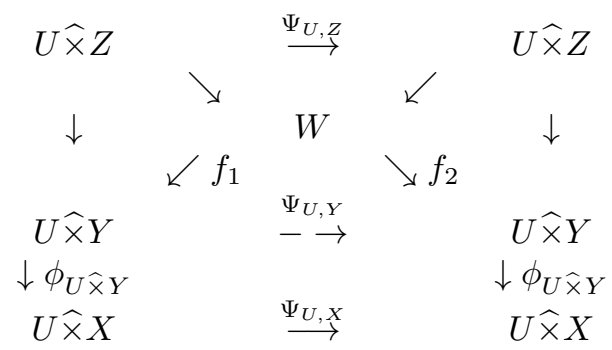

where $W$ is the irreducible component of the fiber product of $U \widehat{\times} Y \times_{U \widehat{\times} X} U \widehat{\times} Y$ which dominates $U \widehat{\times} Y$. (The fiber product is given by the morphisms $\phi_{U \hat{x} Y}$ and $\Psi_{U, X} \phi_{U \hat{x} Y}$.) Let $u \in U$ be a closed point. Take a pull-back of the above diagram via the closed embedding: $\{u\} \widehat{\times} X \rightarrow U \widehat{\times} X$

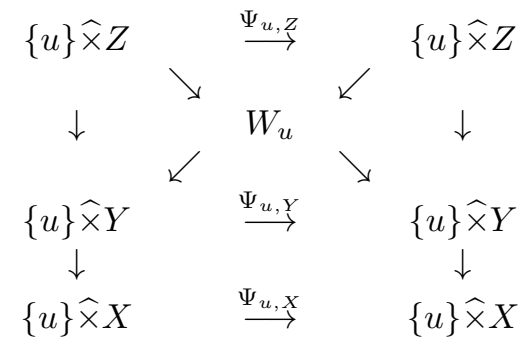

The birational map $\Psi_{u, Y}$ is the isomorphism defined by the action of $g(u) \in G$ on $Y$. $W_{u}$ consists of some components of the fiber product $\{u\} \widehat{\times} Y \times_{\{u\}} \widehat{\times} X\{u\} \widehat{\times} Y$. In particular it contains the irreducible component dominating $\{u\} \widehat{\times} Y$ which is isomorphic to $Y$. On the other hand $\{u\} \widehat{\times} Z \rightarrow W_{u}$ is a proper surjective morphism. Consequently, $W_{u}$ is irreducible and isomorphic to $Y$. By Lemma 9.1.1, $U \widehat{\times} Y$ is normal. Thus $f_{i}: W \rightarrow U \widehat{\times} Y$ is a proper birational morphism onto a normal scheme which is bijective on the set of closed ( $K$-rational) points. By the Zariski theorem $f_{i}$ is an isomorphism and $\Psi_{U, Y}=f_{1}^{-1} f_{2}$ is an action automorphism.

\subsection{Orientation of toroidal modifications.}

Proposition 9.2.1. Let $(X, S)$ be an oriented $\Gamma$-stratified toroidal variety with the associated $\Gamma$-semicomplex $\Sigma$. Let $f:(Y, R) \rightarrow(X, S)$ be the toroidal morphism associated to a canonical subdivision $\Delta$ of $\Sigma$. Then

1. The stratified toroidal variety $(Y, R)$ is oriented with charts from $\bigcup_{\sigma \in \Sigma} \phi_{\sigma}^{f *}\left(\mathcal{U}\left(\Delta^{\sigma}, \Delta_{\text {stab }}^{\sigma}\right)\right)$ induced by the charts $\phi_{\sigma}: U \rightarrow X_{\sigma}$ on $X$ and the associated oriented semicomplex $\Sigma_{R}=\Delta_{\text {stab }}$.

2. For any point $x$ in a stratum $s \in S$ every $\Gamma_{s}$-equivariant automorphism $\alpha$ of $\widehat{X}_{x}$ preserving strata and orientation lifts to a $\Gamma_{s}$-equivariant automorphism $\alpha^{\prime}$ of $Y \times_{X} \widehat{X}_{x}$ such that the atlases 
$\alpha^{\prime *} \hat{\phi}_{\sigma}^{f *}\left(\mathcal{U}\left(\Delta^{\sigma}, \Delta_{\text {stab }}^{\sigma}\right)\right)$ and $\hat{\phi}_{\sigma}^{f *}\left(\mathcal{U}\left(\Delta^{\sigma}, \Delta_{\text {stab }}^{\sigma}\right)\right)$ are compatible along $f^{-1}(x) \subset Y \times_{X} \widehat{X}_{x}$. Here $\hat{\phi}_{\sigma}^{f}$ : $Y \times_{X} \widehat{X}_{x} \rightarrow X_{\Delta^{\sigma}}$ denotes the morphism induced by $\phi_{\sigma}^{f}$.

3. The stable support of the oriented $\Gamma$-semicomplex $\Sigma_{R}=\Delta_{\text {stab }}$ is contained in the stable support of $\Sigma$.

Proof. $(\Leftarrow)$ Let $\phi_{\sigma, 1}: U_{\sigma, 1} \rightarrow X_{\sigma}$ and $\phi_{\tau, 2}: U_{\tau, 2} \rightarrow X_{\tau}$ be two charts on $X$, where $\sigma \leq \tau$. For (1) we have to show that $\phi_{\sigma, 1}^{f *}\left(\mathcal{U}\left(\Delta^{\sigma}, \Delta_{\text {stab }}^{\sigma}\right)\right)$ and $\phi_{\tau, 2}^{f *}\left(\mathcal{U}\left(\Delta^{\tau}, \Delta_{\text {stab }}^{\tau}\right)\right)$ are compatible on $f^{-1}\left(U_{\sigma, 1} \cap U_{\tau, 2}\right)$ along $f^{-1}(x)$ for any point $x \in \operatorname{strat}(\sigma) \cap\left(U_{\sigma, 1} \cap U_{\tau, 2}\right)$.

Let $\tau^{\prime} \preceq \tau$ denote a face of $\tau$ such that $\omega\left(\tau^{\prime}\right)=\sigma$ and $\phi_{\tau, 2}(x) \in O_{\tau^{\prime}}$. Then $\tau^{\prime}=\sigma \times r\left(\tau^{\prime}\right)$, where $r\left(\tau^{\prime}\right)$ denotes a regular cone and $\tau^{\prime} \backslash \sigma$ is disjoint from $\operatorname{Stab}(\Sigma)$. By Lemma 4.12.8, $\Delta^{\tau}\left|\tau^{\prime}=\Delta^{\tau}\right| \sigma \oplus r\left(\tau^{\prime}\right)=$ $\Delta^{\sigma} \times r\left(\tau^{\prime}\right)$. Also, $\Delta_{\text {stab }}^{\tau} \mid \tau^{\prime}=\Delta_{\text {stab }}^{\sigma} \times\{0\}$.

Denote by $\phi_{\tau, 2}^{\tau^{\prime}}: U_{\tau, 2}^{\tau^{\prime}} \rightarrow X_{\tau^{\prime}}$, the restriction of $\phi_{\tau, 2}$ and by $\phi_{\tau, 2}^{\tau^{\prime} f}$ its lifting. Then $\phi_{\tau, 2}^{\tau^{\prime} f *}\left(\mathcal{U}\left(\Delta^{\tau}\left|\tau^{\prime}, \Delta_{\text {stab }}^{\tau}\right| \tau^{\prime}\right)\right)$ is the restriction of $\phi_{\tau, 2}^{f *}\left(\mathcal{U}\left(\Delta^{\tau}, \Delta_{\text {stab }}^{\tau}\right)\right)$ to the open set $f^{-1}\left(U_{\tau, 2}^{\tau^{\prime}}\right)$ containing $f^{-1}(x)$. Set $U_{\sigma, 2}:=U_{\tau, 2}^{\tau^{\prime}}$, $\phi_{\sigma, 2}:=\pi_{\tau^{\prime}}^{\sigma} \phi_{\tau, 2}^{\tau^{\prime}}: U_{\sigma, 2} \rightarrow X_{\sigma}$, where $\pi_{\tau^{\prime}}^{\sigma}: X_{\tau}^{\prime} \rightarrow X_{\sigma}$ is defined by any projection which is identical on $\sigma \preceq \tau^{\prime}$. Let $\phi_{\sigma, 2}^{f}:=\pi_{\tau^{\prime}}^{\sigma f} \phi_{\tau, 2}^{\tau^{\prime} f}: f^{-1}\left(U_{\sigma, 2}\right) \rightarrow X_{\sigma}$ be the lifting of $\phi_{\sigma, 2}$. Then

$$
\phi_{\tau, 2}^{\tau^{\prime} f *}\left(\mathcal{U}\left(\Delta^{\tau}\left|\tau^{\prime}, \Delta_{\text {stab }}^{\tau}\right| \tau^{\prime}\right)\right)=\phi_{\tau, 2}^{\tau^{\prime} f *} \mathcal{U}\left(\Delta^{\sigma} \times r\left(\tau^{\prime}\right), \Delta_{\text {stab }}^{\sigma} \times\{0\}\right)
$$

is compatible along $f^{-1}(x)$ with

$$
\phi_{\tau, 2}^{\tau^{\prime} f *} \pi_{\tau^{\prime}}^{\sigma f *}\left(\mathcal{U}\left(\Delta^{\sigma}, \Delta_{\text {stab }}^{\sigma}\right)\right)=\phi_{\sigma, 2}^{f *}\left(\mathcal{U}\left(\Delta^{\sigma}, \Delta_{\text {stab }}^{\sigma}\right)\right) .
$$

It suffices to show that $\phi_{\sigma, 1}^{f *}\left(\mathcal{U}\left(\Delta^{\sigma}, \Delta_{\text {stab }}^{\sigma}\right)\right)$ and $\phi_{\sigma, 2}^{f *}\left(\mathcal{U}\left(\Delta^{\sigma}, \Delta_{\text {stab }}^{\sigma}\right)\right)$ are compatible along $f^{-1}(x)$.

Let $U \subset U_{\sigma, 1} \cap U_{\sigma, 2}$, be an open subset for which there exist étale extensions $\widetilde{\phi}_{i}: U \rightarrow X_{\widetilde{\sigma}}$ of $\phi_{\sigma, i}, i=1,2$. Assume that $\widetilde{\phi}_{i}(x)=O_{\widetilde{\sigma}}=O_{\sigma \times \operatorname{reg}(\sigma)}$, where $\operatorname{reg}(\sigma)$ is a regular cone of dimension $k=\operatorname{dim}(\operatorname{strat}(\sigma))$. Let $\widetilde{\phi}_{f i}$ be an étale extension of $\phi_{f i}$ which is a lifting of $\widetilde{\phi}_{i}$.

Consider the fiber squares

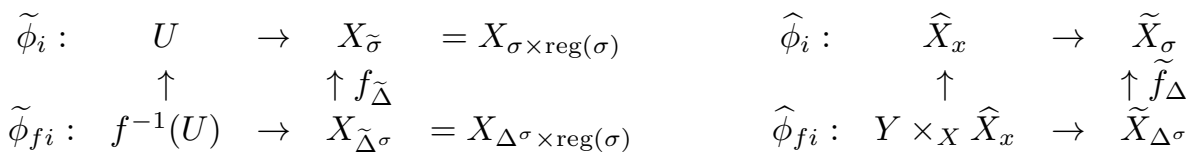

The morphism $\widetilde{f}_{\Delta}$ is a pull-back of the morphism $f_{\widetilde{\Delta}}$. Therefore $\widetilde{f}_{\Delta}^{-1}\left(O_{\widetilde{\sigma}}\right)$ is a $K$-subscheme of $\widetilde{X}_{\Delta^{\sigma}}$.

The automorphism $\widehat{\phi}:=\widehat{\phi}_{2} \widehat{\phi}_{1}^{-1}$ of $\widetilde{X}_{\sigma} \simeq \widehat{X}_{x}$ preserves strata and orientation. For the proof of conditions (1) and (2) we need to show that any such automorphism $\widehat{\phi}$ preserving strata and orientation lifts to the automorphism $\widehat{\phi}^{f}=\widehat{\phi}_{2}^{f}\left(\widehat{\phi}_{1}^{f}\right)^{-1}$ of $\widetilde{X}_{\Delta^{\sigma}} \simeq Y \times_{X} \widehat{X}_{x}$ such that $\widehat{\phi}^{f *}\left(\mathcal{U}\left(\Delta^{\sigma}, \Delta_{\text {stab }}^{\sigma}\right)\right.$ and $\mathcal{U}\left(\Delta^{\sigma}, \Delta_{\text {stab }}^{\sigma}\right)$ are compatible along $\widetilde{f}_{\Delta}^{-1}\left(O_{\widetilde{\sigma}_{\sigma}}\right)$.

By Lemma 7.3.1 there is an action morphism $\Phi: W \widehat{\times} \widetilde{X}_{\sigma} \rightarrow \widetilde{X}_{\sigma}$ such that

1. $\Phi_{e}:=\Phi_{\mid e \hat{x} \tilde{X}_{\sigma}}=\operatorname{id}_{X_{\sigma}}$.

2. There exists $g \in W$ such that $\Phi_{g}:=\Phi_{\mid g \widehat{\times} \tilde{X}_{\sigma}}=\widehat{\phi}$.

By Proposition 9.1.4, $\Phi$ can be lifted to $\Phi^{f}: W \widehat{\times} \widetilde{X}_{\Delta^{\sigma}} \rightarrow \widetilde{X}_{\Delta^{\sigma}}$ such that

3. $\Phi_{e}^{f}=\mathrm{id}_{\widetilde{X}_{\Delta^{\sigma}}}$.

4. $\Phi_{g}^{f}=\widehat{\phi}^{f}$.

Let $\Pi: W \widehat{\times} \widetilde{X}_{\sigma} \rightarrow \widetilde{X}_{\sigma}$ and $\Pi^{f}: W \widehat{\times} \widetilde{X}_{\Delta^{\sigma}} \rightarrow \widetilde{X}_{\Delta^{\sigma}}$ denote the natural projections.

Note that $\Pi^{-1}\left(O_{\tilde{\sigma}}\right)=\Phi^{-1}\left(O_{\tilde{\sigma}}\right)=W \widehat{\times} O_{\tilde{\sigma}} \simeq W \times O_{\widetilde{\sigma}} \simeq W$. The morphisms $\Pi^{f}$ and $\Phi^{f}$ are pull-backs of $\Pi$ and $\Phi$ induced by $\tilde{f}_{\Delta}$. Thus $\left(\Pi^{f}\right)^{-1}\left(f_{\widetilde{\Delta}}^{-1}\left(O_{\widetilde{\sigma}}\right)\right)=\left(\Phi^{f}\right)^{-1}\left(f_{\widetilde{\Delta}}^{-1}\left(O_{\tilde{\sigma}}\right)=W \widehat{\times} f_{\widetilde{\Delta}}^{-1}\left(O_{\tilde{\sigma}}\right) \simeq W \times f_{\widetilde{\Delta}}^{-1}\left(O_{\widetilde{\sigma}}\right)\right.$ is a $K$-subscheme of $W \widehat{\times} \widetilde{X}_{\Delta^{\sigma}}$.

For the sake of our considerations we shall enrich the stratification $R$ by adding to strata $r \in R$ their intersections with $f^{-1}(x)$. Set $\left.\bar{R}=\left\{r \backslash f^{-1}(x) \mid r \in R\right\}\right\} \cup\left\{r \cap f^{-1}(x) \mid r \in R\right\}$.

Strata of $R$ on $f^{-1}(U)$ correspond to the embedded semifan $\Delta_{\text {stab }}^{\sigma} \subset \Delta^{\sigma} \times \operatorname{reg}(\sigma)$. Strata of $\bar{R}$ correspond to the embedded semifan $\Omega \subset \Delta^{\sigma} \times \operatorname{reg}(\sigma)$, where $\Omega:=\Delta_{\text {stab }}^{\sigma} \cup\left\{\omega \times \operatorname{reg}(\sigma) \mid \omega \in \Delta_{\text {stab }}^{\sigma}\right.$, int $\left.(\omega) \subset \operatorname{int}(\sigma)\right\}$. 
For any $\operatorname{stratum}_{\operatorname{strat}_{Y}}(\omega) \in R, \omega \in \Delta_{\text {stab }}^{\sigma}$, dominating the $\operatorname{stratum}_{\operatorname{strat}}(\sigma)$ let strat $(\omega)$ denote the corresponding stratum on $\widetilde{X}_{\Delta^{\sigma}}$. Let $\widetilde{\omega}:=\omega \times \operatorname{reg}(\sigma) \in \Omega$. Then $\operatorname{strat}(\omega) \cap f_{\widetilde{\Delta}}^{-1}\left(O_{\widetilde{\sigma}}\right)=\operatorname{strat}(\widetilde{\omega}) \subset f_{\widetilde{\Delta}}^{-1}\left(O_{\widetilde{\sigma}}\right) \subset$ $\widetilde{X}_{\Delta^{\sigma}}$ is a $G^{\sigma}$-invariant stratum corresponding to $\widetilde{\omega} \in \Omega$. Since strat $(\widetilde{\omega})$ is an irreducible locally closed subset of $f_{\widetilde{\Delta}}^{-1}\left(O_{\widetilde{\sigma}}\right)$ it is an algebraic variety.

The natural isomorphism $\left(\Phi^{f}\right)^{-1}(\operatorname{strat}(\widetilde{\omega}))=\left(\Pi^{f}\right)^{-1}(\operatorname{strat}(\widetilde{\omega}))=W \widehat{\times} \operatorname{strat}(\widetilde{\omega}) \longrightarrow W \times \operatorname{strat}(\widetilde{\omega})$ is a pull-back of the isomorphism $W \widehat{\times} O_{\widetilde{\sigma}} \longrightarrow W \times O_{\widetilde{\sigma}}$ induced by $f_{\widetilde{\Delta}}^{-1}\left(O_{\widetilde{\sigma}}\right) \rightarrow O_{\widetilde{\sigma}}$.

Thus $W \widehat{\times} \operatorname{strat}(\widetilde{\omega})=W \times \operatorname{strat}(\widetilde{\omega})$ is an algebraic variety.

By definition we have $\Psi_{e}^{f}=\Pi_{e}^{f}=\operatorname{id}_{\mid \operatorname{strat}(\widetilde{\omega})}$. Hence by Lemma 4.10.5 applied to the natural projection of $W \widehat{\times} \operatorname{strat}(\widetilde{\omega})$ on $W$ the collections of charts $\Psi^{f *}\left(\mathcal{U}^{\text {can }}\left(\widetilde{X}_{\Delta^{\sigma}}, S_{\Omega}\right)\right)$ and $\Pi^{f *}\left(\mathcal{U}^{\text {can }}\left(\widetilde{X}_{\Delta^{\sigma}}, S_{\Omega}\right)\right)$ are compatible along $\{e\} \widehat{\times} \operatorname{strat}(\widetilde{\omega})$.

Hence by Lemma 8.2.3, $\Psi^{f *}\left(\mathcal{U}^{\text {can }}\left(\widetilde{X}_{\Delta^{\sigma}}, S_{\Omega}\right)\right)$ and $\Pi^{f *}\left(\mathcal{U}^{\text {can }}\left(\widetilde{X}_{\Delta^{\sigma}}, S_{\Omega}\right)\right)$ are compatible along $\{g\} \widehat{\times} \operatorname{strat}(\widetilde{\omega})$. Again by Lemma 4.10.5, $\Psi_{g}^{f *}\left(\mathcal{U}^{\operatorname{can}}\left(\widetilde{X}_{\Delta^{\sigma}}, S_{\Omega}\right)\right)=\widehat{\psi}^{f *}\left(\mathcal{U}^{\operatorname{can}}\left(\tilde{X}_{\Delta^{\sigma}}, S_{\Omega}\right)\right)$ and $\Pi_{g}^{f *}\left(\mathcal{U}^{\text {can }}\left(\tilde{X}_{\Delta^{\sigma}}, S_{\Omega}\right)\right)=\mathcal{U}^{\text {can }}\left(\widetilde{X}_{\Delta^{\sigma}}, S_{\Omega}\right)$ are compatible along strat $(\widetilde{\omega})$. The charts $\phi_{\omega^{\prime}}, \omega^{\prime} \geq \omega$, of $\mathcal{U}^{\text {can }}\left(\widetilde{X}_{\Delta^{\sigma}}, S_{\Delta_{\text {stab }}^{\sigma}}\right)$ are obtained by composing the charts $\phi_{\widetilde{\omega^{\prime}}}$ of $\mathcal{U}^{\operatorname{can}}\left(\widetilde{X}_{\Delta^{\sigma}}, S_{\Omega}\right)$ with the natural projection $X_{\widetilde{\omega}^{\prime}} \rightarrow X_{\omega^{\prime}}$. Thus the atlases $\mathcal{U}^{\text {can }}\left(\widetilde{X}_{\Delta^{\sigma}}, S_{\Delta_{\text {stab }}^{\sigma}}\right)$ and $\widehat{\psi}^{f *}\left(\mathcal{U}^{\text {can }}\left(\widetilde{X}_{\Delta^{\sigma}}, S_{\Delta_{\text {stab }}^{\sigma}}\right)\right.$ are compatible along $\operatorname{strat}(\omega) \cap f_{\widetilde{\Delta}}^{-1}\left(O_{\widetilde{\sigma}}\right)=\operatorname{strat}(\widetilde{\omega})$.

(3) By Lemma 8.2.5, $\Sigma_{R}=\Delta_{\text {stab }}$ is an oriented $\Gamma$-semicomplex and the notion of stability makes sense for

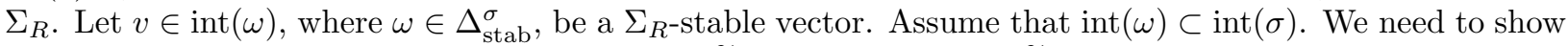
that for any $\tau \geq \sigma$, any automorphism $\alpha$ of $\operatorname{Aut}\left(\widetilde{X}_{\tau}\right)^{0} \operatorname{preserves} \operatorname{val}\left(v, \widetilde{X}_{\tau}\right)$. Find the cone $\omega^{\prime} \in \Delta^{\tau}$ such that $\omega^{\prime} \geq \omega$ and $\operatorname{int}\left(\omega^{\prime}\right) \subset \operatorname{int}(\tau)$. By the convexity of $\operatorname{stab}(\tau), \operatorname{int}\left(\omega^{\prime}\right)$ intersects $\operatorname{stab}(\tau)$ and $\omega^{\prime} \in \Delta^{\tau}$ stab.

Denote by $\pi: \widetilde{X}_{\Delta^{\tau}} \rightarrow X_{\Delta^{\tau}}$ the standard projection. By (2) $\alpha$ lifts to an automorphism $\alpha^{f}$ of $\tilde{X}_{\Delta^{\tau}}$ such that $\alpha^{f *} \pi^{*}\left(\mathcal{U}\left(\Delta^{\tau}, \Delta_{\text {stab }}^{\tau}\right)\right.$ and $\pi^{*}\left(\mathcal{U}\left(\Delta^{\tau}, \Delta_{\text {stab }}^{\tau}\right)\right.$ are compatible along $f_{\Delta}^{-1}\left(O_{\widetilde{\tau}}\right)$.

Let $p:=O_{\omega^{\prime} \times \operatorname{reg}(\tau)}=O_{\omega^{\prime}} \cap f_{\Delta}^{-1}\left(O_{\tilde{\tau}}\right) \subset \widetilde{X}_{\Delta^{\tau}}$. Set $Y:=\widetilde{X}_{\Delta^{\tau}}$. By Lemma 4.7.3, $\widehat{Y}_{p}=\widehat{X}_{\omega^{\prime} \times r e g(\tau)}=\widetilde{X}_{\omega^{\prime}}$.

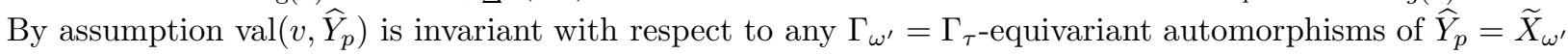
preserving orientation and strata. The automorphism $\alpha^{f}$ preserves $p$ and induces an automorphism $\widehat{\alpha}_{p}^{f}$ of $\widehat{Y}_{p}$. Denote by $\widehat{\pi}: \widehat{Y}_{p} \rightarrow X_{\omega^{\prime}}$ the morphism induced by $\pi$. Since the atlases $\alpha_{p}^{f *} \pi^{*}\left(\mathcal{U}\left(\Delta^{\tau}, \Delta_{\text {stab }}^{\tau}\right)\right.$ and $\pi^{*}\left(\mathcal{U}\left(\Delta^{\tau}, \Delta_{\text {stab }}^{\tau}\right)\right)$ are compatible the morphisms $\widehat{\pi} \widehat{\alpha}_{p}^{f}: \widehat{Y}_{p} \rightarrow X_{\omega^{\prime}}$ and $\widehat{\pi}$ determine the same orientation at $p$. Thus $\widehat{\alpha}_{p}^{f}$ preserves orientation at $p$ and $\widehat{\alpha}_{p *}^{f}\left(\operatorname{val}\left(v, Y_{p}\right)\right)=\operatorname{val}\left(v, Y_{p}\right)$. Then also $\alpha_{*}^{f}\left(\operatorname{val}\left(v, \operatorname{Spec}\left(\mathcal{O}_{Y, p}\right)\right)=\right.$ $\operatorname{val}\left(v, \operatorname{Spec}\left(\mathcal{O}_{Y, p}\right)\right.$. Consequently, $\alpha_{*}^{f}\left(\operatorname{val}\left(v, \widetilde{X}_{\Delta^{\tau}}\right)=\operatorname{val}\left(v, \widetilde{X}_{\Delta^{\tau}}\right)\right.$ and $\alpha_{*}\left(\operatorname{val}\left(v, \widetilde{X}_{\tau}\right)=\operatorname{val}\left(v, \widetilde{X}_{\Delta^{\tau}}\right)\right.$.

\section{The $\pi$-DESingularizATION LEMMA OF Morelli}

\subsection{Local projections of $\Gamma$-semicomplexes.}

Definition 10.1.1. Let $\Sigma$ be a simplicial $\Gamma$-semicomplex and $\Delta$ be its canonical subdivision. Let $\pi_{\sigma}: \sigma \rightarrow$ $\sigma^{\Gamma}$ be the projection defined by the quotient map $X_{\sigma} \rightarrow X_{\sigma} / \Gamma_{\sigma}=X_{\sigma^{\Gamma}}$, for any $\sigma \in \Sigma$. For any $\delta \in \Delta^{\sigma}$ set $\Gamma_{\delta}=\left(\Gamma_{\sigma}\right)_{\delta}=\left\{g \in \Gamma_{\sigma} \mid g x=x, x \in O_{\delta}\right\}$. By $\pi_{\delta}: \delta \rightarrow \delta^{\Gamma}$ denote the projection defined by the quotient map $X_{\delta} \rightarrow X_{\delta} / \Gamma_{\delta}=X_{\delta}$.

We say that $\Sigma$ is strictly $\pi$-convex if for any $\sigma \in \Sigma, \pi_{\sigma}(\sigma)=\sigma^{\Gamma}$ is strictly convex (contains no line). We call a semicomplex $\Sigma$ simplicial if all cones $|\sigma|, \sigma \in \Sigma$, are simplicial.

Lemma 10.1.2. 1. Let $\Sigma$ be a strictly convex $\Gamma$-semicomplex. Then for any $\tau \leq \sigma$, there exists an inclusion $\imath_{\tau}^{\sigma \Gamma}: \tau^{\Gamma} \hookrightarrow \sigma^{\Gamma}$ and the commutative diagram of inclusions:

$$
\begin{array}{cccc}
\imath_{\tau}^{\sigma}: & \tau & \hookrightarrow & \sigma \\
& \pi_{\tau} \downarrow & & \pi_{\sigma \downarrow} \downarrow \\
\imath_{\tau}^{\sigma \Gamma}: & \tau^{\Gamma} & \hookrightarrow & \sigma^{\Gamma}
\end{array}
$$

2. Let $\Delta$ be a canonical subdivision of $\Sigma$. Then for any $\delta \in \Delta_{\text {stab }}^{\sigma}$ we have the commutative diagram of inclusions

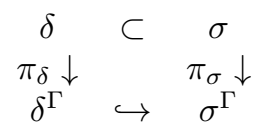


Proof. (1) Consider the commutative diagram

$$
\begin{aligned}
& X_{\left(\tau, N_{\sigma}\right)} \quad \simeq \quad X_{\tau} \times T \\
& \begin{array}{ccc}
X_{\left(\tau, N_{\sigma}\right)} / / \Gamma_{\tau} & \stackrel{i}{\simeq} & X_{\tau} / / \Gamma_{\tau} \times T \\
\downarrow & & \downarrow
\end{array} \\
& X_{\pi_{\sigma}\left(\tau, N_{\sigma}\right)}=X_{\left(\tau, N_{\sigma}\right)} / \Gamma_{\sigma} \stackrel{j}{\simeq} X_{\left(\tau, N_{\sigma}\right)} / / \Gamma_{\sigma} \times T / \Gamma_{\sigma} \simeq X_{\left(\tau^{\Gamma}, N_{\sigma}^{\Gamma}\right)}
\end{aligned}
$$

where $T$ is the relevant torus. Note that $\Gamma_{\tau}$ acts trivially on strat $(\tau) \subset X_{\sigma}$, hence in particular on the torus $T$ in $X_{\tau} \times T$. This gives the isomorphism $i$. Since $\Gamma_{\sigma} / \Gamma_{\tau}$ acts freely on $X_{\left(\tau, N_{\sigma}\right)} / / \Gamma_{\tau}=X_{\tau} / / \Gamma_{\sigma} \times T$ we get the isomorphism $j$. Consequently, $\left(\tau^{\Gamma}, N_{\sigma}^{\Gamma}\right) \simeq \pi_{\sigma}\left(\tau, N_{\sigma}\right) \subset \sigma^{\Gamma}$.

(2) Repeat the reasoning from (1) with appropriate inclusions.

10.2. Dependent and independent cones. In further considerations we shall assume $\Gamma=K^{*}$.

Lemma 10.2.1. Let $\Sigma$ be a simplicial strictly convex $K^{*}$-semicomplex. Let $\operatorname{Dep}(\Sigma):=\left\{\sigma \in \Sigma \mid \Gamma_{\sigma}=K^{*}\right\}$ and $\operatorname{Ind}(\Sigma):=\left\{\sigma \in \Sigma \mid \Gamma_{\sigma} \neq K^{*}\right\}$. Then

1. For $\sigma \in \operatorname{Ind}(\Sigma), \pi_{\sigma}$ is an immersion into the lattice $N_{\sigma}^{\Gamma}$ of the same dimension.

2. For $\sigma \in \operatorname{Dep}(\Sigma), \pi_{\sigma}$ is a submersion onto the lattice $N_{\sigma}^{\Gamma}$ such that $\operatorname{dim}\left(N_{\sigma}\right)=\operatorname{dim}\left(N_{\sigma}^{\Gamma}\right)+1$. Moreover there is a vector $v_{\sigma} \in N_{\sigma}$ determined by a $K^{*}$-action which spans the kernel of $\pi_{\sigma}$.

Proof. If $\sigma \in \operatorname{Ind}(\Sigma)$ then $\Gamma_{\sigma}$ is finite and there is an immersion $M_{\sigma}^{\Gamma} \rightarrow M_{\sigma}$, of lattices of the same dimension, where $M^{\Gamma}$ is a lattice of $\Gamma$-invariant characters. The dual morphism $N_{\sigma} \rightarrow N_{\sigma}^{\Gamma}$ is also an immersion of lattices of the same dimension. If $\sigma \in \operatorname{Dep}(\Sigma)$ then $\Gamma_{\sigma}=K^{*}$ is a subtorus corresponding to the sublattice $N:=\left(\mathbf{Q} \cdot v_{\sigma}\right) \cap N_{\sigma}$ of $N_{\sigma}$. The projection $\pi_{\sigma}$ is defined by the quotient map $N_{\sigma} \rightarrow N_{\sigma} / N \simeq N_{\sigma}^{\Gamma}$.

Lemma 10.2.2. Let $\Sigma$ be a simplicial strictly convex $K^{*}$-semicomplex. Then for any faces $\tau \leq \sigma$ in $\operatorname{Dep}(\Sigma)$, $\imath_{\tau}^{\sigma}\left(v_{\tau}\right)=v_{\sigma}$.

Proof. The induced toric morphism $X_{\tau} \rightarrow X_{\sigma}$ is $K^{*}$-equivariant.

Definition 10.2.3. (see Morelli [49]) Let $\Delta$ be a canonical subdivision of a $K^{*}$-semicomplex $\Sigma$. Let $\sigma \in \Sigma$.

1. A cone $\delta \in \Delta^{\sigma}$ is called independent if the restriction of $\pi_{\sigma}$ to $\delta$ is a lattice immersion. Otherwise $\delta \in \Delta^{\sigma}$ is called dependent.

2. A minimal dependent face of $\Delta^{\sigma}$ is called a circuit.

3. We call an independent face $\tau$ up-definite (respectively down-definite) with respect to a dependent face $\delta$ if there exists a nonzero functional $F$ on $\delta \subset N_{\sigma}^{\mathbf{Q}}$ such that $F\left(v_{\sigma}\right)>0$ (respectively $F\left(v_{\sigma}\right)<0$ ), and $\tau=\{v \in \sigma \mid F(v)=0\}$.

Lemma 10.2.4. Each dependent cone $\delta=\left\langle v_{1}, \ldots, v_{k}\right\rangle \in \Delta^{\sigma}$ defines a unique linear dependence relation

$$
r_{1} \pi_{\sigma}\left(v_{1}\right)+\ldots+r_{k} \pi_{\sigma}\left(v_{k}\right)=0
$$

This relation is determined up to proportionality.

Proof. $\pi_{\sigma}(\delta)$ is $k-1$ - dimensional cone spanned by $k$ rays.

Definition 10.2.5. $\quad 1$. The relation $\left(^{*}\right)$ is positively normalized if the rays $\left\langle v_{i}\right\rangle$ for which $r_{i}>0$ form an up-definite face

2. The rays for which $r_{i}>0$ (in a positively normalized relation) are called positive, the rays for which $r_{i}<0$ are called negative, the rays for which $r_{i}=0$ are called null rays. The face $\tau$ of a cone $\delta$ is called codefinite if it contains only positive or only negative rays.

3 . For any cone $\delta$ we denote by $\delta_{-}$(respectively $\delta_{+}$) the fan consisting of all faces of $\delta$ which are downdefinite (respectively up-definite). By $\delta^{-}$(respectively $\delta^{+}$) we denote the face of $\delta$ spanned by all negative rays and null rays (respectively by all positive rays and null rays).

Lemma 10.2.6. The relation $\left(^{*}\right)$ is positively normalized iff there exists $\alpha>0$, such that

$$
r_{1} v_{1}+\ldots+r_{k} v_{k}=\alpha v_{\delta}
$$


Proof. Let $F$ be a nonnegative functional on $\delta$ which is 0 exactly on $\left\langle v_{i} \mid r_{i}>0\right\rangle$. Then $\left\langle v_{i} \mid r_{i}>0\right\rangle$ is up-definite iff $F\left(v_{\delta}\right)>0$. The latter is equivalent to $\alpha>0$.

Lemma 10.2.7. Let $\tau=\left\langle v_{1}, \ldots, \check{v}_{i}, \ldots, v_{k}\right\rangle$ be a face of $\delta$ of codimension 1. Then $\tau$ is up-definite iff $r_{i}>0$.

Proof. Let $F$ be a nonnegative functional on $\delta$ which is 0 exactly on $\tau$. Then by Lemma 10.2.6, $r_{i}>0$ iff $F\left(v_{\delta}\right)>0$. The latter means that $\tau$ is up-definite.

Lemma 10.2.8. If $\tau \preceq \delta$ is up-definite (respectively down-definite) then any face $\tau^{\prime} \preceq \tau$ is up-definite (respectively down-definite) with respect to $\delta$.

Proof. Let $F$ be a nonnegative functional on $\delta$ which is 0 exactly on $\tau$ and such that $F\left(v_{\delta}\right)>0$. Let $F^{\prime}$ be a nonnegative functional on $\tau$ which is 0 exactly on $\tau^{\prime}$. Then $n F+F^{\prime}$, where $n>>0$, is a nonnegative functional on $\delta$ which is 0 exactly on $\tau^{\prime}$ and $\left(n F+F^{\prime}\right)\left(v_{\delta}\right)>0$.

Lemma 10.2.9. The set $\delta_{+}$(respectively $\delta_{-}$) is a subfan of $\bar{\delta}$ with maximal faces of the form $\left\langle v_{1}, \ldots, \check{v}_{i}, \ldots, v_{k}\right\rangle$, where $r_{i}>0$ (respectively $\left.r_{i}<0\right)$. In particular, each boundary face is up-definite or down-definite. The projection $\pi_{\delta}$ maps $\delta_{+}$(respectively $\delta_{-}$) onto the subdivision $\pi_{\delta}\left(\delta_{+}\right)$(respectively $\pi_{\delta}\left(\delta_{-}\right)$of $\pi_{\delta}(\delta)$. Moreover the restriction of $\pi$ to any boundary face is a linear isomorphism.

Proof. Let $v$ be a vector in $\left|\delta^{\Gamma}\right|$. Then either the line $\pi^{-1}(v)=\{v\}+\operatorname{lin}\left(v_{\delta}\right)$ intersects the relative interiors of exactly two boundary faces at one point each and $\pi^{-1}(v) \cap \delta$ is an interval or $\pi^{-1}(v)$ intersects the relative interior of one boundary face at a point and $\pi^{-1}(v) \cap \delta$ is the point. In the first case one of the faces is up-definite and one is down-definite. In the second case let $\tau$ be the boundary face containing $p=\pi^{-1}(v) \cap \delta$. Let $F$ be a nonnegative functional on $\delta^{\Gamma}$ which is zero exactly on $\tau$. Let $F^{\prime}$ be any functional on $N_{\delta}^{\mathbf{Q}}$ which equals 0 on $\tau$ and such that $F^{\prime}\left(v_{\delta}\right) \geq 0$. Then $(n F \circ \pi) \pm F^{\prime}$, where $n>>0$, is nonnegative on $\delta$ and equals 0 exactly on $\tau$. Moreover $\left(n F \circ \pi+F^{\prime}\right)\left(v_{\delta}\right)>0$ and $\left(n F \circ \pi-F^{\prime}\right)\left(v_{\delta}\right)<0$. Hence $\tau$ is up-definite and down-definite.

Lemma 10.2.10. Let $\delta \in \Delta^{\sigma}$ be a dependent cone. Set $\delta^{+}:=\left\langle v_{i} \mid r_{i}>0\right\rangle$. The ideal $I_{\bar{O}_{\delta^{+}}}$of the closure of $O_{\delta^{+}}$is generated by the set of all characters with positive $K^{*}$-weights.

Proof. Let $x^{m}, m \in \delta^{\vee} \cap M_{\delta}$ be the character with the positive weight. Then $\left(m, v_{\delta}\right)>O$. By Lemma 10.2.6, there is $r_{i}>0$ in a positively normalized relation such that $\left(m, v_{i}\right)>0$. Then $x^{m}$ is zero on the divisor corresponding to $v_{i}$ and on $O_{\delta^{+}}$. On the other hand for any $r_{i}>0$ we can find a functional $m_{i}$ such that $\left(m_{i}, v_{i}\right)>0,\left(m_{i}, v_{\delta}\right)>O,\left(m_{i}, v_{j}\right)=0$, where $j \neq i$. Then $\bar{O}_{\delta^{+}}$is the set of the common zeros of $x^{m_{i}}$, $r_{i}>0$.

We associate with a cone $\delta \in \Delta^{\sigma}$ and an integral vector $v \in \delta^{\Gamma}$ a vector $\operatorname{Mid}(v, \delta) \in \delta$ (44]. If $\delta$ is independent face, then $\operatorname{Mid}(v, \delta)$ is defined to be the primitive vector of the ray $\mathbf{Q}_{\geq 0} \cdot \pi_{\sigma}^{-1}(v) \subset \delta$. If $\delta$ is dependent let $\pi_{\delta_{-}}=\pi_{\sigma \mid \delta_{-}}$and $\pi_{\delta_{+}}=\pi_{\sigma \mid \delta_{+}}$be the restrictions of $\pi_{\sigma}$ to $\delta_{-}$and $\delta_{+}$. Then $\operatorname{Mid}(v, \delta)$ is the primitive vector of the ray spanned by $\pi_{\delta_{-}}^{-1}(v)+\pi_{\delta_{+}}^{-1}(v) \in \delta$.

Remark. By Lemma 10.1.2 the local projections $\pi_{\sigma}: N_{\sigma} \rightarrow N_{\sigma}^{\Gamma}$ commute with subdivisions and face restrictions therefore the above notions are coherent.

10.3. Stable vectors on simplicial $K^{*}$-semicomplexes. In the section $\Sigma$ denotes an oriented strictly convex $K^{*}$-semicomplex.

Lemma 10.3.1. Let $\sigma$ be a semicone in $\Sigma$. Then $G_{\sigma}$ acts on

$$
\widetilde{X}_{\sigma^{\Gamma}}:=\widetilde{X}_{\sigma} / \Gamma_{\sigma}
$$

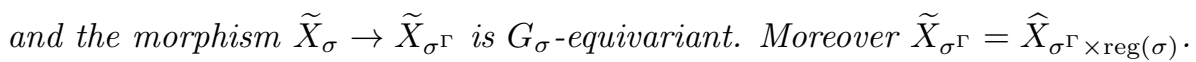

Proof. Follows from the fact that the action of $G^{\sigma}$ commutes with $\Gamma_{\sigma}$.

Lemma 10.3.2. Let $\sigma$ be a dependent cone in $\Sigma$. Then

1. $\tilde{X}_{\sigma_{-}}:=X_{\sigma_{-}} \times_{X_{\sigma}} \tilde{X}_{\sigma} \subset \tilde{X}_{\sigma}$ is $G_{\sigma}$-invariant.

2. $\tilde{X}_{\pi_{\sigma_{-}}\left(\sigma_{-}\right)}:=X_{\pi_{\sigma_{-}}\left(\sigma_{-}\right)} \times_{X_{\pi(\sigma)}} \widetilde{X}_{\pi(\sigma)} \rightarrow \widetilde{X}_{\pi(\sigma)}$ is proper $G_{\sigma}$-equivariant. 
Proof (1) By Lemma 10.2.9, $\sigma_{-}=\bar{\sigma} \backslash \operatorname{Star}\left(\sigma^{+}, \bar{\sigma}\right)$. By Lemma 10.2.6, the ideal of $\bar{O}_{\sigma^{+}}=\widetilde{X}_{\sigma} \backslash \widetilde{X}_{\sigma_{-}}$is generated by all semi-invariant functions with positive weights (see also [73], Example 2).

(2) Follows from Proposition 7.6 .1 since $\operatorname{Vert}\left(\pi_{\sigma_{-}}\left(\sigma_{-}\right)\right)=\operatorname{Vert}(\pi(\sigma))$

Lemma 10.3.3. Let $\sigma$ be a cone of the maximal dimension. Let $F$ denote the functional on $\tau:=\sigma^{\vee}$ defined by some integral vector $v \in N_{\sigma}$ which is not in $\sigma$. For any integral $k$ set $\tau_{k}:=\{v \in \tau \mid F(v)=k\}$. Then there are a finite number of vectors $w_{k 1}, \ldots, w_{k l_{k}} \in \tau_{k}$ such that

$$
\tau_{k}=\bigcup_{i=1, \ldots, l_{k}}\left(w_{k i}+\tau_{0}\right) .
$$

Proof. We can replace $\tau$ by a regular cone $\tau_{0}$ by considering the epimorphism $p: \tau_{0} \rightarrow \tau$ mapping generators to generators. Then $F$ defines a functional on $\tau_{0}$. Let $\tau_{0 k}:=\left\{v \in \tau_{0} \mid F(v)=k\right\}$. Let $x_{1}, \ldots, x_{m}$ define the standard coordinates on $\tau \simeq \mathbf{Z}_{\geq 0}^{m}$. Without loss of generality we can write $F=n_{1} x_{1}+\ldots+n_{l} x_{l}-$ $n_{l+1} x_{l+1}-\ldots-n_{r} x_{r}$, where $l<r \leq m$ and all $n_{i}>0$. Then $\tau_{0 k}=\left(\tau_{0 k} \cap\left(\mathbf{Z}_{\geq 0}^{r} \times\{0\}\right)\right)+\left(\{0\} \times \mathbf{Z}^{m-r}\right)$, where $\{0\} \times \mathbf{Z}^{m-r} \subset \tau_{00}$.

By a k-minimal vector we shall mean a vector $v \in \tau_{0 k} \cap\left(\mathbf{Z}_{\geq 0}^{r} \times\{0\}\right)$ such that there is no $w \in \tau_{00} \cap\left(\mathbf{Z}_{\geq 0}^{r} \times\right.$ $\{0\})$ for which $v-w \in \tau_{0 k}$. It suffices to show that the number of $k$-minimal vectors is finite. Suppose $x_{i_{0}}(v) \geq\left(n_{1}+\ldots+n_{l}\right)\left(n_{l+1}+\ldots+n_{r}\right)+|k|$ for some $i_{0} \leq l$ and let $x_{j_{0}}:=\max \left\{x_{i}(v) \mid l+1 \leq i \leq r\right\}$. Then $x_{j_{0}} \geq \frac{n_{1} x_{1}+\ldots+n_{l} x_{l}}{n_{1}+\ldots+n_{l}}=\frac{n_{l+1} x_{l+1}+\ldots+n_{r} x_{r}+k}{n_{l+1}+\ldots+n_{r}} \geq\left(n_{1}+\ldots+n_{l}\right)\left(n_{l+1}+\ldots+n_{r}\right) /\left(n_{l+1}+\ldots+n_{r}\right)=n_{1}+\ldots+n_{l}$

Then $w:=\left(0, \ldots, n_{j_{0}}, \ldots, 0, \ldots, n_{i_{0}} \ldots 0\right) \in \tau_{00}$, where the $i_{0}$-th coordinate is $n_{j_{0}}$ and the $j_{0}$-th coordinate is $n_{i_{0}}$. Thus $v-w \in \tau_{0 k}$ and $v$ is not $k$-minimal. Consequently, all minimal $k$-vectors satisfy $\max \left\{x_{i}(v) \mid 1 \leq i \leq r\right\}<\left(n_{1}+\ldots+n_{l}\right)\left(n_{l+1}+\ldots+n_{r}\right)+|k|$.

As a corollary we get

Lemma 10.3.4. Let $K\left[\widetilde{X}_{\sigma}\right]=\bigoplus K\left[\widetilde{X}_{\sigma}\right]^{k}$ be the decomposition according to weights with respect to the $\Gamma_{\sigma}=K^{*}$-action. Then $K\left[\widetilde{X}_{\sigma}\right]^{k}$ is a finitely generated $K\left[\widetilde{X}_{\sigma}\right]^{0}$-module.

Lemma 10.3.5. Let $\sigma$ be a dependent cone in $\Sigma$. Denote by $j_{\pi_{\sigma_{-}}}: X_{\sigma_{-}} \rightarrow X_{\sigma_{-}} / \Gamma_{\sigma}$ and $\widetilde{j}_{\pi_{\sigma_{-}}}: \widetilde{X}_{\sigma_{-}} \rightarrow \widetilde{X}_{\sigma_{-}} / \Gamma_{\sigma}$ the quotient morphisms. Then

1. $\tilde{X}_{\sigma_{-}} / \Gamma_{\sigma} \simeq \widetilde{X}_{\pi_{\sigma_{-}}\left(\sigma_{-}\right)}$.

2. $\tilde{j}_{\pi_{\sigma_{-}}}: \tilde{X}_{\sigma_{-}} \rightarrow \widetilde{X}_{\pi_{\sigma_{-}}\left(\sigma_{-}\right)}$is $G_{\sigma^{-}}$equivariant.

3. For any $v \in \pi\left(\sigma_{-}\right), j_{\pi}^{*}\left(\operatorname{val}\left(v, X_{\pi_{\sigma_{-}}\left(\sigma_{-}\right)}\right)\right)=\operatorname{val}\left(\pi_{\sigma_{-}}^{-1}(v), X_{\sigma_{-}}\right)$.

4. If $\operatorname{val}\left(v, \widetilde{X}_{\pi_{\sigma_{-}}\left(\sigma_{-}\right)}\right)$is $G^{\sigma}$ invariant then $\tilde{j}_{\pi_{\sigma_{-}}}^{*}\left(\operatorname{val}\left(v, \widetilde{X}_{\pi_{\sigma_{-}}\left(\sigma_{-}\right)}\right)\right)=\operatorname{val}\left(\pi_{\sigma_{-}}^{-1}(v), \widetilde{X}_{\sigma_{-}}\right)$is $G^{\sigma}$-invariant.

Proof. (1) For $\tau \in \sigma_{-}$set $\widetilde{X}_{\tau}:=X_{\left(\tau, N_{\sigma}\right)} \times_{X_{\sigma}} \widetilde{X}_{\sigma}$,

$\widetilde{X}_{\pi_{\sigma_{-}}\left(\tau, N_{\sigma}\right)}:=X_{\pi_{\sigma_{-}}(\tau)} \times_{X_{\pi(\sigma)}} \tilde{X}_{\pi(\sigma)}$.

Then $K\left[\widetilde{X}_{\tau}\right]=K\left[X_{\left(\tau, N_{\sigma}\right)}\right] \otimes_{K\left[\tilde{X}_{\sigma}\right]} K\left[\widetilde{X}_{\sigma}\right]$.

The elements of this ring are finite sums $\sum \chi_{i} f_{i}$, where $f_{i} \in K\left[\widetilde{X}_{\sigma}\right]$ and $\chi_{i} \in \tau^{\vee}$ is a character.Set

$R_{1}:=K\left[\widetilde{X}_{\tau} / \Gamma_{\sigma}\right]=K\left[\tilde{X}_{\tau}^{\Gamma_{\sigma}}\right]=\left(K\left[X_{\tau}\right] \otimes_{K\left[X_{\sigma}\right]} K\left[\tilde{X}_{\sigma}\right]\right)^{\Gamma_{\sigma}}$,

$R_{2}:=K\left[\widetilde{X}_{\pi_{\sigma_{-}}(\tau)}\right]=K\left[X_{\pi_{\sigma_{-}}(\tau)}\right] \otimes_{K\left[X_{\pi(\sigma)}\right]} K\left[\widetilde{X}_{\pi(\sigma)}\right] \subset R_{1}$.

$R_{2}$ consists of elements $\sum \chi_{i} f_{i}$, where $\chi_{i} \in \tau^{\vee}$ and $f_{i} \in K\left[\widetilde{X}_{\sigma}\right]$ have weight $0 . \quad R_{1}$ consists of elements $\sum \chi_{i} f_{i}$, where $\chi_{i} \in \tau^{\vee}$ and $f_{i} \in K\left[\widetilde{X}_{\sigma}\right]$ have opposite weights. By Lemma 10.3 .4 there is a finite number of characters $\chi_{i j} \in \sigma^{\vee}$ such that $f_{i}=\sum \chi_{i j} f_{i j}$, where $f_{i j}$ have weight zero. Finally, $\sum \chi_{i} f_{i}=\sum_{i} \chi_{i}\left(\sum_{j} \chi_{i j} f_{i j}\right)=\sum_{i} \sum_{j}\left(\chi_{i} \chi_{i j}\right) f_{i j} \in R_{2}$.

(2) Follows from (1).

(3) By Lemma 10.2 .9 the projection $\pi:=\pi_{\sigma_{-}}$maps cones of $\sigma_{-}$isomorphically onto cones of $\pi\left(\sigma_{-}\right)$.

By [39], Ch. I Th. 9, the sheaf of ideals $\mathcal{I}:=\mathcal{I}_{\mathrm{val}(\pi(v)), d}$ on $X_{\pi_{\sigma_{-}}\left(\sigma_{-}\right)}$corresponds to the strictly convex piecevise linear function $\operatorname{ord}(\mathcal{I})$. By definition and Lemma 5.2.1, $\operatorname{ord}(\mathcal{I})$ equals 0 on all cones not containing $v$. Let $\tau \in \sigma_{-}$be a cone containing $v$. By the proof of Lemma 5.2.8, if $d$ is sufficiently divisible then for any face $\delta$ of $\tau$ such that $\delta$ does not contain $v$, there is an integral vector $m_{\pi(\delta), \pi(\tau)} \in \pi(\tau)^{\vee}$ for which $\left(m_{\pi(\delta), \pi(\tau)}, \pi(v)\right)=d, m_{\pi(\delta), \pi(\tau)}$ is 0 on $\pi(\delta)$ and $\operatorname{ord}(\mathcal{I})$ equals $m_{\pi(\delta), \pi(\tau)}$ on $\pi(\delta+\langle v\rangle)$. 
JAROSŁAW WŁODARCZYK

In particular $\mathcal{I}$ is generated on each cone $X_{\pi(\tau)}$ by $m_{\pi(\delta), \pi(\tau)}$, where of $\delta$ is a face of $\tau$ that does not contain $v$. Let $m_{\delta}=m_{\pi(\delta)} \circ \pi \in \tau^{\vee}$ be the induced functional on $M_{\tau}$. Thus the ideal $\pi^{*}(\mathcal{I})$ is generated on each cone $X_{\tau}$, where $\tau \in \sigma_{-}$contains $v$, by $m_{\delta, \tau}$, where $\delta$ doesn not contain $v$. Then $\left(m_{\delta, \tau}, v\right)=d, m_{\delta}$ is 0 on $\delta$ and $\operatorname{ord}\left(\pi^{*}(\mathcal{I})\right)$ equals $m_{\delta, \tau}$ on $\delta+\langle v\rangle$. This gives by the proof of Lemma 5.2.8, $\pi^{*}\left(\mathcal{I}_{\operatorname{val}(\pi(v)), d}\right)=\mathcal{I}_{\mathrm{val}(v), d}$.

(4) Follows from (2) and (3).

Lemma 10.3.6. Let $\tau \leq \sigma$ be faces of $\Sigma$. Then either the inclusion $\imath_{\tau}^{\sigma \Gamma}$ maps $\tau^{\Gamma}$ to a face of $\sigma^{\Gamma}$, or $\tau$ is independent, $\sigma$ is dependent, and $\tau^{\Gamma}$ is mapped isomorphically onto the face of $\pi\left(\sigma_{-}\right)\left(\right.$or $\left.\pi\left(\sigma_{+}\right)\right)$.

Proof. If both faces $\tau$ and $\sigma$ are independent then it follows that $\pi_{\sigma}$ and $\pi_{\tau}$ are linear isomorphisms. Then $\imath_{\tau}^{\sigma \Gamma}\left(\tau^{\Gamma}\right)$ is a face of $\sigma^{\Gamma}$. If $\tau$ and $\sigma$ are both dependent then the kernel of the projection $\pi_{\sigma}$ is contained in $\operatorname{lin}(\tau)$. Then $\pi(\tau)$ is a face of $\pi(\sigma)$. If $\tau$ is an independent face of a dependent cone $\sigma$ then $\tau$ is a face of $\sigma_{-}$ or $\sigma_{+}$. Both fans consist of independent faces of $\sigma$ and project onto the subdivisions $\pi\left(\sigma_{-}\right)$and $\pi\left(\sigma_{+}\right)$of $\pi(\sigma)$.

Definition 10.3.7. A vector $v \in \operatorname{int}\left(\sigma^{\Gamma}\right)$, where $\sigma \in \Sigma$, is $\Sigma$-stable if for any $\tau \geq \sigma$ the corresponding valuation $\operatorname{val}\left(\imath_{\sigma}^{\tau \Gamma}(v)\right)$ on $\tilde{X}_{\tau^{\Gamma}}$ is $G^{\tau}$-invariant. A vector $v \in \sigma^{\Gamma}$ is $\Sigma$-stable if there is a $\Sigma$-stable vector $v_{0} \in \operatorname{int}\left(\sigma_{0}^{\Gamma}\right)$, where $\sigma_{0} \leq \sigma$, for which $v=\imath_{\sigma_{0}}^{\sigma \Gamma}\left(v_{0}\right)$.

Proposition 10.3.8. Let $\sigma$ be a semicone in $\Sigma$. A vector $v \in \operatorname{int}(\sigma)$ is $\Sigma$-stable if $\pi_{\sigma}(v) \in \sigma^{\Gamma}$ is $\Sigma$-stable.

Proof. A stable vector $v \in \operatorname{int}(\sigma)$ determines a $G^{\tau}$-invariant valuation on any $\widetilde{X}_{\tau}$ for $\tau \geq \sigma$. Consequently, it determines a $G^{\tau}$-invariant valuation on any $\widetilde{X}_{\tau} / \Gamma_{\tau}$ and finally $\pi_{\sigma}(v)$ is $\Sigma$-stable.

Now let $v \in \operatorname{int}(\sigma)$ be an integral vector such that $\pi_{\sigma}(v) \in \operatorname{int}\left(\sigma^{\Gamma}\right)$ is stable. Then $\operatorname{val}\left(\pi_{\sigma}(v)\right)$ is $G^{\tau}$ invariant on $\widetilde{X}_{\tau} / \Gamma_{\tau}$. We have to prove that $v$ is stable, or equivalently, that for any $\tau \geq \sigma$, val $(v)$ is $G^{\tau}$-invariant on $\widetilde{X}_{\tau}$. Consider two cases:

(1) $\tau \in \operatorname{Ind}(\Sigma)$. The morphism $\tilde{j}_{\pi_{\tau}}: \tilde{X}_{\tau} \rightarrow \widetilde{X}_{\tau} / \Gamma_{\tau}=\tilde{X}_{\tau^{\Gamma}}$ is $G^{\tau}$-equivariant. The valuation $\tilde{j}_{\pi_{\tau} *}(\operatorname{val}(v))=$ $\operatorname{val}\left(\pi_{\tau}(v)\right)$ is $G^{\tau}$-invariant on $\widetilde{X}_{\tau} / \Gamma_{\tau}$. Thus for any $g \in G^{\tau}, g_{*} \tilde{j}_{\pi_{\tau} *}(\operatorname{val}(v))=\tilde{j}_{\pi_{\tau} *} g_{*}(\operatorname{val}(v))=\tilde{j}_{\pi_{\sigma} *}(\operatorname{val}(v))$. This means that $\operatorname{val}(v)$ and $g_{*}(\operatorname{val}(v))$ define the same functional on the lattice of characters $M_{\tau}^{\Gamma}$ and consequently $M_{\tau}\left(\right.$ since $\left.M_{\tau}^{\mathbf{Q}}=\left(M_{\tau}^{\Gamma}\right)^{\mathbf{Q}}\right)$. This shows by Lemma 5.1 .3 that $g_{*}(\operatorname{val}(v)) \geq \operatorname{val}(v)$ and finally by Lemma 5.1 .4 we conclude $g_{*}(\operatorname{val}(v))=\operatorname{val}(v)$.

(2) $\tau \in \operatorname{Dep}(\Sigma)$. By Lemma $10.3 .2(2), \widetilde{i_{/ K^{*}}}: \widetilde{X}_{\pi\left(\tau_{-}\right)} \rightarrow \widetilde{X}_{\pi(\tau)}$ is a $G^{\tau}$-equivariant proper birational morphism. Then the valuation $\operatorname{val}\left(v, \widetilde{X}_{\pi\left(\tau_{-}\right)}\right)=\left({\widetilde{i} / K^{*}}_{*}^{-1}\left(\operatorname{val}\left(v, \widetilde{X}_{\pi(\tau)}\right)\right)\right.$ is $G^{\tau}$-invariant. By Lemma 10.3.5 $(4)$, $\tilde{j}_{\pi_{\tau_{-}}}^{*}\left(\operatorname{val}\left(\pi(v), \widetilde{X}_{\pi\left(\tau_{-}\right)}\right)\right)=\operatorname{val}\left(\pi_{\tau_{-}}^{-1} \pi(v), \widetilde{X}_{\tau_{-}}\right)$is $G^{\tau}$-equivariant. We get $\pi_{\tau_{-}}^{-1}(\pi(v)) \in \operatorname{Inv}(\tau)$. Analogously $\pi_{\tau_{+}}^{-1}(\pi(v)) \in \operatorname{Inv}(\tau)$. By Lemma 6.2.1 this gives $v \in\left\langle\pi_{\tau_{-}}^{-1}(\pi(v)), \pi_{\tau_{+}}^{-1}(\pi(v))\right\rangle \subset \operatorname{Inv}(\tau)$.

Proposition 10.3.9. Let $\sigma \in \operatorname{Ind}(\Sigma)$ be an independent face of $\Sigma$. Then

1. All vectors in $\overline{\operatorname{par}}\left(\sigma^{\Gamma}\right) \cap \operatorname{int}\left(\sigma^{\Gamma}\right)$ are $\Sigma$-stable.

2. All vectors in $\operatorname{par}\left(\sigma^{\Gamma}\right)$ are $\Sigma$-stable.

Proof (1) Let $v \in \overline{\operatorname{par}}\left(\sigma^{\Gamma}\right) \cap \operatorname{int}\left(\sigma^{\Gamma}\right)$. We have to prove that it defines a $G^{\tau}$-invariant valuation on any $\widetilde{X}_{\pi_{\tau}(\tau)}$ for $\tau \geq \sigma$.

By Lemma 10.3.6 either $\sigma^{\Gamma}$ is a face of $\tau^{\Gamma}$ or $\tau \in \operatorname{Dep}(\Sigma)$ and $\sigma^{\Gamma}$ is a face of $\pi_{\tau}\left(\tau_{-}\right)\left(\right.$or $\left.\pi_{\tau}\left(\tau_{+}\right)\right)$. In the first case $\sigma^{\Gamma}$ is a is a $G^{\tau}$-invariant face of $\tau^{\Gamma}$. By Lemma 6.4.1 (2), val $(v)$ defines a $G^{\tau}$-invariant valuation on $\widetilde{X}_{\tau^{\Gamma}}$. In the second case $\sigma$ is a $G^{\tau}$-invariant face of $\tau_{-}$(or $\left.\tau_{+}\right)$. Consequently, the closure of the orbit $O_{\sigma^{\Gamma}}$

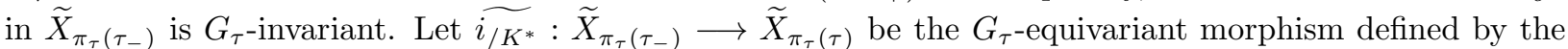
subdivision $\pi_{\tau}\left(\tau_{-}\right)$of $\pi_{\tau}(\tau)$. Then by Lemma $6.4 .1(2), \overline{\operatorname{par}}\left(\sigma^{\Gamma}\right) \cap \operatorname{int}\left(\sigma^{\Gamma}\right)$ is contained $\operatorname{Inv}\left(\pi_{\tau}(\tau)\right)$. (1).

(2) Let $v \in \operatorname{par}\left(\pi_{\sigma}(\sigma)\right)$. Then $v \in \overline{\operatorname{par}}\left(\pi_{\sigma}(\tau)\right) \cap \operatorname{int}(\tau)$ for some $\Gamma$-indecomposable face $\tau$ of $\sigma$. We apply

Lemma 10.3.10. Let $\sigma=\left\langle v_{1}, \ldots, v_{k}\right\rangle$ be a circuit in $\Sigma$ with the unique relation $\sum \alpha_{i} w_{i}=0$, where all $\alpha_{i} \neq 0$ and $w_{i}=\operatorname{prim}\left(\pi_{\sigma}\left(v_{i}\right)\right)$. Then $\mathrm{Ctr}_{-}(\sigma):=\sum_{\alpha_{i}<0} w_{i}$ and $\operatorname{Ctr}_{+}(\sigma):=\sum_{\alpha_{i}>0} w_{i}$ are $\Sigma$-stable.

Proof. We have to show that for any $\tau \geq \sigma$ the valuation $\operatorname{val}\left(\operatorname{Ctr}_{-}(\sigma), \widetilde{X}_{\tau^{\Gamma}}\right)$ is $G_{\tau^{-}}$invariant on $\widetilde{X}_{\tau^{\Gamma}}$. By Lemmas 10.2 .10 and 10.3.2(1), the face $\sigma^{-}=\left\langle v_{i}\right\rangle_{\alpha_{i}>0} \in \sigma_{-} \subset \tau_{-}$corresponds to the $G^{\tau}$-invariant 
closed subscheme of $\tilde{X}_{\tau_{-}} \subset \widetilde{X}_{\tau}$. Therefore the orbit closure scheme $\overline{O_{\pi_{\sigma}\left(\sigma^{-}\right)}} \subset \widetilde{X}_{\pi_{\tau}\left(\tau_{-}\right)}$is $G^{\tau}$-invariant. By Lemma 6.4.1 (2), applied to the subdivision $\pi_{\tau}\left(\tau_{-}\right)$of $\pi_{\tau}(\tau), v:=\mathrm{Ctr}_{-}(\sigma)$ defines a $G^{\tau}$-invariant valuation on $\widetilde{X}_{\pi_{\tau}(\tau)}$.

Lemma 10.3.11. Let $\Delta$ be a canonical subdivision of $\Sigma$.

1. If $v \in \operatorname{par}(\pi(\delta))$, where $\delta \in \Delta^{\sigma}$, is an independent cone, then $\operatorname{Mid}(v, \delta)$ is $\Sigma$-stable.

2. Let $\delta \in \Delta^{\sigma}$ be a circuit. Then $\operatorname{Mid}\left(\operatorname{Ctr}_{+}(\delta), \delta\right), \operatorname{Mid}\left(\operatorname{Ctr}_{-}(\delta), \delta\right)$ are $\Sigma$-stable.

Proof (1) There is a $\Gamma$-indecomposable cone $\delta^{\prime} \preceq \delta$ such that $v \in \operatorname{par}\left(\pi\left(\delta^{\prime}\right)\right)$. Then $\delta^{\prime} \in \Delta_{\text {stab. We apply }}^{\sigma}$ Lemma 10.3.10 and Proposition 10.3.8 to $\delta^{\prime} \in \Sigma^{\prime}=\Delta_{\text {stab }}$ to deduce that $\operatorname{Mid}(v, \delta)=\operatorname{Mid}\left(v, \delta^{\prime}\right)$ is $\Sigma^{\prime}$-stable. By Proposition 9.2.1(3), it is $\Sigma$-stable.

(2) The circuit $\delta \in \Delta^{\sigma}$ is $\Gamma$-indecomposable. Thus $\delta \in \Delta_{\text {stab }}$. We apply Lemma 10.3.10 and Proposition 10.3 .8 to $\delta \in \Sigma^{\prime}=\Delta_{\text {stab }}$ to infer that $\operatorname{Mid}\left(\operatorname{Ctr}_{+}(\delta), \delta\right), \operatorname{Mid}\left(\mathrm{Ctr}_{-}(\delta), \delta\right)$ are $\Sigma^{\prime}$-stable. Consequently, by Proposition 9.2.1(3) they are $\Sigma$-stable.

\subsection{The $\pi$-desingularization Lemma of Morelli.}

Definition 10.4.1. An independent cone $\delta \in \Delta^{\sigma}$ is $\pi$-nonsingular if $\pi_{\sigma}(\delta)$ is regular. A subdivision $\Delta$ is $\pi$-nonsingular if all independent cones in $\Delta^{\sigma}$, where $\sigma \in \Sigma$, are $\pi$-nonsingular.

Definition 10.4.2. (Morelli [49], [50], [5]) Let $\pi: N^{\mathbf{Q}+}:=N^{\mathbf{Q}} \oplus \mathbf{Q} \rightarrow N^{\mathbf{Q}}$ be the natural projection and $v=(\{0\} \times 1) \in N^{\mathbf{Q}}$. A fan $\Sigma$ in $N^{\mathbf{Q}+}$ is called a polyhedral cobordism or simply a cobordism if

1. For any cone $\sigma \in \Sigma$ the image $\pi(\sigma)$ is strictly convex (contains no line).

2. The sets of cones

$$
\Sigma_{+}:=\{\sigma \in \Sigma \mid \text { there exists } \quad \epsilon>0, \text { such that } \sigma+\epsilon \cdot v \notin|\Sigma|\}
$$

and

$$
\Sigma_{-}=\{\sigma \in \Sigma \mid \text { there exists } \epsilon>0, \text { such that } \sigma+\epsilon \cdot v \notin|\Sigma|\}
$$

are subfans of $\Sigma$ and $\pi\left(\Sigma_{-}\right):=\left\{\pi(\tau) \mid \tau \in \Sigma_{-}\right\}$and $\pi\left(\Sigma_{+}\right):=\left\{\pi(\tau) \mid \tau \in \Sigma_{+}\right\}$are fans in $N^{\mathbf{Q}}$.

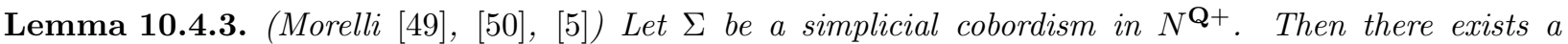
simplicial cobordism $\Delta$ obtained from $\Sigma$ by a sequence of star subdivisions such that $\Delta$ is $\pi$-nonsingular. Moreover, the sequence can be taken so that any independent and already $\pi$-nonsingular face of $\Sigma$ remains unaffected during the process. Moreover all centers of star subdivisions are of the form (1), (2) from Lemma 10.3.11.

A simple corollary of the above lemma is

Lemma 10.4.4. Let $\Sigma$ be a simplicial strictly convex $K^{*}$-semicomplex. Then there exists a canonical subdivision $\Delta$ of $\Sigma$ which is a sequence of star subdivisions such that $\Delta$ is $\pi$-nonsingular. Moreover, the sequence can be taken so that any independent and already $\pi$-nonsingular face of $\Sigma$ remains unaffected during the process. Moreover all centers are of the form (1), (2) from Lemma 10.3.11 and therefore are $\Sigma$-stable.

Lemma 10.4.5. ([72], Lemma 10, [49]) Let $w_{1}, \ldots, w_{k+1}$ be integral vectors in $\mathbf{Z}^{k} \subset \mathbf{Q}^{k}$ which are not contained in a proper vector subspace of $\mathbf{Q}^{k}$. Then

$$
\sum_{i=1}^{n}(-1)^{i} \operatorname{det}\left(w_{1}, \ldots, \check{w}_{i}, \ldots, w_{k+1}\right) \cdot w_{i}=0
$$

is the unique (up to proportionality) linear relation between $w_{1}, \ldots, w_{k+1}$.

Proof. Let $v:=\sum_{i=1}^{n}(-1)^{i} \operatorname{det}\left(w_{1}, \ldots, \check{w}_{i}, \ldots, w_{k+1}\right) \cdot w_{i}$. Then for any $i<j$,

$$
\begin{aligned}
\operatorname{det}\left(w_{1}, \ldots, \check{w}_{i}, \ldots, \check{w}_{j}, \ldots, w_{k+1}, v\right)=\operatorname{det}\left(w_{1}, \ldots, \check{w}_{i}, \ldots, w_{k+1}\right) \cdot \operatorname{det}\left(w_{1}, \ldots, \check{w}_{i}, \ldots, \check{w}_{j}, \ldots, w_{k+1}, w_{i}\right)+ \\
\quad \operatorname{det}\left(w_{1}, \ldots, \check{w}_{j}, \ldots, w_{k+1}\right) \cdot \operatorname{det}\left(w_{1}, \ldots, \check{w}_{i}, \ldots, \check{w}_{j}, \ldots, w_{k+1}, w_{j}\right)= \\
(-1)^{i}(-1)^{k-i} \operatorname{det}\left(w_{1}, \ldots, \check{w}_{j}, \ldots, w_{k+1}\right) \cdot \operatorname{det}\left(w_{1}, \ldots, \check{w}_{i}, \ldots, w_{k+1}\right)+ \\
(-1)^{j}(-1)^{k-j+1} \operatorname{det}\left(w_{1}, \ldots, \check{w}_{i}, \ldots, w_{k+1}\right) \cdot \operatorname{det}\left(w_{1}, \ldots, \check{w}_{j}, \ldots, w_{k+1}\right)=0
\end{aligned}
$$

Therefore $v \in \bigcap_{i, j} \operatorname{lin}\left\{w_{1}, \ldots, \check{w}_{i}, \ldots, \check{w}_{j}, \ldots, w_{k+1}\right\}=\{0\}$. 
Lemma 10.4.6. If $\Sigma$ is a simplicial cobordism which is $\pi$-nonsingular and $\sigma \in \Sigma$ is a circuit then $\operatorname{Mid}\left(\operatorname{Ctr}_{+}(\sigma), \sigma\right)=\operatorname{Mid}\left(\operatorname{Ctr}_{-}(\sigma), \sigma\right)$ and $\operatorname{Mid}\left(\operatorname{Ctr}_{+}(\sigma), \sigma\right) \cdot \Sigma$ is $\pi$-nonsingular.

Proof. Let $\sigma=\left\langle v_{1}, \ldots, v_{k}\right\rangle$ be a curcuit and $w_{i}:=\operatorname{prim}\left(\pi\left(\left\langle v_{i}\right\rangle\right)\right)$..

Then by Lemma 10.4.5, in the relation the unique relation $\sum \alpha_{i} w_{i}=0$ all $\alpha_{i}=\operatorname{det}\left[w_{1}, \ldots, \check{w}_{i}, \ldots, w_{k}\right]= \pm 1$ and projections of "new independent faces" are regular since they are obtained by regular star subdivisions applied to projections of "old" independent faces.

Proof of Lemma 10.4.4. First note that the local projections $\pi_{\sigma}: N_{\sigma} \rightarrow N_{\sigma}^{\Gamma}$ glue together and commute with subdivisions by Lemma 10.1 .2 and therefore the notion of $\pi$-nonsingularity is coherent. Let $\sigma_{1}, \ldots, \sigma_{k}$ denote all the faces of $\Sigma$. We shall construct by induction on $i$ a canonical $\Gamma$-subdivision $\Delta_{i}$ of $\Sigma$ such that the fan $\Delta_{i}^{\sigma_{j}}$ is $\pi$-nonsingular for all $j \leq i$. Suppose $\Delta_{i}$ is already constructed. By Lemma 10.4 .3 there exist centers $v_{1}, \ldots, v_{k}$ of the appropriate type for the cobordism $\Delta_{i}^{\sigma_{i+1}}$ in $N_{\sigma_{i+1}}$ such that $\left\langle v_{k}\right\rangle \cdot \ldots \cdot\left\langle v_{1}\right\rangle \cdot \Delta_{i}^{\sigma_{i+1}}$ is $\pi$-nonsingular. It follows from Lemma 10.3 .11 that all centers in the $\pi$-desingularization process are $\Sigma$-stable, hence by Proposition 5.4.1, $\Delta_{i+1}:=\left\langle v_{k}\right\rangle \cdot \ldots \cdot\left\langle v_{1}\right\rangle \cdot \Delta_{i}$ is canonical. By construction $\Delta_{i+1}^{\sigma_{i+1}}$ is $\pi$-nonsingular. Note that $\Delta_{i+1}^{\sigma_{j}}$ for $j<i+1$ is obtained by star subdivisions of the $\pi$-nonsingular cobordisms $\Delta_{i}^{\sigma_{j}}$ at centers of type $\operatorname{Mid}\left(\operatorname{Ctr}_{ \pm}(\delta), \delta\right)$ only and hence by Lemma 10.4.6 it remains $\pi$-nonsingular.

For completeness we give a simple proof of Lemma 10.4.3. The original proof of Morelli used different centers of star subdivisions but finally it was modified by the author ([49], [50]). The complete published proof of the lemma is given in [5] (50]). The present proof is based upon the algorithm developed in [72]. All algorithms in their final versions use the same centers of subdivisions and are closely related.

\section{Proof of Lemma 10.4.3.}

Definition 10.4.7. Let $\delta=\left\langle v_{1}, \ldots, v_{k}\right\rangle$ be a dependent cone and $w_{i}:=\operatorname{prim}\left(\pi\left(\left\langle v_{i}\right\rangle\right)\right)$. Then we shall call the relation $\sum_{i=1}^{k} r_{i} w_{i}=0$ normal if it is positively normalized and $\left|r_{i}\right|=\left|\operatorname{det}\left(w_{1}, \ldots, \check{w}_{i}, \ldots, w_{k}\right)\right|$ for $i=1, \ldots, k$. An independent cone $\sigma$ is called an $n$-cone if $|\operatorname{det}(\sigma)|=n$. A dependent cone $\sigma$ is called an $n$-cone if one of its independent faces is an $n$-cone and the others are $m$-cones, where $m \leq n$.

Lemma 10.4.8. Let $\Sigma$ be a simplicial coborism and $\delta=\left\langle v_{1}, \ldots, v_{k}\right\rangle$ be a maximal dependent cone in $\Sigma$. Set $w_{i}:=\operatorname{prim}\left(\pi\left(\left\langle v_{i}\right\rangle\right)\right)$. Let

$$
\sum_{i} r_{i} w_{i}=0
$$

where $\left|r_{i}\right|=\left|\operatorname{det}\left(w_{1}, \ldots, \check{w}_{i}, \ldots, w_{k}\right)\right|$, be its normal relation (up to sign).

(1) Let $v=\operatorname{Mid}\left(\operatorname{Ctr}_{+}(\delta), \delta\right) \in \operatorname{int}\left\langle v_{i} \mid r_{i} \neq 0\right\rangle$. Let $m_{w} \geq 1$ be the integer such that the vector $w=\frac{1}{m_{w}}\left(w_{1}+\right.$ $\left.\ldots+w_{k}\right)$ is primitive. Then the maximal dependent cones in $\langle v\rangle \cdot \delta$ are of the form $\delta_{i}=\left\langle v_{1}, \ldots, \check{v}_{i}, \ldots, v_{k}, v\right\rangle$.

1a. Let $r_{i_{0}}>0$. Then for the maximal dependent cone $\delta_{i_{0}}=\left\langle v_{1}, \ldots, \check{v_{i_{0}}}, \ldots, v_{k}, v\right\rangle$ in $\langle v\rangle \cdot \delta$, the normal relation is given (up to sign) by

$$
\sum_{r_{i}>0, i \neq i_{0}} \frac{r_{i}-r_{i_{0}}}{m_{w}} w_{i}+\sum_{r_{i}<0} \frac{r_{i}}{m_{w}} w_{i}+r_{i_{0}} w=0 .
$$

1b. Let $r_{i_{0}}<0$. Then for the maximal dependent cone $\delta_{i_{0}}=\left\langle v_{1}, \ldots, \check{v}_{i_{0}}, \ldots, v_{k}, v\right\rangle$ in $\langle v\rangle \cdot \delta$, the normal relation is given (up to sign) by

$$
\sum_{r_{i}<0}-\frac{r_{i_{0}}}{m_{w}} w_{i}+r_{i_{0}} w=0
$$

(2) Let $\sigma=\left\langle v_{i} \mid i \in I\right\rangle$ be a codefinite face of $\delta$. For simplicity assume that $r_{i} \geq 0$ for $i \in I$. Let $w=\sum_{i \in I} \alpha_{i} w_{i} \in \operatorname{par}(\pi(\sigma)) \cap \operatorname{int}(\pi(\sigma))$ and $v=\operatorname{Mid}(w, \sigma) \in \operatorname{int}(\sigma)$. Then the maximal dependent cones in $\langle v\rangle \cdot \delta$ are of the form $\delta_{i_{0}}=\left\langle v_{1}, \ldots, \check{v}_{i_{0}}, \ldots, v_{k}, v\right\rangle$, where $i_{0} \in I$.

2a. Let $i_{0} \in I$ and $r_{i_{0}}>0$. Then for the maximal dependent cone $\delta_{i_{0}}=\left\langle v_{1}, \ldots, \check{v}_{i_{0}}, \ldots, v_{k}, v\right\rangle$ in $\langle v\rangle \cdot \delta$, the normal relation is given (up to sign) by

$$
\sum_{i \in I \backslash\left\{i_{0}\right\}, r_{i}>0}\left(\alpha_{i_{0}} r_{i}-\alpha_{i} r_{i_{0}}\right) w_{i}+\sum_{i \notin I, r_{i}>0} \alpha_{i_{0}} r_{i} w_{i}+\sum_{i \in I, r_{i}=0}-\alpha_{i} r_{i_{0}} w_{i}+\sum_{r_{i}<0} \alpha_{i_{0}} r_{i} w_{i}+r_{i_{0}} w=0 .
$$

2b. Let $i_{0} \in I$ and $r_{i_{0}}=0$. For the maximal dependent cone $\delta_{i_{0}}=\left\langle v_{1}, \ldots, \check{v}_{i_{0}}, \ldots, v_{k}, v\right\rangle$, the normal relation is given (up to sign) by

$$
\sum_{i \in I \backslash\left\{i_{0}\right\}} \alpha_{i_{0}} r_{i} w_{i}+0 w_{i_{0}}=0 .
$$


Proof. It is straightforward to see that the above equalities hold. We only need to show that the relations considered are normal. For that it suffices to show that one of the coefficients is equal (up to sign) to the corresponding determinant

Comparing the coefficients of $w$ in the above relations with the normal relations from Lemma 10.4.5 we get

1a. $\operatorname{det}\left(w_{1}, \ldots, \check{w}_{i_{0}}, \ldots, w_{k}\right)=r_{i_{0}}$.

1b. The coefficient of $w$ is equal to $\left.\operatorname{det}\left(w_{1}, \ldots, \check{w}_{i_{0}}, \ldots, w_{k}\right)\right)=r_{i_{0}}$,

2 a. The coefficient of $w$ is equal to $\operatorname{det}\left(w_{1}, \ldots, \check{w}_{i_{0}}, \ldots, w_{k}\right)=r_{i_{0}}$.

$2 \mathrm{~b}$. The coefficient of $w_{i}$, where $r_{i}>0$, is equal to

$\operatorname{det}\left(w_{1}, \ldots, \check{w}_{i_{0}}, \ldots, \check{w}_{i}, \cdots, w_{k}, w\right)=\alpha_{i_{0}} \operatorname{det}\left(w_{1}, \ldots, \check{w}_{i_{0}}, \ldots, \check{w}_{i}, \cdots, w_{k}, w_{i_{0}}\right)=$ $(-1)^{k-i_{0}} \alpha_{i_{0}} \operatorname{det}\left(w_{1}, \ldots, \check{w}_{i}, \cdots, w_{k}, w\right)=(-1)^{k-i_{0}} \alpha_{i_{0}} r_{i}$.

Let $\delta=\left\langle v_{1}, \ldots, v_{k}\right\rangle$ be a dependent cone. Let $\sum r_{i} w_{i}=0$ be its normal relation.

We shall consider the following kinds of dependent cones.

Type $I$ : the cones which are pointing down: $\operatorname{Card}\left\{r_{i} \mid r_{i}<0\right\}=1$.

and the cones pointing up: $\operatorname{Card}\left\{r_{i} \mid r_{i}>0\right\}=1$.

Type $I(n, n)$ : the cones of type $I$ for which $\max \left\{\left|r_{i}\right| \mid r_{i}>0\right\}=\max \left\{\left|r_{i}\right| \mid r_{i}<0\right\}=n$.

Type $I(n, *)$ : the cones pointing down for which $\max \left\{\left|r_{i}\right| \mid r_{i}>0\right\}<n$ and $\max \left\{\left|r_{i}\right| \mid r_{i}<0\right\}=n$ and the cones pointing up for which $\max \left\{\left|r_{i}\right| \mid r_{i}<0\right\}<n$ and $\max \left\{\left|r_{i}\right| \mid r_{i}>0\right\}=n$.

Type $I(*, n)$ : the cones pointing down for which $\max \left\{\left|r_{i}\right| \mid r_{i}<0\right\}<n$ and $\max \left\{\left|r_{i}\right| \mid r_{i}>0\right\}=n$, and the cones pointing up for which $\max \left\{\left|r_{i}\right| \mid r_{i}>0\right\}<n$ and $\max \left\{\left|r_{i}\right| \mid r_{i}<0\right\}=n$.

Type $I I$ : the cones which are neither pointing down nor pointing up: $\operatorname{Card}\left\{r_{i} \mid r_{i}>0\right\}>1$ and $\operatorname{Card}\left\{r_{i} \mid r_{i}<0\right\}>1$.

Type $I I(n, n)$ : the cones of type $I I$ for which $\max \left\{\left|r_{i}\right| \mid r_{i}>0\right\}=\max \left\{\left|r_{i}\right| \mid r_{i}<0\right\}=n$.

Type $I I(n, *)$ : the cones of type $I I$ for which $\max \left\{\left|r_{i}\right| \mid r_{i} \neq 0\right\}=n$ but $\max \left\{\left|r_{i}\right| \mid r_{i}>0\right\}<n$ or $\max \left\{\left|r_{i}\right| \mid r_{i}<0\right\}<n$.

Type $I I I$ : the cones which are pointing down and pointing up at the same time:

$\operatorname{Card}\left\{r_{i} \mid r_{i}>0\right\}=\operatorname{Card}\left\{r_{i} \mid r_{i}<0\right\}=1$.

Type $I I I(n, n)$ : the cones of type $I I I$ for which $\max \left\{\left|r_{i}\right| \mid r_{i}>0\right\}=\max \left\{\left|r_{i}\right| \mid r_{i}<0\right\}=n$.

Types $I(*, *), I I(*, *), I I I(*, *)$ the cones of type $I, I I, I I I$, respectively, for which $\max \left\{\left|r_{i}\right| \mid r_{i} \neq 0\right\}<n$.

Denote by $n$ the maximum of the determinants of the projections of independent cones in the simplicial cobordism $\Sigma$. The $\pi$-desingularization algorithm consists of eliminating all dependent $n$-cones according to the above classification.

All normal relations below are considered up to sign.

Step 1. Eliminating all cones of type $I I(n, n)$

Let $\delta$ be a maximal dependent cone of type $I I(n, n)$ in $\Sigma$ with normal relation (0) (see Lemma 10.4.8). By Lemma 10.4 .8 after the star subdivision at $\operatorname{Mid}\left(\mathrm{Ctr}_{+}, \delta\right)$ all new dependent $\delta^{\prime}=\delta_{i_{0}}$ cones have normal relations of type (1a) if $r_{i_{0}}>0$ or (1b) if $r_{i_{0}}>0$. If $r_{i_{0}}>0$ in the relation (1a) the absolute values of the coefficients satisfy $\frac{1}{m_{w}}\left|r_{i}-r_{i_{0}}\right|<n$, the absolute values of the other coefficients can be $n$ only for some $r_{i}<0$ and $r_{i_{0}}$. If $r_{i_{0}}<n$ then there is no positive coefficient in (1a) which is equal to $n$. Hence $\delta^{\prime}$ can be of type $I(n, *), I(*, n), I(*, *), I I(n, *)$ or $I I(*, n)$. If $r_{i_{0}}=n$ then there is only one positive coefficient (of $w$ ) in (1a). The other coefficients are $\frac{r_{i}-r_{i_{0}}}{m_{w}}=\frac{r_{i}-n}{m_{w}} \leq 0$ and $r_{i}<0$. Thus $\delta^{\prime}$ is of the form $I(n, *)$ or $I(n, n)$.

If $\delta^{\prime}=\delta_{i_{0}}$ for $r_{i_{0}}<0$ then the normal relation is of the form (1b). If $\left|r_{i_{0}}\right|=n$ then $\delta^{\prime}$ is of the form $I(n, n)$. If $\left|r_{i_{0}}\right|<n$ then $\delta^{\prime}$ is of the form $I(*, *)$.

By taking the star subdivision we eliminate dependent cones in $\operatorname{Star}\left(\delta_{+}, \Sigma\right)$, with the normal relation proportional to (0). All the dependent $n$-cones we create are either of type $I$ or $I I(n, *)$. 
Step 2. Eliminating all cones of type $I(n, n)$. Let $\delta$ be a maximal dependent cone of type $I(n, n)$. Without loss of generality we may assume that $\delta$ is pointing down (one negative ray). Then $\delta^{+}$is of codimension 1 and $\operatorname{det}\left(\pi\left(\delta^{+}\right)\right)=n$.

Find $v_{+} \in \operatorname{par}\left(\pi\left(\delta^{+}\right)\right)$. Then $v_{+} \in \operatorname{int}\left(\pi\left(\sigma_{v}\right)\right)$, where $\sigma_{v}$ is a face of $\delta^{+}$. Thus $\sigma_{v}$ is independent.

Step 2a. Making $\sigma_{v}$ codefinite with respect to all dependent cones in $\operatorname{Star}\left(\sigma_{v}, \Sigma\right)$.

By applying certain star subdivisions we eliminate all cones for which $\sigma_{v}$ is not codefinite. Let $\delta^{\prime} \in$ $\operatorname{Star}\left(\sigma_{v}, \Sigma\right)$ be any dependent cone for which $\sigma_{v}$ is not codefinite. Note that $\delta^{\prime}$ is not of type $I I I$ since in this case the circuit consists of one positive and one negative ray and the noncodefinite face $\sigma_{v}$ containing positive and negative rays would contain a circuit, which is impossible for an independent cone. Therefore $\delta^{\prime}$ is either of type $I$ or $I I$.

Suppose first that $\delta^{\prime}=\left\langle v_{1}, \ldots, v_{k}\right\rangle$ is of type $I(n, n)$ or $I(*, n)$. Without loss of generality we may assume that there is only one coefficient $r_{i_{1}}>0$ (equal to $n$ ) in the normal relation (0) from Lemma 10.4.8(1).

Since $\sigma_{v}$ is not codefinite with respect to $\delta^{\prime}$, we have $v_{i_{1}} \in \sigma_{v}$. Note that $\operatorname{Ctr}_{+}\left(\delta^{\prime}\right)=\operatorname{prim}\left(\pi\left(\left\langle v_{i_{1}}\right\rangle\right)\right)$ and for $v:=\operatorname{Mid}\left(\operatorname{Ctr}_{+}\left(\delta^{\prime}\right), \delta^{\prime}\right)$ we have $\operatorname{prim}\left(\pi\left(\left\langle v_{i_{1}}\right\rangle\right)\right)=\operatorname{prim}\left(\pi\left(\left\langle v_{i_{1}}\right\rangle\right)\right)$. Let $w_{i}:=\operatorname{prim}\left(\pi\left(\left\langle v_{i_{1}}\right\rangle\right)\right)$ and $w:=\operatorname{prim}(\pi(\langle v\rangle))$. Then $w=w_{i_{1}}$. After the star subdivision of $\delta^{\prime}$ at $\langle v\rangle$ by Lemma 10.4.8(1a), we create a dependent cone $\delta_{i_{1}}^{\prime}$ in $\langle v\rangle \cdot \delta^{\prime}$ with the normal relation obtained from the relation (0) by replacing $w$ with $w_{i_{1}}$. Since $\sigma_{v}$ contains only negative rays of $\delta_{i_{1}}^{\prime}$ it is codefinite. We also create dependent cones $\delta_{i_{0}}^{\prime}$, where $i_{0} \neq i_{1}$, of type $I I I(I I I(n, n)$ or $I I I(*, *))$ with the normal relation $r_{i_{0}} w-r_{i_{0}} w_{i_{1}}=0$ (case $\left.1 \mathrm{~b}\right)$. The face $\sigma_{v}$ is codefinite with respect to $\delta_{i_{0}}^{\prime}$. Thus after blow-up we create one cone of the type $I(n, n)$ and cones of type $I I I(n, n)$ or $I I I(*, *)$. Since at the same time we eliminate the cone $\delta^{\prime}$, the number of maximal cones of type $I(n, n)$ remains the same. After the star subdivision $\sigma_{v}$ is codefinite with respect to all new dependent cones.

Suppose $\delta^{\prime}$ is of type $I(n, *), I(*, *) I I(n, *)$ or $I I(*, *)$. Without loss of generality we may assume that in the normal relation $(0)$ for $\delta^{\prime}$ (see Lemma 10.4.8(1)):

$\max \left\{r_{i} \mid r_{i}>0\right\} \geq \max \left\{\left|r_{i}\right| \mid r_{i}<0\right\}$.

In particular $\max \left\{\left|r_{i}\right| \mid r_{i}<0\right\}<n$.

We shall assign to such a cone $\delta^{\prime}$ for which $\sigma_{v}$ is not codefinite, the invariant $\operatorname{inv}\left(\delta^{\prime}\right):=\left(\max \left\{r_{i} \mid r_{i}>\right.\right.$ $0\}, \max \left\{\left|r_{i}\right| \mid r_{i}>0\right\}$ ), ordered lexicogragraphically.

By Lemma 10.4.8(1)), after the star subdivision at $\operatorname{Mid}\left(\operatorname{Ctr}_{+}\left(\delta^{\prime}\right), \delta^{\prime}\right)$ all new dependent cones $\delta^{\prime \prime}=\delta_{i_{0}}^{\prime}$ have normal relations of type (1a) if $r_{i_{0}}>0$ or (1b) if $r_{i_{0}}<0$.

If $r_{i_{0}}=\max \left\{r_{i} \mid r_{i}>0\right\}$ then in the relation (1a) there is only one positive coefficient of $w$ which is equal to $r_{i_{0}}$. All other coefficients: $\frac{r_{i}-r_{i_{0}}}{m_{w}}$ and $r_{i}<0$ are nonpositive with the absolute values $<n$. Thus $\delta_{i_{0}}^{\prime}$ is of the type $I(n, *)$ or $I(*, *)$. The face $\sigma_{v}$ is codefinite with respect to $\delta_{i_{0}}^{\prime}$.

If $0<r_{i_{0}}<\max \left\{r_{i} \mid r_{i}>0\right\}$ then the absolute values of the coefficients $\frac{r_{i}-r_{i_{0}}}{m_{w}}, r_{i_{0}}$ and $r_{i} \leq 0$, are $<n$. The cone $\delta_{i_{0}}^{\prime}$ is of type $I(*, *), I I(*, *), I I I(*, *)$ and $\operatorname{inv}\left(\delta_{i_{0}}^{\prime}\right)<\operatorname{inv}\left(\delta^{\prime}\right)$.

If $r_{i_{0}}<0$ then the absolute values of all coefficients in the relation (1b) are equal to $\left|r_{i_{0}}\right|<n$ or $\left|\frac{r_{i_{0}}}{m_{w}}\right|<n$ and $\delta_{i_{0}}^{\prime}$ is a dependent cone of type $I(*, *)$ for which $\sigma_{v}$ is not codefinite.

We repeat the procedure for all cones $\delta^{\prime \prime}$ for which $\sigma_{v}$ is not codefinite. The procedure terminates since for all new dependent cones for which $\sigma_{v}$ is not codefinite the invarinat $\operatorname{inv}\left(\delta^{\prime}\right)$ drops.

Step 2b. Eliminating $\delta$. Apply the star subdivision at $\langle v\rangle$ to the resulting cobordism. We eliminate all cones in $\operatorname{Star}\left(\sigma_{v}\right)$. In particular $\delta \in \operatorname{Star}\left(\sigma_{v}\right)$ will be eliminated. By Step 2(a) $\sigma_{v}$ is codefinite with respect to all dependent cones in $\operatorname{Star}\left(\sigma_{v}\right)$. All such cones $\delta^{\prime}$ can be of the type $I(n, n), I(*, n) I(n, *), I(*, *), I I(n, *)$, $I I(*, *), I I I(n, n), I I I(*, *)$.

By Lemma 10.4.8 (2) all new dependent cones after the star subdivision have normal relations of the form (2a) and (2b).

Since all the coefficients in normal relations of the form $(2 \mathrm{~b})$ are $<n$, the corresponding dependent cones are of the form $I(*, *), I I(*, *), I I I(*, *)$.

In the normal relation of the form (2a) for $\delta_{i_{0}}^{\prime}$, the absolute values of the coefficients of $w_{i}$, for $i \neq i_{1}$, are $<n$. The coefficient $r_{i_{0}}$ of $w$ is the only one which can be equal to $n$. Therefore all new dependent cones we create are of the type $I(n, *), I(*, *), I I(n, *), I I(*, *), I I I(*, *)$.

Step 3. Eliminating all cones of type $I(*, n)$ and $I I(n, *)$. 
Let $\delta=\left\langle v_{1}, \ldots, v_{k}\right\rangle$ be a cone of the type $I(*, n)$ or $I I(n, *)$.

We may assume that the positive coefficients $r_{i}$ in the normal relation are $\leq n$ and the absolute values of all negative coefficients are $<n$. Then $\delta^{+}$contains at least two positive rays.

By Lemma 10.4.8(1) after the star subdivision at $\operatorname{Mid}\left(\operatorname{Ctr}_{+}(\delta), \delta\right)$ we create new dependent $n$-cones $\delta_{i_{0}}$ only for $r_{i_{0}}=n$ in case (1a). Then by the same reasoning as in Step1, $\delta_{i_{0}}$ is of type $I(n, *)$.

Step 4. Eliminating all cones of type $\operatorname{III}(n, n)$.

Let $\delta$ be a cone of type $I I I(n, n)$. Then $\pi\left(\delta^{+}\right)=\pi\left(\delta^{-}\right)$. Find $w \in \operatorname{par}\left(\pi\left(\delta^{+}\right)\right)$and let $v:=\operatorname{Mid}\left(w, \delta^{+}\right)$. Then $v$ is in the relative interior of a face $\sigma_{v}$ of $\delta^{+}$.

Step 4a. Making $\sigma_{v}$ codefinite with respect to all dependent cones in $\operatorname{Star}\left(\sigma_{v}, \Sigma\right)$.

Let $\delta^{\prime} \in \operatorname{Star}\left(\sigma_{v}, \Sigma\right)$ be any dependent cone for which $\sigma_{v}$ is not codefinite. As in Step 2, $\delta^{\prime}$ can be only of type $I$ or $I I$. Then $\delta^{\prime}$ is of type $I(n, *), I(*, *)$ or $I I(*, *)$.

Suppose $\delta^{\prime}$ is of type $I(n, *)$. Without loss of generality we may assume that in the normal relation for $\delta^{\prime}$ there is only one positive coefficient $r_{i_{1}}$ equal to $n$ and $\left|r_{i}\right|<n$ for all negative coefficients $r_{i}$.

Since $\sigma_{v}$ is not codefinite with respect to $\delta^{\prime}$, we have $v_{i_{1}} \in \sigma_{v}$. Note that $\operatorname{Ctr}_{+}\left(\delta^{\prime}\right)=\left\langle v_{i_{1}}\right\rangle$ and for $v:=\operatorname{Mid}\left(\operatorname{Ctr}_{+}\left(\delta^{\prime}\right), \delta^{\prime}\right)$ we have $w=w_{i_{1}}$. After the star subdivision at $\left\langle v^{\prime}\right\rangle$ by Lemma 10.4.8(1) we create one dependent cone $\delta_{i_{1}}^{\prime}$ with the normal relation obtained from the normal relation for $\delta^{\prime}$ by replacing $v$ with $v_{i_{1}}$, and some dependent cones $\delta_{i_{0}}^{\prime}$, for $i_{0} \neq i_{1}$, which are of type $I I I(*, *)$ with the normal relation $r_{i_{0}} w=r_{i_{0}} w_{i_{1}}$. After the star subdivision $\sigma_{v}$ is not codefinite with respect to any new dependent cones.

Suppose $\delta^{\prime}$ is of type $I(*, *)$ or $I I(*, *)$. Without loss of generality we may assume that in the normal relation (0) for $\delta^{\prime}$ (see Lemma 10.4.8(1)):

$\max \left\{r_{i} \mid r_{i}>0\right\} \geq \max \left\{\left|r_{i}\right| \mid r_{i}<0\right\}$.

In particular $\max \left\{\left|r_{i}\right| \mid r_{i}<0\right\}<n$.

We shall assign to such a cone $\delta^{\prime}$ for which $\sigma_{v}$ is not codefinite, the invariant $\operatorname{inv}\left(\delta^{\prime}\right):=\left(\max \left\{r_{i} \mid r_{i}>\right.\right.$ $\left.0\}, \max \left\{\left|r_{i}\right| \mid r_{i}>0\right\}\right)$, ordered lexicogragraphically.

By Lemma 10.4.8(1)), after the star subdivision at $\operatorname{Mid}\left(\mathrm{Ctr}_{+}\left(\delta^{\prime}\right), \delta^{\prime}\right)$ all new dependent cones $\delta^{\prime \prime}=\delta_{i_{0}}^{\prime}$ have normal relations of type (1a) if $r_{i_{0}}>0$ or (1b) if $r_{i_{0}}<0$.

If $r_{i_{0}}=\max \left\{r_{i} \mid r_{i}>0\right\}$ then in the relation (1a) there is only one positive coefficient of $w$ which is equal to $r_{i_{0}}<n$. All other coefficients: $\frac{r_{i}-r_{i_{0}}}{m_{w}}$ and $r_{i}<0$ are nonpositive with the absolute values $<n$. Thus $\delta_{i_{0}}^{\prime}$ is of the type $I(*, *)$. The face $\sigma_{v}$ is codefinite with respect to $\delta_{i_{0}}^{\prime}$.

If $0<r_{i_{0}}<\max \left\{r_{i} \mid r_{i}>0\right\}$ then the absolute values of the coefficients $\frac{r_{i}-r_{i_{0}}}{m_{w}}, r_{i_{0}}$ and $r_{i} \leq 0$, are $<n$. The cone $\delta_{i_{0}}^{\prime}$ is of type $I(*, *), I I(*, *), I I I(*, *)$ and $\operatorname{inv}\left(\delta_{i_{0}}^{\prime}\right)<\operatorname{inv}\left(\delta^{\prime}\right)$.

If $r_{i_{0}}<0$ then the absolute values of all coefficients in the relation (1b) are equal to $\left|r_{i_{0}}\right|<n$ or $\left|\frac{r_{i_{0}}}{m_{w}}\right|<n$ and $\delta_{i_{0}}^{\prime}$ is a dependent cone of type $I(*, *)$ for which $\sigma_{v}$ is not codefinite.

We repeat the procedure for all cones $\delta^{\prime \prime}$ for which $\sigma_{v}$ is not codefinite. The procedure terminates since for all new dependent cones for which $\sigma_{v}$ is not codefinite the invarinat $\operatorname{inv}\left(\delta^{\prime}\right)$ drops.

Step 4b. Eliminating $\delta$. Apply the star subdivision at $\langle v\rangle$ to the resulting cobordism. We eliminate all cones in $\operatorname{Star}\left(\sigma_{v}\right)$. In particular $\delta \in \operatorname{Star}\left(\sigma_{v}\right)$ will be eliminated. By Step 4(a) $\sigma_{v}$ is codefinite with respect to all dependent cones in $\operatorname{Star}\left(\sigma_{v}\right)$. All such cones $\delta^{\prime}$ can be of the type $I(n, *), I(*, *), I I(*, *), \operatorname{III}(n, n)$, $\operatorname{III}(*, *)$.

By Lemma 10.4.8(2) all new dependent cones after the star subdivision have normal relations of the form (2a) and (2b).

Since all the coefficients in normal relations of the form $(2 \mathrm{~b})$ are $<n$, the corresponding dependent cones we create are of the form $I(*, *), I I(*, *), I I I(*, *)$.

If $\delta^{\prime}$ is of type $I(*, *), I I(*, *), I I I(*, *)$ then all the coefficients in normal relations of the form $(2 \mathrm{a})$ are $<n$ and the corresponding dependent cones we create are of the form $I(*, *), I I(*, *), \operatorname{III}(*, *)$.

If $\delta^{\prime}$ is of type $I(n, *)$ then we can assume that $\sigma_{v}$ is a face of $\delta^{\prime}$. If $\delta^{\prime}$ is pointing down then all positive coefficients $r_{i}$ in the normal relation for $\delta^{\prime}$ are $<n$. In the normal relation (2a) for $\delta_{i_{0}}^{\prime}$, where $r_{i_{0}}>0$, all coefficients are $<n$. If $\delta^{\prime}$ is pointing up then there is only one positive coefficient $r_{i_{1}}$. In the normal relation (2a) for $\delta_{i_{1}}^{\prime}$, the first two sums disappear and there is only one positive coefficient of $w$ equal to $r_{i_{0}} \leq n$. By 
applying the star subdivision at $\langle v\rangle$ to $\delta^{\prime}$ we create one maximal dependnent cone of type $I(n, *)$. At the same time we eliminate the cone $\delta^{\prime}$ of type $I(n, *)$.

If $\delta^{\prime}$ is of type $I I I(n, n)$ (for instance if $\delta^{\prime}=\delta$ ) then there is one positive coefficient $r_{i_{1}}=n$ and one negative coefficient $r_{i_{2}}=-n$ in the normal relation for $\delta^{\prime}$.

After the star subdivision we create only one maximal dependent cone $\delta_{i_{0}}^{\prime}$ for $i_{0}=i_{1}$ having the normal relation of the form $(2 \mathrm{a})$. In the relation (2a) the first two sums disappear and there is only one positive coefficient of $w$ equal to $r_{i_{0}} \leq n$. The absolute values of the other negative coefficients are $<n$. Thus we create one maximal dependent cone of type $I(n, *)$.

Step 5. Eliminating all dependent cones of type $I(n, *)$ and all $n$-cones which are not faces of dependent cones.

Let $\delta$ be a cone of type $I(n, *)$ or an $n$-cone which is not a face of a dependent cone.

In the first case without loss of generality we may assume that in the normal relation there is one negative coefficient equal to $-n$ and all positive coefficients are $<n$ ( $\delta$ is pointing down).

Then $\pi\left(\delta^{+}\right)=\pi(\delta)$. Find $w \in \operatorname{par}\left(\pi\left(\delta^{+}\right)\right)$and let $v=\operatorname{Mid}\left(w,\left(\delta_{+}\right)\right)$. Then $v \in \operatorname{int}\left(\sigma_{v}\right)$, where $\sigma_{v}$ is a face of $\delta_{+}$.

Step 5a. Making $\sigma_{v}$ codefinite with respect to all dependent cones in $\operatorname{Star}\left(\sigma_{v}, \Sigma\right)$. We repeat word by word the procedure in Step 4a.

Step 5b. Eliminating $\delta$. Apply the star subdivision at $\langle v\rangle$ to the resulting cobordism. We eliminate all cones in $\operatorname{Star}\left(\sigma_{v}\right)$. By Step 5(a) $\sigma_{v}$ is codefinite with respect to all dependent cones in $\operatorname{Star}\left(\sigma_{v}\right)$. All such cones $\delta^{\prime}$ can be of the type $I(n, *), I(*, *), I I(*, *), I I I(*, *)$. We repeat word by word the procedure in Step 5a excluding the last case $I I I(n, n)$. By applying the star subdivision at $\langle v\rangle$ we decrease the number of maximal cones of type $I(n, *)$ (or independent $n$-cones). All new dependent cones we create are of type $I(n, *), I(*, *), I I(*, *)$.

\section{Birational COBORDisms}

\subsection{Definition of a birational cobordism.}

Definition 11.1.1. ( 73 ): Let $X_{1}$ and $X_{2}$ be two birationally equivalent normal varieties. By a birational cobordism or simply a cobordism $B:=B\left(X_{1}, X_{2}\right)$ between them we understand a normal variety $B$ with an algebraic action of $K^{*}$ such that the sets

$$
\begin{gathered}
B_{-}:=\left\{x \in B \mid \lim _{\mathbf{t} \rightarrow 0} \mathbf{t} x \text { does not exist }\right\} \quad \text { and } \\
B_{+}:=\left\{x \in B \mid \lim _{\mathbf{t} \rightarrow \infty} \mathbf{t} x \text { does not exist }\right\}
\end{gathered}
$$

are nonempty and open and there exist geometric quotients $B_{-} / K^{*}$ and $B_{+} / K^{*}$ such that $B_{+} / K^{*} \simeq X_{1}$ and $B_{-} / K^{*} \simeq X_{2}$ and the birational map $X_{1} \rightarrow X_{2}$ is given by the above isomorphisms and the open embeddings $B_{+} \cap B_{-} / K^{*}$ into $B_{+} / K^{*}$ and $B_{-} / K^{*}$ respectively.

11.2. Collapsibility. Let $X$ be a variety with an action of $K^{*}$. Let $F \subset X$ be a set consisting of fixed points. Then we define

$$
F^{+}(X)=F^{+}=\left\{x \in X \mid \lim _{\mathbf{t} \rightarrow 0} \mathbf{t} x \in F\right\}, F^{-}(X)=F^{-}=\left\{x \in X \mid \lim _{\mathbf{t} \rightarrow \infty} \mathbf{t} x \in F\right\} .
$$

Definition 11.2.1. ( 73 ). Let $X$ be a cobordism or any variety with a $K^{*}$-action.

1. We say that a connected component $F$ of the fixed point set is an immediate predecessor of a component $F^{\prime}$ if there exists a nonfixed point $x$ such that $\lim _{\mathbf{t} \rightarrow 0} \mathbf{t} x \in F$ and $\lim _{\mathbf{t} \rightarrow \infty} \mathbf{t} x \in F^{\prime}$.

2. We say that $F$ precedes $F^{\prime}$ and write $F<F^{\prime}$ if there exists a sequence of connected fixed point set components $F_{0}=F, F_{1}, \ldots, F_{l}=F^{\prime}$ such that $F_{i-1}$ is an immediate predecessor of $F_{i}$ (see [7], Def. 1. 1$)$.

3. We call a cobordism (or a variety with $K^{*}$-action) collapsible (see also Morelli [49]) if the relation $<$ on its set of connected components of the fixed point set is an order. (Here an order is just required to be transitive.) 
Lemma 11.2.2. ([73]). A projective cobordism is collapsible.

Lemma 11.2.3. Let $B$ be a collapsible variety and $I$ be a $K^{*}$-invariant sheaf of ideals. Then the blow-up $B^{\prime}:=\mathrm{bl}_{I}(B)$ is also collapsible.

Proof. Set $\pi: B^{\prime} \rightarrow B$. For any connected fixed point component $F^{\prime}$ on $B^{\prime}$ by abuse of notation denote by $\pi\left(F^{\prime}\right)$ the fixed point component containing the image $\pi\left(F^{\prime}\right)$. Then for any $F_{0}^{\prime}$ and $F_{1}^{\prime}$ on $B^{\prime}$ the relation $F_{0}^{\prime}<F_{1}^{\prime}$ implies that either $\pi\left(F_{0}^{\prime}\right)<\pi\left(F_{1}^{\prime}\right)$ or $\pi\left(F_{0}^{\prime}\right)=\pi\left(F_{1}^{\prime}\right)$. Consider the latter case. Let $x \in F^{\prime}$ and $u_{1}, \ldots, u_{k}$ be semiinvariant generators. Then at least one of the functions $\pi^{*}\left(u_{i}\right)$, say $\pi^{*}\left(u_{i_{0}}\right)$, generates $\pi^{-1}(I) \cdot \mathcal{O}_{B^{\prime}}$ at $x$. We let $\operatorname{ord}(F)$ denote the weight of $\pi^{*}\left(u_{i_{0}}\right)$ with respect to the $K^{*}$-action. This definition does not depend on the choice of the semiinvariant generator. For any two generators $\pi^{*}\left(u_{i_{0}}\right)$ and $\pi^{*}\left(u_{i_{1}}\right)$ their quotient is a semiinvariant function which is invertible at the fixed point $x \in B^{\prime}$, which implies that it is invariant function. This definition is locally constant, which means that it does not depend on the choice of $x \in X$. Moreover the functions $\pi^{*}\left(u_{1}\right), \ldots, \pi^{*}\left(u_{k}\right)$ are sections generating $\mathcal{O}_{\pi^{-1}(U)}(-D)$, where $D$ is the exceptional divisor of $\pi$. This gives a $K^{*}$-equivariant morphism $\phi: \pi^{-1}(U) \rightarrow \mathbf{P}^{k}$ into a projective space with semiinvariant coordinates $x_{1}, \ldots x_{k}$ having the same weights as $u_{1}, \ldots, u_{k}$, say $a_{1}, \ldots, a_{k}$. We can assume that $a_{1} \leq \ldots \leq a_{k}$. Then the function $\operatorname{ord}(F)=\min \left\{a_{i} \mid x_{i}(\phi(F)) \neq 0\right\}$ coincides with the one induced on $\mathbf{P}^{k}$, and $F<F^{\prime}$ implies ord $(F)<\operatorname{ord}\left(F^{\prime}\right)$. Define $F \prec F^{\prime}$ if either $\pi(F)<\pi\left(F^{\prime}\right)$, or $\pi(F)=\pi\left(F^{\prime}\right)$ and $\operatorname{ord}(F) \leq \operatorname{ord}\left(F^{\prime}\right)$. Since $F<F^{\prime}$ implies $F \prec F^{\prime}$ it follows that $\prec$ is an order on the fixed point components on $B^{\prime}$.

\subsection{Decomposition of a birational cobordism.}

Definition 11.3.1. ( $(73])$. Let $B$ be a collapsible cobordism and $F_{0}$ be a minimal component. By an elementary collapse with respect to $F_{0}$ we mean the cobordism $B^{F_{0}}:=B \backslash F_{0}^{-}$(see section 10.3). By an elementary cobordism with respect to $F_{0}$ we mean the cobordism $B_{F_{0}}:=B \backslash \bigcup_{F \neq F_{0}} F^{+}$.

Proposition 11.3.2. (73]). Let $F_{0}$ be a minimal component of the fixed point set in a collapsible cobordism $B$. Then the elementary collapse $B^{F_{0}}$ with respect to $F_{0}$ is again a collapsible cobordism, in particular it satisfies:

1. $B_{+}^{F_{0}}=B_{+}$is an open subset of $B$,

2. $F_{0}^{-}$is a closed subset of $B$ and equivalently $B^{F_{0}}$ is an open subset of $B$.

3. $B_{-}^{F_{0}}$ is an open subset of $B^{F_{0}}$ and $B_{-}^{F_{0}}=B^{F_{0}} \backslash \bigcup_{F \neq F_{0}} F^{+}$.

4. The elementary cobordism $B_{F_{0}}$ is an open subset of $B$ such that

$$
\begin{gathered}
\left(B_{F_{0}}\right)_{-}=B_{F_{0}} \backslash F_{0}^{+}=B_{-} \\
\left(B_{F_{0}}\right)_{+}=B_{F_{0}} \backslash F_{0}^{-}=B_{-}^{F_{0}} .
\end{gathered}
$$

5. There exist good and respectively geometric quotients $B_{F_{0}} / / K^{*}$ and $B_{-}^{F_{0}} / K^{*}$ and moreover the natural embeddings $i_{-}: B_{-} \subset B_{F_{0}}$ and $i_{+}: B_{-}^{F_{0}} \subset B_{F_{0}}$ induce proper morphisms $i_{-} / K^{*}: B_{-}^{F_{0}} / K^{*} \rightarrow B_{F_{0}} / / K^{*}$ and $i_{+/ K^{*}}: B_{-} / K^{*} \rightarrow B_{F_{0}} / / K^{*}$.

We can extend the order $<$ from Definition 11.2.1 to a total order.

As a corollary from the above we obtain:

Proposition 11.3.3. Let $F_{1}<\ldots<F_{k}$ denote the connected fixed point components of a cobordism B.

Set $B_{i}:=B \backslash\left(\bigcup_{F_{j}<F_{i}} F_{j}^{-} \cup \bigcup_{F_{j}>F_{i}} F_{j}^{+}\right)$.

Then

1. $\left(B_{1}\right)_{-}=B_{-},\left(B_{k}\right)_{+}=B_{+}$.

2. $\left(B_{i+1}\right)_{-}=\left(B_{i}\right)_{+}$.

3. There is a factorization

$B_{-} / K^{*}=\left(B_{1}\right)_{-} / K^{*}-\rightarrow\left(B_{1}\right)_{+} / K^{*}=\left(B_{2}\right)_{-} / K^{*}-\rightarrow \ldots\left(B_{k-1}\right)_{+} / K^{*}=\left(B_{k}\right)_{-} / K^{*}-\rightarrow\left(B_{k}\right)_{+} / K^{*}=B_{+} / K^{*}$. 


\subsection{Existence of a smooth birational cobordism.}

Proposition 11.4.1. (see also [73) Let $\phi: Y \rightarrow X$ be a birational projective morphism between smooth complete varieties over an algebraically closed field of characteristic 0 . Let $U \subset X, Y$ be an open subset , where $\phi$ is an isomorphism.

1. There exists a smooth collapsible cobordism $B=B(X, Y)$ between $X$ and $Y$ such that $B \supset U \times K^{*}$

2. If $D_{X}:=X \backslash U$ and $D_{Y}:=Y \backslash U$ are divisors with normal crossings then there exists a cobordism $\tilde{B}$ between $\tilde{X}$ and $\tilde{Y}$ such that

- $\tilde{X}$ and $\tilde{Y}$ are obtained from $X$ and $Y$ by a sequence of blow-ups at centers which have normal crossings with components of the total transforms of $D_{X}$ and $D_{Y}$ respectively.

- $U \times K^{*} \subset \tilde{B}$ and $\tilde{B} \backslash\left(U \times K^{*}\right)$ is a divisor with normal crossings.

Proof. (1) If $X$ and $Y$ are projective the construction of $B$ is given in Proposition 2 of 773 ; in general we use the construction of D.Abramovich (Remark after Proposition 2 in [73]). $B$ is an open subset of $\bar{B}$ where $\bar{B}$ is obtained from $X \times \mathbf{P}^{1}$ by a sequence of blow-ups at ideals, hence by a single blow-up of an ideal. By Lemma 11.2.3, $\bar{B}$ is collapsible. Consequently $B$ is a collapsible cobordism.

(2) The sets $B_{-}$and $B_{+}$are locally trivial $K^{*}$-bundles with projections $\pi_{-}: B_{-} \rightarrow B_{-} / K^{*} \simeq X$ and $\pi_{+}: B_{+} \rightarrow B_{+} / K^{*} \simeq Y$. Let $Z:=B \backslash\left(U \times K^{*}\right)$. Then $Z \cap B_{-}$and $Z \cap B_{+}$are normal crossing divisors and $\pi_{-}\left(Z \cap B_{-}\right)=D_{X}$ and $\pi_{+}\left(Z \cap B_{+}\right)=D_{Y}$. Let $f: \tilde{B} \rightarrow B$ be a canonical principalization of $I_{Z}$ (see Hironaka [29], Villamayor [71] and Bierstone-Milman [8]). Let $f_{+}: f^{-1}\left(B_{+}\right) \rightarrow B_{+}\left(\right.$resp. $\left.f_{-}: f^{-1}\left(B_{-}\right) \rightarrow B_{-}\right)$be its restriction. By functoriality $f_{+}$(resp. $\left.f_{-}\right)$is a canonical principalization of $B_{+}$(resp. $B_{-}$) which commutes with a canonical principalization $\tilde{Y}$ of $I_{D_{Y}}$ on $Y$ (resp. $\tilde{X}$ of $I_{D_{X}}$ on $D_{X}$ ) In particular all centers are $K^{*}$-invariant of the form $\pi_{+}^{-1}(C)$ (resp. $\pi_{-}^{-1}(C)$ ), where $C$ have normal crossings with components of the total transform of $D_{Y}\left(\operatorname{resp} . D_{X}\right)$.

\section{WEAK FACTORIZATION THEOREM}

\subsection{Construction of a $\pi$-regular cobordism.}

Definition 12.1.1. $\quad 1$. We say that a cobordism $B$ is $\pi$-regular if for any $x$ in $B$ which is not a fixed point of the $K^{*}$-action in $B$ there exists an affine invariant neighborhood $U$ without fixed points such that $U / K^{*}$ is smooth.

2. We call a cobordism $B K^{*}$-stratified if it is a $K^{*}$-stratified toroidal variety.

Proposition 12.1.2. Let $(B, S)$ be a smooth stratified cobordism between smooth varieties $X$ and $X^{\prime}$. There exists a $K^{*}$-toroidal modification $\tilde{B}$ of $B$ obtained as a sequence of blow-ups of stable valuations such that $\tilde{B}$ is a $\pi$-regular $K^{*}$-stratified cobordism between $X$ and $X^{\prime}$. Moreover if $U \subset X$ is an open affine invariant fixed point free subset such that $U / K^{*}$ is smooth then all centers of blow-ups are disjoint from $U$.

Proof. By Lemma 4.8.3 we can associate with $(B, S)$ a $K^{*}$-semicomplex $\Sigma$. Since for any fixed point component $F$ in $B$ the sets $F^{+}$and $F^{-}$are not dense it follows that $\Sigma_{S}$ is strictly convex. By Lemma 10.4 .4 there is a $K^{*}$-canonical subdivision $\Delta$ of $\Sigma$ which is $\pi$-nonsingular and does not affect any $\pi$-nonsingular cones in $\Sigma$. By Theorem 6.6.1, $\Delta$ corresponds to a $K^{*}$-stratified cobordism between $X$ and $Y$.

\subsection{Factorization determined by a $\pi$-regular cobordism.}

Proposition 12.2.1. Let $(B, R)$ be a $\pi$-regular collapsible $K^{*}$-stratified cobordism between smooth stratified toroidal varieties $\left(X, S_{X}\right)=\left(B_{-} / K^{*},\left(R \cap B_{-}\right) / K^{*}\right),\left(Y, S_{Y}\right)=\left(B_{+} / K^{*},\left(R \cap B_{+}\right) / K^{*}\right)$. Let $B_{i}$ be elementary cobordisms as in Proposition 11.3.9. Then there exists a sequence of smooth stratified toroidal varieties $\left(X_{i}, S_{i}\right):=\left(\left(B_{i}\right)_{-} / K^{*},\left(R \cap\left(B_{i}\right)_{-}\right) / K^{*}\right)$ and a factorization

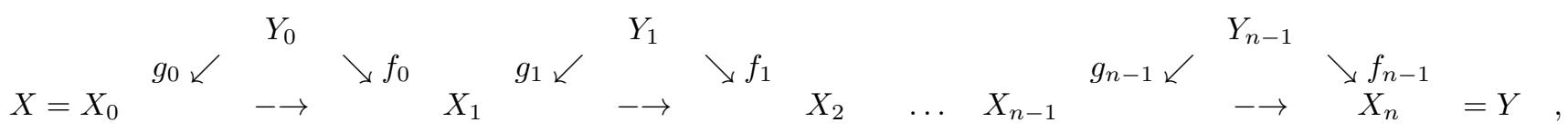

where each $X_{i}$ is a smooth variety and $f_{i}: Y_{i} \rightarrow X_{i+1}$ and $g_{i}: Y_{i} \rightarrow X_{i}$ for $i=0, \ldots, n-1$ are blow-ups at smooth centers which have normal crossings with the closures of strata in $S_{i}$ (resp. $S_{i+1}$ ).

Proof. Follows from the following Lemma 12.2.2 and Proposition 11.3.3. 
Lemma 12.2.2. Let $(B, R)$ be a $\pi$-regular elementary $K^{*}$-stratified cobordism. Then $X=B_{-} / K^{*}$ and $Y=B_{+} / K^{*}$ are smooth stratified toroidal varieties with stratifications $S_{X}$ and $S_{Y}$ induced by $R \cap B_{+}$and $R \cap B_{-}$and there exists a smooth variety $Z$, obtained by a blow-up at a smooth center $C_{X}$ (resp. $\left.C_{Y}\right)$, having only normal crossings with closures of strata in $S_{X}$ (resp. $\left.S_{X}\right)$.

Proof. For any $X$ let $\tilde{X}$ denote its normalization.

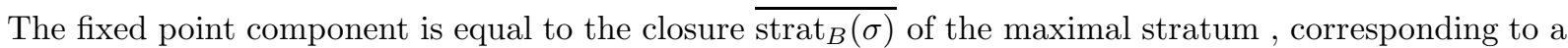
circuit $\sigma$.

Consider the commutative diagram

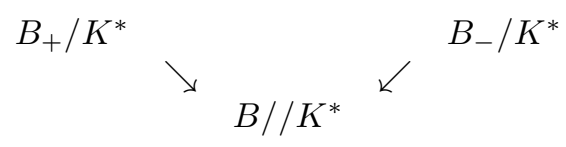

This diagram can be completed to

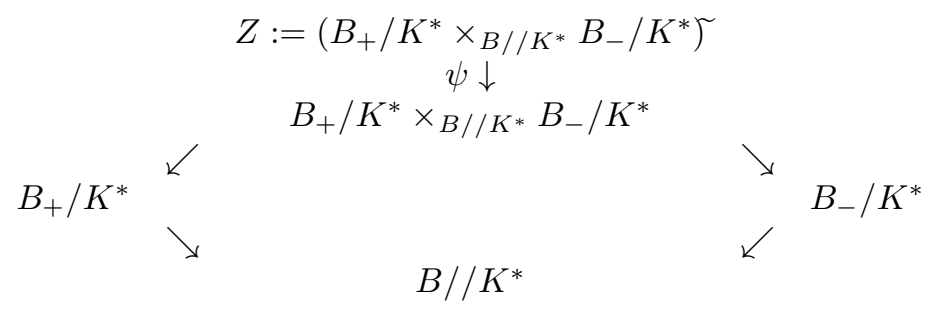

where the morphism $\psi$ is given by the normalization.

For any $x \in \overline{\operatorname{strat}(\sigma)}$ find $\tau \geq \sigma$ such that $x \in \operatorname{strat}(\tau)$. Let $\phi_{\tau}: U_{\tau} \rightarrow X_{\tau}$ be the relevant chart.

The above diagram defines locally a diagram

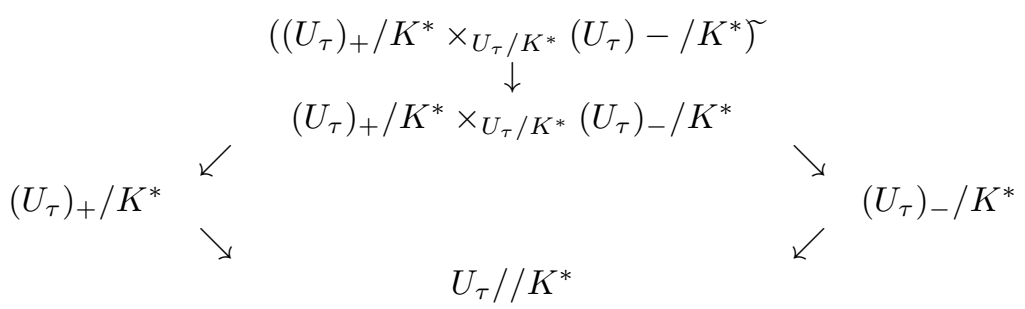

This diagram is a pull-back via a smooth morphism $\phi_{\tau / K^{*}}: U_{\tau} / / K^{*} \rightarrow X_{\tau} / K^{*}$ of the diagram of toric varieties

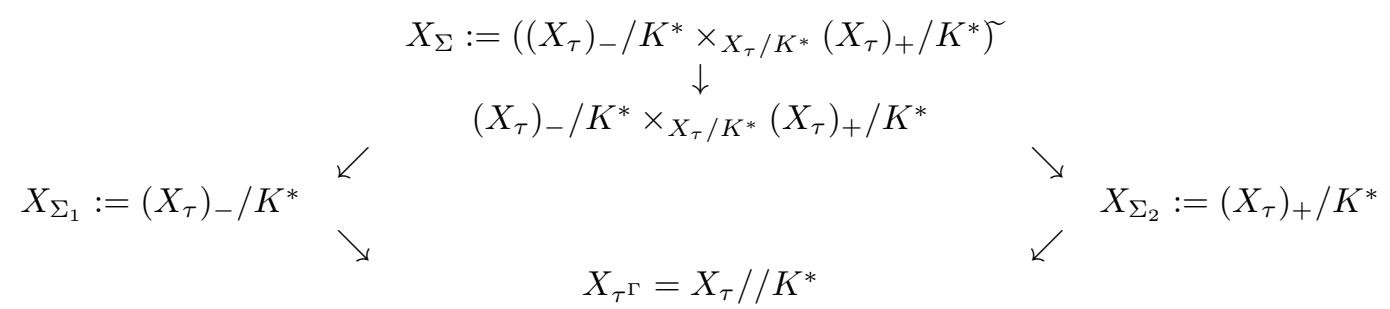

It follows from the universal property of the fiber product that $X_{\Sigma}$ is a normal toric variety whose fan consists of the cones $\left\{\tau_{1} \cap \tau_{2} \mid \tau_{1} \in \Sigma_{1}, \tau_{2} \in \Sigma_{2}\right\}$.

The cone $\tau$ is $\pi$-nonsingular and by Lemma 10.4.5 we can write the only dependence relation of $\tau^{\Gamma}=$ $\left\langle v_{1}, \ldots, v_{k}, w_{1}, \ldots, w_{m}, q_{1}, \ldots, q_{l}\right\rangle$, where $\left\langle v_{1}, \ldots, v_{k}, w_{1}, \ldots, w_{m}\right\rangle$ is a curcuit, as follows:

$$
e:=v_{1}+\ldots+v_{k}=w_{1}+\ldots+w_{m} .
$$

Finally, $\Sigma=\langle e\rangle \cdot \Sigma_{1}$ and $\Sigma=\langle e\rangle \cdot \Sigma_{2}$ are regular star subdivisions.

We have shown that the morphisms $Z \rightarrow X$ and $Z \rightarrow Y$ defined by the diagram (*) are blow-ups at smooth centers. These centers have normal crossings with closures of strata since locally the centers and the closures of strata are determined by the closures of orbits on a smooth toric variety. 


\subsection{Nagata's factorization.}

Lemma 12.3.1. (Nagata) Let $X \supset U \subset Y$ be two complete varieties over an algebraically closed field, containing an open subset $U$. Then there exists a variety $Z$ which is simultaneously a blow-up of a coherent sheaf of ideals $\mathcal{I}_{X}$ on $X$ and a blow-up of a coherent sheaf of ideals $\mathcal{I}_{Y}$ on $Y$. Moreover the supports of $\mathcal{I}_{X}$ and $\mathcal{I}_{Y}$ are disjoint from $U$

The above lemma is a refinement of the original lemma of Nagata

Definition 12.3.2. (Nagata [54) Let $X_{i} \supset U$, for $i=1, \ldots, n$ be complete varieties over an algebraically closed field, containing an open subset $U$. By the join $X_{1} * \ldots * X_{k}$ we mean the closure of the diagonal $\Delta(U) \subset X_{1} \times \ldots \times X_{k}$.

Lemma 12.3.3. (Nagata [54]) Let $X \supset U \subset Y$ be two complete varieties over an algebraically closed field, containing an open subset $U$. Then there exist coherent sheaves of ideals $\mathcal{I}_{1}, \ldots, \mathcal{I}_{k}$ on $X$ with supports disjoint from $U$ and blow-ups $X_{1}=\mathrm{bl}_{\mathcal{I}_{1}} X, \ldots, X_{k}=\mathrm{bl}_{\mathcal{I}_{k}} X$ such that the join $X_{1} * \ldots * X_{k}$ dominates $Y$.

Proof of Lemma 12.3.1 Let $X_{i}$ be as in the assertion of Lemma 12.3.3. Note that $X_{1} \times \ldots \times X_{k}$ is projective over $X \times \ldots \times X$. This implies that $X_{1} * \ldots * X_{k}$ is projective over $\Delta(X) \simeq X$. Thus there exists a sheaf of ideals $\mathcal{J}$ on $X$ such that $X_{1} * \ldots * X_{k} \rightarrow X$ is the blow-up at $\mathcal{J}$. By Nagata and the above find a blow-up $X^{\prime}$ of $X$ at $\mathcal{J}$ such that $X^{\prime}$ dominates $Y$. Denote the blow-up by $\phi: X^{\prime} \rightarrow Y$. Analogously find a blow-up $Z$ of $Y$ at $\mathcal{I}_{Y}$ such that $Z$ dominates $X^{\prime}$. Thus $Z \rightarrow X^{\prime}$ is a blow-up at $\phi^{-1}\left(\mathcal{I}_{Y}\right)$ and $Z \rightarrow X$ is a composite of blow-ups and consequently a single blow-up at some sheaf of ideals $\mathcal{I}_{X}$.

12.4. Proof of the Weak Factorization Theorem. Step 1 By Nagata's Lemma 12.3.1 we find a $Z^{\prime}$ obtained from $X$ and $Y$ respectively by blow-ups at centers disjoint from $U$. If $X$ and $Y$ are projective we can take $Z^{\prime}$ to be the graph of $\phi: X-\rightarrow Y$. Let $Z^{\prime \prime}$ be a resolution of singularities of $Z^{\prime}$ and $Z$ be a canonical principalization of the ideal of the set $Z^{\prime \prime} \backslash U$ (this is needed for part (2) of the theorem only). Then $Z \backslash U$ is a divisor with normal crossings. It suffices to prove the theorem for the projective morphisms $Z \rightarrow X$ (or $Z \rightarrow Y)$.

Step 2 By Proposition 11.4.1 there exists a smooth collapsible cobordism $B=B(Z, X)$. If $D_{X}:=X \backslash U$ and $D_{Z}:=Z \backslash U$ are divisors with normal crossings then there exists a cobordism $\tilde{B}$ between $\tilde{X}$ and $\tilde{Y}$ as in the proposition such that $\tilde{B} \backslash\left(U \times K^{*}\right)$ is a normal crossings divisor. For simplicity denote $\tilde{X}, \tilde{Z}, \tilde{B}$ by $X, Z, B$ respectively.

Step 3 By Lemma 4.2.1 there exists a $K^{*}$-invariant stratification $S$ on $B$ such that $(B, S)$ is a $K^{*}$-stratified toroidal variety. If $\overline{B \backslash}\left(U \times K^{*}\right)$ is a normal crossings divisor then the components of this divisor are the closures of some strata in $S$.

Step 4 By Proposition 12.1.2 we can find a $\pi$-regular collapsible $K^{*}$-stratified cobordism $(\bar{B}, \bar{R})$ between $X$ and $Z$ by applying a sequence of blow-ups at stable valuations to $(B, S)$ and using the combinatorial algorithm. The subsets $B_{+}=\bar{B}_{+}$and $B_{-}=\bar{B}_{-}$are unaffected.

Step 5 By Proposition 12.2.1 the $\pi$-regular cobordism $(\bar{B}, \bar{R})$ determines a factorization of the map $Z \rightarrow X$ into a sequence of blow-ups and blow-downs. All centers have only normal crossings with closures of the induced strata on intermediate varieties.

\section{Comparison With ANOTher Proof. TORIFicAtion And Stable Support}

13.1. Comparison with another proof. In [4] another proof of the Weak Factorization Theorem is given , where the theorem is stated an proved in a more general version. In particular the assumption of the base field to be algebraically closed was removed. The theorem was also formulated and proved for bimeromorphic

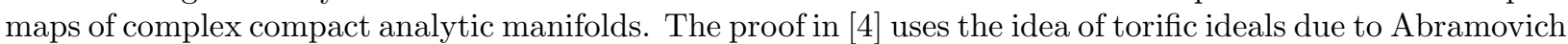
and De Jong. The idea of the proof is to consider an ideal on a smooth cobordism whose blow-up determines a structure of toroidal embedding compatible with the $K^{*}$-action. Such an ideal, called "torific", and the the blow-up procedure, called torification were introduced by D.Abramovich and J.de Jong. The torific ideal is defined in an invariant neighborhood $U$ of the fixed point component of the smooth variety with $K^{*}$-action. Let $u_{1}, \ldots, u_{k}$ denote the semiinvariant parameters with weights $a_{1}, \ldots, a_{k}$. We define the torific ideal to be $I=I_{a_{1}} \cdot \ldots \cdot I_{a_{k}}$, where $I_{a}$ denotes the ideal generated by all semiinvariant functions of weight $a$. The 
blowing-up of the torific ideal induces a structure of toroidal embedding, locally on each elementary piece of cobordism. Torification allows one to pass to the category of toroidal embeddings, where the factorization problem has already been solved using combinatorial algorithms. However the torific ideal can be constructed only locally on elementary cobordisms. Hence after the torification is done on each elementary cobordism some glueing procedure should be applied. Using canonical principalizations of torific ideals and canonical resolution of singularities of intermediate varieties gives a factorization on an elementary cobordism between canonical resolution of singularities. Patching these factorizations together one gets a decomposition of the given birational map.

13.2. Torification and stable support. The notion of torification can be understood and generalized on the ground of the theory of stratified toroidal varieties.

Definition 13.2.1. Let $(X, S)$ be an oriented stratified toroidal variety (with or without group action). By a stable ideal $\mathcal{I}$ we mean a coherent sheaf of ideals $\mathcal{I}$ satisfying the following conditions :

1. The support of $\mathcal{I}$ is a union of strata in $S$.

2. For any points $x_{1}$ and $x_{2}$ in a stratum $s$ and any isomorphism $\phi: \widehat{X}_{x_{1}} \rightarrow \widehat{X}_{x_{2}}$ preserving strata and

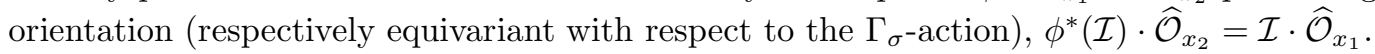

Proposition 13.2.2. Let $\mathcal{I}$ be a stable ideal on an oriented stratified toroidal variety $(X, S)$. Then for any $\sigma \in \Sigma$ there is a $G^{\sigma}$-invariant toric ideal $I_{\sigma}$ on $X_{\sigma}$ such that

1. The induced ideal on $\tilde{X}_{\sigma}$ is $G^{\sigma}$-invariant.

2. For any chart $\phi_{\sigma}: U \rightarrow X_{\sigma}, \mathcal{I}_{U}=\phi_{\sigma}^{*}\left(I_{\sigma}\right)$.

3. For any $\tau \leq \sigma, I_{\tau} \cdot \mathcal{O}_{x, X_{\sigma}}$ is the restriction of $I_{\sigma}$ to the open subset $X_{\left(\tau, N_{\sigma}\right)} \subset X_{\sigma}$.

One can rephrase the above proposition in combinatorial language, using the correspondence between toric ideals $\mathcal{I}$ and $\operatorname{ord}(\mathcal{I})$-functions in [39] and Lemma 7.6.4.

Proposition 13.2.3. Let $(X, S)$ be an oriented stratified toroidal variety with an associated oriented semicomplex $\Sigma$. The stable complete coherent sheaves of ideals $\mathcal{I}$ on $(X, S)$ are in $1-1$ correspondence with collections of functions $\operatorname{ord}\left(I_{\sigma}\right), \sigma \in \Sigma$, satisfying:

1. $\operatorname{ord}\left(I_{\sigma}\right): \sigma \rightarrow \mathbb{Q}$ is a convex piecewise linear function $\operatorname{ord}\left(I_{\sigma}\right)\left(N_{\sigma}\right) \rightarrow \mathbf{Z}$.

2. Denote by $\Delta\left(I_{\sigma}\right)$ the subdivision of $\sigma$ into the maximal cones where $\operatorname{ord}\left(I_{\sigma}\right)$ is linear. Then $\operatorname{Vert}\left(\Delta\left(I_{\sigma}\right)\right) \subset$ $\operatorname{stab}(\sigma) \cup \operatorname{Vert}(\bar{\sigma})$, and $\operatorname{ord}\left(I_{\sigma}\right)(v)=0$ for any $v \in \operatorname{Vert}\left(\Delta\left(I_{\sigma}\right)\right) \backslash \operatorname{stab}(\sigma)$.

3. For any $\tau \leq \sigma$, the restriction of $\operatorname{ord}\left(I_{\sigma}\right)$ to $\tau$ is equal to $\operatorname{ord}\left(I_{\tau}\right)$.

It follows that the functions $\operatorname{ord}\left(I_{\sigma}\right)$ patch together and give a function $\operatorname{ord}(\mathcal{I})$ on the totality of vectors in faces of $\Sigma$.

Lemma 13.2.4. Denote by $\Delta(\mathcal{I})$ the subdivision of $\Sigma$ obtained by glueing the subdivisions $\Delta\left(I_{\sigma}\right)$. Then $\Delta(\mathcal{I})$ is a canonical subdivision of $\Sigma$ correspondinding to the blow-up of $(X, S)$ at $\mathcal{I}$.

Definition 13.2.5. Let $(X, S)$ be an oriented stratified toroidal variety (with or without group action). Then an ideal $\mathcal{I}$ is called torific if it is stable and the normalization of its blow-up is a canonical toroidal morphism $(Y, R) \rightarrow(X, S)$ such that $(Y, R)$ is a toroidal embedding. Such a blow-up is called a torification.

Lemma 13.2.6. Let $(X, S)$ be an oriented stratified toroidal variety with an associated semicomplex $\Sigma$. Then a stable ideal $\mathcal{I}$ is torific iff any $\sigma \in \Delta(\mathcal{I})$ whose relative interior intersects the stable support $\operatorname{Stab}(\Sigma)$, is contained in $\operatorname{Stab}(\Sigma)$.

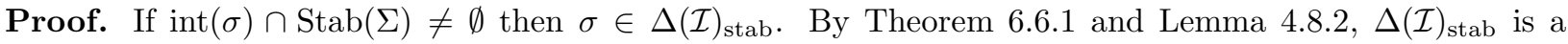
complex corresponding to the toroidal embedding. Hence all vectors from $\sigma$ are stable.

Torification is not always possible. However we can perform torification locally in the following sense.

Proposition 13.2.7. Let $(X, S)$ be an oriented stratified toroidal variety with an associated oriented semicomplex $\Sigma$. Then for any $\sigma \in \Sigma$ there is a $G^{\sigma}$-invariant ideal

$$
I_{\sigma}:=\left(G^{\sigma} \cdot u_{1}\right) \cdot \ldots \cdot\left(G^{\sigma} \cdot u_{k}\right),
$$

where $u_{1}, \ldots, u_{k}$ are toric paramaters on $\tilde{X}_{\sigma}$ such that $\Delta\left(I_{\sigma}\right)$ contains the face $\operatorname{Inv}(\sigma)$. In particular

$$
\operatorname{Inv}(\sigma)=\operatorname{conv}\left(\left(\operatorname{Vert}\left(\Delta\left(I_{\sigma}\right)\right) \backslash \operatorname{Vert}(\sigma)\right) \cup \operatorname{Vert}^{\text {semic }}(\sigma)\right),
$$


where $\operatorname{Vert}^{\text {semic }}(\sigma)$ denotes the set of 1-dimensional faces in the semicone $\sigma$.

Proof. Denote by $v_{\nu}$ the vector corresponding to a toric valuation $\nu$. Note that a toric valuation $\nu$ is $G^{\sigma}-$ invariant iff $\left.\nu\left(g_{*}\left(u_{i}\right)\right)=\nu\left(u_{i}\right)\right)$ for any $i=1, \ldots, k$ and $g \in G^{\sigma}$. It follows from Lemma 5.1.4 that the latter statement is equivalent to the following one: $\nu\left(g_{*}\left(u_{i}\right)\right) \geq \nu\left(u_{i}\right)$ for any $g \in G^{\sigma}$. This can be reformulated as follows: $\nu$ is $G^{\sigma}$-invariant iff $\operatorname{ord}\left(I_{\sigma}\right)\left(v_{\nu}\right)=\nu\left(u_{1} \cdot \ldots \cdot u_{k}\right)$. Finally, $\operatorname{Inv}(\sigma)$ is a set where ord $\left(I_{\sigma}\right)$ is $\operatorname{linear}$ and equal to the functional of $u_{1} \cdot \ldots \cdot u_{k}$. Hence $\operatorname{Inv}(\sigma)$ occurs as a cone in $\Delta\left(I_{\sigma}\right)$. All vertices of this cone belong to $\operatorname{Inv}(\sigma)$ and hence they have to be in the form as in the proposition.

Remark. The above proposition generalizes the construction of torific ideal due to Abramovich and de Jong ( 2 ).

13.3. Computing the stable support. Proposition 13.2 .7 provides a method of computing the stable support of semicomplexes.

Example 13.3.1. Let $(X, S)$ be a toroidal variety with an isolated singularity $x_{1} x_{2}=x_{3} x_{4}$ as in Example 4.10.7. The associated semicomplex $\Sigma$ consists of the cone over a square and the apex of this cone. Then $G^{\sigma} \cdot x_{i}=\left(x_{1}, x_{2}, x_{3}, x_{4}\right)$ is the ideal $m_{p}$ of the singular point $p$. Hence the torific ideal $I_{\sigma}$, where $s=\{p\}$, is equal to $m_{p}^{4} . \Delta\left(I_{\sigma}\right)$ is the star subdivision at the ray $\varrho$ over the centre of the square. By Proposition 13.2 .7 ,

$$
\operatorname{Stab}(\Sigma)=\operatorname{Inv}(\sigma)=\varrho .
$$

Example 13.3.2. Let $(X, S)$ be a smooth stratified toroidal variety of dimension 3 with the stratification determined by two curves $l_{1}$ and $l_{2}$ meeting transversally at some point $p$. The associated semicomplex $\Sigma$ consists of the regular 3-dimensional cone $\sigma=\left\langle w_{1}, w_{2}, w_{3}\right\rangle$ and its two 2-dimensional faces $\tau_{1}=\left\langle w_{1}, w_{3}\right\rangle$ and $\tau_{2}=\left\langle w_{2}, w_{3}\right\rangle$. Set $v_{1}:=w_{1}+w_{3}, v_{2}:=w_{2}+w_{3}, v:=w_{1}+w_{2}+w_{3}$. Write $l_{1}: x_{1}=x_{3}=0$, $l_{2}: x_{2}=x_{3}=0$. Then $\left(G^{\sigma} \cdot x_{1}\right)=I_{l_{1}}=\left(x_{1}, x_{3}\right),\left(G^{\sigma} \cdot x_{2}\right)=\left(x_{2}, x_{3}\right),\left(G^{\sigma} \cdot x_{3}\right)=I_{l_{1} \cup l_{2}}=\left(x_{3}, x_{1} x_{2}\right)$. Then $\Delta\left(I_{\sigma}\right)$ is determined by two star subdivisions: $\left\langle v_{1}\right\rangle \cdot \sigma,\left\langle v_{2}\right\rangle \cdot \sigma$, and the subdivision $\Sigma_{0}$ of $\sigma$ determined by $\operatorname{ord}\left(\left(x_{3}, x_{1} x_{2}\right)\right):=\min \left(w_{3}^{*}, w_{1}^{*}+w_{2}^{*}\right)$. The latter consists of two cones separated by the 2-dimensional face $\left\langle v_{1}, v_{2}\right\rangle$. Then $\operatorname{Inv}(\sigma)=\left\langle v_{1}, v_{2}, v\right\rangle$. Also $\operatorname{Inv}\left(\tau_{i}\right)=\left\langle v_{i}\right\rangle$. Finally,

$$
\operatorname{Stab}(\Sigma)=\operatorname{Inv}(\sigma)=\left\langle v_{1}, v_{2}, v\right\rangle .
$$

The above considerations can be generalized to any regular semicomplex. We shall skip the details of the proof.

Proposition 13.3.3. Let $\Sigma$ be a regular semicomplex. For any cone $\sigma=\left\langle v_{1}, \ldots, v_{k_{\sigma}}\right\rangle$ set $v_{\sigma}:=v_{1}+\ldots+v_{k_{\sigma}}$. Then

$$
\operatorname{stab}(\sigma)=\bigoplus_{\tau \leq \sigma} \mathbf{Q} \cdot v_{\tau}
$$

This formula can be extended to any oriented simplicial semicomplex.

Conjecture 13.3.4. Let $\Sigma$ be an oriented simplicial semicomplex. For any $\sigma \in \Sigma$ let $V_{\sigma}$ denote the set of all the minimal internal vectors of $\sigma$ and $W_{\sigma}:=\bigcup_{\tau \leq \sigma} V_{\tau}$. Then

$$
\operatorname{stab}(\sigma)=\bigoplus_{v \in W_{\sigma}} \mathbf{Q} \cdot v
$$

\section{REFERENCES}

[1] S. Abhyankar, On the valuations centered in a local domain, Amer. J. Math. 78 (1956), 321-348.

[2] D. Abramovich and A. J. de Jong, Smoothness, semistability, and toroidal geometry, J. Alg. Geom. 6, 1997, $789-801$.

[3] D. Abramovich and K. Karu, Weak semistable reduction in characteristic 0, Invent. math. 139 (2000), 241-273.

[4] D. Abramovich , K. Karu , K. Matsuki, J. Włodarczyk, Torification and factorization of birational maps, math. AG/9904135

[5] D. Abramovich, K. Matsuki and S. Rashid, A note on the factorization theorem of toric birational maps after Morelli and its toroidal extension, Tohoku Math. J. (2) 51 (1999), 489-537.

[6] D. Abramovich and J. Wang, Equivariant resolution of singularities in characteristic 0, Math. Res. Letters 4, 427-433 (1997).

[7] A. Białynicki-Birula,J. Święcicka, Complete quotients by algebraic torus actions, Lecture Notes in Mathematics 956, Springer (1982) ,10-21. 
[8] E. Bierstone and D. Milman, Canonical desingularization in characteristic zero by blowing up the maximum strata of a local invariant, Invent. Math. 128, 1997, 207-302.

[9] F.Bittner, The universal Euler characteristic for varieties of characteristic zero, preprint math. AG/0111062.

[10] A.Borel Linear Algebraic Groups, W.A.Benjamin, New-York, Amsterdam, 1969.

[11] Bourbaki Commutative algebra, Hermann Ch. 3, 1961.

[12] M. Brion and C. Procesi, Action d'un tore dans une variété projective, in Operator algebras, unitary representations, enveloping algebras, and invariant theory (Paris, 1989), 509-539, Progr. Math., 92, Birkhäuser, Boston, 1990.

[13] C. Christensen, Strong domination/weak factorization of three dimensional regular local rings, J. Indian Math. Soc. 45, 1981, 21-47.

[14] A. Corti, Factorizing birational maps of 3-folds after Sarkisov, J. Alg. Geom. 4, 1995, 23-254.

[15] B. Crauder, Birational morphisms of smooth algebraic threefolds collapsing three surfaces to a point, Duke Math. J. 48, 1981, 589-632.

[16] S. D. Cutkosky, Local factorization of birational maps, Advances in Math. 132, 1997, 167-315.

[17] S. D. Cutkosky, Local factorization and monomialization of morphisms, Astérisque 260. Soc. Math. France, 1999.

[18] S. D. Cutkosky, Monomialization of morphisms from 3-folds to surfaces, preprint math.AG/0010002.

[19] S. D. Cutkosky and O. Piltant, Monomial resolutions of morphisms of algebraic surfaces, preprint math.AG/9910076.

[20] V. I. Danilov, The geometry of toric varieties, Russian Math. Surveys 1978, 33:2, 97-154.

[21] V. I. Danilov, Birational geometry of toric 3-folds, 1983, 21, 269-280.

[22] A.S. Demushkin, Combinatorial invariance of toric singularities (Russian), Vestnik Moskov.Univ. Ser. I Mat.Mekh 1982, no $2,80-87$

[23] I. V. Dolgachev and Y. Hu, Variation of geometric invariant theory quotients, Inst. Hautes Études Sci. Publ. Math. 87 (1998), 5-56.

[24] G. Ewald, Blow-ups of smooth toric 3-varieties, Abh. Math. Sem. Univ. Hamburg, 57, 1987, 193-201.

[25] W. Fulton, Introduction to Toric Varieties, Annals of Mathematics Studies 131, Princeton University Press 1993.

[26] A. Grothendieck Elements de Géometrie Algébrique IV, Ibid 20 (1964).

[27] Gubeladze J.Pure Appl. Algebra 129, 35-65 (1998)

[28] R. Hartshorne, Algebraic Geometry, Graduate Texts in Mathematics 52, Springer-Verlag, 1977.

[29] H. Hironaka, On the theory of birational blowing-up, Harvard University Ph.D. Thesis 1960.

[30] H. Hironaka, An example of a non-Kählerian complex-analytic deformation of Kählerian complex structure, Annals of Math. (2), 75, 1962, 190-208.

[31] H. Hironaka, Resolution of singularities of an algebraic variety over a field of characteristic zero, Annals of Math. 79, 1964, 109-326.

[32] H. Hironaka, Flattening theorem in complex analytic geometry, Amer. J. of Math., 97, 1975, 503-547.

[33] Y. Hu, The geometry and topology of quotient varieties of torus actions, Duke Math. J. 68 (1992), 151-184; Erratum, Duke Math. J. 68 (1992), 609.

[34] Y. Hu, Relative geometric invariant theory and universal moduli spaces, Internat. J. Math. 7 (1996), 151-181.

[35] K. Karu, Boston University dissertation, 1999. http://math.bu.edu/people/kllkr/th.ps

[36] K. Kato, Toric singularities, Amer. J. Math. 116, 1994, 1073-1099.

[37] Y. Kawamata, The cone of curves of algebraic varieties, Ann. of Math. (2) 119 (1984), 603-633.

[38] Y. Kawamata, Crepant blowing-ups of three dimensional canonical singularities and its application to degenerations of surfaces, Ann. of Math. 127 (1988), 93-163.

[39] G. Kempf, F. Knudsen, D. Mumford and B. Saint-Donat, Toroidal embeddings I, 1973, 339, Lecture Notes in Mathematics, Springer.

[40] J. Kollár, The cone theorem. Note to a paper: "The cone of curves of algebraic varieties" 38] by Y. Kawamata, Ann. of Math. (2) 120 (1984), 1-5.

[41] V.S. Kulikov, Decomposition of a birational map of three-dimensional varieties outside codimension 2, Math. USSR Izvestiya 21, 1983, 187-200.

[42] D. Luna, Slices étales. Sur les groupes algébriques, p 81-105. Bull. Soc. Math. France, Paris, Mémoire 33. Soc. Math. France, Paris, 1973.

[43] K. Matsuki, Introduction to the Mori program, Springer Verlag, Berlin, to appear.

[44] K. Matsuki, Lectures on factorization of birational maps, RIMS preprint, 1999.

[45] K. Matsuki and R. Wentworth, Mumford-Thaddeus principle on the moduli space of vector bundles on an algebraic surface, Int. J. of Math.,8 (1997) 97-148.

[46] T. Oda, Torus embeddings and applications, Based on joint work with Katsuya Miyake. Tata Inst. Fund. Res., Bombay, 1978.

[47] J. Milnor, Morse Theory, Annals of Math. Stud. 51, Princeton Univ. Press, 1963.

[48] B. Moishezon, On n-dimensional compact varieties with $n$ algebraically independent meromorphic functions, Amer. Math. Soc. Trans. 63, 1967, 51-177.

[49] R. Morelli, The birational geometry of toric varieties, J. Alg. Geom. 5 1996, 751-782.

[50] R. Morelli, Correction to "The birational geometry of toric varieties" , 1997 http://www.math.utah.edu/ morelli/Math/math.html

[51] S. Mori, Threefolds whose canonical bundles are not numerically effective, Annals of Math. 1982,116, $133-176$.

[52] S. Mori, Flip theorem and the existence of minimal models for 3-folds, Journal of AMS 1, 1988, 117-253.

[53] D. Mumford, J. Fogarty and F. Kirwan, Geometric Invariant Theory (Third Enlarged Edition), Ergebnisse der Mathematik und ihrer Grenzgebiete, 34, Springer-Verlag 1992.

[54] , M. Nagata On rational surfaces I, Mem. Coll. Sci., Kyoto, A 32 (1960) 351-370.

[55] , M. Nagata Imbedding of an abstract variety in a complete variety, J.Math. Kyoto Univ. 2 (1962), 1-10

[56] T. Oda, Convex Bodies and Algebraic Geometry, Springer-Verlag, 15, 1988. 
[57] H. Pinkham, Factorization of birational maps in dimension 3, Proceedings of Symposia in Pure Math. $40,1983$.

[58] M. Reid, Canonical threefolds, Géométrie Algébrique Angers 1979, A. Beauville ed., Sijthoff and Nordhoff 1980, 273-310.

[59] M. Reid, Minimal models of canonical 3-folds, Adv. Stud. in Pure Math. 1, 1983, 131-180.

[60] M. Reid, Decomposition of Toric Morphisms, Arithmetic and Geometry, papers dedicated to I. R. Shafarevich on the occasion of his 60th birthday, vol. II, Progress in Math. (M. Artin and J. Tate eds.) 36, 1983, 395-418.

[61] M. Reid, Birational geometry of 3-folds according to Sarkisov, preprint 1991

[62] J. Sally, Regular overrings of regular local rings, Trans. Amer. math. Soc. 171, 1972, 291-300.

[63] V. G. Sarkisov, Birational maps of standard $\mathbb{Q}$-Fano fiberings, I. V. Kurchatov Institute Atomic Energy preprint, 1989.

[64] M. Schaps, Birational morphisms of smooth threefolds collapsing three surfaces to a curve, Duke Math. J. 48, 1981, 401-420.

[65] D. L. Shannon, Monoidal transforms, Amer. J. Math. 1973, 45, 284-320.

[66] V. V. Shokurov, A nonvanishing theorem, Izv. Akad. Nauk SSSR Ser. Mat. 49 (1985), 635-651.

[67] H. Sumihiro, Equivariant Completion I, II, J. Math. Kyoto Univ. 14, 15, 1974, 1975, 1-28, 573-605.

[68] M. Teicher, Factorization of a birational morphism between 4-folds. Math. Ann. 256 (1981), 391-399.

[69] M. Thaddeus, Stable pairs, linear systems and the Verlinde formula, Invent. Math. 117, 1994, 317-353.

[70] M. Thaddeus, Geometric invariant theory and flips, J. Amer. Math. Soc. 9, 1996, 691-723.

[71] O. Villamayor, Constructiveness of Hironaka's resolution. Ann. Sci. École Norm. Su (4) 22 (1989), no. 1, 1-32.

[72] J. Włodarczyk, Decomposition of birational toric maps in blow-ups and blow-downs. A proof of the Weak Oda Conjecture, Transactions of the AMS 349, 1997, 373-411.

[73] J. Włodarczyk, Birational cobordisms and factorization of birational maps, J. Alg. Geom. 9 (2000), 425-449

[74] O. Zariski, Algebraic Surfaces, Springer-Verlag, 1934.

Department of Mathematics, Purdue University, West Lafayette, IN-47907, USA

E-mail address: wlodar@math.purdue.edu, jwlodar@mimuw.edu.pl 